\title{
8 Bewertungsinteraktionen in Pausengesprächen im Theater
}

Kapitel 3 dieser Arbeit zeigte, dass das Bewerten bei der kommunikativen Aneignung von Fernsehinhalten und Kunstwerken eine zentrale kommunikative Aufgabe in Gesprächen zwischen den Rezipienten ist. Auch die dieser Arbeit zugrunde liegenden Gespräche unter Theaterzuschauern bestätigen, dass Bewertungen in der Kommunikation im Anschluss an die Rezeption eines Theaterstücks in hohem Maße von den am Gespräch Beteiligten relevant gesetzt werden (vgl. Hrncal/Gerwinski 2015 sowie Linz/Hrncal/Schlinkmann 2016). Im Fokus der nachfolgenden Analyse von Bewertungsinteraktionen in Pausengesprächen im Theater(foyer) steht deshalb die Frage nach charakteristischen Ausprägungen der interaktiven Verfertigung von Bewertungen. Da die Bewertungsinteraktionen in den Gesprächen eingebettet in das gesellige Ereignis des Theaterbesuchs realisiert werden (siehe Kapitel 4), schließt sich für die folgenden Kapitel zudem die Frage an, inwiefern der gesellige Kontext von den Beteiligten selbst relevant gesetzt wird und inwiefern er eine unterstützende Ressource für den Umgang der mit Bewertungen im Kontext der Kunstkommunikation verbundenen Zugzwänge und einer ebenfalls damit verbundenen potenziellen Face-Bedrohung darstellt.

Die Struktur dieses Kapitels bildet die sequenzielle Struktur der Bewertungsinteraktionen in den dieser Arbeit zugrunde liegenden Gesprächen ab. In einem ersten Schritt der Analyse erfolgt ein Blick auf Möglichkeiten der Initiierung von Bewertungsinteraktionen sowie auf ihren sequenziellen und thematischen Verlauf. Bei der Betrachtung von Formen und der Aushandlung von Nichtübereinstimmung liegt der Fokus vor allem auf den von den Beteiligten genutzten Verfahren, um Nichtübereinstimmung zu bearbeiten. Im Anschluss daran werden Möglichkeiten des Ausstiegs aus Bewertungsinteraktionen in den vorliegenden Gesprächen aufgezeigt.

\subsection{Initiierung von Bewertungsinteraktionen}

Die Initiierung einer Bewertungsinteraktion kann in den dieser Arbeit zugrunde liegenden Daten zu verschiedenen Zeitpunkten im Gespräch erfolgen: Sie kann zum einen mit dem Gesprächsbeginn zusammenfallen, unmittelbar nachdem die Aufnahmegeräte eingeschaltet wurden, ohne dass die Aufnahmesituation thematisiert wird oder im Anschluss an einen Verweis auf die Aufnahmesituation beziehungsweise die Aufnahmegeräte (siehe Kapitel 6.3; vgl. auch Hrncal 
2018 sowie Gerwinski/Linz 2018). Zum anderen kann sie an eine Gesprächsphase anschließen, in der die Beteiligten das weitere Vorgehen abstimmen, in der phatische Kommunikation beziehungsweise Small Talk betrieben wird oder in der situationsbezogene Aspekte thematisiert werden (unter anderem das Verständigen über den Ort, an dem das Gespräch weitergeführt werden soll, das Identifizieren von Schauspielern, oder das Verweisen auf räumliche Gegebenheiten der Spielstätte). Unabhängig vom Zeitpunkt der Initiierung finden sich in den dieser Arbeit zugrunde liegenden Gesprächen folgende Formen des Einstiegs in eine Bewertungsinteraktion:

1. Die am Gespräch Beteiligten fordern mittels unterschiedlicher Gradationsstufen der Konkretisierung ihr Gegenüber durch eine Frage explizit zu einer (bewertenden) Stellungnahme auf. Der Grad der Konkretisierung kann von der offenen Ein-Wort-Frage „und?“ (siehe Beispiel 62, Kapitel 8.1.1) über eine näher spezifizierte Frage wie „und? wie findes_du_s“ (siehe Beispiel 61, Kapitel 8.1.1) bis hin zu einer näher spezifizierten Frage mit der Nennung eines konkreten Bezugsobjekts wie „wie FINdest du die muSIK“ (siehe Beispiel 67, Kapitel 8.1.1), unter anderem auch begleitet von einer adressatenspezifischen Ergänzung wie „und was SAGST du; als JUgendliche“ (siehe Beispiel 63, Kapitel 8.1.1) reichen.

2. Die am Gespräch Beteiligten fordern von ihrem Gegenüber mittels einer Bewertungsunterstellung mit angeschlossenem Rückversicherungssignal (vgl. Schwitalla 2002) explizit eine (bewertende) Stellungnahme ein (zum Beispiel „!DU! findest es ja GANZ schlimm.=ne?“, siehe Beispiel 70, Kapitel 8.1.1).

3. Die am Gespräch Beteiligten geben eine selbstinitiierte Bewertung ab, ohne explizit eine Zweitbewertung einzufordern (zum Beispiel „oh VOLL gu:tdie inszeNIErung“, siehe Beispiel 72, Kapitel 8.1.3).

4. Die am Gespräch Beteiligten geben eine selbstinitiierte Bewertung ab und fordern explizit eine (bewertende) Stellungnahme ihres Gegenübers ein (zum Beispiel „aber TOLle schauspieler- find_ste nich?“, siehe Beispiel 74, Kapitel 8.1.4).

5. Die am Gespräch Beteiligten geben eine selbstinitiierte Bewertung ab und schreiben der vorangegangenen Äußerung ihres Gegenübers den Status einer Bewertung zu (zum Beispiel „ja ich finds auch COOL dass_es_so::. (0.6) mit MUsical:. (0.4) QUA:si (.) elementen ist.“, siehe Beispiel 75, Kapitel 8.1.5).

Diese fünf Formen der Initiierung von Bewertungsinteraktionen werden in den folgenden Unterkapiteln differenzierter und anhand exemplarischer Ausschnitte beschrieben. 


\subsection{1 (Bewertungs-)Einforderungen durch Fragen}

In den beiden nachfolgend angeführten Ausschnitten steigen die Beteiligten durch eine explizite Einforderung einer (bewertenden) Stellungnahme von ihrem Gegenüber mittels der Ein-Wort-Frage „und?“ in die Bewertungsinteraktion ein. Der erste Ausschnitt ${ }^{84}$ entstammt einem Gespräch zwischen dem Ehepaar ${ }^{85}$ Ina und Finn in der Pause des Theaterstücks Der Streik (siehe Kapitel 6.6), in dem es um die wirtschaftliche Entwicklung der USA in den 1950er Jahren geht. Ina und Finn verständigen sich auf dem Weg vom Theatersaal ins Theaterfoyer darüber, ob Finns Aufnahmegerät läuft und beschließen, Getränke zu organisieren. Ina initiiert unmittelbar daran anschließend mit ihrer zunächst nicht näher spezifizierten (Ein-Wort-)Frage „und?“ (Z. 069) die Bewertungsinteraktion (siehe auch Beispiel 26, Kapitel 7.3):

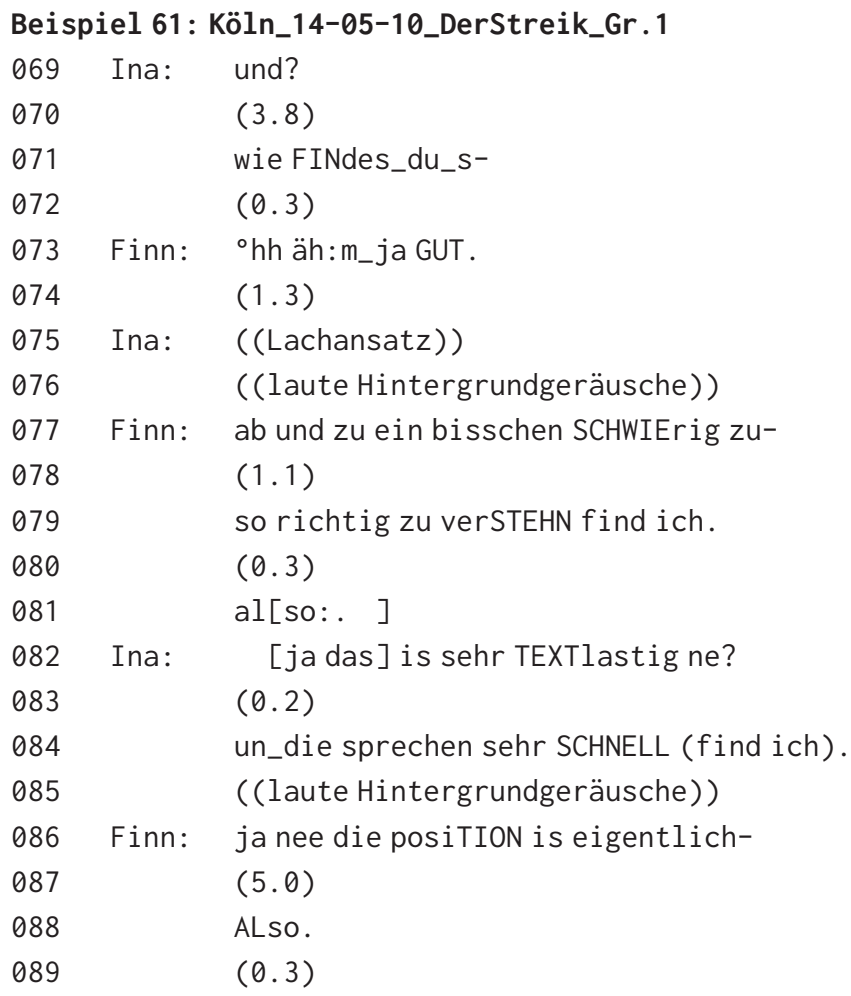

84 Siehe zu einer früheren Analyse dieses Beispiels Hrncal (2018: 247-248). 85 Genauere Informationen zu den jeweiligen Konstellationen der Gesprächsgruppen können Kapitel 6.7 entnommen werden. 
090

091

092

093

094

095
WER jetz hier (.) wElche position?

(1.6)

(da sin)

((laute Hintergrundgeräusche))

(0.5)

da 1 [iegen] die SCHUhe übrigens?

Die durch Ina von Finn eingeforderte Stellungnahme (Z. 069) bleibt vorerst aus. Mit ihrer Nachfrage „wie FINdes_du_s-“ (Z. 071) fordert Ina daraufhin - ohne eine Konkretisierung ihres Verweises auf das Bezugsobjekt mittels der Proform „es“ vorzunehmen - explizit eine (Global-)Bewertung von Finn ein, die dieser in Zeile 073 mit einem Einatmen, dem Verzögerungssignal „ähm“ und der Gesprächspartikel „ja“ (vgl. Marić 2017) als Vorlaufelemente hinauszögert. Mit dem evaluierenden Adjektiv „GUT“ (Z. 073) realisiert Finn dann eine positive Bewertung und nimmt so eine erste Positionierung hinsichtlich des Bezugsobjekts („es“) vor, allerdings ohne die Bewertung in irgendeiner Weise zu spezifizieren oder zu begründen. Nach einem Lachen oder Husten Inas ${ }^{86}$ (Z. 075) schränkt Finn seine positive Bewertung mit einer durch Pausen verzögerten und abgeschwächten Äußerung vorsichtig ein (,ab und zu ein bisschen SCHWIErig zu- (1.1) so richtig zu verSTEHN find ich. (0.3) also:.“, Z. 077-081), die er zudem durch die nachgestellte explizit subjektive Perspektivierung mit Verbspitzenstellung („find ich“, Z. 079) in ihrem Geltungsanspruch abmildert. Die mit dem Verzögern und Abschwächen seiner Bewertung von Finn getroffenen Vorsichtsmaßnahmen projizieren bereits eine potenziell nicht übereinstimmende Zweitbewertung Inas sowie eine potenziell damit für Finn verbundene Gesichtsbedrohung (vgl. Auer/Uhmann 1982). In Zeile 081 scheint Finn mit weiteren Erläuterungen fortfahren zu wollen, wird aber durch Inas Äußerung (Z. 082) unterbrochen. Ina nimmt ab Zeile 082 eine Konkretisierung des von Finn geäußerten vagen Verweises auf Verständnisschwierigkeiten (Z. 077-081) durch den Verweis auf die Textlastigkeit (Z. 082) und das schnelle Sprechen der Schauspieler im Stück (Z. 084) vor. Sowohl die prädikative Konstruktion, die Gradpartikel „sehr“ als auch das eine unzweckmäßig hohe Ausprägung indizierende und damit negativ konnotierte Suffix „-lastig“ weisen darauf hin, dass es sich bei Inas Äußerung „ja das is sehr TEXTlastig ne?“ (Z. 082) um eine Zweitbewertung handelt und dass sie Finns Äußerungen (Z. 077-081) den Status einer negativen Bewertung zuschreibt. Durch das ihrer Bewertung angeschlossene Rückversicherungssignal „ne“ (Z. 082) fordert sie von Finn eine Bestätigung ihrer Bewertung

86 Der Audioaufnahme ist nicht eindeutig zu entnehmen, ob es sich um einen Lachansatz oder um ein Husten handelt. 
ein. Diese Bestätigung liefert Finn allerdings nicht, sondern widerspricht Ina (Z. 086) und weist sehr vage darauf hin, dass sich seine Verständnisschwierigkeiten auf die im Stück durch die Schauspieler eingenommenen Positionen beziehen und nicht den Textanteil oder die Sprechgeschwindigkeit der Schauspieler (Z. 086). Das durch Pausen verzögerte Hervorbringen der teilweise unvollständigen Äußerungen Finns ist zudem ein Indiz dafür, dass es Finn selbst schwerfällt, seine zuvor angesprochenen Verständnisschwierigkeiten (Z. 077-079) zu präzisieren. Der für Ina möglicherweise gesichtsbedrohende Widerspruch Finns (,ja nee“, Z. 086) sowie die Vagheit seiner Äußerungen werden von den beiden nicht weiter kommunikativ bearbeitet. Aufgrund von lauten Hintergrundgeräuschen und einem lokal sensitiven Verweis (vgl. Bergmann 1988) auf Objekte im gemeinsamen Wahrnehmungsraum (Z. 092-095) wird die Bewertungsaushandlung vorerst unterbrochen. Dieser erste kurze gemeinsame Austausch dient Ina und Finn, eingeleitet durch eine eingeforderte Bewertung und deren Aushandlung scheinbar auch dazu, gegebenenfalls durch Schwierigkeiten bei der (akustischen) Wahrnehmung während der Rezeption entstandene (Wissens-)Lücken hinsichtlich des Theaterstücks auszuhandeln beziehungsweise auszuräumen (vgl. Klemm 2001: 88 sowie Kapitel 3.1)

Auch im nächsten Ausschnitt ${ }^{87}$ aus dem Gespräch zwischen Titus und Vanessa im Anschluss an das Theaterstück Die Lücke (siehe Kapitel 6.6), in dem es um den Nagelbombenanschlag der rechtsextremen terroristischen Vereinigung „Nationalsozialistischer Untergrund“ (NSU) in der Kölner Keupstraße im Jahr 2004 geht, wird die erste Bewertungssequenz mit der nicht näher spezifizierten (Ein-Wort-)Frage „und?“ (Z. 005) initiiert (siehe auch Beispiel 28, Kapitel 7.3). Zu Beginn des Gesprächs vergewissern sich die beiden Beteiligten, dass das Aufnahmegerät tatsächlich eingeschaltet ist. Der Einstieg in die erste Bewertungssequenz schließt sich an eine minimale Vorlaufphase an, in der die Aufnahmesituation durch Titus' Verweis auf das Funktionieren der Aufnahme zum Thema gemacht wird (siehe Kapitel 6.3). Die von Vanessa durch „und?“ (Z. 005) relevant gesetzte Stellungnahme wird von Titus in Form einer Bewertung (Z. 007) gefolgt von einem Lachen geliefert, ohne dass Vanessa nochmals nachfragen muss:

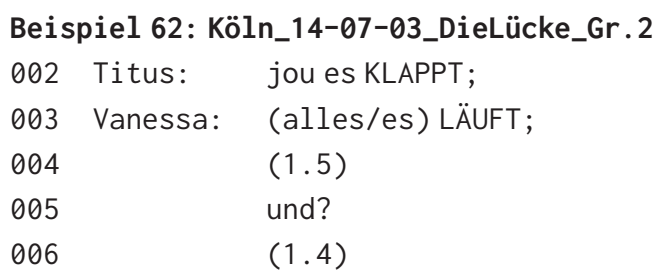

87 Siehe zu einer früheren Analyse dieses Beispiels Hrncal (2018: 248-249). 
007 Titus: ja es [war schon KRASS also hehehe]

008 Vanessa: [(sagste) als äh am SCHLUSS, ]

Titus' Erstbewertung erfolgt nicht in unmittelbarem Anschluss an Vanessas Frage, sondern wird von ihm durch eine Pause verzögert (Z. 006). Das Bezugsobjekt seiner Bewertung hält Titus - wie auch Finn im vorherigen Beispiel mit der Proform „es“ vage, da nicht deutlich wird, ob er sich auf die gesamte Inszenierung oder einen (Teil-)Aspekt des Theaterstücks bezieht. Auch das nicht eindeutig positiv oder negativ konnotierte Adjektiv „KRASS“88 trägt zur Vagheit seiner Äußerung bei, die im weiteren Verlauf des Gesprächs zwischen den beiden Gesprächspartnern nicht aufgelöst wird. An dieser Stelle nutzt Titus möglicherweise die Uneindeutigkeit des Adjektivs, um so eine eindeutige Positionierung hinsichtlich des Bezugsobjekts zu umgehen. In Überlappung mit Titus' Bewertung spezifiziert Vanessa, trotz ihrer in Zeile 005 vorgenommenen Einforderung einer Stellungnahme von Titus, ihre Aufforderung mit Fokus auf das Ende des Theaterstücks (Z. 007). Titus kommt jedoch nicht dazu, zu Vanessas erneuter Frage Stellung zu nehmen, da die Interaktion der beiden von einer anderen im Foyer anwesenden Person unterbrochen wird. ${ }^{89}$

Im folgenden Ausschnitt ${ }^{90}$ wird die Bewertungsinteraktion durch eine im Vergleich zu den beiden vorangehenden Ausschnitten komplexere, mit „und“ eingeleitete und mit einer Rollenzuschreibung verbundene Frage initiiert. Der Ausschnitt entstammt einem Gespräch zwischen Dagmar, Bianca, Lydia und Karla in der Pause des Theaterstücks Der gute Mensch von Sezuan. Nach der gegenseitigen Absicherung, dass ihre Aufnahmegeräte laufen, und nach einer längeren Gesprächspause im Anschluss an das Verlassen des Theatersaals steigt Dagmar in die Bewertungsinteraktion ein:

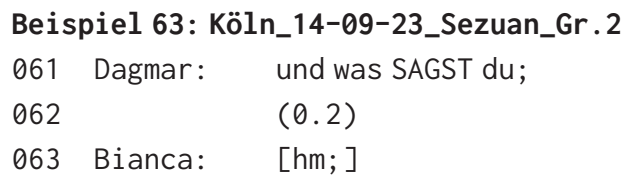

88 Vergleiche zur Uneindeutigkeit von „KRASS“ auch den Duden-Eintrag (Bedeutungsübersicht) zu ,krass': 1. in seiner Art besonders extrem; 2.a. (besonders Jugendsprache) in begeisternder Weise gut, schön; 2.b. (besonders Jugendsprache) schlecht, furchtbar. (http://www. duden.de/rechtschreibung/krass, 20.02.2018).

89 Die unbekannte Person fragt Titus, ob er seine Karte noch benötigt. Hintergrund dieser Frage ist, dass das Theaterticket im Anschluss an die Veranstaltung als Fahrkarte für den öffentlichen Personennahverkehr genutzt werden kann.

90 Vergleiche auch Habscheid (2018: 189-194) zu einer Diskussion dieses Ausschnitts mit Bezug zu den Aspekten Konversation, Small Talk und Bildungssprache. 


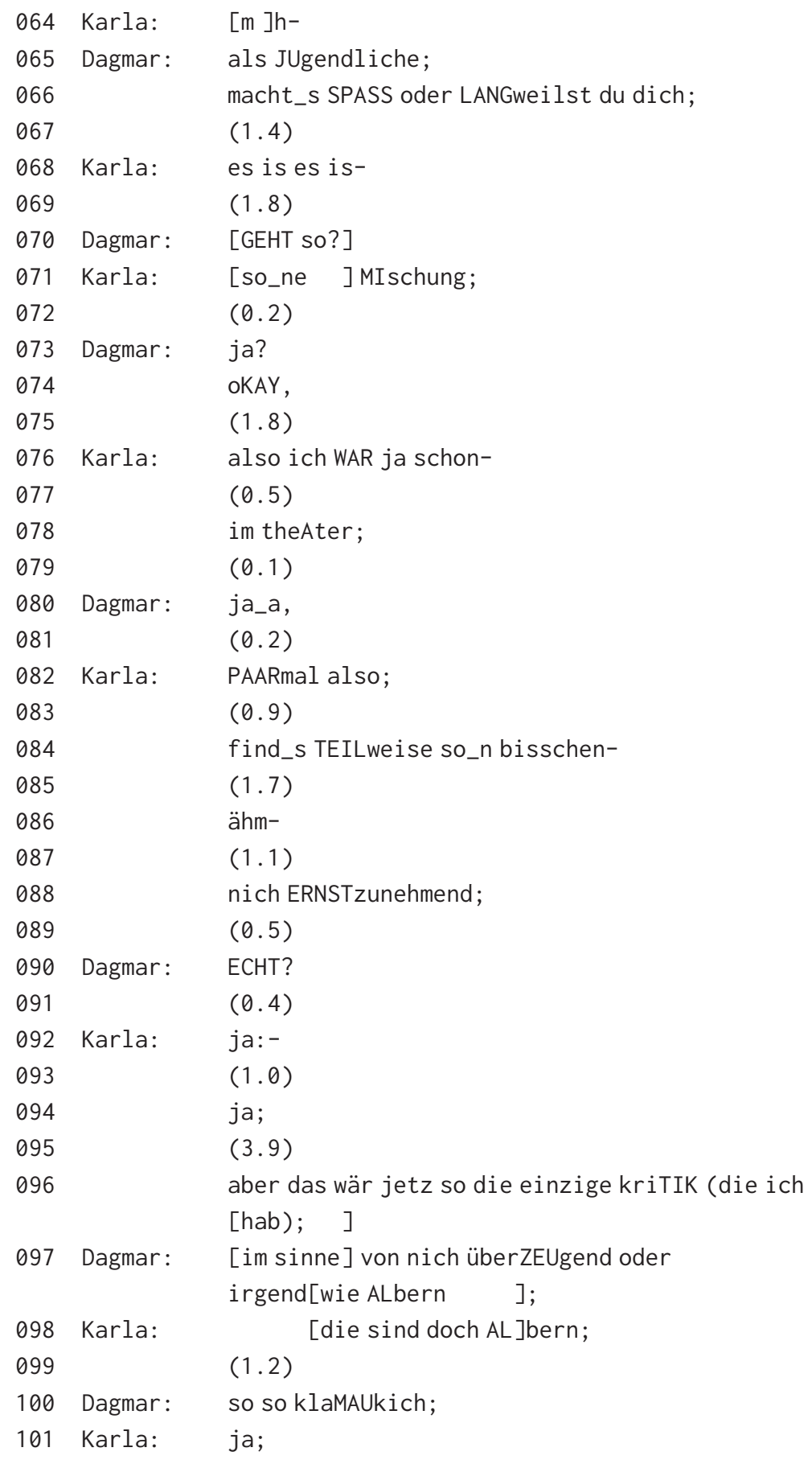




$\begin{array}{lll}102 & \text { Dagmar: } & \text { mit den PUPpen und dem gAn[zen; ] } \\ 103 & \text { Karla: } & \\ 104 & & (0.3) \\ 105 & \text { Dagmar: } & \text { oKAY? } \\ 106 & & (1.6)\end{array}$

Ihre Einforderung einer Stellungnahme (Z. 061) richtet Dagmar an Lydias Tochter Karla. Nach einer kurzen Pause und nach einem Zögern Karlas (Z. 064) adressiert Dagmar sie als Jugendliche und Vertreterin ihrer Altersgruppe (Z. 065), stellt zwei Antwortmöglichkeiten zur Auswahl (,macht_s SPASS oder LANGweilst du dich;“, Z. 066) und setzt damit für Karla den Entertainment-Charakter des Theaterstücks als für die Bewertung ausschlaggebenden Maßstab relevant. Karla setzt in Zeile 068 zögerlich zu einer Antwort an, die Dagmar mit der fragend intonierten Bewertungsunterstellung „GEHT so?“ (Z. 070) vorwegnimmt. In Überlappung liefert Karla eine vorerst noch sehr vage Stellungnahme (Z. 071), die sie im Anschluss sukzessive konkretisiert. Dabei verweist sie zunächst auf bisherige Theaterbesuche (Z. 076-082) und äußert erst dann eine durch Pausen verzögerte subjektive Stellungnahme, die sie zudem durch Heckenausdrücke abschwächt („find_s TEILweise so_n bisschen- (1.7) ähm- (1.1) nich ERNSTzunehmend;“, Z. 084-088) und so als potentiell heikel markiert. Bei ihrer Stellungnahme greift Karla nicht auf eine der von Dagmar vorgegebenen Antwortmöglichkeiten (Spaß vs. Langeweile) zurück, sondern nimmt das Theaterstück auf der globalen Ebene in den Fokus. Dagmars betonte und in der Intonation stark steigende Nachfrage (Z. 090) weist bereits darauf hin, dass sie Karlas Meinung bezüglich des Theaterstücks nicht teilt, und fordert gleichzeitig eine Bestätigung ein, die Karla in den Zeilen 092 und 094 auch liefert. Nach einer längeren Pause merkt Karla in Zeile 096 an, dass dies die „einzige kriTIK“ an dem Theaterstück sei und schreibt mit dieser Äußerung ihrer Stellungnahme (Z. 084-088) zudem nachträglich den Status einer negativen Bewertung zu. Dagmar fordert darauf folgend weitere Konkretisierungen ein (Z. 097) und bietet Karla nochmals zwei Auswahlmöglichkeiten an (,im sinne von nich überZEUgend oder irgendwie ALbern;“), während Karla in Überlappung bereits auf die Inszenierung vermutlich im Stück agierender Figuren verweist (,die sind doch ALbern;“, Z. 098), das Bezugsobjekt ihrer Äußerung allerdings nicht weiter konkretisiert. Dagmar bietet einen weiteren Formulierungsvorschlag an (Z. 100) und schließt an Karlas Bestätigung (Z. 101) mit einer Konkretisierung („mit den PUPpen und dem gAnzen;“, Z. 102) an, die Karla ebenfalls bestätigt. Dagmar quittiert dies mit einem steigend intonierten „oKAY?“ (Z. 103). Dieses Beispiel zeigt, wie von den Beteiligten bei der Initiierung von Bewertungsinteraktionen mithilfe einer adressatenorientiert zugeschnittenen Nachfrage eine spezi- 
fische Perspektive (in diesem Falle die der Jugendlichen) relevant gesetzt wird, und wie Dagmar ihrer jugendlichen Gesprächspartnerin Karla Impulse liefert, um sie zur Konkretisierung ihrer eingangs vage formulierten Stellungnahme $\mathrm{zu}$ bewegen und sie damit möglicherweise auch an die Praxis der Kunstkommunikation heranführt.

Neben der Initiierung der Bewertungsinteraktion durch die Ein-Wort-Frage „und?“ greifen die Sprecher auch auf Formulierungen zurück, die explizit eine Bewertung erfragen. Dies ist im folgenden Gespräch zwischen Anna, Pascal, Annalena und Jasmin im Anschluss an das Theaterstück Verrücktes Blut (siehe Kapitel 6.6), das Migration, Bildung und Integration thematisiert, der Fall. Der Gesprächsausschnitt ${ }^{91}$ beginnt, nachdem die Beteiligten sich darüber verständigt haben, den Theatersaal zu verlassen und dass die Aufnahmegeräte das Gespräch aufzeichnen. In Zeile 027 wird die Bewertungsinteraktion von Anna durch die geschlossene Frage „hat_s euch geFALlen?“ initiiert, mit der sie die anderen Gruppenmitglieder explizit zu einer Bewertung auffordert:

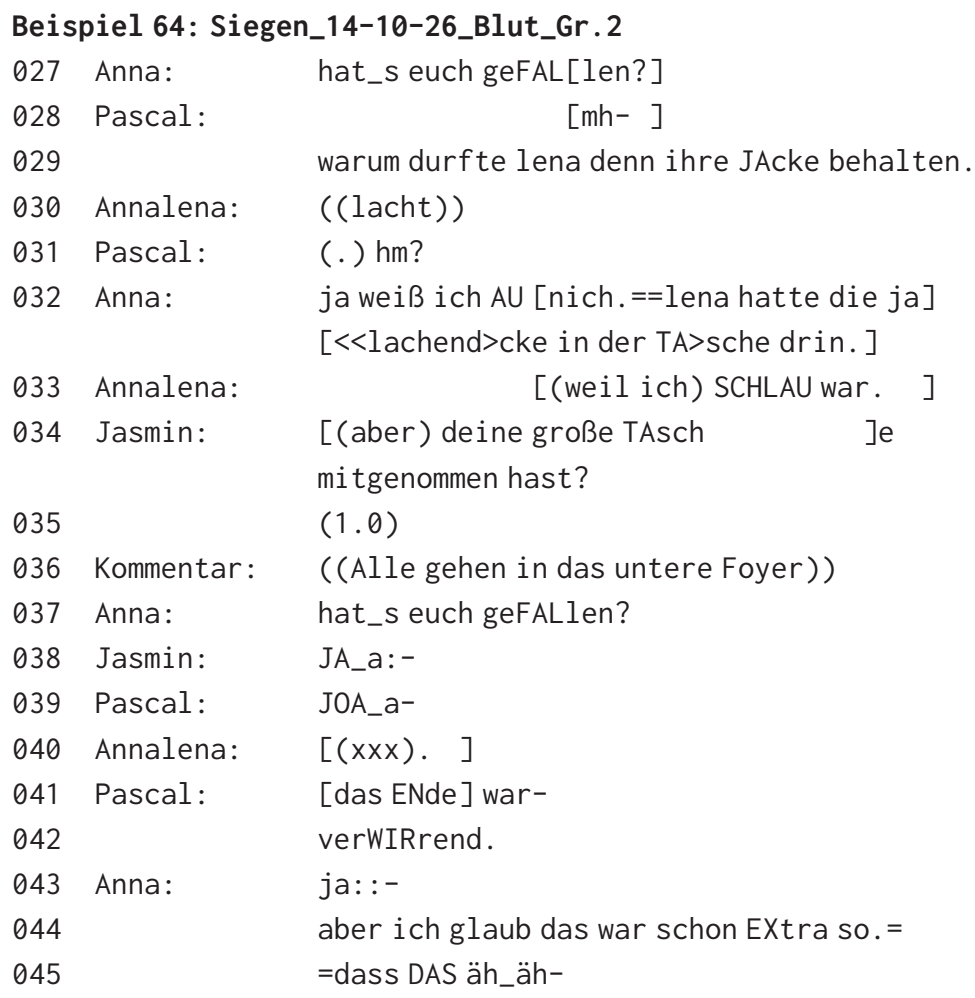

91 Siehe zu einer früheren Analyse dieses Beispiels Hrncal (2018: 249-251). 


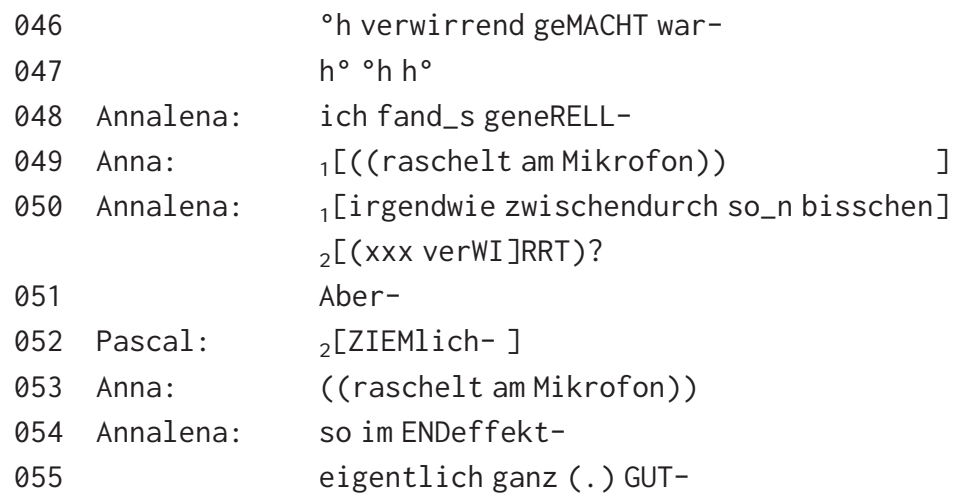

Pascal reagiert auf Annas Frage beziehungsweise Bewertungseinforderung mit einem Zögern (Z. 028) und initiiert durch die Frage, warum „lena denn ihre JAcke behalten“ (Z. 029) durfte, einen Themenwechsel. In den folgenden Turns (Z. 032-034) machen die Beteiligten weiterhin Lenas Tasche und die sich darin befindende Jacke zum Fokus ihrer Interaktion. Als die Gruppe das Theaterfoyer erreicht hat, wiederholt Anna in Zeile 037 ihre bereits zu Beginn des Ausschnitts gestellte Frage „hat_s euch geFALlen?“ und markiert so, dass die von ihr zu Beginn des Ausschnitts erfragte Bewertung von den anderen Beteiligten noch nicht in einem ausreichenden Maße geliefert wurde (vgl. Goodwin/Goodwin 1987: 17). Im Gegensatz zu Annas erstem Initiierungsversuch liefern Pascal, Annalena und Jasmin die durch Annas erneute Nachfrage relevant gesetzte Stellungnahme in Form von minimaler Zustimmung (,JA_a:“, Z. 038 beziehungsweise „JOA_a“, Z. 039), an die sich Pascals Stellungnahme zum Ende des Theaterstücks anschließt (Z. 041-042). Anna reagiert auf Pascals Stellungnahme mit partiellem Widerspruch (,ja::- aber“ Z. 043-044), verweist auf die von ihr vermutete hinter der Inszenierung stehende Absicht der Beteiligten (Z. 044-046), und schreibt damit Pascals Stellungnahme den Status einer negativen Bewertung zu (Z. 044). In Zeile 048 setzt Annalena zu einer allgemeinen Bewertung an (,ich fand_s geneRELL“), fährt dann aber mit ihrer mehrfach durch Heckenausdrücke abgeschwächten Äußerung ,irgendwie zwischendurch so_n bisschen“ (Z. 050) fort, wobei sie Pascals Prädikation (Z. 041-042) aufgreift und vermutlich zur Beschreibung ihres eigenen Zustands nutzt (,xxx verWIRRT“, Z. 050). Ab Zeile 051 kommt Annalena, eingeleitet durch den Widerspruchsmarker „Aber“, mit dem sie sich von ihrer eigenen vorherigen Äußerung zu distanzieren scheint, schließlich zu dem allgemeineren und positiven Urteil, dass das Theaterstück „so im ENDeffekt- eigentlich ganz (.) GUT-“ (Z. 054-055) war. Indem Annalena ihre Globalbewertung durch den Heckenausdruck „eigentlich“ und die Gradpartikel „ganz“ abschwächt, markiert sie 
ihre positive Globalbewertung und die damit verbundene Positionierung als potentiell heikel und Face-bedrohend (vgl. König 2014).

Auch im folgenden Gesprächsauschnitt ${ }^{92}$ wird die Bewertungsinteraktion durch eine explizite Erfragung einer bewertenden Stellungnahme initiiert. Elfriede, Imke und Mandy unterhalten sich in der Pause des Theaterstücks Alle sieben Wellen (siehe Kapitel 6.6), das von der E-Mail-Beziehung eines Paares handelt. In diesem Beispiel stellt Elfriedes scherzhafte Nachfrage „na (.) HAT_S euch vom sit- (0.4) HOcker gehaun?“ (Z. 002-004) den Einstieg in die erste Bewertungssequenz und gleichzeitig auch den Beginn des Gesprächs dar:

$\begin{array}{ll}\text { Beispiel 65: Siegen_15-01-14_7Wellen_Gr.2 } \\ 001 \text { Elfriede: } & \text { Okay. } \\ 002 & \text { na (.) HAT_S euch vom sit- } \\ 003 & (0.4) \\ 004 & \text { HOcker gehaun? } \\ 005 & (0.3) \\ 006 & \text { ne? } \\ 007 & (1.0) \\ 008 & \text { hmhm? } \\ 009 & (0.4) \\ 010 & \text { ja? } \\ 011 & (0.3) \\ 012 \text { Mandy: } & \text { HA ! } 0: \text { AH! . } \\ 013 \text { Elfriede: } & (.) \text { ha ha }{ }^{\circ}(.) \text { war schon- } \\ 014 & (0.2) \\ 015 & \text { interesSANT=ne? }\end{array}$

Elfriedes Nachfrage lässt aufgrund ihrer prosodischen Realisierung nicht nur Ironie vermuten, sondern auch darauf schließen, dass bereits eine Bewertung mitschwingt. Auffällig an diesem Einstieg in die Bewertungsinteraktion ist, dass Elfriede, durch kurze Pausen verzögert, ihrer geschlossenen Nachfrage Rückversicherungssignale in unterschiedlichen Varianten („ne?“, „hmhm?“ und „ja?“, Z. 006-010) anschließt und so mehrfach hintereinander eine Reaktion ihrer Gesprächspartnerinnen einfordert, die Mandy schließlich in Form der affektiven Vokalisation (vgl. Hartung 2000: 124, in Anlehnung an Scherer 1977: 203) „HA !O: $\mathrm{AH!“} \mathrm{(Z.} \mathrm{012)} \mathrm{liefert.} \mathrm{Wie} \mathrm{auch} \mathrm{schon} \mathrm{bei} \mathrm{ihrer} \mathrm{in} \mathrm{Zeile} 002$ gestellten Frage bleibt Elfriedes von Lachen begleitete und durch eine kurze Pause verzögerte Bewertung „(.) ha ha $h^{\circ}$ (.) war schon (0.2) interesSANT“ (Z. 013-015) hinsichtlich des

92 Siehe zu einer früheren Analyse dieses Beispiels Hrncal (2018: 251-252). 
Bezugsobjekts offen: Während sie in Zeile 002 noch mit der Proform „es“ vage auf das Bezugsobjekt referiert, wird der Gegenstand ihrer Bewertung in den Zeilen 013 bis 015 nicht (mehr beziehungsweise nochmal) verbalisiert. Zudem ist bei dem von Elfriede geäußerten evaluativen Ausdruck ,interesSANT“ (Z. 015) auf Basis der Audioaufnahme nicht eindeutig nachvollziehbar, ob es sich um eine positive oder negative Bewertung handelt. Vermutlich nutzt Elfriede hier, ähnlich wie Titus in Beispiel 62, die Uneindeutigkeit des Bewertungsausdrucks, um sich einer eindeutigen Positionierung hinsichtlich des Bewertungsobjekts zu entziehen. Die in ihrer Stellungnahme enthaltene Partikel „schon“ weist möglicherweise darauf hin, dass Elfriede die in ihrer Nachfrage (Z. 002-004) implizierte Bewertung relativiert und sich der Meinung der anderen Beteiligten anschließt. Mittels des Rückversicherungssignals „ne“ (Z. 013) fordert sie von den anderen Beteiligten eine Bestätigung ihrer Stellungnahme ein, die allerdings ausbleibt. Stattdessen schließt Imke mit der Feststellung, dass ihr Aufnahmegerät noch nicht läuft, an Elfriedes Bestätigungseinforderung an. Elfriede und Mandy erklären Imke daraufhin, wie das Aufnahmegerät eingeschaltet wird.

Die vorangehend angeführten Ausschnitte haben gezeigt, dass der Einstieg in eine Bewertungsinteraktion durch mehr oder weniger spezifizierte Fragen erfolgen kann. Bei näherer Betrachtung der Ausschnitte fällt auf, dass die Fragenden mit der Proform „es“ auf den Bewertungsgegenstand referieren, ohne dass dieser zuvor konkret als Thema eingeführt wurde. Diese Beobachtung weicht von dem von Imo (2012: 233) auf Basis privater Alltagsgespräche herausgestellten Ergebnis ab, dass der Grund für „eine strukturelle Präferenz für eine Pro-Form“ darin besteht, „dass Bewertungen meist erst erfolgen, nachdem ein Thema eingeführt ist“ (siehe auch Kapitel 2.1.2). Der Bewertungsgegenstand wird mittels des Verweises durch die Proform „es“ recht allgemein und vage gehalten, und es wird den Antwortenden somit überlassen, das Bezugsobjekt gegebenenfalls zu konkretisieren. Die zu einer Stellungnahme aufgeforderten Beteiligten erfragen allerdings in keinem der vorangehend angeführten Beispiele eine Konkretisierung des Bezugsobjekts vom Sprecher, sondern behalten entweder den vagen Modus bei oder nehmen einen bestimmten Aspekt des Theaterstücks in den Blick und so selbst eine Konkretisierung des Bezugsobjekts vor.

Die folgenden Ausschnitte zeigen eine weitere, von den Gesprächsteilnehmern genutzte Möglichkeit der Initiierung einer Bewertungsinteraktion, nämlich das Erfragen einer Bewertung im Hinblick auf einen konkreten Aspekt des Theaterstücks oder die explizite Einforderung einer Globalbewertung der Inszenierung. Die ersten beiden Ausschnitte entstammen dem Gespräch zwischen Gabriele und Cecilia in der Pause des Theaterstücks Der gute Mensch von Sezuan. Nachdem Gabriele mit Cecilia über vermutlich berufliche Belange gesprochen und eine SMS zu Ende verfasst hat, fordert sie in Beispiel 66 Cecilia durch 
ihre Frage „wer spielt am BESten was findest DU?“ (Z. 416) explizit zu einer Bewertung der schauspielerischen Leistung der Darsteller beziehungsweise eines Darstellers auf:

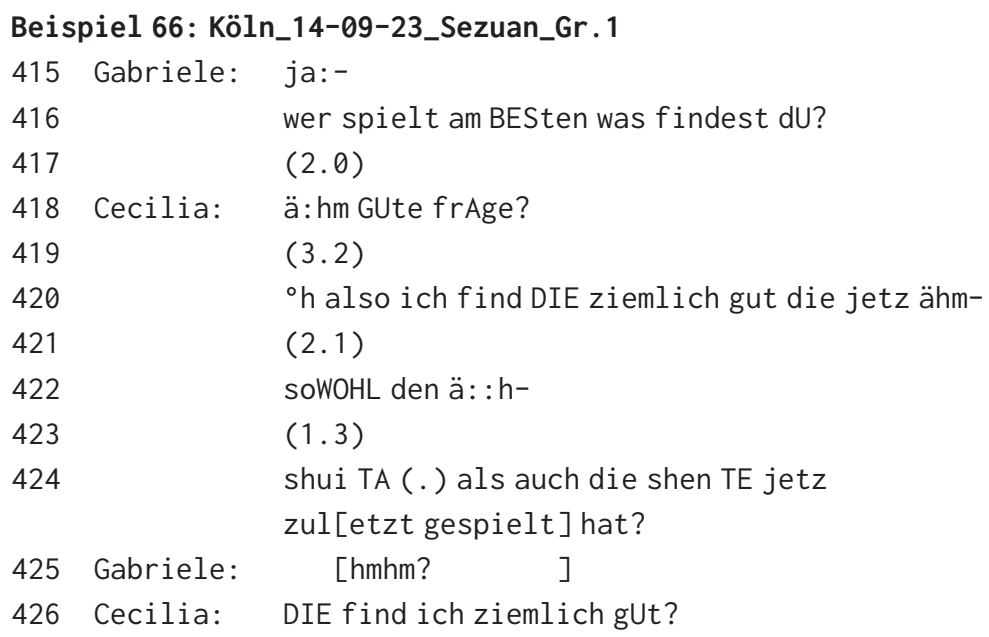

Auf Gabrieles Frage hin verzögert Cecilia mit einer kurzen Pause, dem Verzögerungssignal „ä:hm“, dem Meta-Kommentar „GUte frAge?“ (Z. 418) und einer weiteren Pause (Z. 419) vorerst die von Gabriele eingeforderte Stellungnahme und äußert dann in Zeile 420 bis 426 ihre positive Bewertung der Performanz einer Schauspielerin, die sie über die von ihr im Stück dargestellten Figuren identifiziert und Gabriele somit als Bewertungsobjekt zugänglich macht.

Nachdem sich Gabriele und Cecilia - angestoßen durch Gabrieles Bewertungseinforderung in Beispiel 66 - darüber verständigt haben, auf welche Figur im Stück sich Cecilias Bewertung bezieht, fordert Gabriele in Beispiel 67 Cecilia explizit zu einer Bewertung der im Theaterstück präsentierten Musik auf (Z. 471):

Beispiel 67: Köln_14-09-23_Sezuan_Gr.1

471 Gabriele: ${ }^{\circ} \mathrm{h}$ wie fIndest du die muSIK?

$472 \quad(0.6)$

473 DI di DI DI-

$474 \quad(0.4)$

475 Cecilia: EHRlich ge[sagt_n bisschen MERKwür]dig.

476 Gabriele: [DI di DI DI- ]

$477 \quad(0.4)$

478 ich find es GANZ TOLL.

479 Cecilia: find_s die GUT? 
480 481
Gabriele: also RICHtig TOLL find ich das.

$(1.6)$

Ihrer Bewertungseinforderung hinsichtlich der Musik im Stück lässt Gabriele in Zeile 473 eine gesangliche Rezitierung einer Melodie folgen, mit der sie auf die von ihr angesprochene Musik referiert, diese so als Bewertungsgegenstand rekonstruiert und einer Bewertung durch Cecilia zugänglich macht. Cecilia liefert mit „EHRlich gesagt_n bisschen MERKwürdig.“ (Z. 475) die von Gabriele eingeforderte Stellungnahme, während Gabriele simultan nochmals die Melodie rezitiert („DI di DI DI“, Z. 476) und mit „ich find es GANZ TOLL.“ in Zeile 478 ebenfalls eine - mit Cecilia nicht übereinstimmende - Bewertung der Musik äußert. Der Cecilias Stellungnahme vorangestellte Disclaimer (vgl. Hewitt/Stokes 1975) „EHRlich gesagt“ (Z. 473) projiziert möglicherweise Nichtübereinstimmung, die durch Gabrieles Bewertung in Zeile 478 auch explizit verbal bestätigt wird. Die Formel „EHRlich gesagt“ dient Cecilia hier als Vorsichtsmaßnahme, indem sie ihre Positionierung gegenüber Gabriele als potenziell heikel und Face-bedrohend markiert (siehe auch Beispiel 35, Kapitel 7.3). Nach Gabrieles sehr positiver Bewertung der Musik in Zeile 478 fordert Cecilia mit ihrer Nachfrage in Zeile 479 eine Bestätigung von Gabriele ein. Gabriele liefert die eingeforderte Bestätigung in Form einer schlussfolgernden Bewertung, in der sie ihre sehr positive Erstbewertung aufgreift, diesmal aber durch die Gradpartikel „RICHtig“ (Z. 480) intensiviert. Eine weitere Reaktion Cecilias bleibt aus.

Auch in Ausschnitt 68 wird ein konkreter Aspekt auf der ästhetischperformativen Ebene des Theaterstücks erfragt. Durch die geschlossene Frage „fandest du die LEHrerrolle au!THEN!tisch?“ (Z. 336), die sie durch die Erläuterung „also wie sie sich verHALten hat?“ expandiert, fordert Annalena von ihrer Gesprächspartnerin Anna explizit eine Antwort hinsichtlich der Inszenierung einer im Stück Verrücktes Blut dargestellten Figur ein:

\section{Beispiel 68: Siegen_14-10-26_Blut_Gr.2}

$\begin{array}{lll}336 & \text { Annalena: } & \text { fandest du die LEHrerrolle au! THEN! tisch? } \\ 337 & & \text { also wie sie sich ver[HALten hat? }] \\ 338 & \text { Anna: } & {[((\text { lacht }))]} \\ 339 & \text { Jasmin: } & {[((\text { lacht }))]} \\ 340 & \text { Pascal: } & \text { axxx }[\text { xxxxxx- }] \\ 341 & \text { Anna: } & {[((\text { lacht }))]{ }^{\circ} \mathrm{h}} \\ 342 & \text { Jasmin: } & {[((\text { lacht }))]} \\ 343 & \text { Anna: } & {[((\text { lacht }))]} \\ 344 & \text { Jasmin: } & {\left[{ }^{\circ} \mathrm{hh}\right.}\end{array}$




$\begin{array}{lll}345 & & (0.2) \\ 346 & \text { Anna: } & \text { oh nein; } \\ 347 & & ((\text { lacht })) \\ 348 & & (0.5) \\ 349 & \text { Pascal: } \quad & \text { also ich GLAUB- } \\ 350 & & (0.3) \\ 351 & & \text { solche leute } \\ & & \text { [werden }(.) \text { nicht LEHrer. }] \\ 352 & \text { Annalena: } & {[\text { [und ICH fand_s auch nich auth] }} \\ 353 & \text { Anna: } & \text { nee- }\end{array}$

Durch die paraverbale Markierung des Adjektivs „au!THEN!tisch“ indiziert Annalena bereits ihre eigene Bewertung der im Stück inszenierten Lehrerrolle, die Anna nach Lachen in Zeile 346 mit einem „nein;“ auch bestätigt. In den Zeilen 349 und 351 äußert Pascal seine Einschätzung, während Annalena in Zeile 352 in Überlappung mit Pascals Äußerung ihre bereits in Zeile 336 paraverbal indizierte negative Bewertung mit „und ICH fand_s auch nich authentisch-“ explizit verbalisiert. Anna bestätigt ihre mit Annalena geteilte Ansicht nochmals in Zeile 353 (,nee“).

Während in den vorangehend angeführten Ausschnitten nach Teilaspekten des Theaterstücks beziehungsweise der Inszenierung gefragt wurde, lenkt Annalena in Ausschnitt 69 den Fokus auf die gesamte Inszenierung und fordert von Hanna explizit eine Globalbewertung ein (Z. 316). Der Ausschnitt entstammt einem Gespräch zwischen Karl, Margarete und Björn sowie Annalena, Elvira und Hanna ${ }^{93}$ in der Pause des Theaterstücks Der Hundertjährige, der aus dem Fenster stieg und verschwand (siehe Kapitel 6.6).

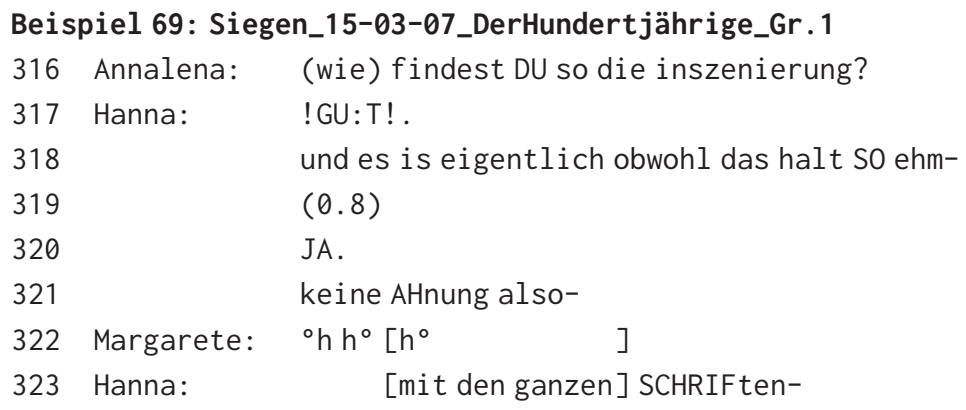

93 Karl, Margarete und Björn bilden eine Gruppe, Annalena, Elvira und Hanna die andere Gruppe. Beim Verlassen des Theatersaals kommen die beiden Gruppen miteinander ins Gespräch und trennen sich erst zu einem späteren Zeitpunkt wieder. 
324

325

326

327 äh und es gibt ja KEIN-

EIgentlich kein wirkliches bühnenbild-

Margarete: ${ }^{\circ} \mathrm{hh}$

Hanna: is_es trotzdem extrem NAH.

In Zeile 317 liefert Hanna mit einem mit Nachdruck geäußerten „!GU:T!.“ die von Annalena zuvor relevant gesetzte Bewertung. In direktem Anschluss an ihre positive Globalbewertung der Inszenierung versucht Hanna, weitere Erläuterungen zu liefern, deren Formulierungen ihr allerdings Schwierigkeiten bereiten: Die Beschreibungen ab Zeile 318 werden von Hanna unsicher hervorgebracht und durch den Heckenausdruck „eigentlich“, den Disfluenzmarker „ehm“ (Z. 318), die Pause (Z. 319) sowie „keine AHnung“ (Z. 321) als Ankündigung epistemischer Unschärfe (vgl. König 2014: 190), die in diesem Beispiel ebenfalls als Disfluenzmarker fungiert, verzögert. Ab Zeile 323 greift Hanna dann mit dem Verweis auf das Bühnenbild einen Aspekt der Inszenierung auf der ästhetischperformativen Ebene heraus und beschreibt vermutlich die von Annalena in Zeile 316 als Bewertungsgegenstand eingeführte Inszenierung, auf die sie mit der Proform „es“ verweist (Z. 327), als „extrem NAH“. Im Anschluss an diese Beschreibung folgt keine weitere Bewertung des Bühnenbilds, sondern ein Vergleich zwischen der literarischen Grundlage und deren Umsetzung im Theaterstück.

Im folgenden Ausschnitt 70 wird bei der Initiierung der Bewertungssequenz ebenfalls eine Globalbewertung - in diesem Fall des Inhalts des Theaterstücks erfragt. Der Ausschnitt entstammt einem Gespräch zwischen Thomas und Marina in der Pause des Theaterstücks Der Kaufmann von Venedig (siehe Kapitel 6.6). Im Vorfeld des angeführten Ausschnitts bewerten Marina und Thomas die Aspekte Bühnenbeleuchtung und Bühnenbild und vergleichen das Bühnenbild der Inszenierung mit dem bereits von ihnen besuchten Theaterstück Romeo und Julia.

Beispiel 70: Siegen_15-02-18_Kaufmann_Gr.3

370 Thomas: wie gefällt dir das stück denn INhaltlich (.) bislang.

$371 \quad(0.7)$

372 Marina: EIgentlich ganz GUT also-

$373 \quad(1.8)$

374 werden sehr viele THEmen aus der ZEIT auch angesprochen $=\mathrm{ja}$.

376 ähm einmal (. ) religiös hier JUdentum CHRIStentum-

377 und dann auch dieser ANtisemitismus und-

$378 \quad(0.7)$ 
379

380

381

382

383 im prinZIP ähm-

(1.3)

kann man das auf die HEUtige zeit auch wieder (. )

äh-

$(0.9)$

Mit seiner Nachfrage „wie gefällt dir das stück denn INhaltlich (.) bislang.“ (Z. 370) fordert Thomas explizit eine Bewertung eines konkreten Aspekts des Theaterstücks von Marina ein. Marina liefert nach kurzem Zögern die durch „EIgentlich“ abgeschwächte und damit in ihrer Gültigkeit eingeschränkte (vgl. König 2014: 195) Bewertung „ganz GUT“ (Z. 372). Nach weiterem kurzen Zögern verbalisiert sie in Zeile 374 ihre vorerst vage gehaltene Beobachtung ,werden sehr viele THEmen aus der ZEIT auch angesprochen ja“. Ab Zeile 376 konkretisiert Marina, durch Disfluenzmarker wie Verzögerungssignale (,ähm“, „äh“), Pausen, Partikeln (,ja“, „hier“), Floskeln (,im prinZIP“) und Vokaldehnungen (,ja::“) hinausgezögert, um welche im Stück angesprochenen Themen es sich handelt und verweist auf den möglichen Übertrag der im Stück präsentierten Inhalte auf die „HEUtige“ gesellschaftliche Wirklichkeit (Z. 379-383).

\subsection{2 (Bewertungs-)Einforderungen durch Bewertungsunterstellung}

Ein weiteres Verfahren, um eine Bewertung einzufordern, ist das der Bewertungsunterstellung. Die Bewertungsunterstellung hat - ähnlich wie die im vorangehenden Kapitel vorgestellte Form der Bewertungsinitiierung durch mehr oder weniger spezifizierte Fragen - die Funktion, eine Bewertung beziehungsweise Stellungnahme vom Gegenüber zu elizitieren. Somit können Bewertungsunterstellungen in den dieser Arbeit zugrunde liegenden Gesprächen als eine Art „fishing device“ (Pomerantz 1980: 188) fungieren. In diesem Zusammenhang spielt auch die von Pomerantz auf der Basis von privaten Alltagsgesprächen beschriebene und bereits in Kapitel 2.3.3 erläuterte Differenzierung zwischen type 1 knowables und type 2 knowables eine tragende Rolle. Denn anders als bei einer selbstinitiierten Erstbewertung, bei der der Sprecher epistemische Autorität und einen direkten (Wissens-)Zugang zum Bezugsobjekt seiner eigenen Bewertung hat (type 1 knowable), verfügt bei einer Bewertungsunterstellung nicht der Sprecher, sondern der Rezipient der Unterstellung über den nötigen Wissenszugang. Was also bei einer Bewertungsunterstellung passiert, kann mit Pomerantz' Worten wie folgt beschrieben werden: 
In what is called fishing, a speaker makes an assertion of a type 2 knowable that refers to an event about which there is a type 1 knowable for the recipient. The ,fishing ' assertion is essentially to be recognized as situated - that is, built out of particular, circumstantial details of an other's activity available to the asserter - and as a product of limited access relative to the recipient's type 1 access as subject-actor.

(Pomerantz 1980: 188)

Dieses Verfahren soll nachfolgend anhand einer expliziten Bewertungseinforderung durch eine Bewertungsunterstellung illustriert werden. Der Ausschnitt entstammt einem Gespräch zwischen Nelli und Wanja in der Pause des Theaterstücks Der Kaufmann von Venedig. Nachdem der Applaus nach dem ersten Teil des Theaterstücks verebbt ist und sich Nelli und Wanja auf den Weg ins Foyer begeben, um sich mit Getränken zu versorgen, initiiert Nelli durch ihre Bewertungsunterstellung „du findest es ja !GANZ! schlimm.“ (Z. 005) die Bewertungsinteraktion und fordert Wanja mit dem angeschlossenen Rückversicherungssignal „ne?“ (Z. 006) zu einer Stellungnahme auf. Für Nelli ist die unterstellte Bewertung ein type 2 knowable, für Wanja hingegen ein type 1 knowable, da es um seine Bewertung beziehungsweise sein Erleben und damit für ihn auch um einen direkten Wissenszugang geht (vgl. auch Heritage/Raymond 2005). Das von Nelli im Anschluss an ihre Bewertungsunterstellung geäußerte Rückversicherungssignal „ne“ fordert Wanja nicht nur explizit zu einer Reaktion auf, sondern indiziert auch, dass Nelli durch ihre Unterstellung Wissen beansprucht, über das in erster Linie Wanja verfügt, das für Nelli selbst aufgrund eines nicht direkten Wissenszugangs aber nur Wissen „zweiter Hand“ darstellt.

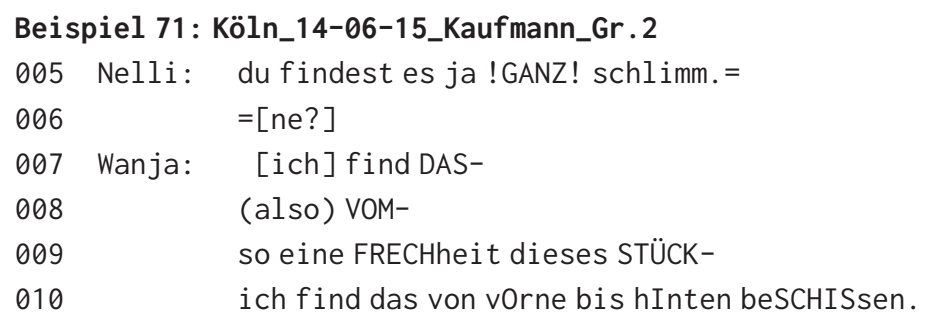

Dass Nellis Versuch der Bewertungseinforderung durch ihre Unterstellung erfolgreich war, wird anhand der von Wanja ab Zeile 007 gelieferten Globalbewertung deutlich. Mit seiner mehrere Abbrüche enthaltenden und affektiv aufgeladenen Äußerung ,ich find DAS- (also) VOM- so eine FRECHheit dieses STÜCK-“ (Z. 007-009) nimmt Wanja eine extrem negative Position hinsichtlich des gesamten Theaterstücks ein und schließt unmittelbar eine weitere extrem formulierte Negativbewertung an, mit der er das im ersten Teil des Theaterstücks Rezipierte als „von vOrne bis hInten beSCHISsen“ (Z. 010) evaluiert. Dass Nelli das Rückversicherungssignal „ne“ an ihre Bewertungunterstellung anschließt und dass ihre 
Unterstellung von Wanja bestätigt wird, weist darauf hin, dass es bereits während der Rezeption beziehungsweise im Vorfeld des Einschaltens des Aufnahmegeräts für Nelli Hinweise auf eine negative Bewertung Wanjas gegeben haben könnte. ${ }^{94}$ Gleichzeitig schreibt Nelli mit dem an ihre Bewertungsunterstellung angeschlossenen Rückversicherungssignal „ne“ Wanja die von ihr mit ihrer vorweggenommenen, unterstellten Bewertung beanspruchte epistemische Autorität nachträglich wieder zu (vgl. Heritage/Raymond 2005). Die Extrembewertung von Wanja wird allerdings vorerst nicht weiter aufgegriffen und bearbeitet, sondern Nelli und Wanja begeben sich auf den Weg ins Theaterfoyer.

\subsubsection{Selbstinitiierte Bewertungen ohne explizite Einforderung einer Zweitbewertung}

Neben der Möglichkeit, Bewertungen vom Gegenüber mittels mehr oder weniger konkreter Fragen sowie durch Bewertungsunterstellungen einzufordern, besteht eine weitere Möglichkeit in einem von den Beteiligten selbst initiierten Einstieg in die Bewertungsinteraktion. Im Folgenden werden selbstinitiierte Bewertungen ohne eine explizite Einforderung einer Zweitbewertung vorgestellt.

In Gesprächsausschnitt $72^{95}$ unterhalten sich Marina, Christine und Sven in der Pause des Theaterstücks Alle sieben Wellen. Der von Marina vorgenommene, durch ihre Bewertung „oh VOLL gu:t- die inszeNIErung.“ (Z. 001-003) selbstinitiierte Einstieg in die Bewertungsinteraktion ist gleichzeitig der Beginn des gesamten Gesprächs.

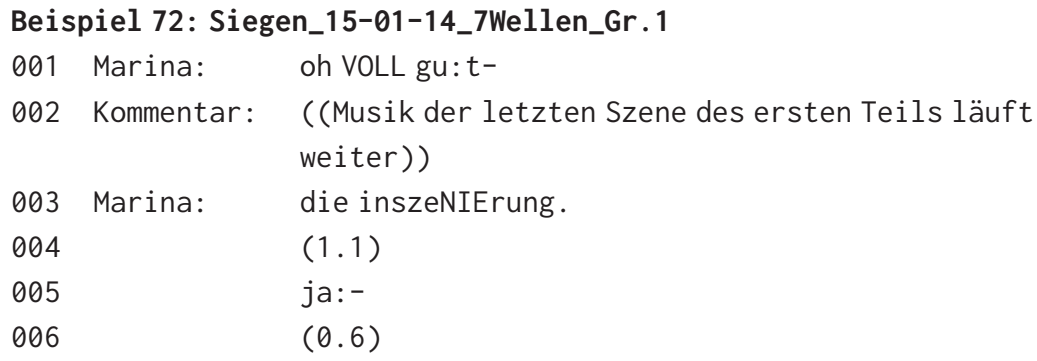

94 Vergleiche König (2017: 241, Herv. Im Original) zu mit „ne“ getaggten Äußerungen: „Dass es in Hörerreaktionen, die auf eine durch ne getaggte Äußerung folgen, eher zu einer Bestätigung als zu einer Ablehnung der Annahmen in der Bezugsäußerung kommt [. . .], soll als Indikator dafür gewertet werden, dass die SprecherInnen gleichzeitig zum Ausdruck bringen, dass sie sich ihrer Äußerung relativ sicher sind, auch wenn dem Gegenüber das Recht zur Bestätigung zugeschrieben wird.“

95 Siehe zu einer früheren Analyse dieses Beispiels Hrncal (2018: 252-254). 


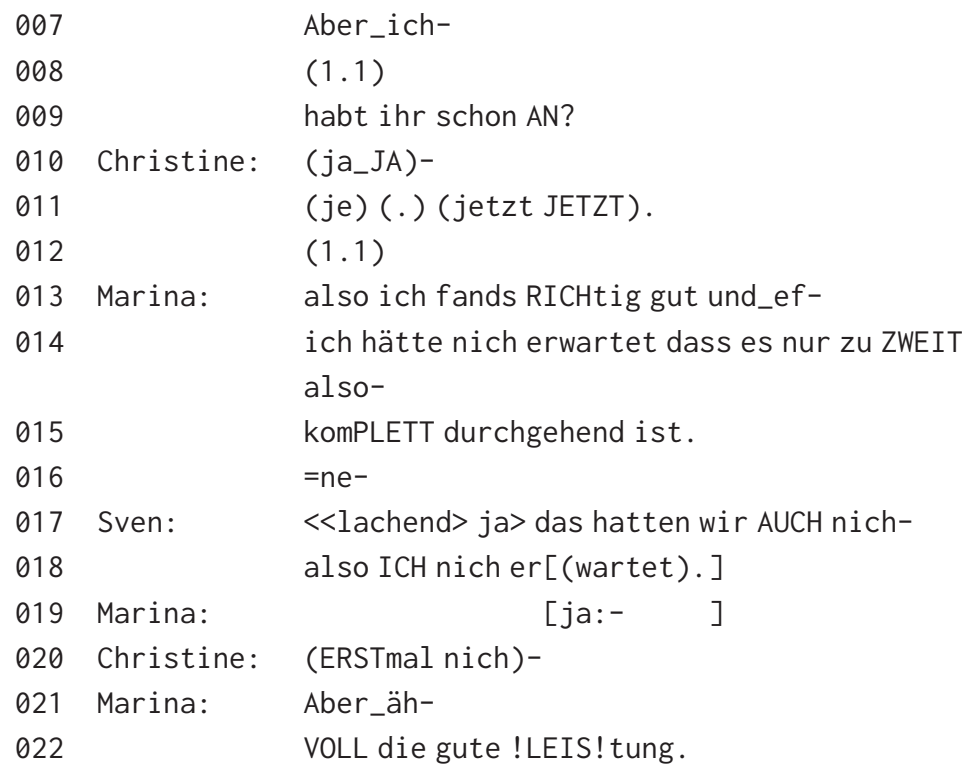

Marina projiziert bereits mit ihrer Bewertung, die die Gradpartikel „VOLL“ sowie das Bewertungsadjektiv „gu:t“ umfasst (Z. 001), ein Bezugsobjekt, dass sie erst mit der nachgeschobenen Expansion „die inszeNIErung“ in Zeile 003 spezifiziert. Im Anschluss projiziert sie in Zeile 007 eine Einschränkung („Aber_ich“) ihrer zuvor geäußerten positiven Globalbewertung, führt ihre Äußerung jedoch nicht zu Ende. Stattdessen kommt es zu einer kurzen side sequence (vgl. Jefferson 1972), in der sich Marina nach einer kurzen Pause bei ihren Gesprächspartnern erkundigt, ob ihre Geräte bereits aufnehmen. Im Anschluss an Christines Bestätigung, dass das Gerät beziehungsweise die Geräte laufen (Z. 010-011), nimmt Marina in Zeile 013 das ursprünglich von ihr durch ihre positive Bewertung eingeführte Thema durch eine resümierende, die Proform „es“ als Bewertungsobjekt enthaltende Bewertung wieder auf. Durch „ich hätte nich erwartet dass es nur zu ZWEIT also- komPLETT durchgehend ist. = ne-“ (Z. 014-016) äußert Marina ihre nicht erfüllten Erwartungen an das Theaterstück, die allerdings nicht zu einer negativen Bewertung führen. Sven stimmt Marina lachend - für sich und Christine als Paar sprechend (Z. 017) - zu, relativiert anschließend jedoch den mit der Bewertung erhobenen Gültigkeitsanspruch, indem er seine Äußerung durch eine eingeschobene Subjektivierung korrigiert (<<lachend $>$ ja $>$ das hatten wir AUCH nich- also ICH nich er(wartet).“, Z. 017-018). Sowohl Marina (,,ja:“, Z. 019) als auch Christine schließen sich Svens Äußerung an (Z. 020). In Zeile 021 und 022 wechselt 
Marina das Bezugsobjekt von der Qualität der Inszenierung hin zur Qualität der schauspielerischen Leistung, die sie auch explizit positiv bewertet.

\subsubsection{Selbstinitiierte Bewertungen mit expliziter Einforderung einer Zweitbewertung}

Während in den im vorangegangenen Kapitel angeführten Gesprächsausschnitten die Beteiligten auf die Möglichkeit zurückgegriffen haben, den Einstieg in die Bewertungsinteraktion mittels einer selbstinitiierten Bewertung ohne explizite Einforderung einer Zweitbewertung zu realisieren, schließen die Beteiligten in den nachfolgenden Gesprächsausschnitten an ihre selbstinitiierten Bewertungen ein Rückversicherungssignal oder eine Frage an und fordern somit von ihrem Gegenüber explizit eine Stellungnahme beziehungsweise Zweitbewertung ein.

Im folgenden Gesprächsausschnitt ${ }^{96}$ unterhalten sich Viktoria und Donata im Anschluss an das von ihnen besuchte Theaterstück Brain and Beauty (siehe Kapitel 6.6), das Schönheitsoperationen thematisiert. Als der Schlussapplaus verklungen ist, fordert Viktoria Donata dazu auf, den Theatersaal zu verlassen. Donata greift Viktorias Aufforderung mit der Frage, ob sie „noch mal RAUSgehn“ (Z. 005) sollen auf und wiederholt das bereits von Viktoria geäußerte „KOMM“ (Z. 003) in Form eines Echos mit gleicher Intonation. Ab Zeile 013 initiiert Donata die Bewertungsinteraktion.

$\begin{array}{lll}\text { Beispiel 73: Köln_14-04-22_BrainAndBeauty_Gr.1 } \\ 001 \text { Viktoria: } & \text { so. } \\ 002 & (0.3) \\ 003 & \text { jetz KOMM. } \\ 004 & (5.1) \\ 005 & \text { Donata: } & \text { sollen wir nochmal RAUSgehn? } \\ 006 & (0.4) \\ 007 & \text { KOMM. } \\ 008 & (1.9) \\ 009 & \text { Viktoria: } \quad{ }^{\circ} \text { hh OH mann- }{ }^{\circ} \mathrm{hh} \\ 010 & & (5.3)\end{array}$

96 Siehe zu einer früheren Analyse dieses Beispiels Hrncal (2018: 254-255) sowie Habscheid (2016: 128-131) mit Fokus auf situierter sprachlicher Bedeutungskonstitution. 


\begin{tabular}{|c|c|c|}
\hline 011 & & uah- \\
\hline 012 & & $(0.3)$ \\
\hline 013 & Donata: & ich hätt ja gern noch EINzelapplaus. \\
\hline 014 & & geHABT. \\
\hline 015 & & $(0.9)$ \\
\hline 016 & & für diese einzelnen DARsteller? \\
\hline 017 & & =da warn ja so_n paar wirklich S[Uper \\
\hline 018 & Viktoria: & {$\left[j a_{-} J A\right.$} \\
\hline
\end{tabular}

Donata verbalisiert, dass sie für einige der im Stück agierenden Darsteller aufgrund ihrer guten Performance „gern noch EINzelapplaus“ (Z. 013) gehabt hätte. Mit dieser Äußerung weist Donata einerseits darauf hin, dass es keine Möglichkeit gab, die Leistung einzelner Schauspieler durch Einzelapplaus zu honorieren, und bewertet dies implizit als negativ. Andererseits nimmt sie in direktem Anschluss die Leistung einzelner Darsteller in den Blick (Z. 016) und bewertet diese explizit positiv („da warn ja so_n paar wirklich SUper“, Z. 017), ohne zu konkretisieren, auf welche Darsteller sie sich bezieht (siehe auch Beispiel 29, Kapitel 7.3). Viktoria reagiert auf Donatas Erstbewertung mit Zustimmung (Z. 017), die sich mit Donatas evaluierendem Adjektiv „SUper“ (Z. 016) überlappt, bevor Donata ihre Bewertung mit der Partikel ,ja“ (Z. 016), die geteiltes Wissen unterstellt, und mit dem Rückversicherungssignal „ne?“ (Z. 016), das eine Stellungnahme Viktorias relevant setzt (vgl. Heritage/Raymond 2005; Fetzer 2000; Goodwin/Goodwin 1992: 164), abgeschlossen hat. Dass Viktoria ihre Zweitbewertung in Überlappung mit Donatas Äußerung realisiert, und dass sie Donata nicht zu einer Konkretisierung der in ihrer Bewertung auftretenden Unschärfe der Referenzen ${ }^{97}$ (,diese einzelnen DARsteller“, „So_n paar“, Z. 016) auffordert, weist darauf hin, dass Donatas Bewertung bereits von Viktoria projiziert wurde (vgl. Goodwin/Goodwin 1987: 25). Die von Donata initiierte Bewertungssequenz, in der die schauspielerische Leistung als Teilaspekt auf der ästhetisch-performativen Ebene des Theaterstücks als Bezugsobjekt in den Fokus gerückt wird, schließt Viktoria durch eine Zweitbewertung in Form von Zustimmung ab.

In Ausschnitt 74, der einem Gespräch zwischen Gudrun und Susanne in der Pause des Theaterstücks Der Kaufmann von Venedig entstammt, initiiert Gudrun durch ihre positive Bewertung der Schauspieler in Zeile 690 eine

97 Zur Wahl von Referenzformen im Rahmen des recipient design vergleiche Deppermann (2015: 8). 
Bewertungsinteraktion, fordert mit einem angeschlossenen „find_ste NICH?“ (Z. 691) Susanne zu einer (bewertenden) Stellungnahme auf und indiziert gleichzeitig eine Präferenz für Übereinstimmung.

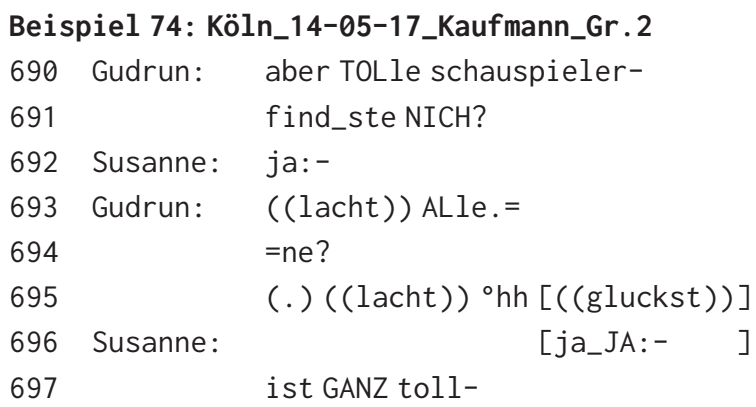

Susanne quittiert Gudruns Erstbewertung mit einem zustimmenden ,ja:“ in Zeile 692, woraufhin Gudrun lachend mit „ALle.==ne?“ (Z. 693-694) die Reichweite ihrer Bewertung betont und durch das Rückversicherungssignal „ne?“ in Zeile 694 eine weitere (übereinstimmende) Stellungnahme beziehungsweise Bestätigung von ihrer Gesprächspartnerin einfordert. Mit einem „ja_JA:“ (Z. 696) und der Gudruns Erstbewertung gleichlaufenden und durch die Gradpartikel „GANZ“ ergänzten und intensivierten Zweitbewertung ,ist GANZ toll““ (Z. 697) stimmt Susanne Gudrun zu und schließt sich ihrer Erstbewertung an.

\subsubsection{Selbstinitiierte Bewertungen mit Bewertungszuschreibung}

Im folgenden Ausschnitt, ${ }^{98}$ in dem sich Gudrun und Susanne in der Pause des Theaterstücks Der Kaufmann von Venedig (siehe Kapitel 6.6) unterhalten, steigt Susanne nach Gudruns Kommentar zu Bühne und Bühnenbild mittels einer selbstinitiierten Bewertung in die Bewertungsinteraktion ein. In diesem Beispiel schreibt die eigentlich Erstbewertende, Susanne, den ihrer Bewertung vorangehenden Äußerungen ihrer Gesprächspartnerin nachträglich den Status einer Bewertung zu.

Beispiel 75: Köln_14-05-17_DerKaufmann_Gr.2

103 Gudrun: hast du son theaterstück schonmal geSEhen mit so ner RIEsigen [LANgen] [BÜHne- ]

104 Susanne: [nä: ] [mit so ner] LANgen

98 Siehe zu einer früheren Analyse dieses Beispiels Hrncal (2018: 255-256). 


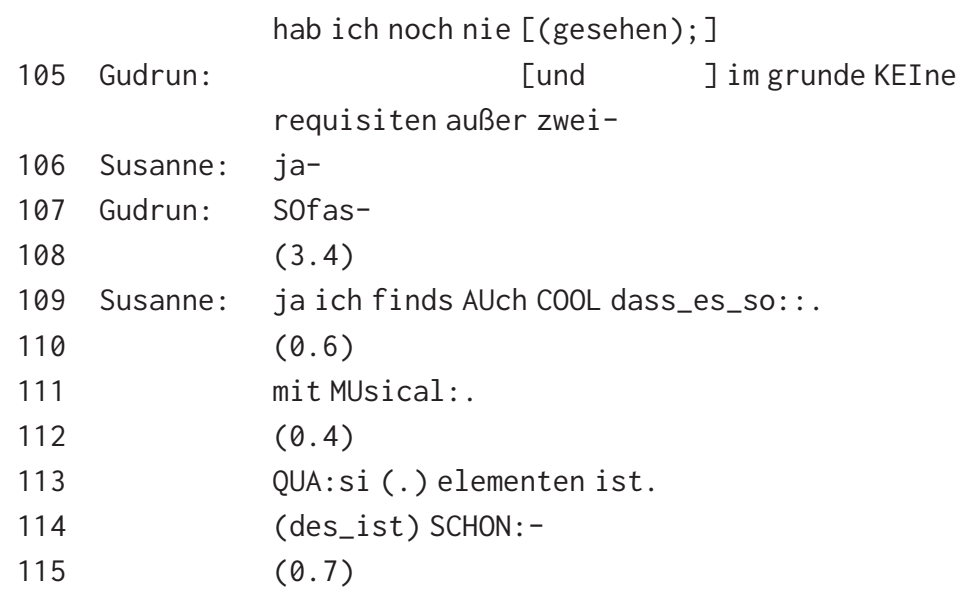

Susannes Bewertung in den Zeilen 109 bis 113 (,ja ich finds AUch COOL dass_es_so::. (0.6) mit MUsical:. (0.4) QUA:si (.) elementen ist.“) weist darauf hin, dass sie die von Gudrun in Zeile 103 realisierte Frage als Bewertungsaufforderung deutet beziehungsweise ihrer Beschreibung der Bühne im Nachhinein aufgrund der paraverbalen Realisierung den Status einer Bewertung zuschreibt (siehe auch Beispiel 41, Kapitel 7.4). Mit ihrer Bewertung wechselt Susanne das von Gudrun mit ihrer Bewertung fokussierte Bezugsobjekt „Bühnenbild“ auf der ästhetisch-performativen Ebene des Theaterstücks und nimmt die Inszenierung des Theaterstücks auf der globalen Ebene in den Blick (,dass_es_so::. (0.59) mit MUsical:. (0.41) QUA:si (.) elementen ist.“, Z. 109-113). Susannes Anschlussäußerung „(des_ist) SCHON:“ (Z. 114) projiziert eine Expansion ihrer Bewertung, die Susanne im Folgenden allerdings nicht zu Ende führt. Die implizite Bewertungsunterstellung Susannes gibt einen Hinweis auf das bereits in Kapitel 2.1.3 angesprochene Problem, dass Beschreibungen und Bewertungen schwer $\mathrm{zu}$ trennen sind und in Beschreibungen auch Bewertungen mitschwingen können, was in diesem Beispiel vermutlich der prosodischen Aufladung der eigentlich beschreibenden Adjektive in Gudruns Äußerungen geschuldet ist.

\subsubsection{Ergebnisse}

Auf Basis der in diesem Kapitel analysierten Gespräche lassen sich drei Verfahren herausstellen, die die Beteiligten in den vorliegenden Gesprächen im Rahmen der Initiierung von Bewertungsinteraktionen nutzen. Diese drei Verfahren werden nachfolgend mit Bezug auf ihre Ausprägungsvarianten in den in Kapitel 8.1 besprochenen Ausschnitten dargestellt. 


\section{Verpflichten}

Einstiege in Bewertungssequenzen können von den Gesprächsbeteiligten durch eine explizit zu einer (bewertenden) Stellungnahme auffordernden Frage in variierenden Stufen der Konkretisierung oder selbstinitiiert realisiert werden. Die Initiierung einer Bewertungsinteraktion mittels der Einforderung einer Stellungnahme vom Gegenüber hat laut Auer und Uhmann (1982: 17) die folgenden zwei Vorteile:

Äußert nun ein Teilnehmer als Bewertungsvorlauf eine Frage, so ,zwingt‘ er den anderen, die Erste Bewertung zu produzieren und das Risiko der Nichtübereinstimmung auf sich zu nehmen. Andererseits nimmt sich der Frager durch ein solches Vorgehen nicht die Möglichkeit, selbst seine Meinung zu äußern, da das Organisationsschema ja als dritten Schritt eine Gegenbewertung vorsieht.

Mit Blick auf die in diesem Kapitel angeführten Analysen ist anzumerken, dass in der eine Stellungnahme einfordernden Frage bereits auf der paraverbalen Ebene eine Bewertung indiziert werden kann (siehe Beispiel 68). Das bedeutet, dass die Stellungnahme beziehungsweise Erstbewertung des Gegenübers nicht notwendigerweise auch die erste Bewertung der Bewertungssequenz ist, sondern dass bereits die Einforderung zu einer Stellungnahme hinsichtlich der prosodischen Konturierung die erste Bewertung darstellen kann.

Etwas anders als bei Einstiegen in Bewertungsinteraktionen durch Fragen sieht es im Fall von Bewertungsunterstellungen aus. Zwar verpflichten (vgl. Auer/ Uhmann 1982: 17) die Beteiligten auch durch diese Form der Initiierung einen Gesprächspartner zu einer Stellungnahme, geben die Erstbewertung und die damit verbundene Gefahr der Nichtübereinstimmung an den Gesprächspartner ab und behalten sich selbst die Möglichkeit vor, mittels einer Zweitbewertung ihre „Meinung“ zu äußern. Allerdings begeben sie sich mit ihrer Unterstellung auch in eine heikle Situation, da eine Zurückweisung beziehungsweise Nicht-Bestätigung der Unterstellung durch das Gegenüber und somit auch eine Face-Bedrohung möglich wäre.

\section{Positionieren}

Neben der Initiierung einer Bewertungsinteraktion durch das Einfordern einer Stellungnahme greifen die Beteiligten zudem auf die Möglichkeit zurück, selbst durch die Äußerung einer Erstbewertung die Bewertungsinteraktion zu initiieren, entweder ohne explizit eine Zweitbewertung einzufordern oder mit einer expliziten Einforderung einer (bewertenden) Stellungnahme durch eine der Erstbewertung nachgeschobene Frage oder ein Rückversicherungssignal wie zum Beispiel ne. Eine nachgeschobene Frage oder ein Rückversicherungssignal kann den Erstbewertenden dazu dienen, die mit ihrer deklarativen Erstbewertung 
beanspruchte epistemische Autorität abzustufen. Die Reaktionen auf diese Form der Erstbewertung (deklarative Bewertung und Rückversicherungssignal) weisen allerdings in den untersuchten Beispielen nicht die von Heritage und Raymond (2005: 30) angeführte Form (Bestätigung mit nachfolgender Zustimmung) ${ }^{99}$ auf, sondern werden zum Beispiel als Zustimmung mit anschließender neuer Erstbewertung, als neue Erstbewertung ohne Zustimmung oder als Erläuterung der Erstbewertung mit anschließender Globalbewertung des Theaterstücks realisiert.

\section{Entschärfen}

In den in diesem Kapitel angeführten Beispielen lässt sich zudem beobachten, dass Einstiege in vielen Fällen in einem scherzhaften Interaktionsmodus (siehe Kapitel 4.1), zum Beispiel durch eine ironische Frage (siehe Beispiel 65), realisiert und lachend oder von Lachen begleitet verbalisiert werden (siehe Beispiel 62, 64, 65, 68, 72, 74). Durch diesen humorvollen Modus indizieren die Beteiligten zum einen den geselligen und vergnüglichen Kontext der Pausengespräche, zum anderen trägt die scherzhafte Realisierungsform der Bewertungen unterstützend zur „Entschärfung“ (siehe Kapitel 4.1) des für die Beteiligten potenziell heiklen Zugzwangs bei, sich hinsichtlich des Theaterstücks zu positionieren oder positionieren zu müssen.

Insgesamt wird anhand der Ausschnitte deutlich, dass das Bewerten des Theaterstücks in den vorliegenden Gesprächen von den Beteiligten als eine zentrale Aufgabe beziehungsweise ein zentrales Merkmal zur Realisierung des Pausengesprächs behandelt, als Ressource zur Orientierung genutzt und erwartet sowie von ihnen offensichtlich als geteilte Praxis vorausgesetzt wird (siehe Kapitel 2.3.2). Dafür spricht einerseits die Tatsache, dass bei einem überwiegenden Teil der Gespräche der Einstieg in die Bewertungsinteraktion und der Einstieg in das Pausengespräch zusammenfallen oder die Bewertungsinteraktion in unmittelbarem Anschluss an das gegenseitige Absichern der Aufnahme oder die gemeinsame Verständigung über den für das Gespräch anvisierten

99 Vergleiche Heritage und Raymond (2005: 30) zu den Praktiken, mit denen Sprecher die mit einer Zweitbewertung verbundenen epistemischen Ansprüche relativ zu den in Erstbewertungen enthaltenen Ansprüchen hochstufen können: „Reviewing these practices, the first - [confirmation + agreement] - is relatively specialized to environments in which first assessments are downgraded by using tag questions. For these reasons, this practice is normally used in circumstances where the speakers achieve alignment concerning their relative rights to assess, and therefore is relatively ,mild“.“ 
Zielort beim Verlassen des Theatersaals inittiiert wird, obwohl die an der Erhebung teilnehmenden Theaterbesucher im Vorfeld der Aufnahme nicht darüber informiert wurden, dass das Bewerten einen Untersuchungsfokus im Rahmen des Projekts darstellt. Andererseits geben die Beobachtung, dass durch einen selbstinitiierten Einstieg in die Bewertungsinteraktion den eigentlich beschreibenden vorangehenden Äußerungen des Gegenübers der Status einer Erstbewertung zugeschrieben wird (siehe Beispiel 75), einen Hinweis darauf, dass aus der Beteiligtenperspektive dem Bewerten im vorliegenden Kontext der Kunstkommunikation ein hoher Stellenwert beigemessen wird, ebenso wie die Reaktionen der Gesprächspartner auf Einforderungen einer Stellungnahme mit einer ersten Bewertung als Antwort auf die offene Ein-Wort-Frage „und?“ oder mit einer Zweitbewertung als Reaktion auf eine Erstbewertung.

\subsection{Sequenzielle und thematische Verläufe von Bewertungsinteraktionen}

Im Anschluss an die Betrachtung möglicher Einstiege in die Bewertungsinteraktion werden im Folgenden sequenzielle und thematische Verläufe von Bewertungsinteraktionen analysiert. Es wird herausgestellt, inwiefern sich die in Kapitel 2 skizzierten, in der interaktionslinguistischen Forschung auf Basis privater Alltagsgespräche und institutioneller Kommunikation herausgestellten sequenziellen Verlaufsstrukturen auch in den dieser Arbeit zugrunde liegenden Pausengesprächen zeigen sowie auf welche Art und Weise die am Gespräch Beteiligten Bewertungen gemeinsam produzieren und aushandeln.

Verbunden mit den sequenziellen Verläufen rückt auch die Themenbearbeitung innerhalb der Bewertungsinteraktionen in den Mittelpunkt. Dabei geht es vor allem um die Frage, wie die Beteiligten die Bezugsobjekte ihrer Bewertungen durch Verweise auf die inhaltliche Ebene (zum Beispiel mit Fokus auf die Handlung und darin agierende Figuren), die ästhetisch-performative Ebene (zum Beispiel hinsichtlich des Bühnenbilds, der schauspielerischen Performance oder der Musik) und die globale Ebene des Theaterstücks (mit Blick auf die gesamte Inszenierung) wechseln. Des Weiteren ist von Interesse, inwiefern durch Verweise auf eine allgemeine gesellschaftliche Ebene (zum Beispiel durch Bezüge auf Personen in ihrer persönlichen Alltagswirklichkeit) möglicherweise Inhalte des Theaterstücks auf die gesellschaftliche Wirklichkeit übertragen werden und dies gegebenenfalls auch zu Moralisierungen führt (vgl. dazu auch Hrncal/ Gerwinski 2015). 


\subsubsection{Bewertungen auf der inhaltlichen und ästhetisch-performativen Ebene des Theaterstücks}

Zunächst wird in diesem Kapitel in den Blick genommen, wie die an den Gesprächen Beteiligten Bewertungsgegenstände auf der inhaltlichen sowie der ästhetisch-performativen Ebene des Theaterstücks konstruieren und ihre Bewertungen hinsichtlich dieser Bezugsobjekte abgleichen.

\subsubsection{Abgleich individuellen Erlebens als Grundlage für Bewertungen} Im folgenden Beispiel ${ }^{100}$ unterhalten sich Victoria und Ulrich in der Pause des Theaterstücks Der gute Mensch von Sezuan (siehe Kapitel 6.6). Dem Ausschnitt geht eine Gesprächspassage voran, in der sich Victoria und Ulrich mit Lola und Timo, die auch zur Besuchergruppe gehören, negativ über die vielen Raucher im Theaterpublikum und in der ganzen Stadt äußern. Nachdem sich die Gruppenteilnehmer daran anschließend über ihre Getränkewünsche verständigt haben, eröffnet Victoria mit einer Bewertung der Länge des Stücks die Bewertungsinteraktion:

Beispiel 76: Köln_14-10-02_Sezuan_Gr.1

$\begin{array}{lll}221 & \text { Victoria: } & \text { für !MICH! war_s natürlich wieder_s } \\ & <<\text { lachend }>\text { glEIche> } . \\ 222 & & <\text { lachend }>\text { weisch>? } \\ 223 & & \text { war BISSL- } \\ 224 & & (0.2) \\ 225 & & \text { zu LANG- } \\ 226 & & (2.1) \\ 227 & & \text { oh aber isch gut geMACHT-= } \\ 228 & & =\text { gell? } \\ 229 & & (0.7) \\ 230 & \text { Ulrich: } & \text { joa? } \\ 231 & & (0.5) \\ 232 & & \text { ich find vor ALlem so diese- } \\ 233 & & (0.4) \\ 234 & & \text { die kuLISsen sind originell; } \\ 235 & \text { Victoria: } & \text { JA_a:- } \\ 236 & \text { Ulrich: } & \text { [ALso (.) äh äh- ] } \\ 237 & \text { Victoria: } & \text { [(ne: und so schön] KURZweilig). }\end{array}$

100 Siehe zu einer früheren Analyse dieses Beispiels Hrncal (2018: 263-265). 


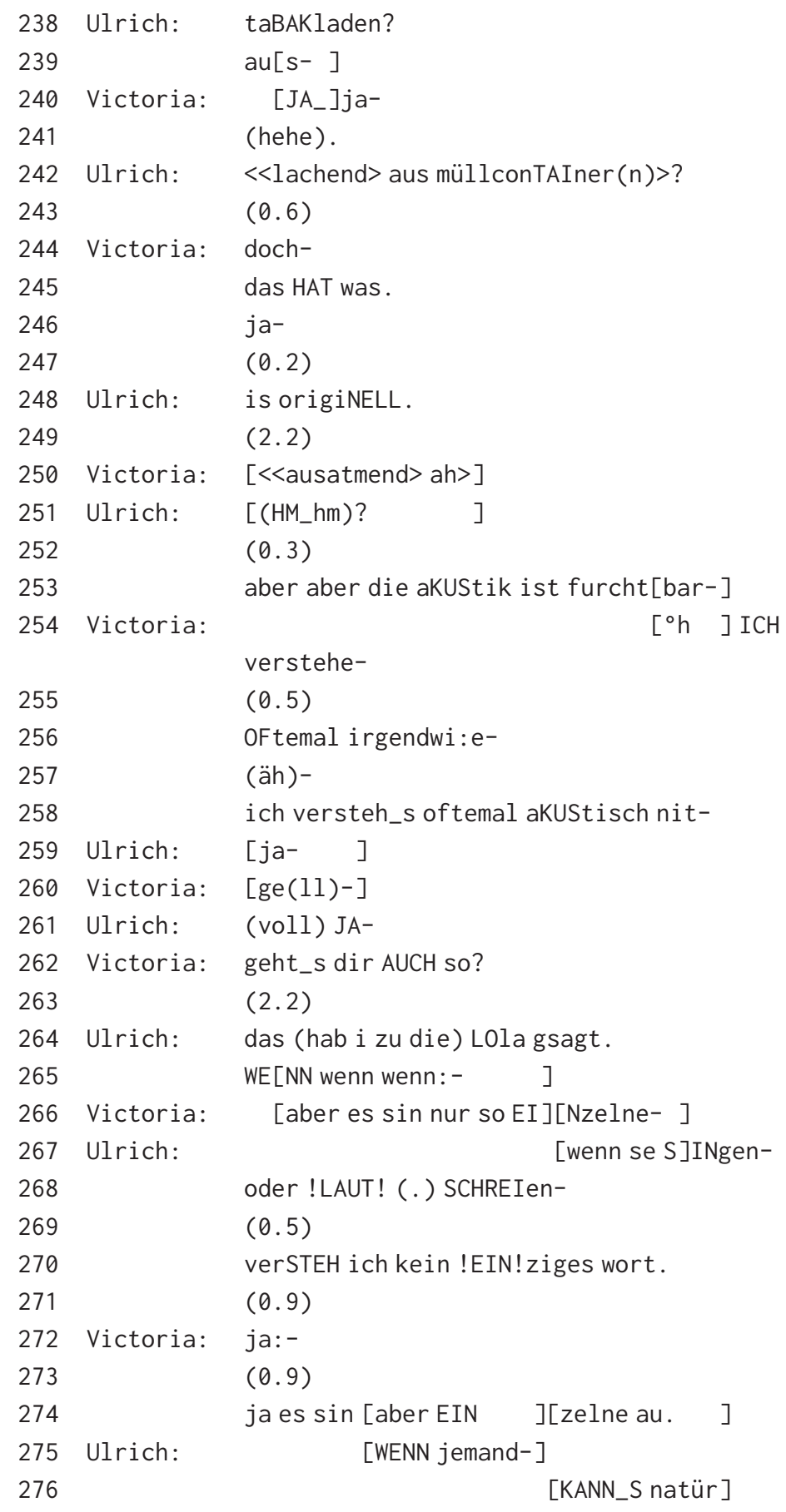




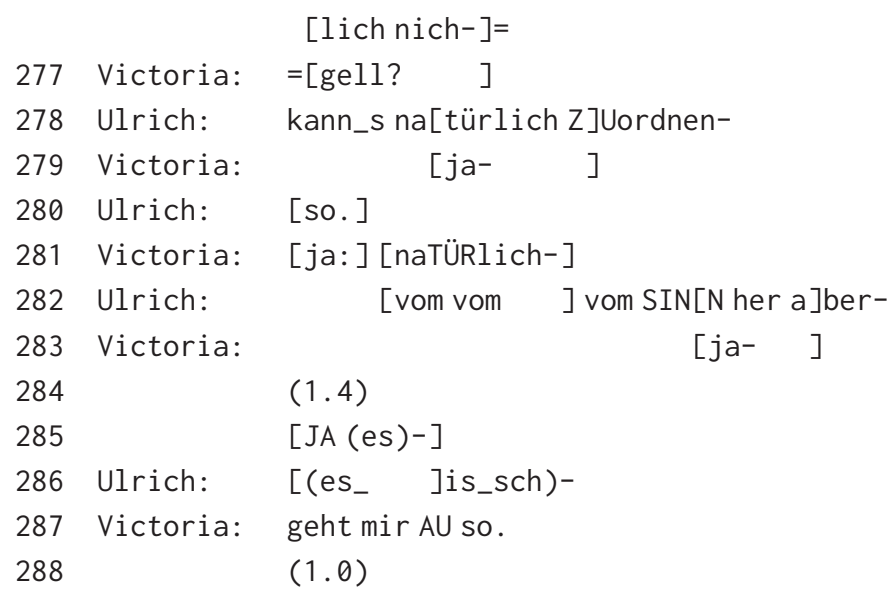

$\mathrm{Zu}$ Beginn der Bewertungssequenz unterstellt Victoria Ulrich mit ihrer Äußerung „für !MICH! war_s natürlich wieder_s <<lachend > glEIche> . <<lachend> weisch>?“ (Z. 221-222) der Interaktion zugrunde liegendes gemeinsames Hintergrundwissen und verweist damit auch auf den Beziehungsstatus der beiden Gesprächspartner. Gleichzeitig projiziert sie mit dieser Äußerung Erläuterungen, die sie mit der durch den Heckenausdruck „BISSL“ (Z. 223) abgeschwächten und durch das eine unverhältnismäßig hohe Ausprägung indizierende Adverb „zu“ (Z. 225) negativ konnotierten Bewertung mit Bezug auf die Länge des Theaterstücks als Bewertungsgegenstand anschließend liefert. Als eine Reaktion Ulrichs auf Victorias Erstbewertung ausbleibt, realisiert Victoria - den Bewertungsgegenstand von der Länge des Theaterstücks hin zur Inszenierung wechselnd - nach einer kurzen Pause (Z. 226) im Kontrast zu ihrer ersten Bewertung die mit der Konjunktion „aber“ eingeleitete deklarative positive Bewertung „isch gut geMACHT-“ (Z. 227-228), die durch ihre Realisierungsform zudem Allgemeingültigkeitsanspruch indiziert. Auffällig ist hier, dass, während Victoria in ihrer ersten Bewertung noch mit der Proform „es“ auf den Bewertungsgegenstand verweist und ihre Bewertung durch diese Unschärfemarkierung als Vorsichtsmaßnahme weniger angreifbar macht, sie in ihrer zweiten Bewertung die Proform „es“ tilgt und diese somit nur indirekt impliziert. Auch die in der ersten Bewertung explizit prosodisch hervorgehobene subjektive Perspektivierung (,!MICH!“, Z. 221) ist in Victorias zweiter Bewertung aufgehoben. Durch das ihrer Bewertung unmittelbar angeschlossene, Präferenz für Übereinstimmung indizierende Rückversicherungssignal „gell?“ (Z. 228) lädt sie Ulrich zu einer Bestätigung ein, stuft die von ihr durch die deklarative Bewertung beanspruchte epistemische Autorität herab und schreibt in diesem Zuge Ulrich die gleichen epistemischen Rechte zur Bewertung des ihm durch 
die Rezeption ebenso zugänglichen Bewertungsgegenstands zu (vgl. Heritage/ Raymond 2005: 23; siehe auch Kapitel 2.3.3). Ulrich reagiert auf Victorias Bestätigungseinforderung vorsichtig mit einem steigend intonierten „joa?“ (Z. 230), das zunächst auch als Nichtübereinstimmung projizierendes Vorlaufelement gedeutet werden könnte. Bei seiner nach einer kurzen Pause realisierten, als subjektiv gerahmten positiven Bewertung ,ich find vor ALlem so diese- (0.4) die kuLISsen sind originell;“ (Z. 232-234) wechselt Ulrich den Bewertungsgegenstand auf der globalen hin $\mathrm{zu}$ einem Bewertungsgegenstand auf der ästhetisch-performativen Ebene. Hier zeigt sich die bereits in Kapitel 8.1 dargestellte sequenzielle Struktur, auf eine Erstbewertung mit (vorsichtiger) minimaler Zustimmung und einer neuen Erstbewertung mit einem Wechsel des Bewertungsgegenstands zu reagieren. Nach Victorias zustimmender Rückmeldung in Zeile 235 liefert Ulrich weitere Erläuterungen zur Begründung seiner Bewertung, indem er auf die Gestaltung des Bühnenbilds verweist („ALso (.) äh äh- (. . .) taBAKladen? aus- (. . .) <<lachend > aus müllconTAIner>?“, Z. 236-236). In Zeile 237 äußert Viktoria in Überlappung mit Ulrich vermutlich eine weitere positive Berwertung („(ne: und so schön KURZweilig).“), bei der das Bezugsobjekt nicht eindeutig indentifizierbar ist und die im weiteren Verlauf auch nicht mehr aufgegriffen wird. Victoria leitet durch ihre schlussfolgernde Bewertung „doch- das HAT was. ja-“ (Z. 244-246), ${ }^{101}$ mit der sie sich Ulrichs Bewertung anschließt, den Abschluss der von ihr initiierten Bewertungssequenz ein. Ulrich wiederholt daraufhin in Zeile 248 nochmals seine Bewertung hinsichtlich des Bühnenbilds, auf die Victoria nicht mehr weiter eingeht und damit die Bewertungssequenz als abgeschlossen behandelt.

Nachdem das Gespräch ins Stocken gerät (Z. 249-252), initiiert Ulrich in Zeile 253 eine neue Bewertungssequenz, indem er, ähnlich wie Victoria in Zeile 227, eingeleitet mit der Konjunktion ,aber“ und somit im Kontrast zu seiner vorherigen positiven Bewertung, die Akustik als Bezugsobjekt auf der ästhetischperformativen Ebene in den Fokus rückt und negativ bewertet. Victoria reagiert auf Ulrichs Erstbewertung nicht mit einer Zweitbewertung der Akustik, sondern verbalisiert akustische Verständnisschwierigkeiten (Z. 258). Ulrich liefert die von Victoria durch ein ihrer Schilderung angeschlossenes „gell“ eingeforderte Bestätigung sowohl in Überlappung mit Victorias Rückversicherungssignal als auch im Anschluss daran (Z. 261). Daraufhin fordert Victoria mit „geht_s dir AUCH so?“ (Z. 262) nochmals eine Bestätigung ihrer Äußerung von Ulrich ein.

101 Vergleiche Heritage und Raymond (2005: 26): „speakers achieve epistemic alignment by downgrading rights to assess in first position and upgrading them in second position. The [confirmation + agreement] format is most commonly used in response to interrogatively formed assessments, particularly those deploying tag questions.“ 
Dieser konkretisiert ab Zeile 265 die Stellen im Theaterstück, an denen er Inhalte akustisch nicht verstehen konnte („WENN wenn wenn:- (. . .) wenn se SINgen- oder !LAUT! (.) SCHREIen- (0.5) verSTEH ich kein !EIN!ziges wort.“). Victoria verweist überlappend mit Ulrichs Erläuterungen darauf, dass „nur so EINzelne“ (Z. 266) schlecht zu verstehen sind und grenzt so Ulrichs verallgemeinernde Referenz auf alle Schauspieler (,se“) auf eine bestimmte Anzahl von Schauspielern ein. Ab Zeile 267 führt Ulrich aus, dass er zwar „vom SINN her“ (Z. 282) zuordnen kann, was gesagt wird, es ihm aber schwer fällt (Z. 286). Victoria stimmt Ulrich mit „geht mir AU so.“ (Z. 287) zu. Nach einer Pause (Z. 288) wird der durch Ulrichs Bewertung initiierte Wahrnehmungsabgleich von beiden Beteiligten als abgeschlossen behandelt.

Wenig später, als Ulrich und Victoria anstehen, um sich mit Getränken zu versorgen, initiiert Ulrich mit seiner äußerungsfinal subjektivierten und durch den Heckenausdruck „n bissel“ abgeschwächten Bewertung „und die !GÖT!ter sind_n bissel diletTANtisch FIND ich.“ (Z. 319) eine weitere Bewertungssequenz, in der er einige der im Stück agierenden Figuren auf der inhaltlichen Ebene als Bezugsobjekt seiner Bewertung in den Fokus rückt:

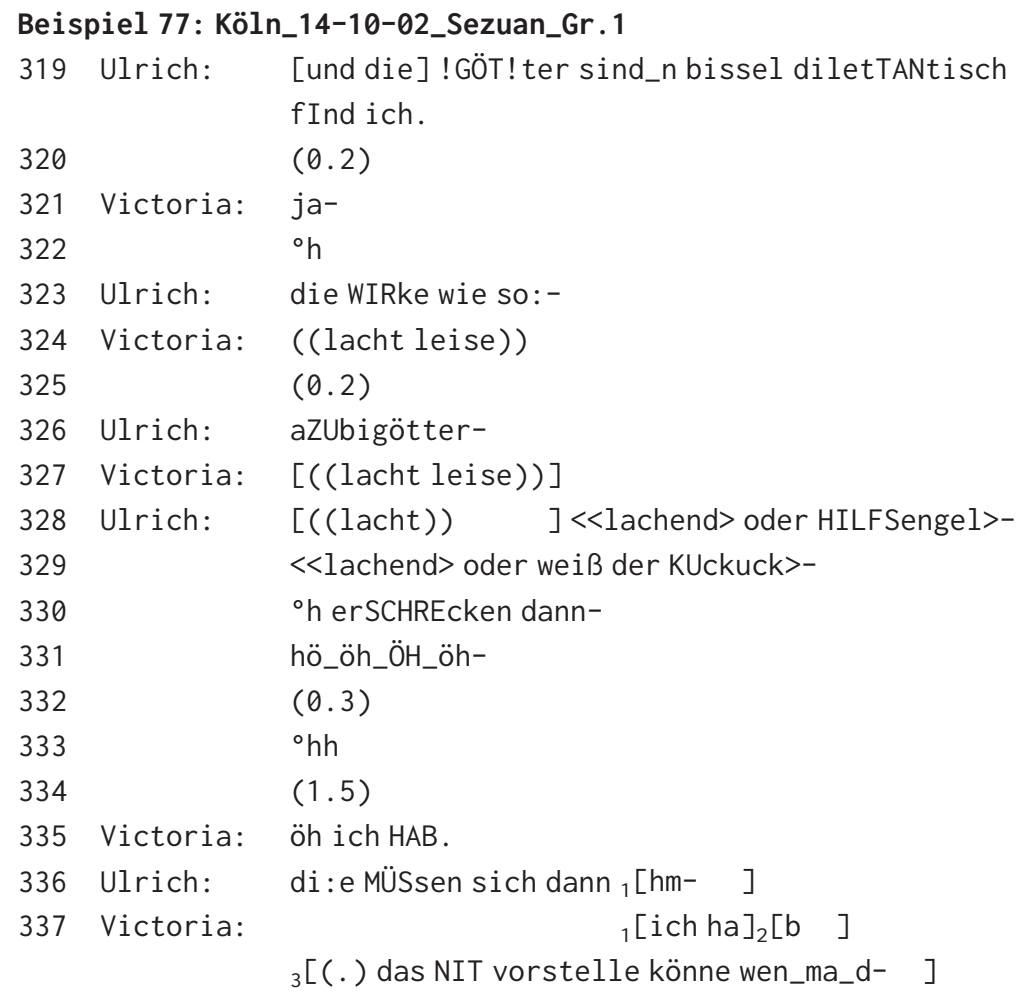




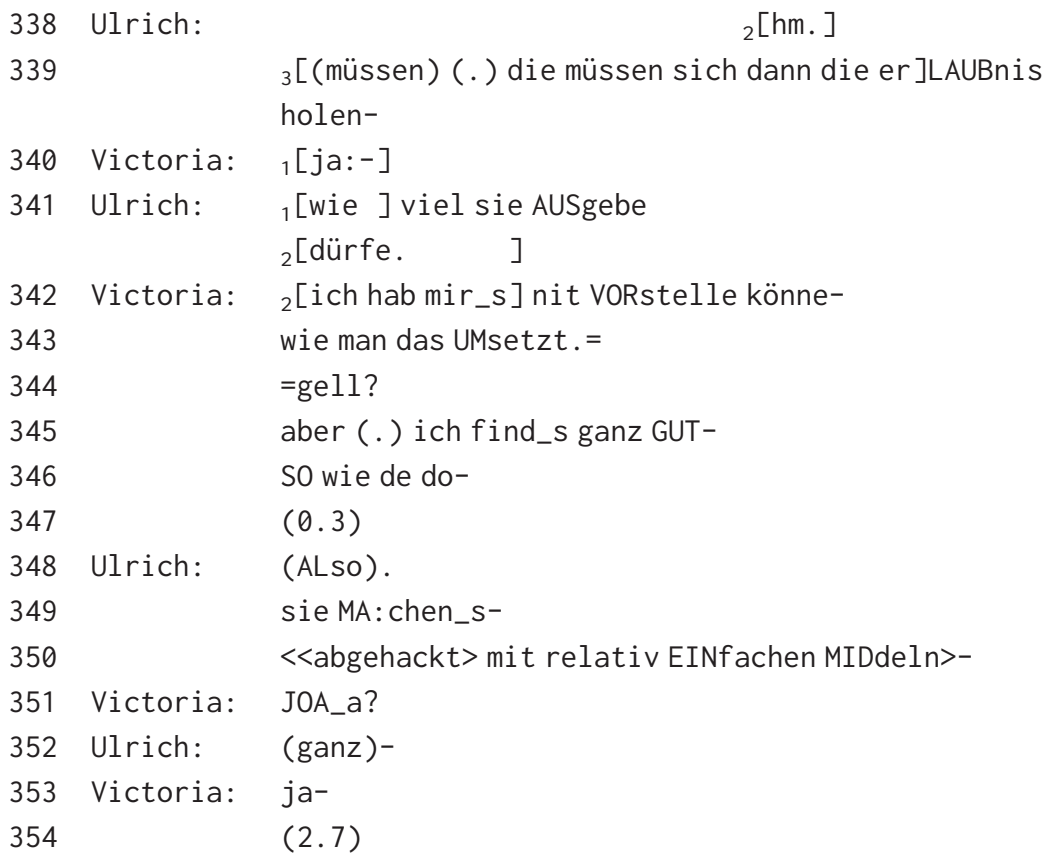

Victoria quittiert Ulrichs Bewertung mit einem ,ja“ (Z. 321), das prosodisch eher einer Hörerrückmeldung als einer Zustimmung gleicht. Ulrich beginnt in Zeile 323 mit weiteren, teils von Lachen begleiteten Erläuterungen seiner Bewertung und bezeichnet die im Stück dargestellten Götter als „aZUbigötter“ (Z. 326) und „HILFSengel“ (Z. 328) „oder weiß der KUckuck“ (Z. 329). Dieser Beschreibung der Götter folgen ab Zeile 330 weitere, bruchstückhafte Erläuterungen, im Rahmen derer Ulrich mit „hö_öh_ÖH_öh“ (Z. 331) Figuren und deren Agieren im Stück zu reinszenieren scheint (siehe auch Beispiel 50, Kapitel 7.4), damit die betreffende Stelle, auf die er mit seiner Reinszenierung verweist, rekonstruiert und somit Victoria zugänglich macht. Dass Ulrich auf die Reinszenierung der Götter zurückgreift, könnte zum einen darauf hinweisen, dass er Schwierigkeiten hat, die Stelle im Stück, auf die er referiert, möglichst präzise zu beschreiben, zum anderen könnte es darauf hindeuten, dass Reinzenierungen den Beteiligten die Möglichkeit bieten, das Bezugsobjekt der Bewertung den anderen Gesprächsteilnehmern möglichst plastisch vor Augen $\mathrm{zu}$ führen. In Zeile 337 setzt Victoria parallel zu Ulrichs Ausführungen zu einer Äußerung an, die sie vorerst abbricht und in Zeile 334 nochmals aufgreift und vervollständigt. Victoria merkt an, dass sie es sich ,nit VORstelle“ kann, ,wie man das UMsetzt.“, setzt mit dem ihrer Anmerkung nachgeschobenen Rückversicherungssignal „gell“ zwar eine Reaktion Ulrichs relevant, fährt dann aber unmittelbar 
mit ihrer positiven, durch die Gradpartikel „ganz“ eingeschränkten und durch die kontrastivierende Konjunktion „aber“ eingeleiteten Bewertung „ich find_s ganz GUT-“ (Z. 345) fort, die sie zudem äußerungsinitial subjektiv perspektiviert. Dabei wechselt sie das Bezugsobjekt auf der ästhetisch-performativen Ebene hin zur globalen Ebene des Theaterstücks. Weitere Erläuterungen zu ihrer Bewertung in Zeile 346 bricht sie allerdings ab. Nach einer Minimalpause bietet Ulrich mit seinem Verweis auf die Art und Weise der Inszenierung von im Stück agierenden Figuren („mit relativ EINfachen MIDdeln“, Z. 350) ein Beschreibungsangebot an, das Viktoria bestätigt. In Zeile 352 setzt Ulrich zu einer Äußerung an, führt diese aber nicht weiter aus. Nach einer weiteren Rückmeldung Victorias und einer Pause wird das Thema von den beiden vorerst als abgeschlossen behandelt, und beide widmen sich ihrer bevorstehenden Getränkebestellung. Erst im späteren Gesprächsverlauf greift Ulrich die Bewertung der Götter nochmals auf, als zwei weitere Gruppenmitglieder, Timo und Lola, wieder dazustoßen. Auch in diesem Beispiel folgt auf die Erstbewertung Ulrichs keine Zweitbewertung durch Victoria. Erst nach Ulrichs Rekonstruktion des im Stück Präsentierten realisiert Victoria eine Bewertung, bei der sie allerdings das Bezugsobjekt wechselt. $\mathrm{Zu}$ beobachten ist im Verlauf dieser Interaktionssequenz, wie beide Gesprächspartner sich über ihr Erleben während der Rezeption des Theaterstücks verständigen („die WIRke wie so:“, Z. 323; „ich hab mir_s nit VORstelle könne“, Z. 342) und so einen gemeinsamen Hintergrund für weitere Bewertungen oder Beschreibungen konstruieren.

Die beiden folgenden Ausschnitte entstammen dem Gespräch zwischen Annalena, Anna, Jasmin und Pascal im Anschluss an das Theaterstück Verrücktes Blut (siehe auch Beispiel 68) und illustrieren, wie die Beteiligten eine Bewertung der Lichteffekte auf der ästhetisch-performativen Ebene des Theaterstücks als Anstoß zur Erläuterung ihrer Deutungen der im Theaterstück präsentierten Inhalte nutzen. Unmittelbar vor diesem Gesprächsaussschnitt unterhalten sich Anna und Annalena über die ihrer Meinung nach teilweise unpassenden Reaktionen des Publikums während der Inszenierung. Nach einer kurzen Pause rückt Anna mit ihrer Bewertung der Ausleuchtung der Bühne einen neuen Aspekt in den Fokus:

\section{Beispiel 78: Siegen_14-10-26_Blut_Gr.2}

238 Anna: und ich fand_s auch COOL mit den ! LICH! tern.

$239=a l s o$ ich hab das ERST nicht richtig durchSCHAUT?

240 Aber (äh)-

241 s GINgen ja immer die-

$242{ }^{\circ} \mathrm{h}$ LICHter an- 
je nachDEM ob [die] jetzt gerade in diesem äh !SCHIL! lerstück sind-

244 Pascal: $\quad[\mathrm{ja}-]$

245

Ode:r(.) [(nicht).]

246 Anna: [oder ] halt äh sozusagen $<<$ lachend $>$ in dem [ANdern] stück>.

247 Pascal: [HM_hm?]=

248 Anna: =also es WAR ja dann letztendlich ! AUCH! irgendwie n the! $\mathrm{A}$ ! ter. $=$

260 Jasmin:

271 Jasmin: (aber ich [mein irgendwie VIEL)]=ne?

272 Anna:

=aber DAS fand ich voll interesSANT.

${ }^{\circ} \mathrm{h}$ am ANfang hab ich gedacht-

OKEE-

zwischendurch schalten die LICHter um.

${ }^{\circ} \mathrm{h}$ und DANN hab ich irgendwie überlegt-

oKEE . =

=wann SCHALten denn die lichter eigentlich umund DANN war_s halt irgendwie soja je nachdem wo die geRAdein welcher ROLle die gerade irgendwie STEcken. (0.5)

aber zwischendurch fand ich das auch exTREMbei EInem_malwurd ich auf einmal so ! VOLL! von dem licht geBLENdet. $=$ =weil_s irgendwie GANZ dunkel war?

$(0.4)$

Anna: ja:-

Jasmin: aBE:R-

an sich fand ich das mit den lichteffekten eigentlich ! AUCH! g-= =also WAR ja auch gut$(0.2)$

[und zum SCHLUSS- ] zum SCHLUSS waren nämlich dann ! AL! le lichter an.= =deswegen war das wahrscheinlich so $\mathrm{n}$ bisschen um das nochmal irgendwie zu verEInen oder so. keine AHnung-

Jasmin: hm- 
Im Anschluss an ihre positive Bewertung (,und ich fand_s auch COOL mit den !LICH!tern.“, Z. 238) verbalisiert Anna affektiv aufgeladen ihr Erleben beziehungsweise Deutungsschwierigkeiten hinsichtlich der Funktion der Lichteffekte während der Rezeption des Theaterstücks (,also ich hab das ERST nicht richtig durchSCHAUT?“, Z. 239). Ab Zeile 240 schildert sie ihre Beobachtungen („Aber (äh)- s GINgen ja immer die- ${ }^{\circ} \mathrm{h}$ LICHter an- je nachDEM ob die jetzt gerade in diesem äh !SCHIL!ler stück sind- (. . .) oder halt äh sozusagen <<lachend> in dem ANdern stück>.“), rekonstruiert damit die betreffende Stelle im Stück und macht ihre Beobachtungen so den anderen Gesprächsteilnehmern zugänglich. In Zeile 248 kommt Anna dann zu ihrem Fazit „also es WAR ja dann letztendlich !AUCH! irgendwie n the!A!ter.“ und schließt ihre Ausführungen mit der kommentierenden Bewertung ,aber DAS fand ich voll interesSANT.“ (Z. 249) ab. Sowohl bei ihrer fazitierenden Deutung als auch bei ihrer Bewertung referiert sie jeweils mit einer Proform-Variante („es“/„das“) auf die gesamte vorherige Äußerungssequenz als Bewertungsgegenstand. ${ }^{102}$ Durch die Wahl des nicht eindeutig negativ oder positiv konnotierten Adjektivs ,interesSANT“ umgeht Anna zudem eine potenziell angreifbare eindeutige Positionierung. Im Anschluss an ihre kommentierende Bewertung (vgl. Auer 1993: 209) führt sie ab Zeile 250 - immer noch mit Bezug zum eingangs des Ausschnitts von ihr eingeführten Bewertungsgegenstand - einen reflektierenden inneren Denkprozess (siehe Kapitel 3.1) beziehungsweise deutenden Gedankengang aus und lässt Annalena, Jasmin und Pascal an ihrem Erleben teilhaben (siehe auch Beispiel 52, Kapitel 7.5). Nach einer kurzen Pause bringt sich Jasmin ins Gespräch ein und reagiert auf Annas Ausführungen und ihre positive Bewertung des Lichts mit der einen Kontrast indizierenden und durch die prosodische Konturierung implizit negativen Bewertung ,aber zwischendurch fand ich das auch exTREM-“ (Z. 260). Diese begründet sie dann durch ihre Wahrnehmungsschilderung „bei EInem_mal- wurd ich auf einmal so !VOLL! von dem licht geBLENdet. = = weil_s irgendwie GANZ dunkel war?“ (Z. 261-263). Dabei grenzt sie die Stelle im Stück, auf die sie verweist, nur sehr vage ein („zwischendurch“, „bei EInem_mal“, „irgendwie GANZ dunkel“), macht ihren Bezug aber scheinbar in ausreichendem Maße für die anderen Gesprächsteilnehmer nachvollziehbar, da diese keine Spezifizierung einfordern. Anna quittiert dies mit dem Rückmeldesignal „ja:“ (Z. 265), bevor sich Jasmin mit ihrer durch den Heckenausdruck „eigentlich“ als unscharf markierten Bewertung „aBE:R- an sich fand ich das mit den lichteffekten eigentlich !AUCH! g- = = also WAR ja auch

102 Vergleiche zur Häufigkeit der Korrelatworte es und das im Zusammenhang mit Bewertungen auch Imo (2012: 233). 
gut-“ (Z. 267-268) Annas Erstbewertung anschließt und sich von ihrer vorherigen impliziten negativen Bewertung distanziert. Anna quittiert dies abermals mit einem zur Kenntnis nehmenden „ja“ (Z. 270), woraufhin Jasmin nochmals sehr vage und im Kontrast (,,aber“, Z. 271) zu ihrer vorherigen positiven Bewertung darauf hinweist, dass es „(irgendwie VIEL)“ war und mit dem Rückversicherungssignal „ne?“ (Z. 271) Anna dazu einlädt, ihre Einschätzung zu bestätigen. Diese Bestätigung liefert Anna allerdings nicht, sondern greift in Überlappung mit Jasmins Äußerung eine bestimmte Stelle im Stück heraus (,zum SCHLUSS (. . .) nämlich dann !AL!le lichter an“, Z. 273) und bietet mit „deswegen war das wahrscheinlich so: bisschen um das nochmal irgendwie zu verEInen oder so.“ (Z. 274) einen als Vermutung gerahmten Deutungsversuch an, den sie, zusätzlich zu den Unschärfemarkierern (,wahrscheinlich“, „so: bisschen“, „irgendwie“), mit ihrer nachgeschobenen Äußerung „keine AHnung“ (Z. 275) abschwächt, ihn somit weniger angreifbar macht und gleichzeitig indiziert, dass ihr weitere Deutungsalternativen aktuell nicht zugänglich sind. Jasmin nimmt dies mit einem „hm“ (Z. 276) zur Kenntnis. Damit wird die Bewertung der Lichteffekte von den beiden als abgeschlossen behandelt und im weiteren Gesprächsverlauf nicht noch einmal aufgegriffen. Auch in diesem Beispiel zeigt sich, dass an eine Erstbewertung nicht notwendigerweise unmittelbar eine Zweitbewertung angeschlossen werden muss, sondern erst nach Deutungen und erlebensdeklarativen Äußerungen (,am ANfang hab ich gedacht“) mit Bezug auf die Rezeption des Theaterstücks von einer Gesprächspartnerin eine Zweitbewertung des gleichen Bezugsobjekts vorgenommen wird, allerdings mit eingeschränkter Reichweite: Die Lichteffekte werden mit Bezug auf konkrete Stellen im Stück negativ, insgesamt (,an sich“) aber positiv bewertet.

In direktem Anschluss an Beispiel 78 fokussiert Annalena das Bühnenbild als Teilaspekt des Theaterstücks sowie als neues Bezugsobjekt auf der ästhetisch-performativen Ebene und konstatiert, dass sie das Bühnenbild ,auch intresSANT“ (Z. 277-280) fand:

\section{Beispiel 79: Siegen_14-10-26_Blut_Gr.2}

\begin{tabular}{|c|c|c|}
\hline 277 & Annalena: & ich fand_s auch intresSANT- \\
\hline 278 & & dass das BÜHnenbild ä: h ja: . \\
\hline 279 & & $(0.2)$ \\
\hline 280 & & sehr spoRAdisch gehalten [worden is-] \\
\hline 281 & Anna: & {$[j a:$. } \\
\hline 282 & Annalena: & ALso. $=$ \\
\hline 283 & & $=$ die hatten da ja fast GAR nich[ts? ] \\
\hline 8 & Anna: & {$[j a:-]$} \\
\hline
\end{tabular}


285

286

287

288

289

290

291

292

293

294 Anna:

295 Annalena:

296

297

298

299

300

301

302

303

304

305

306

307

308

309

310

311

312

313

314

315

316

317

318

319

320

321

322

323

Annalena:

Anna:

Jasmin:

Anna:
$(0.5)$

ja:-

(0.3)

das war (auch) GUT.

$(0.7)$

aber sowas MAG_ich eigentlich gerne.=

=weil du dich halt dann mehr auf das STÜCK öhkonzenTRIER[ST.

$[j$ ja geNAU. $=$ auf $]$

[DI:E-]

[j ] [a: .]

[auf] di : e äh KUNST de: r-

der SCHAUspieler.

[ja. ]

[ja.

(0.5)

${ }^{\circ} \mathrm{h}$ un_es war NICH so:-

$[(($ schmatzt $))]$

$[(j a)$.

(0.5)

Anna:

also nich so_n reaLIStisches theater-

dass man IRgendwi : e-

$(0.2)$

ja.

$(0.2)$

MANCHmal gedacht hat-

boah-

das passiert gerade !WIRK! lich was auf_a BÜHne issondern es WAR-

${ }^{\circ} \mathrm{h}$ SCHON so-

dass man die ganze zeit WUSSte-=

=oKE : -

es is_n theAterstück-

und so WEIter-

die sind da in ihren ROLlen und so-

${ }^{\circ} \mathrm{h}$ also (.) keine AHnung. =

=ich hab auch mal theAterstücke gesehen-

wo das-

${ }^{\circ} \mathrm{h}$ ! SO! rea!LIS! tisch geSPIELT wurde-

dass du irgendwie VOLL- 
324

325

326

327

328

329

330

331

332

333

334
$(0.8)$

also RICHtig da !DRIN! warst. =

=als wenn das gerade !WIRK! lich pasSIEren würde.=

=und NICHT nur irgendwie so_n the! A! terstück wär .

' $\mathrm{h}$ aber das fand ich da ja NICH. =

=also alLEIN schon durch diese-

ohh ä:h-

(0.4)

tz $\ddot{\mathrm{A}}: \mathrm{H}-$

LIEder da zwischendurch und so-

$(0.4)$

Annalena steigt in Zeile 277 mit einer selbstinitiierten Bewertung in die Bewertungssequenz ein, bei der sie die in den Zeilen 278 bis 280 vorgenommene Spezifizierung des Bewertungsgegenstands bereits durch die Proform „es“ projiziert. Wie auch Anna im vorangehenden Beispiel sichert sie durch die Wahl des nicht eindeutig negativ oder positiv konnotierten Adjektivs „interesSANT“ (Z. 277) ihre Positionierung gegen eine potenzielle (Face-)Bedrohung durch Nichtübereinstimmung ab. In direktem Anschluss spezifiziert Annalena dann das Bezugsobjekt ihrer Bewertung (,dass das BÜHnenbild ä:h ja:. (0.2) sehr spoRAdisch gehalten worden is“, Z. 278-280) mit ihrer resümierenden Feststellung „ALso. = = die hatten da ja fast GAR nichts?“ (Z. 282-283). Sowohl die prosodische Realisierung als auch die sequenzielle Positionierung der von Anna realisierten Rückmeldesignale (,,ja:“) in Zeile 281 und 284 in Überlappug mit Annalenas Äußerungen lassen darauf schließen, dass es sich um Zustimmung handelt (siehe Kapitel 2.3.1). Auch Jasmin schließt sich Annalenas Position an (Z. 286) und schreibt durch die ihrer Zustimmung angeschlossene positive Bewertung „das war (auch) GUT.“ (Z. 288) Annalenas Äußerung „ich fand_s auch intresSANT- dass das BÜHnenbild ä:h ja:. (0.2) sehr spoRAdisch gehalten worden is-“ (Z. 277-280) nachträglich den Status einer positiven Bewertung zu. Nach ihrem persönlichen Geschmacksurteil „aber sowas MAG_ich eigentlich gerne.“ (Z. 290) liefert sie in direktem Anschluss mit „= weil du dich halt dann mehr auf das STÜCK öh- konzen-TRIERST.“ (Z. 291-292) eine Begründung, die Annalena teilt (,ja geNAU“, Z. 293) und mit einem Wechsel des Bezugsobjekts vom Bühnenbild hin zur schauspielerischen Leistung und der Expansion „auf di:e äh KUNST de:r- der SCHAUspieler.“ (Z. 295-296) präzisiert. Anna erweitert den von Annalena gewechselten Fokus von der schauspielerischen Leistung auf die gesamte Inszenierung und projiziert im Rahmen dieser affektiv aufgeladenen Schilderung ein mögliches alternatives (Wahrnehmungs-)Szenario („,so_n reaLIStisches theater- dass man IRgendwi:e- (. . .) MANCHmal gedacht hat- boah- das passiert 
gerade !WIRK!lich was auf_a BÜHne is-“, Z. 304-311), um das gerade rezipierte Theaterstück als Gegenbeispiel davon abzugrenzen („॰h un_es war NICH so:-“, Z. 300; ,sondern es WAR- ${ }^{\circ} \mathrm{h}$ SCHON so- dass man die ganze zeit WUSSte- = = $\mathrm{oK}$ :E- es is_n theAterstück- und so WEIter- die sind da in ihren ROLlen und so-“, Z. 312-318). Mit der Referenz auf bisherige Rezeptionserfahrungen (,= ich hab auch mal theAterstücke gesehen- WO das- ${ }^{\circ} \mathrm{h}$ !SO! rea!LIS!tisch geSPIELT wurde- dass du irgendwie VOLL- (0.8) also RICHtig da !DRIN! warst. $==$ als wenn das gerade !WIRK!lich pasSIEren würde. $==$ und NICHT nur irgendwie so_n the!A!terstück wär.“, Z. 320-327) als Kontrastfolie vergleicht sie das im Rahmen des Theaterbesuchs Erlebte mit vergangenen Theaterbesuchen (siehe auch Beispiel 57, Kapitel 7.5), bevor sie wieder auf das gerade besuchte Theaterstück zurückkommt $\left({ }^{\circ} \mathrm{h}\right.$ aber das fand ich da ja NICH. $==$ also alLEIN schon durch diese- ${ }^{\circ}$ hh ä:h- (0.4) tz Ä:H- LIEder da zwischendurch und so-“, Z. 328-333). Auch in diesem Gesprächsausschnitt fällt auf, dass die Sprecherinnen ihre Bewertungen, Begründungen und Erlebensschilderungen durch sprachliche Mittel zum Indizieren von Vagheit unscharf markieren, sich so weniger angreifbar machen und einer eindeutigen Positionierung somit möglicherweise aus dem Weg gehen.

\subsubsection{Abgleich von Bewertungen durch ko-konstruierte Spezifizierungen des Bewertungsgegenstands}

Anders als in den vorangehend angeführten Ausschnitten, in denen eine Erstbewertung keine Zweitbewertung nach sich zieht, Zweitbewertungen zeitlich verzögert oder durch minimale Zustimmung realisiert werden, weist die Bewertungsinteraktion im nachfolgenden Beispiel die von Pomerantz (1984) sowie Auer und Uhmann (1982) herausgestellte sequenzielle Struktur auf (siehe Kapitel 2.3.1). In diesem Beispiel unterhalten sich Greta und Richard in der Pause des Theaterstücks Der Kaufmann von Venedig. Nachdem Greta und Richard sich mit einer Bekannten, die sie im Theaterfoyer treffen, über das Theaterstück unterhalten haben, referiert Richard in Zeile 153 auf Hintergrundwissen beziehungsweise bisherige Rezeptionserfahrungen hinsichtlich des Stücks. Als auch Greta ihren Bezug zum von ihnen rezipierten Theaterstück dargelegt hat, initiiert sie in Zeile 175 die Bewertungsinteraktion:

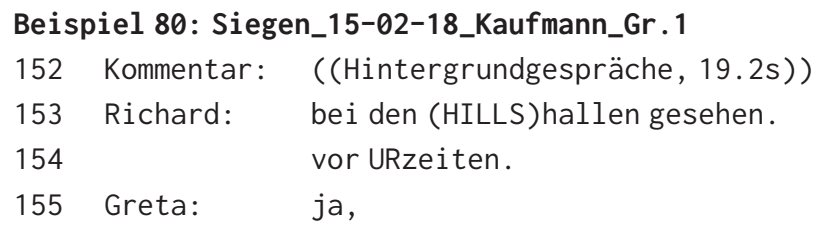


156

158 159

160

161

162

163

164

165

166

167 Richard:

168

169 Greta:

170

171

172 Richard:

173 Greta:

174

175

176

177

178

179 Richard:

180

181 Greta:

182

183

184

185 Richard:

186

187 Greta:

188

189 Richard:

190

191 Greta:

192 Richard:

193 Greta:

194
(2.6)

nee ich_hab mich (.) SCHON_auf eh in beZUG

auf_äh-

((Hintergrundgespräch))

(3.0)

das seminar über veNEdig-

(1.4)

und auch über die (. ) eh (. ) JÜdische präSENZ;

(0.3)

in veNEdig;

(0.6)

mit dem ersten GHETto un_[so weiter]=ne?

[ja_ja. ]

$(1.0)$

ä:h- (.)

(1.2)

kamen wir auf dieses-

ja_ja;

STÜCK;

$(0.5)$

und ich FINde (.) geRAde-

$(0.6)$

geRAde die figur des_äh- (. )

$(0.7)$

SHYlock;

(1.0)

SHYlocks des (.) JUden_is-

(0.3)

sehr schön geSPIELT=ne?

(1.5)

ja der-

$(0.3)$

ja;

(1.0)

die sind ALle gut=ne?

[aber der-]

[ja,

der ist nat[ürlich SPITze.]

[der ist- ]

der ist SPITze ja; 
Über den Verweis auf „das seminar über veNEdig“ (Z. 160) stellt Greta den Bezug zu einer im Stück agierenden Figur her (,und ich FINde (.) geRAde- (0.6) geRAde die figur des_äh-“, Z. 175-177), die von Richard nach einem Zögern Gretas projiziert und ko-konstruierend benannt wird („SHYlock;“, Z. 179). Greta vervollständigt daraufhin mit „SHYlocks des (.) JUden_is“ (Z. 181) ihre zuvor begonnene Äußerung und bewertet die schauspielerische Leistung der im Stück präsentierten Figur auf der ästhetisch-performativen Ebene explizit positiv (,sehr schön geSPIELT“, Z. 183). Mit dem ihrer Bewertung angeschlossenen Rückversicherungssignal fordert sie von Richard eine Bestätigung ihrer Bewertung ein, die Richard zumindest in Ansätzen liefert (Z. 185). In Zeile 189 erweitert Richard die Reichweite seiner im Vergleich zu Greta deskalierten Bewertung, indem er „ALle“ im Stück agierenden Figuren oder Schauspieler in den Fokus nimmt und mit „gut“ bewertet. Auch Richard fordert eine Bestätigung seiner Bewertung ein, fährt dann allerdings in Zeile 190, in Überlappung mit Gretas Bestätigung, mit einer Refokussierung der von Greta bewerteten Figur fort und hält mittels einer im Vergleich zu Gretas Erstbewertung eskalierten Bewertung der Figur fest, dass der die Figur verkörpernde Schauspieler beziehungsweise die dargestellte Figur „natürlich SPITze“ (Z. 192) sei. Nach Gretas Bestätigung durch das Aufgreifen von Richards Bewertung und einem zustimmenden „ja“ (Z. 193-194) behandeln die beiden die vorangehende Bewertungsinteraktion als abgeschlossen und widmen sich nach einer kurzen Pause der Getränkebestellung. Während eingangs des Ausschnitts Zustimmung vermehrt minimal (durch ,ja“ oder „genau“) verbalisiert wurde, zeigt die gemeinsam von Greta und Richard produzierte Bewertung einer im Stück agierenden Figur in ihrem sequenziellen Verlauf Merkmale der bereits in Kapitel 2.3.1 anhand interaktionslinguistischer Forschung beschriebenen Strukturen der Deskalierung und Eskalierung sowie Erstbewertung, Zweitbewertung und Zustimmung.

In den vorangehend angeführten Ausschnitten nehmen die Beteiligten mit ihren Bewertungen Teilaspekte der Inszenierung (unter anderem das Bühnenbild, im Stück agierende Figuren, die schauspielerische Performance, das Kostüm usw.) auf der ästhetisch-performativen Ebene oder die gesamte Inszenierung auf der globalen Ebene als Bezugsobjekte ihrer Bewertungen in den Fokus. Die Analysen der Beispiele zeigen, dass an (sowohl positive als auch negative) Bewertungen eine Deutungssequenz angeschlossen werden kann, in der sich die Beteiligten darüber verständigen, warum etwas gut oder schlecht war/ist und wie sie es interpretieren. Damit weisen die Verläufe in den präsentierten Ausschnitten mit dem Bewerten und daran angeschlossenen Deuten und Interpretieren drei der von Hausendorf für Gespräche vor Kunstwerken im Museum herausgestellten Aufgaben (siehe Kapitel 3.1) auf. Bewertungen können, so bleibt vorerst festzuhalten, als Einstiege in Deutungen dienen oder 
Deutungen abschließen, indem zum Beispiel das Ergebnis der Deutung als Bewertung formuliert wird (vgl. auch Linz 2018). Mit ihren zum Teil sehr emphatischen Ko-Konstruktionen von Bewertungsgegenständen, Bewertungen, Beschreibungen und Interpretationen, den oftmals in den vorangehend angeführten Beispielen zu beobachtenden Absicherungen gemeinsamer Wahrnehmungen, Deutungen und Interpretationen sowie der Offenlegung innerer, während der Rezeption erfahrener Erlebens- und Denkprozesse, um diese anderen am Gespräch Beteiligten zugänglich zu machen, tragen die Beteiligten zum geselligen Erlebnis des Theaterbesuchs (siehe Kapitel 4.1) sowie zur Vergemeinschaftung innerhalb der Gesprächsgruppe bei.

\subsubsection{Bewertungen mit Übertrag auf die Welt außerhalb des Theaterstücks}

Während in den in Kapitel 8.2.1 vorgestellten Gesprächsausschnitten von den Beteiligten vorwiegend Bezugsobjekte auf der inhaltlichen, ästhetischperformativen und globalen Ebene des Theaterstücks bewertet wurden, werden in den nachfolgenden Bewertungsinteraktionen im Theaterstück präsentierte Inhalte und/oder (Teil-)Aspekte auf die Ebene der eigenen Alltagswirklichkeit oder der Gesellschaft und somit auf die Welt außerhalb des Theaterstücks übertragen.

\subsubsection{Bewertungen als Anstoß persönlicher Erlebensschilderungen und Moralisierungen}

Im folgenden ersten Beispiel, in dem ein Übertrag der im Stück präsentierten Inhalte (vorerst) nur sehr vage angedeutet wird (Z. 053, 055 sowie 086-091), unterhalten sich Titus und Vanessa im Anschluss an das von ihnen besuchte Theaterstück Die Lücke (siehe auch Beispiel 62):

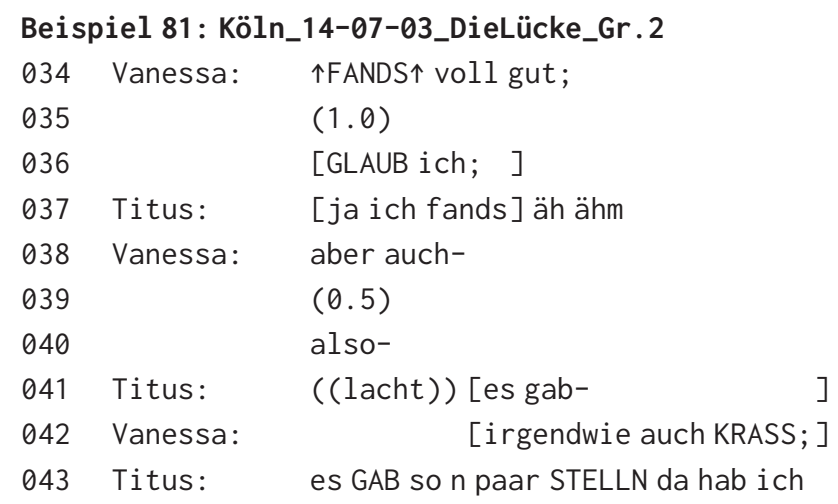




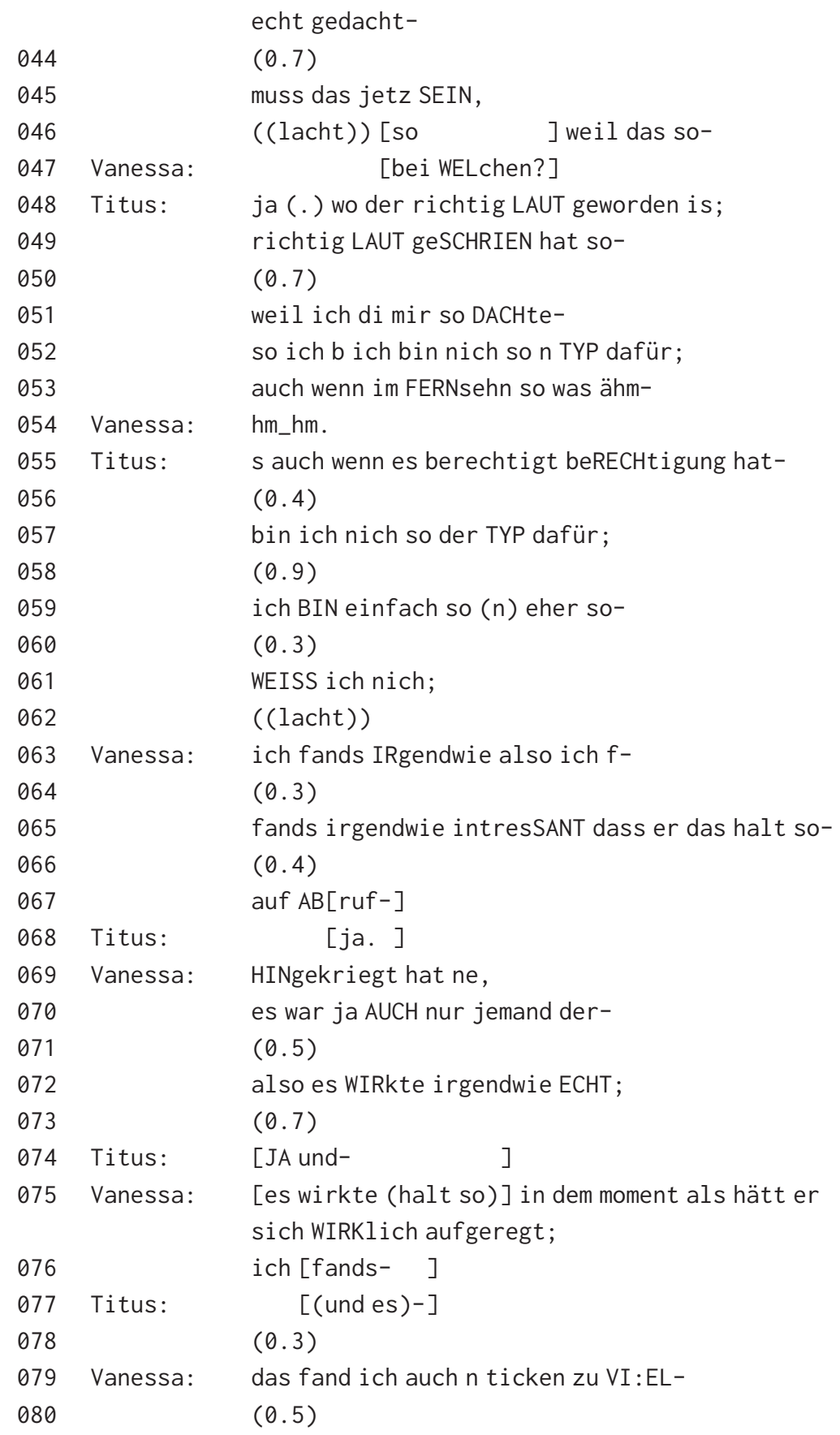


081

082

083 Titus:

084

085 Vanessa:

086 Titus: weil es sind ja diejenigen die WAR_N ja DA,

087 Vanessa: [ja. ]

088 Titus: [die ham] ja geLEBT zu der zeit;

089 desWE[gen; ]

090 Vanessa: $\quad[j a$.

091 Titus: $\quad$ irgendwie sin sind die ja mit involviert involVIERT gewesen;

092 Vanessa: ich finds $n$ bisschen ZU: ANgreifend glaub ich an die andern aber-

093

(0.5)

Vanessa gibt in Zeile 034 selbstinitiativ eine positive globale Erstbewertung ab, die sie nach einer kurzen Pause durch die Expansion „GLAUB ich“ (Z. 036) als unsicher rahmt (vgl. Imo 2006: 277) und als potentiell heikel und Facebedrohend markiert. In Überlappung mit Vanessas Äußerung scheint Titus eine ihrer Erstbewertung zustimmende Zweitbewertung äußern zu wollen (,ja ich fands äh ähm“, Z. 037), die er jedoch nicht weiter ausführt. Vanessa leitet in Zeile 038 eine scheinbare Fokusverschiebung ein (,aber auch“), die sie allerdings nur sehr zögerlich realisiert und vorerst nicht finalisiert. Titus versucht in Zeile 041 den Turn zu übernehmen, bricht seine Äußerung jedoch abermals ab, als Vanessa in Überlappung die Bewertung „irgendwie auch KRASS;“ (Z. 042) äußert. Hierbei bleibt allerdings offen, inwieweit (im positiven oder negativen Sinne) Vanessa etwas als krass empfunden hat, denn auch das Bezugsobjekt ihrer Bewertung wird nicht weiter konkretisiert. Somit umgeht diesmal Vanessa, wie auch schon Titus zu Beginn des Gesprächs (siehe Beispiel 62), eine eindeutige Positionierung und sichert sich gegen eine damit verbundene FaceBedrohung ab. Wurde von beiden Beteiligten mit der Proform „es“ im bisherigen Gesprächsverlauf in thematischer Hinsicht eher vage auf das Theaterstück beziehungsweise die Inszenierung in Gänze verwiesen, so fokussiert Titus ab Zeile 043 ebenfalls vage und vorsichtig „so n paar STELLN“ (siehe auch Beispiel 30, Kapitel 7.3), die er durch seine Erlebensdeklaration „da hab ich echt gedacht- (0.7) muss das jetz SEIN, “ implizit negativ bewertet. Nach der von Vanessa in Zeile 047 eingeforderten Konkretisierung des vagen Verweises auf „so n paar STELLN“ (Z. 043), liefert Titus mit Verweis auf die inhaltliche Ebene des Stücks eine Erläuterung zumindest einer der von ihm indizierten Stellen, nämlich 
„wo der richtig LAUT geworden is; richtig LAUT geSCHRIEN hat so-“ (Z. 048-049). Damit einhergehend findet - von Titus initiiert - ein Wechsel des Bezugsobjekts statt, bei dem nicht mehr die gesamte Inszenierung auf der globalen Ebene, sondern die Performance (das Schreien) einer Figur im Stück auf der ästhetischperformativen Ebene im Vordergrund steht. Seine implizit negative Bewertung in Zeile 043 bis 045 begründet Titus ab Zeile 051 im Anschluss an und mit Bezug auf den Verweis auf die von ihm angesprochene Stelle mit persönlichen Vorlieben (,weil ich di mir so DACHte- so ich b ich bin nich so $n$ TYP dafür; auch wenn im FERNsehn so was ähm- s auch wenn es berechtigt beRECHtigung hat- (0.4) bin ich nich so der TYP dafür;“). Nach dieser Begründung beginnt Titus mit „ich BIN einfach so (n) eher so-“ (Z. 059) scheinbar mit einer Selbstzuschreibung, die er nach einer Minimalpause und der wahrscheinlich im Zuge der Suche nach einer adäquaten Formulierung als Disfluenzmarker fungierenden Formel „WEISS ich nich;“103 (Z. 061) allerdings nicht zu Ende führt und mit einem Lachen abschließt. Ab Zeile 062 nimmt Vanessa eine Positionierung vor (,,ich fands IRgendwie also ich f- (0.3) fands irgendwie intresSANT dass er das halt so- (0.4) auf ABruf- HINgekriegt hat ne,“), die sie durch „irgendwie“ abschwächt und so als potenziell heikel markiert. Zudem umgeht sie mit der Wahl des Adjektivs „intresSANT“ als evaluativer Ausdruck eine eindeutige Positionierung. Mit ihrer Äußerung „also es WIRkte irgendwie ECHT; (0.7) es wirkte (halt so) in dem moment als hätt er sich WIRKlich aufgeregt;“ (Z. 072) verweist Vanessa auf den Authentizitätscharakter der schauspielerischen Performance und setzt in Zeile 076 mit „ich fands-“ scheinbar zu einer weiteren Bewertung an, die sich mit Titus’ Turnubernahme überlappt, und die Vanessa letztendlich erst nach kurzem Zögern vorsichtig und durch das eine unzweckmäßig hohe Ausprägung indizierende Adverb „zu“ negativ konnotiert ausführt („das fand ich auch $\mathrm{n}$ ticken zu VI:EL-“, Z. 079). Die in Vanessas Bewertung enthaltene Partikel ,auch“ deutet zum einen mit Blick auf Titus’ implizit negative Bewertung in Zeile 043-045 auf Vanessas Übereinstimmung mit Titus Position hin. Zum anderen gibt sie einen Hinweis darauf, dass Vanessa Titus' Erlebensdeklaration den Status einer Bewertung zuschreibt. Im Anschluss an ihre Bewertung setzt Vanessa zu einer Begründung an (,weil ich mir DACHte-“, Z. 081, siehe auch Beispiel 53, Kapitel 7.5), wird von Titus aber durch den Verweis „ich glaub der HAT sich auch wirklich aufgeregt;“ (Z. 083) und die daran angeschlossene Begründung „weil es sind ja diejenigen die WAR_N ja DA, die ham ja geLEBT zu der zeit; desWEgen; irgendwie sin sind die

103 Vergleiche zu weiß ich nicht als epistemische Unschärfeankündigung ausführlicher König (2014: 186-187). 
ja mit involviert involVIERT gewesen;“ (Z. 086-091) an weiteren Ausführungen gehindert. Mit ihrer unscharf markierten Äußerung ,ich finds n bisschen ZU: ANgreifend glaub ich an die andern aber-“ (Z. 092) verweist Vanessa vermutlich immer noch auf das gleiche Bezugsobjekt, nämlich das Schreien einer der im Stück agierenden Figuren, scheint jedoch ihre Bewertung auf eine moralische Ebene (,zu: ANgreifend“) zu übertragen und aus einer metareflexiven Perspektive auf die Inszenierung des Stücks zu argumentieren. Eine durch den Widerspruchsmarker „aber“ angedeutete Weiterführung ihrer Äußerung wird von Vanessa nicht realisiert, da Titus vorschlägt, sich im Foyer anders zu positionieren.

Dem folgenden Ausschnitt, der im weiteren Verlauf des Gesprächs zwischen Titus und Vanessa zu verorten ist, geht eine Gesprächspassage voran, in der sich Titus und Vanessa über das genaue Jahr der Ereignisse in der Keupstraße, die im Stück thematisiert werden, verständigen.

\section{Beispiel 82: Köln_14-07-03_DieLücke_Gr.2}

\begin{tabular}{|c|c|c|}
\hline $\begin{array}{l}179 \\
180\end{array}$ & Titus: & $\begin{array}{l}\text { ja also. } \\
(0.3)\end{array}$ \\
\hline 181 & & ((räuspert sich)) m war mir gar nich beWUSST- \\
\hline 182 & & ich dacht das wär noch LÄNger her. \\
\hline 183 & & $(0.3)$ \\
\hline 184 & & [gewesen; ] \\
\hline 185 & Vanessa: & [hm_hm? ] \\
\hline 186 & & $(0.9)$ \\
\hline 187 & Titus: & wie UNwissend; \\
\hline 188 & & e hehehe \\
\hline 189 & Vanessa: & $(($ lacht $))$ \\
\hline 190 & Titus: & von MIR; \\
\hline 191 & & $(0.5)$ \\
\hline 192 & Vanessa: & aber das fand [ich] EH ganz gut dass die so ähm- \\
\hline 193 & Titus: & {$[j a]$.} \\
\hline 194 & Vanessa: & wo du jetz GRAde wegen unwissen sagst ähm- \\
\hline 195 & Kommentar: & ((Klappern im Hintergrund)) \\
\hline 196 & Titus: & ja. \\
\hline 197 & & $(0.3)$ \\
\hline 198 & Vanessa: & dass es so AUFklärend war ne, \\
\hline 199 & & $(0.4)$ \\
\hline 200 & Titus: & auf jeden FALL ja; \\
\hline 201 & Kommentar: & ((Klappern im Hintergrund)) \\
\hline 202 & Vanessa: & also auch dass sie die FAKten nochmal so also \\
\hline
\end{tabular}




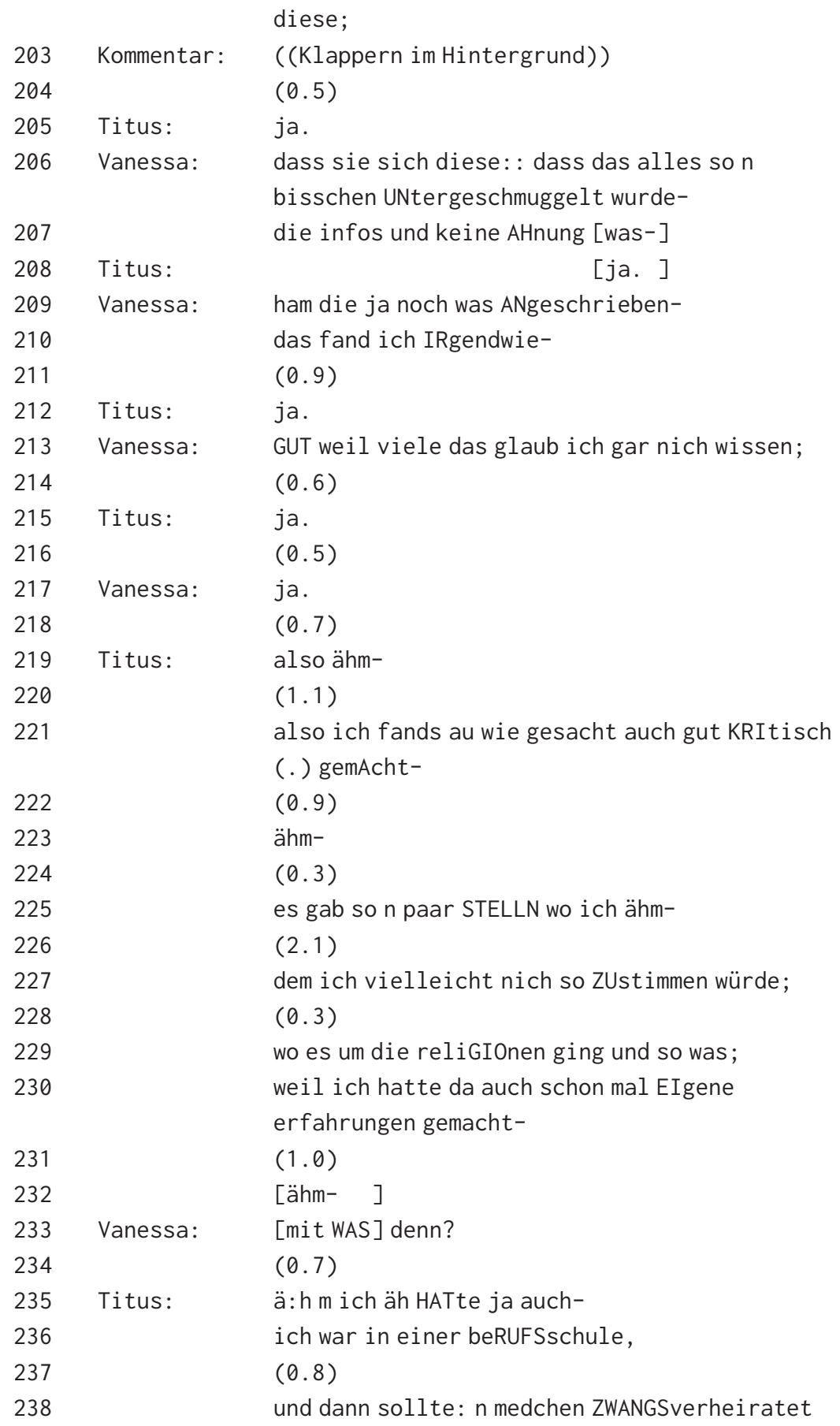


werden;

239

240

241 Vanessa:

242 Titus:

243

244

245

246

247

248

249

250

251

252

253

254

255

256

257

258
(0.2)

und wir hatten uns drum geKÜMmert;

das hast du glaub ich ma erZ̈̈HLT;

das hatt ich aber schonma erZ̈̈HLT;

(0.5)

also-

(0.3)

damit will ich nur SAgen dass ähm-

(0.8)

es GIBT dinge die äh-

$(0.5)$

die passiern halt SCHON;

$(0.4)$

und da muss man $\mathrm{SCHON}$ irgendwie gucken dass es irgendwie-

(0.7)

dass man da auch HILFT;

und dass man da auch irgendwie in irgendeiner

FORM ähm-

(1.0)

nich SAGT-

äh das geht mich nichts AN;

Von Zeile 192 bis 198 schließt Vanessa an Titus’ ironische Selbstkritik (,ja also. (0.3) ((räuspert sich)) m war mir gar nich beWUSST- ich dacht das wär noch LÄNger her. (0.3) gewesen; (( . .)) wie UNwissend; e hehehe ((. . )) von MIR;“ Z. 187-190) eine weitere, diesmal positive Bewertung an (,aber das fand ich EH ganz gut“), in der sie auf Titus’ Äußerung Bezug nimmt (,wo du jetz GRAde wegen unwissen sagst ähm“) und im Anschluss das Bezugsobjekt ihrer Bewertung nennt („dass es so AUFklärend war ne,“). Die durch Disfluenzmarker verzögerte und durch die unbetonte Gradpartikel „ganz“ abgeschwächte Bewertung schließt Vanessa mit dem Rückversicherungssignal „ne“ ab, fordert damit einerseits eine Bestätigung von Titus ein und spricht ihm andererseits so nachträglich die epistemische Autorität zu, die sie mit ihrer Erstbewertung beansprucht hat. Titus liefert die eingeforderte Bestätigung mit ,auf jeden FALL ja;“ (Z. 200) und bekräftigt somit Vanessas Erstbewertung. Vanessa expandiert daraufhin die Bewertungssequenz, indem sie sehr unsicher weitere vage Beschreibungen des Inszenierten $\mathrm{zu}$ formulieren beziehungsweise Beispiele $\mathrm{zu}$ finden versucht (,also auch dass sie die FAKten nochmal so also diese; dass sie 
sich diese:: dass das alles so $\mathrm{n}$ bisschen UNtergeschmuggelt wurde- die infos und keine AHnung was“, Z. 202-207). Die Abbrüche und Reformulierungen (,also auch dass sie die FAKten nochmal so also diese; dass sie sich diese::“), die Wortdehnung („diese::“) sowie das Markieren von Unschärfe (,so n bisschen so“) und der Ausdruck „UNtergeschmuggelt“ deuten auf Formulierungsprobleme beziehungsweise Schwierigkeiten bei der Suche nach einem Bezugsobjekt hin, das Vanessa letztendlich mit „das alles“ benennt und mit „die infos“ (Z. 207) zumindest teilweise konkretisiert. Die unmittelbar an ihre Äußerung mit dem Konnektor „und“ angeschlossene Formel „keine AHnung was“104 (Z. 207) fungiert in diesem Fall zum einen als Disfluenzmarker und indiziert zum anderen, dass Vanessa weitere (konkrete) Beispiele in diesem Moment nicht zugänglich sind. In Zeile 209 verweist Vanessa in unmittelbarem Anschluss, gemeinsames Hintergrundwissen unterstellend, mit „ham die ja noch was ANgeschrieben“ auf eine Stelle im Stück, bewertet diese nach einer Verzögerung (Z. 211) und Titus’ Hörrerrückmeldung (Z. 212) vorsichtig positiv („das fand ich IRgendwie- GUT“) und liefert eine Begründung ihrer Bewertung, indem sie aus der Perspektive der Rezipienten argumentiert („weil viele das glaub ich gar nich wissen;“, Z. 213). Nach einer weiteren Hörerrückmeldung von Titus (Z. 215) und einer kurzen Pause schließt Vanessa in Zeile 217 ihre Ausführungen mit einem „ja“ ab. Titus schließt durch seine globale Zweitbewertung der Inszenierung „also ich fands au wie gesacht auch gut KRItisch (.) gemAcht-“ (Z. 221) an Vanessas Beitrag an, verweist im Anschluss daran jedoch wiederholt auf „so $n$ paar STELLN“ (Z. 225), denen er „vielleicht nich so ZUstimmen würde“ (Z. 227). Den vagen Verweis „so n paar STELLN“ konkretisiert Titus dann unaufgefordert in Zeile 229 („wo es um die reliGIOnen ging und so was;“) und begründet seine Bewertung mit der Andeutung, bereits persönliche Erfahrungen gemacht zu haben („weil ich hatte da auch schon mal EIgene erfahrungen gemacht-“, Z. 230, siehe auch Beispiel 54, Kapitel 7.5). Wie in Ausschnitt 81 fordert auch hier Vanessa in Zeile 233 eine Konkretisierung von Titus ein. Titus berichtet daraufhin von der von ihm angedeuteten Erfahrung, wechselt auf die Ebene der gesellschaftlichen Wirklichkeit und argumentiert ab Zeile 246 verallgemeinernd („man“) aus einer moralischen Perspektive („es GIBT dinge die äh- (0.5) die passiern halt SCHON; (0.4) und da muss man SCHON irgendwie gucken dass es irgendwie- (0.7) dass man da auch HILFT; und dass man da auch irgendwie in irgendeiner FORM ähm- (1.0) nich SAGT- äh das geht mich nichts AN;“). Der moralisierende Appell wird von Titus durch „irgendwie gucken dass es irgendwie“

104 Vergleiche zu keine Ahnung als epistemische Unschärfeankündigung König (2014: 190-191). 
und „irgendwie in irgendeiner FORM“ abgeschwächt. Zudem distanziert er sich durch das betonte „SCHON“ implizit von denjenigen, die sagen, „das geht mich nichts AN“ (Z. 258). Anhand dieses Beispiels wird deutlich, wie sowohl Vanessa als auch Titus, angestoßen durch Bewertungen auf der inhaltlichen Ebene des Theaterstücks, das im Theaterstück Rezipierte auf die Ebene ihrer eigenen Alltagswirklichkeit beziehungsweise der gesellschaftlichen Wirklichkeit übertragen und schließlich - eine allgemeine Perspektive einnehmend darüber moralisieren. Titus' scherzhafter und selbstironischer Umgang mit dem Aufdecken seines Nicht-Wissens durch Vanessa zu Beginn des Ausschnitts gibt einen Hinweis auf die damit verbundene Gefahr der Blamage (siehe Kapitel 3.1). Dass dieses Nichtwissen von Vanessa allerdings nicht dazu genutzt wird, sich in irgendeiner Weise von Titus zu distanzieren, sondern dass sie Titus' Verweis aufgreift, um eine Stärke der Inszenierung herauszustellen und um darauf hinzuweisen, dass möglicherweise auch anderen Rezipienten das Hintergrundwissen fehlt, hebt den kooperativen Charakter der Gespräche hervor (siehe Kapitel 4).

Eine ähnliche Dynamik ist auch im folgenden Gespräch zwischen Susanne und Dina zu erkennen, das ebenfalls im Anschluss an das Theaterstück Die Lücke stattfindet. Die im Stück präsentierten Inhalte werden im Anschluss an eine Bewertung und eine Selbstreflexion von den Gesprächspartnerinnen auf ihre eigene Alltagswirklichkeit übertragen und es wird aus einer allgemeinen Perspektive moralisiert:

Beispiel 83: Köln_14-09-25_DieLücke_Gr.3

$\begin{array}{lll}001 & \text { Susanne: } & \left(\left(\text { schmatzt)) alles KLAR. }{ }^{\circ} \mathrm{h}\right.\right. \\ 002 & & (2.7) \\ 003 & \text { Dina: } & (\text { ich fand das) S0 GRUselig }(.) \text { teilweise es war } \\ & & \text { richtig KRASS. } \\ & \text { ja. } \\ 004 & \text { Susanne: } & (0.5) \\ 005 & & \text { als sie da mit der KAmera standen. } \\ 006 & \text { Dina: } & \text { oa: } \text { h }^{\circ} \\ 007 & \text { Susanne: } & (1.1) \\ 008 & & \text { s WAHNinnig gut gemacht. } \\ 009 & \text { Dina: } & (2.1) \\ 010 & & \text { also ECHT gut. } \\ 011 & & (2.3) \\ 012 & & \text { weil es is ja irgendwie so_n THEma mit dem man sich } \\ 013 & & \text { ja norMAlerweise NICH so beschäftigt. } \\ & \end{array}$


015

016

017

018 Susanne:

019

020

021

022

023

024

025

026

027

028

029 Dina:

030 Susanne:

031

032 Dina:

034

035

036

037 weil es STIMMT ja.

man denkt ja von sich selber dass man toleRANT ist.

(1.8)

ja ich beschäftige mich SCHON damit weil ich ja

JEden tag in der ARbeit auch damit zu tun hab eigentlich.

(1.1)

ja:-

$(0.3)$

ja KLAR $<<$ lachend $>$ ich $>-$

Susanne: also über dies[e GANze- ]

Dina: [bei MIR gibt_s] ja eigent

[lich keine AUSländer in der ((unverständlich))]

Susanne: [diese GANze all

tags (.) rasSISmus geschichte und was macht des

mit_nem KIND des da-

$(0.8)$

äh

$(0.4)$

[jaja naTüRlich. ]

[äh un das ist jeden] tag erLEBT und so.= =also des is ja wirklich was wo ich mich JEden tag mit beSCHÄFtige un wo ich auch viel mit den stuDIErenden drüber spreche und [so aber-]

[naTÜRli ]ch aber

nich mit (. ) dem rasSISmus der möglicherweise von DIR ausgeht.

$(0.6)$

weil man denkt ja WIRKlich von sich selber NEIN ich ich BIN nich rassistisch.

ich bin super toleRANT.

ich bin OFfen.

$(0.5)$

Unmittelbar zu Beginn des Gesprächs initiiert Dina mit „(ich fand das) SO GRUselig (.) teilweise es war richtig KRASS.“ (Z. 003) die Bewertungsinteraktion. Vorerst offen bleibt hierbei allerdings das konkrete Bezugsobjekt von Dinas Bewertung sowie ob die Bewertung negativ oder positiv ist, und damit auch eine eindeutige Positionierung Dinas. Susanne fordert keine Konkretisierung ein, 
sondern stimmt Dinas Bewertung mit „ja“ (Z. 004) zu. Daran anschließend spezifiziert Dina auf der inhaltlichen Ebene zumindest die Szene im Theaterstück (,als sie da mit der KAmera standen.“, Z. 006), auf die sie referiert, was Susanne mit der affektiv aufgeladenen Vokalisation „oa::h“ (Z. 007) quittiert. In den Zeilen 009 und 011 bewertet Dina vermutlich die Inszenierung mit „s WAHNsinnig gut gemacht“ explizit positiv und verleiht ihrer positiven Bewertung mit „also ECHT gut.“ nochmals Nachdruck, wobei das Bezugsobjekt und damit auch die Reichweite der Bewertung nicht eindeutig spezifiziert werden. Ab Zeile 013 folgt eine als Begründung formulierte Selbstreflexion Dinas (,weil es is ja irgendwie so_n THEma mit dem man sich ja norMAlerweise NICH so beschäftigt.“), in der sie allgemein eine mangelnde Reflexion hinsichtlich der Thematik unterstellt, von der sich Susanne allerdings distanziert (,,ja ich beschäftige mich SCHON damit“, Z. 018). Ihre Nichtübereinstimmung mit Dinas pauschalisierender Äußerung begründet Susanne mit einem abgeschwächten Übertrag des im Theaterstück präsentierten und von Dina thematisierten Inhalts auf ihre (berufliche) Alltagswirklichkeit (,weil ich ja JEden tag in der ARbeit auch damit zu tun hab eigentlich.“, Z. 018). Während auch Dina die Situation auf ihren Alltag bezieht (,bei MIR gibt_s ja eigentlich keine AUSländer in der ((unverständlich))“, Z. 024), expandiert Susanne ihre Erläuterung von Zeile 025 bis 030 (,diese GANze alltags (.) rasSISmus geschichte und was macht des mit_nem KIND des da- (0.8) äh (0.39) äh un das ist jeden tag erLEBT und so.") und betont nochmals, dass sie sich täglich mit dieser Thematik auseinandersetzt (,, = also des is ja wirklich was wo ich mich JEden tag mit beSCHÄFtige un wo ich auch viel mit den stuDIErenden drüber spreche und so aber-“, Z. 031). Dina quittiert die Äußerungen Susannes mit dem Selbstverständlichkeitsmarker ${ }^{105}$ „naTÜRlich“ (Z. 032), schränkt ihre Zustimmung dann allerdings mit einem Verweis auf mangelnde Selbstreflexion ein (,,aber nich mit (.) dem rasSISmus der möglicherweise von DIR ausgeht.“), und argumentiert ab Zeile 034 auf einer allgemeineren, moralischen Ebene, indem sie vermutlich zwar aus ihrer Perspektive, aber verallgemeinernd („man“) formuliert ihren Gedankengang verbalisiert (,weil man denkt ja WIRKlich von sich selber NEIN ich ich BIN nich rassistisch. ich bin super toleRANT. ich bin OFfen. “).

Im weiteren Gesprächsverlauf wird das Thema, nachdem Dina und Susanne sich mit Getränken versorgt haben, wieder aufgegriffen. Es kommt zu einer weiteren Bewertung und damit einhergehend auch zu einem Moralisieren über den Umgang mit Schülern mit Migrationshintergrund in Dinas Schulalltag:

105 Vergleiche König (2014: 241) zur kritisierenden Verwendungsweise von natürlich. 


\section{Beispiel 84: Köln_14-09-25_DieLücke_Gr.3}

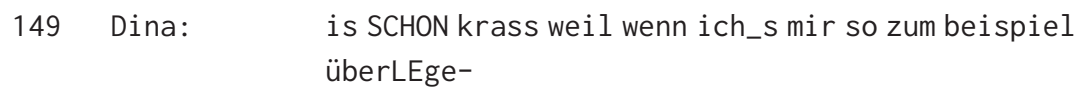

$150 \quad(1.1)$

151 Susanne: [ja.]

152 Dina: [oK ]AY es is jetz natürlich auch-

$153 \quad$ (2.9)

154 Susanne: $\quad(($ schmatzt $))($ re $)-$

$155 \quad(2.3)$

156 Dina: is natürlich auch beDENKlich die schule auf die_m-

$157 \quad(1.1)$

158 wir GEHN und so aber-

$159 \quad(0.4)$

160 wenn man sich_s mal so überLEGT-

161 EIgentlich hab ich echt äh (.) KAUM kontakt mit AUSländern.

(1.1)

Susanne: ich KENN WEnig leute mit migraTIONShintergrund. in meiner KLASse gibt es-

Dina:

(0.7)

ja.

(0.4)

EIne von der man WIRKlich sagen könnte dass sie noch IRgendwie migrationshintergrund HAT .

un DIE wird-

(0.4)

RICHtig rassistisch angemacht von meiner ENglischlehrerin. die sarojini kommt aus INdien.

176 Susanne: $(0.6)$

Dina:

hmhm? INdischen akzent gewÖHnen (is doch)-

182 Susanne: s_allerLETZte $j_{1}[a . \quad]$ 
183 Dina:

184

185
Susanne:
${ }_{1}[$ ich HÖ]re KEInen indischen akZENT. $<$ lachend $>$ ich ${ }_{2}\left[\right.$ DENke $\left.>-h^{\circ}\right]$

${ }_{2}$ [ja es is ] abSURD.

In Zeile 149 schließt Dina mit ihrer Bewertung „ist SCHON krass“ an ein bereits von ihr im vorangehenden Gesprächsverlauf angesprochenes Thema an (siehe Beispiel 83, Z. 024). In direktem Anschluss beginnt Dina mit einer Begründung (,weil“), die sie allerdings abbricht und stattdessen nach einer kurzen Pause mit einer vagen Bewertung fortfährt, die sich wahrscheinlich auf eine Situation an ihrer Schule bezieht (,,is natürlich auch beDENKlich die schule auf die_m(1.1) wir GEHN und so aber-“, Z. 152-158). Während Dina in Zeile 149 noch aus ihrer subjektiven Perspektive spricht, wechselt sie im Zuge ihres Verweises auf ihre Schule in die erste Person Plural „wir“ und wechselt dann, vermutlich selbstreflektierend, auf eine allgemeine (Meta-)Ebene („man“, Z. 160), bevor sie in Zeile 161 mit Bezug zu ihrer Alltagswirklichkeit wieder aus ihrer eigenen, subjektiven Perspektive berichtet („EIgentlich hab ich echt äh (.) KAUM kontakt mit AUSländern. (1.1) ich KENN WEnig leute mit migraTIONShintergrund.“). Die anschließende Schilderung der situativen Umstände ihres (damaligen) Erlebens ${ }^{106}$ nutzt Dina als Emotionalisierungstechnik, um bereits implizit eine Bewertung $\mathrm{zu}$ indizieren. Sie greift exemplarisch eine Schülerin aus ihrer Klasse heraus (,in meiner KLASse gibt es- (0.7) ((. . .)) (0.4) EIne von der man WIRKlich sagen könnte dass sie noch IRgendwie migrationshintergrund HAT.“, Z. 164-168), schildert deren Hintergrund („die sarojini kommt aus Indien.“, Z. 174) und eine Situation aus dem Unterrichtsgeschehen (,un DIE wird- (0.4) RICHtig rassistisch angemacht von meiner Englischlehrerin.“, Z. 170-172), die sie durch den von ihr gewählten lexikalischen Ausdruck (,angemacht“) ebenfalls negativ auflädt. Im Rahmen der anschließenden, affektiv aufgeladenen Reinszenierung der Lehrerin („ICH als ameriKAnerin kann mich einfach nich an dein INdischen akzent geWÖHnen“, Z. 181, siehe auch Beispiel 44, Kapitel 7.4) bewertet Dina mithilfe paraverbaler Mittel die Situation negativ und setzt dann mit „(is doch)“ in Zeile 181 scheinbar zu einer expliziten Bewertung an, die von Susanne in direktem Anschluss in Ko-Produktion mit den Worten „s_allerLETZte ja.“ (Z. 182) vervollständigt wird. Diese Finalisierung der Bewertung durch Susanne parallel zu der noch nicht abgeschlossenen Bewertung Dinas ist ein Beispiel für die Orientierung der in die Bewertungsaktivität involvierten Sprecher an der sich im Vollzug der Interaktion entfaltenden Struktur von

106 Vergleiche ausführlicher Fiehler (2014: 46) zur Beschreibung und Erzählung der situativen Umstände eines Erlebens. 
Sprecheräußerungen als „projected social actions“ (Lindström/Mondada 2009a: 300, Goodwin 2018; siehe auch Kapitel 2.3.1). Dina liefert in den Zeilen 183 und 184 weitere Erläuterungen (,ich HÖre KEInen indischen akZENT. <<lachend> ich DENke>- ${ }^{\circ}$ “), die sie aber abbricht und nicht wieder aufgreift. Susanne merkt in Überlappung mit Dinas Äußerung in Zeile 184 an, dass die von Dina geschilderte Situation „abSURD“ (Z. 185) sei. Der ihrer Bewertung vorangestellte Zustimmungsmarker „ja“ (Z. 185) weist darauf hin, dass Susanne Dinas Reinszenierung ihrer Lehrerin den Status einer Bewertung zuschreibt.

Auch in den beiden vorangehend analysierten Beispielen 83 und 84 mündet die durch eine Bewertung der Inszenierung initiierte Bewertungsinteraktion schließlich in einem Übertrag der im Stück präsentierten Thematik auf persönliche Erfahrungen der beiden Gesprächspartnerinnen und in weitere Bewertungen der von ihnen in ihren Erfahrungen geschilderten gesellschaftlichen Verhältnisse.

\subsubsection{Bewertungen als Anstoß von Projektionen auf die Ebene der gesellschaftlichen Realität}

Im folgenden Beispiel nutzen die am Gespräch Beteiligten Annalena, Anna, Jasmin und Pascal ihre Bewertung der im Stück Verrücktes Blut inszenierten Lehrerrolle als Anstoß zu einem Übertrag auf die Alltagswirklichkeit:

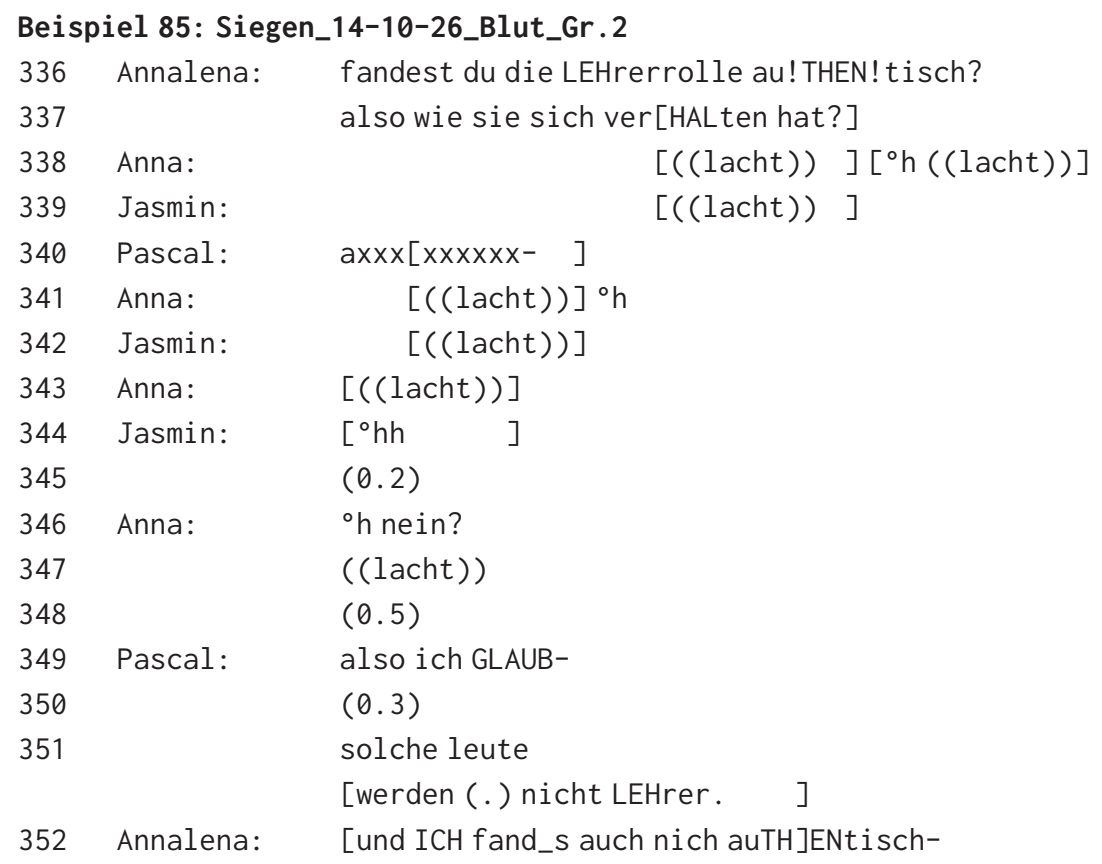




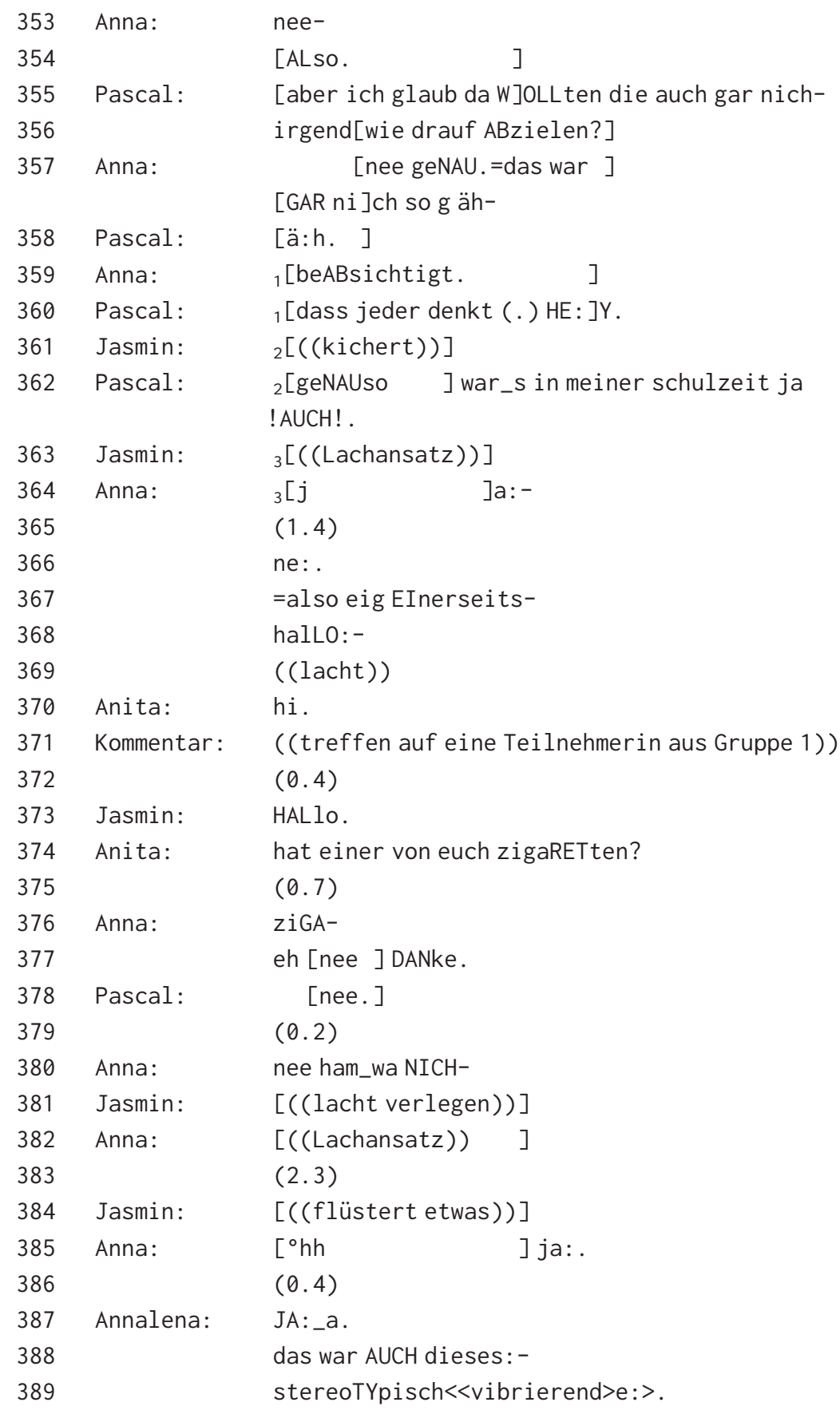


390

391

392

393

394

395

396

397

398

399

400

401 Annalena:

402 Jasmin:

403 Anna:

404 Pascal:

405 Jasmin:

406

407

408 Annalena:

409 Pascal:

410

411 ((lacht)) unfähige $<<$ lachend $>$ LEHrerbild $>$ ?

ja:

$(0.4)$

mh-

AH_oh .

$(0.5)$

mh_JOA.

$\mathrm{hh}^{\circ}$

ich (GLAUB) di :e-

frau hatte eigentlich schon LÄNGST $n$ burn! OUT! gehabt.

und [(wär halt ]

$[<<$ lachend $>$ geNAU $>$. ]

${ }_{1}[\mathrm{n}$ LEben $<<$ lachend $>$ also $>$ ) -$]$

${ }_{1}[<<$ lachend $>$ ja: $>$.

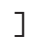

(wär) schon (. ) längst im URlau[b s. ]

[überhaup]t nicht

DURCHse[tzungsfä ]hig?

$(0.2)$

ja: .

Nachdem Annalena ihre Gesprächspartnerin Anna explizit zu einer Stellungnahme aufgefordert und so die Bewertungsinteraktion initiiert hat, Anna die relevant gesetzte Stellungnahme geliefert und Pascal ebenfalls eine Vermutung geäußert hat, nimmt auch Annalena selbst zu ihrer Frage Stellung (siehe dazu ausführlicher Beispiel 68). Ab Zeile 355 äußern sowohl Pascal als auch Anna vorsichtig ihre Vermutung, dass eine authentische Darstellung der im Stück agierenden Lehrerin gar nicht beabsichtigt war (,aber ich glaub da WOLLten die auch gar nich- irgendwie drauf ABzielen? ((. . .)) dass jeder denkt (.) HE:Y. geNAUso war_s in meiner schulzeit ja !AUCH!.“, Z. 355-362). Nachdem die Beteiligten kurz mit einer ihnen bekannten anderen Theaterbesucherin interagiert haben, greift Annalena das vorher bearbeitete Thema wieder auf und bewertet die Inszenierung der Lehrerfigur im Stück (,das war AUCH dieses:stereoTYpisch<<vibrierend>e: >. ((lacht)) unfähige <<lachend>LEHrerbild >?“, Z. 388-390). Im Anschluss an die minimale Zustimmung der anderen Gesprächteilnehmer (Z. 391-396) projiziert Jasmin mit „ich (GLAUB) di:e- frau hatte eigent- 
lich schon LÄNGST n burn!OUT! gehabt. und (wär halt $n$ LEben <<lachend > also >)((. . .) $)^{\circ}$ h (1.1) (wär) schon (.) längst im URlaub s.“ (Z. 398-407) die im Stück inszenierte Lehrerfigur in die Alltagswelt außerhalb des Theaterstücks (siehe auch Beispiel 56, Kapitel 7.5). Anna, Annalena und Pascal stimmen Jasmins Äußerungen zu (Z. 401-404), bevor Annalena festhält, dass eine Lehrerin, wie sie im Stück präsentiert wurde, in der Realität ,überhaupt nicht DURCHsetzungsfähig“ (Z. 408) wäre, was wiederum von Pascal durch ein Lachen (Z. 409) und von Anna durch ein zustimmendes ,ja:“ (Z. 411) quittiert wird.

Im Anschluss an die gemeinsame Bewertung der im Stück verkörperten Lehrerin initiiert Jasmin mit ihrer negativen Bewertung der Inszenierung der Schulklasse im Theaterstück in Zeile 413 eine weitere Bewertungssequenz (,also ich fand die KLASse auch !ZU! extrem dargestellt-“), mit der sie vom zuvor thematisierten Lehrerbild und der Projektion auf die Ebene des Stücks wechselt:

\section{Beispiel 86: Siegen_14-10-26_Blut_Gr.2}

\begin{tabular}{|c|c|c|}
\hline 412 & & $(2.7)$ \\
\hline 413 & Jasmin: & $\begin{array}{l}\text { also ich fand die KLASse auch ! ZU! extrem } \\
\text { dargestellt- }\end{array}$ \\
\hline 14 & & [am ANfang \\
\hline 15 & Pascal: & [ja es es waren!SEHR! ] VIE[le: . ] \\
\hline 6 & Jasmin: & [und WO. ] \\
\hline 7 & & $(0.4)$ \\
\hline 18 & & {$[$ wo- $]$} \\
\hline 19 & Pascal: & $\begin{array}{l}\text { [also] eigentlich! AL!le (.) VORurteile die du } \\
\text { ha[ben] [kannst.] }\end{array}$ \\
\hline 20 & Jasmin: & {$[j a-]$} \\
\hline 21 & Anna: & {$[j a: . \quad]$} \\
\hline 422 & & $(0.3)$ \\
\hline 423 & & ${ }^{\circ} \mathrm{h}$ aber wie geSAGT. = \\
\hline 424 & & $=$ ich find die hatten auch so ihre ROLlen. \\
\hline 425 & & also das is auch OFT [so- \\
\hline 426 & Pascal: & {$[($ immer $)][$ WENN dann- $]$} \\
\hline 427 & Anna: & {$\left[{ }^{\circ} \mathrm{h}\right.$} \\
\hline 28 & Pascal: & ge[NAU. $]$ \\
\hline 29 & Anna: & [man ] weiß ja GAR nich- \\
\hline & & wie die dann (.) tatSÄCHlich sind- \\
\hline & & aber so ! IN! ihrer ROLle? \\
\hline & & sind die dann halt SO und so- \\
\hline
\end{tabular}




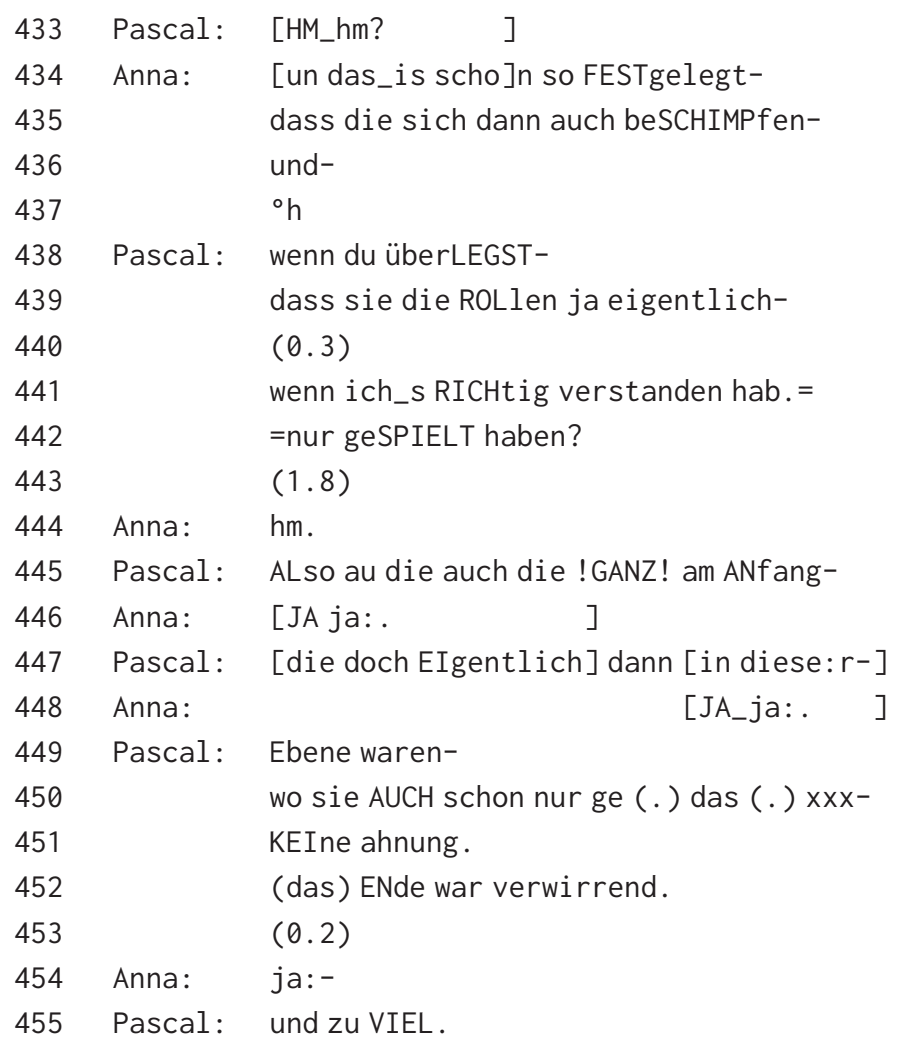

Die Reichweite ihrer Bewertung schränkt Jasmin in unmittelbarem Anschluss mit dem Verweis „am ANfang.“ (Z. 414) nachträglich ein. Pascal schließt sich Jasmins Bewertung an und führt die Art der Darstellung (,ja es es waren !SEHR! VIEle:. (. . .) also eigentlich !AL!le (.) VORurteile die du haben kannst.“, Z. 415 und 419) als Begründung der negativen Bewertung an. In Zeile 424 kontrastiert Anna durch den Verweis darauf, dass die im Stück agierenden Darsteller Rollen einnehmen (,=ich find die hatten auch so ihre ROLlen.“), die im Stück verkörperten Personen sowie deren Verhalten mit den hinter den verkörperten Personen stehenden Schauspielern, von denen „man ja GAR nich“ weiß, „wie die dann (.) tatSÄCHlich sind-“ (Z. 429-430). Anschließend betont Anna nochmals, dass die im Stück präsentierten Figuren „!IN! ihrer ROLle (. . .) dann halt SO und so“ agieren (Z. 431) und ihr Verhalten bereits im Vorhinein und von außen bestimmt ist (,un das_is schon so FESTgelegt- dass die sich dann auch beSCHIMPfen-“, Z. 434-435). Angestoßen durch Annas Differenzierung verschiedener Realitätsebenen (gespielte Rollen vs. reale Personen) (vgl. Linz 2018: 230) setzt Pascal in Zeile 438 zu einem Deutungsversuch an, bricht diesen aber in Zeile 451 mit dem 
Unschärfemarker „KEIne ahnung“ ab (siehe auch Beispiel 34, Kapitel 7.3), der darauf hinweist, dass Pascal weitere Deutungsmöglichkeiten nicht zugänglich sind beziehungsweise er mit einer Deutung überfordert ist. Dies bestätigt auch seine anschließende Prädikation „(das) ENde war verwirrend. und zu VIEL.“ (Z. 452/ 455), mit der er das Ende des Theaterstücks in den Fokus nimmt, mittels des eine unzweckmäßig hohe Ausprägung indizierenden Adverbs „zu“ (siehe Kapitel 7.1 und 7.4) negativ bewertet, und mit dieser Bewertung die Bewertungssequenz der dargestellten Klasse als abgeschlossen behandelt. Anhand der beiden Ausschnitte aus dem Gespräch zwischen Anna, Annalena, Jasmin und Pascal wird deutlich, wie Bewertungen im Theaterstück präsentierter Inhalte Projektionen auf die Ebene der gesellschaftlichen Realität anstoßen und als Einstieg in Deutungen dienen sowie als Ergebnis aus diesen hervorgehen können.

\subsubsection{Bewertungen als Anstoß der Reflexion von Entwicklungstendenzen gesellschaftlicher Verhältnisse}

Im nachfolgenden Ausschnitt, ${ }^{107}$ der einem Gespräch zwischen Viktoria und Donata im Anschluss an das Theaterstück „Brain and Beauty“ entstammt, übertragen die beiden Freundinnen ebenfalls ihnen im Stück präsentierte Inhalte auf ihre eigene Alltagwirklichkeit sowie auf die gesellschaftliche Ebene. Viktoria initiiert die Bewertungsinteraktion, indem sie das „Thema“ des Theaterstücks in abgeschwächter Form („n bisschen“) negativ bewertet:

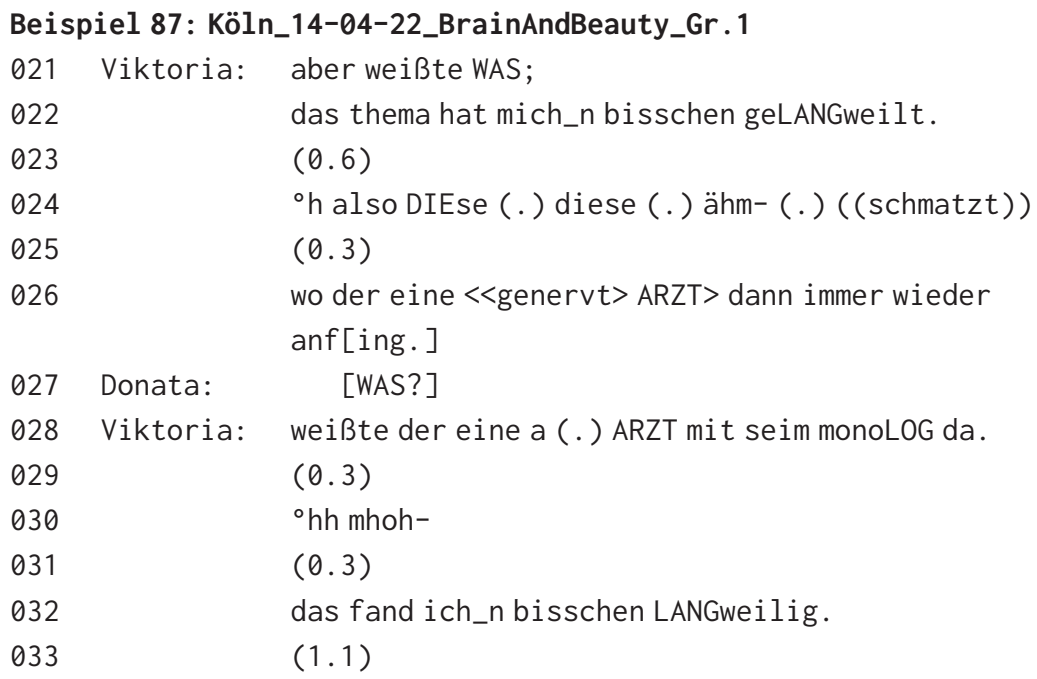

107 Siehe zu einer früheren Analyse dieses Beispiels Hrncal (2018: 258-260). 
$\begin{array}{lll}034 & \text { Donata: } & \text { ja aber so blaSIERT sind sie nun mal. } \\ 035 & \text { Viktoria: } & \text { ja }<<\text { lachend }>\text { wahrSCHEINlich>. } \\ 036 & \text { Donata: } & ((\text { lacht })) \\ 037 & & (7.2)\end{array}$

Eine sofortige Reaktion Donatas auf Viktorias Erstbewertung bleibt aus. Nach einer kurzen Pause projiziert Viktoria in Zeile 024 dann, eingeleitet durch das Adverb „also“ Erläuterungen, die sie nachfolgend $\mathrm{zu}$ formulieren versucht. An der Wiederholung des Demonstrativpronomens „DIEse“ beziehungsweise „dieser“ in attributiver Stellung, den Pausen und dem Verzögerungssignal „ähm“ wird deutlich, dass eine präzise Formulierung Viktoria zunächst Schwierigkeiten bereitet. Nach kurzem Zögern bietet Viktoria dann mit dem vagen Verweis auf die Stelle, ,wo der eine <<genervt>ARZT> dann immer wieder anfing.“ (Z. 026) einen Konkretisierungsvorschlag an, mit dem sie gleichzeitig eine Fokusverschiebung vom übergreifenden Thema des Stücks zu einer im Stück agierenden Figur auf der inhaltlichen Ebene vornimmt. Zusätzlich lädt Viktoria durch die Art und Weise der prosodischen Konturierung ihren Verweis auf die Figur im Stück mit negativer Wertung auf. Kurz bevor Viktoria ihre Äußerung abschließt, setzt Donata in Zeile 027 mit ihrer Nachfrage eine Reaktion Viktorias relevant, die Viktoria mit der spezifizierenden Expansion „weißte der eine a (.) ARZT mit seim monoLOG da.“ (Z. 028) liefert. Nach dem Affektmarker „mhoh“ in Zeile 030 greift sie mit der Proform „das“ als Bewertungsgegenstand ihre vorangehenden Äußerungen wieder auf und rahmt diese mit der vorsichtig formulierten Bewertung „das fand ich_n bisschen LANGweilig“ als abgeschlossen. Konkretisierungen hinsichtlich des von ihr angesprochenen Themas sowie der Figur im Stück und auch hinsichtlich der Reichweite ihrer abschließenden Bewertung nimmt Viktoria nicht vor. Auch Donata fordert keine weiteren Konkretisierungen ein, sondern bewertet mit ihrer Viktoria nur partiell zustimmenden Äußerung ,ja aber so blaSIERT sind sie nun mal.“ (Z. 034) entweder die im Stück präsentierten Ärzte oder Schönheitschirurgen im Allgemeinen negativ. Viktoria quittiert diese partiellen Widerspruch (,aber“) indizierende Bewertung Donatas mit einem lachend realisierten „wahrSCHEINlich“ (Z. 035), woraufhin Donata ebenfalls zu lachen beginnt. Die Bewertungssequenz der Ärzte (sowohl im Stück als auch bezogen auf die Gesellschaft) wird im Weiteren von keiner der beiden Sprecherinnen expandiert, sondern von ihnen als abgeschlossen behandelt. Die vorliegende Bewertungssequenz bestätigt die von Pomerantz (1984) sowie von Auer und Uhmann (1982) herausgestellte mögliche sequenzielle Struktur von Bewertungsinteraktionen: Viktorias Erstbewertung setzt eine Zweitbewertung konditionell relevant, die Donata nach Viktorias Spezifizierungsversuch des Bezugsobjekts ihrer Bewertung auch liefert. Die allerdings nur partiell zustimmende Zweitbewer- 
tung Donatas wird von Viktoria in einem dritten Schritt in abgeschwächter Form (, <<lachend > wahrSCHEINlich>“) bestätigt.

Nach einer Gesprächspause von etwa sieben Sekunden greift Viktoria die in der Inszenierung als Teil des Bühnenbilds aufgestellten Schaufensterpuppen, ${ }^{108}$ die sie auf dem Weg vom Theatersaal ins Theaterfoyer auf der Bühne sieht, als Gesprächsthema auf:

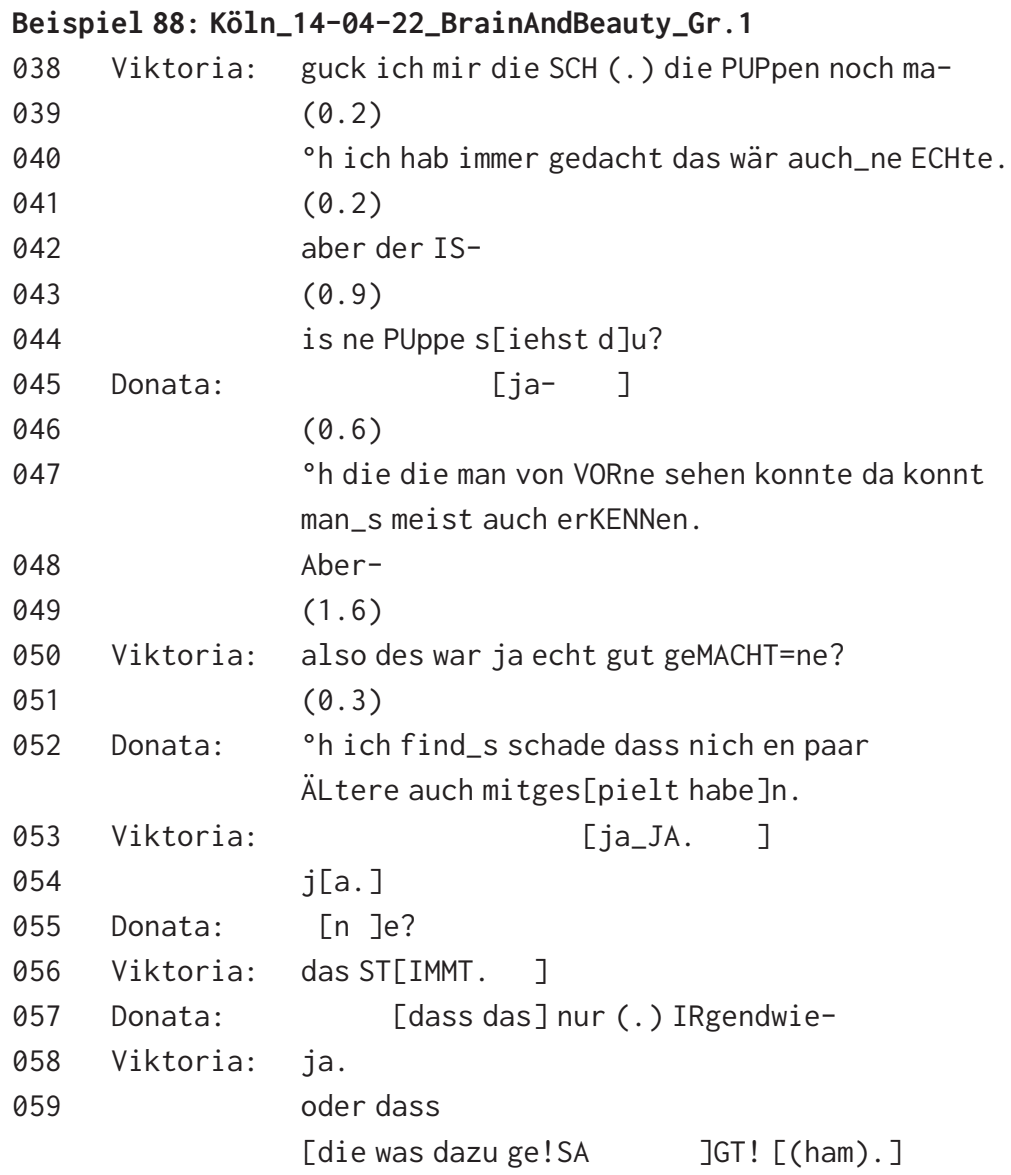

108 Neben den Schaufensterpuppen stehen als weiterer Teil des Bühnenbilds auch Schauspieler auf der Bühne, die sich allerdings während des gesamten Stücks nicht bewegen. Vom Zuschauerraum aus ist teilweise nicht zu erkennen, ob es sich um eine Schaufensterpuppe oder einen Schaupieler handelt (siehe auch Kapitel 6.6). 


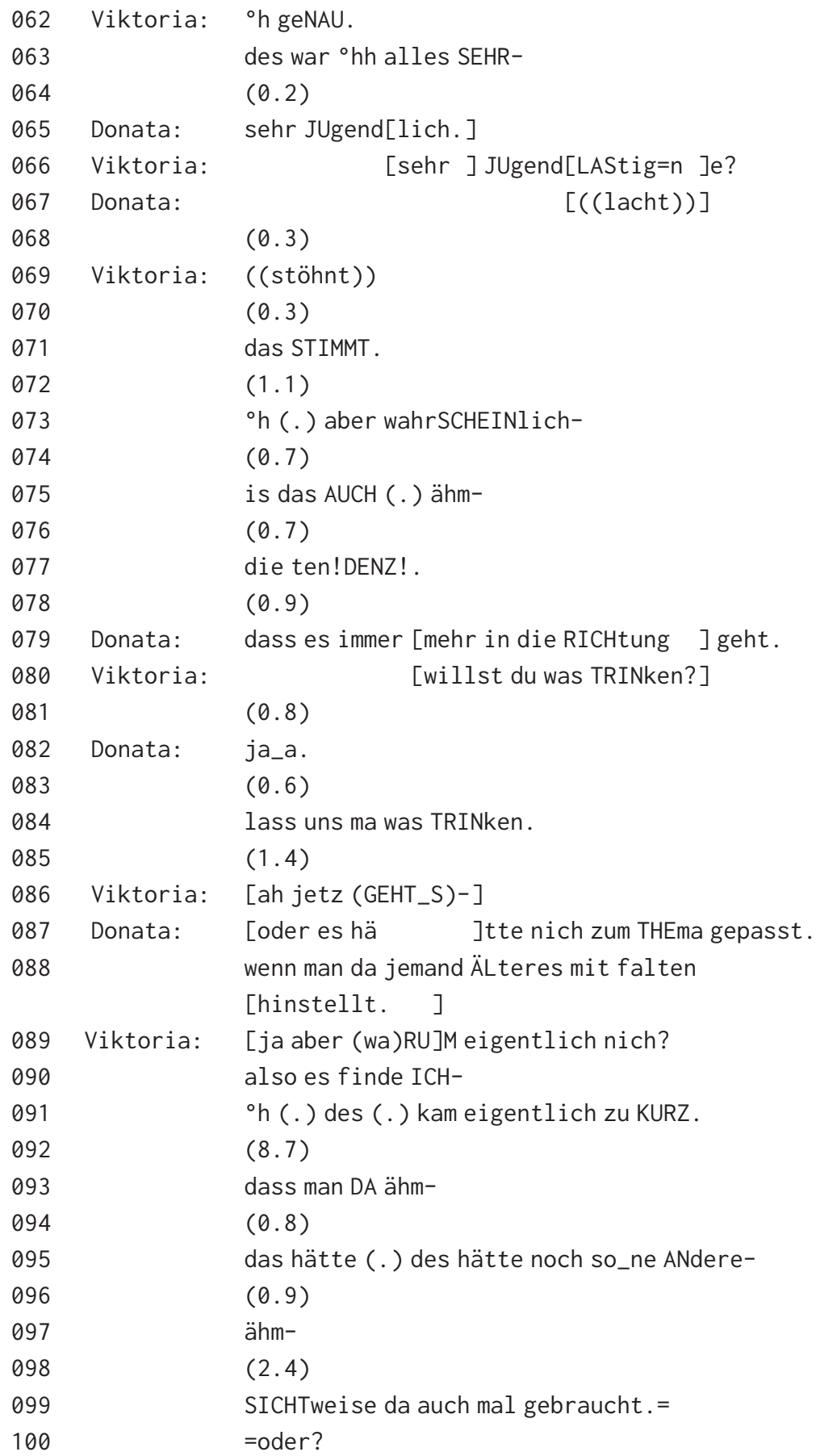


101

102

103

104

105

106

107

108

109

110

111

112

113

114 Donata:

115

116

117 Viktoria:

118

119

120

121

122

Donata:
(4.2)

Viktoria: $\quad h^{\circ}$ (a_weißte) was ich ja (.) interessant fand.

diese (.) äh GOLdenen ! STRUMPF! hosen=ne?

$<<$ lachend $>$ dies [das sah ] TOLL aus $>((\text { lacht }))^{\circ} \mathrm{h}$ gedacht.

$[(($ lacht $))]$

Viktoria: ${ }^{\circ} \mathrm{hh}$

Donata: ja aber nich nur STRUMPFhosen;

auch an den [ARmen.

]

Viktoria: [auch an den AR]men.

Donata: ja_ja die hatte so_n GANZkörperanzug.

$(0.8)$

Viktoria: ich nehm jetz en WEISSwein.

(3.1)

un wenn dann oben der ha (. ) ECHte HALS rauskam sah der-

(0.3)

direkt HÄSSlich $[<<$ lachend $>$ aus $>$. ((lacht $))]$

[ja (. ) STIMMT.

$(0.3)$

${ }^{\circ} \mathrm{hh}$

$(0.9)$

${ }^{\circ} \mathrm{h}$

(3.1)

In Zeile 040 verweist Viktoria lokal sensitiv mit „ ${ }^{\circ} \mathrm{h}$ ich hab immer gedacht das wär auch_ne ECHte.“ auf eine bestimmte Puppe, die sich aktuell im Wahrnehmungsraum der beiden Gesprächsteilnehmerinnen befindet, und stellt fest, dass es sich tatsächlich um eine Puppe handelt (,aber der IS - (0.9) is ne PUppe“). Sie fordert Donata mit einem unmittelbar angeschlossenen „siehst du?"“ zu einer Bestätigung und damit Absicherung ihrer Wahrnehmung auf, die Donata in Überlappung (Z. 045) auch liefert. Donata hält fest, dass die Puppen, „die man von VORne sehen konnte“ (Z. 047) meistens auch als Puppen identifizierbar waren (,da konnt man_s meist auch erKENNen.“). In Zeile 048 scheint sie mit „Aber-“ ihre Äußerung einschränken zu wollen, bricht dies jedoch ab. Nach einer kurzen Pause in Zeile 050 realisiert Viktoria mit „also des war ja echt gut geMACHT ne?“ eine Allgemeingültigkeitsanspruch erhebende positive Bewertung, ohne das Bezugsobjekt, auf das sie mit der Proform „des“ referiert, zu konkretisieren. Mit dem ihrer Bewertung angeschlossenen Rückversicherungssignal „ne?“ lädt sie Donata zum einen zu einer Zustimmung ein, zum 
anderen schreibt sie Donata, die durch die Rezeption des Theaterstücks den gleichen Zugang zum Bewertungsgegenstand hat, die von ihr mit ihrer Erstbewertung beanspruchte epistemische Autorität zu. Eine Zustimmung Donatas bleibt allerdings aus. Stattdessen kritisiert Donata die Unterrepräsentiertheit einer bestimmten Altersgruppe („ ${ }^{\circ} \mathrm{h}$ ich find_s schade dass nich en paar ÄLtere auch mitgespielt haben.“, Z. 052), verweist so implizit auch auf ihre Erwartungen an das Theaterstück und nimmt damit einen Teilaspekt der Inszenierung in den Fokus. Viktoria stimmt Donatas Einwand zu, noch bevor Donata ihre Äußerung abgeschlossen hat (Z. 053). Trotz der bereits zweifach von Viktoria gelieferten Zustimmung fordert Donata in Überlappung mit Viktorias „,ja“ durch das Rückversicherungssignal „ne?“ (Z. 055) nochmals eine Bestätigung ihrer Kritik von Viktoria ein, die diese in Zeile 056 auch liefert. Ab Zeile 057 kommt es zu einer ko-produzierten, quasi simultanen Spezifizierung der Kritik Donatas, die den beiden Sprecherinnen - ähnlich wie in Beispiel 80 - zugleich als Abgleich ihrer Standpunkte und zur gegenseitigen Versicherung ihrer Übereinstimmung dient (,ja.“, Z. 061; „'h geNAU.“, Z. 062). In Zeile 063 setzt Viktoria mit „des war ${ }^{\circ h}$ alles SEHR-“ zu einer erneuten Bewertung an, die sie allerdings abbricht und die Donata antizipierend (vgl. Lerner 1996) zu Ende führt („sehr JUgendlich.“, Z. 065), während Viktoria ihre Bewertung ebenfalls abschließt und eine Bestätigung Donatas einfordert („sehr JUgendLAStig = ne?“, Z. 066). Donata vervollständigt Viktorias Bewertung durch die Gradpartikel „sehr“ und das eigentlich beschreibende, allerdings affektiv mit Wertung aufgeladene Adjektiv „JUgendlich“ (Z. 065) noch bevor Viktoria diese finalisiert hat. Dies spricht auch in diesem Fall dafür, dass Donata die Struktur der Äußerung von Viktoria („des war `hh alles SEHR“, Z. 063) bereits als Projektion einer Bewertung identifiziert hat (vgl. Goodwin/Goodwin 1992 sowie Goodwin 2018). Die divergierenden Bewertungen „JUgendlich“ versus „JUgendLAStig“ quittiert Donata mit Lachen (Z. 067), Viktoria in Zeile 071 mit der Bestätigung „das STIMMT“. Nach dieser Kritik eines Teilaspekts der Inszenierung überträgt Viktoria das auf der Ebene des Stücks Präsentierte auf die Welt außerhalb des Theaterstücks und resümiert kontrastierend mit Blick auf die Gesellschaft, dass dies „wahrSCHEINlich (. . .) die ten!DENZ!“ (Z. 073-077) sei. Dass die Bearbeitung des Themas von Viktoria mit ihrer Äußerung als abgeschlossen behandelt wird, zeigt sich an ihrer mit Donatas Expansion „dass es immer mehr in die RICHtung geht.“ (Z. 079) überlappenden Frage „willst du was TRINken?“ (Z. 080), mit der sie zu einem neuen Thema, das für die beiden aktuell relevant wird, überleitet und damit gleichzeitig einen Ausstieg aus der auf Kunst im weiteren Sinne fokussierten Kommunikation initiiert. Donata bejaht die Frage, fährt dann aber mit einer Expansion des vor Viktorias Nachfrage bearbeiteten Themas fort und versucht, aus einer metareflexiven Perspektive mögliche 
Gründe der Betonung der Jugend in der Inszenierung zu finden („oder es hätte nich zum THEma gepasst. wenn man da jemand ÄLteres mit falten hinstellt.“, Z. 087-088). Viktoria geht auf Donatas Initiierung ein und hinterfragt die von Donata angeführten Gründe (,,ja aber (wa)RUM eigentlich nich?“, Z. 089), bevor sie festhält, dass „des (...) eigentlich zu KURZ“ kam.“ (Z. 090-091). Das von ihr durch die Proform „es“/,des“ vage gehaltene Bezugsobjekt versucht sie im Anschluss an ihre negative Bewertung zu konkretisieren (,das hätte (.) des hätte noch so_ne ANdere- (0.9) ähm- (2.4) SICHTweise da auch mal gebraucht.“, Z. 095-099) und sich durch ein nachgeschobenes Rückversicherungssignal („oder?“, Z. 100) bei Donata hinsichtlich einer gemeinsamen Positionierung abzusichern. Die durch Viktoria konditionell relevant gesetzte Reaktion von Donata bleibt allerdings aus, und die Themenbearbeitung gerät ins Stocken. Nach einer längeren Pause löst Viktoria das Problem, indem sie einen Themenwechsel von der metareflexiven auf die ästhetisch-performative Ebene des Theaterstücks vollzieht und das Kostüm einer im Stück dargestellten Figur als beiden Gesprächspartnerinnen zugängliches Bezugsobjekt in den Fokus nimmt (Z. 102-103) und so Donata den Wiedereinstieg ermöglicht. Im Anschluss an Viktorias explizit positive Bewertung (Z. 104) nimmt Donata eine Spezifizierung des Bezugsobjekts (Kostüm der Schauspielerin) vor (Z. 107-108). Viktoria bestätigt - teilweise in Überlappung mit Donata - deren Einwand. Donata präzisiert die Beschreibung des Kostüms der Schauspielerin mit ,ja_ja die hatte so_n GANZkörperanzug“ (Z. 110). In Zeile 112 nimmt Viktoria erneut einen Themenwechsel (,ich nehm jetz en WEISSwein.“) vor, während Donata an ihre Beschreibung mit einer negativen Bewertung mit Bezug zur Schauspielerin anschließt (,un wenn dann oben der ha (.) ECHte HALS rauskam sah der- (0.3) direkt HÄSSlich <<lachend>aus>.“, Z. 114-116). Mit Viktorias in Überlappung geäußerter Zustimmung (,,ja (.) STIMMT“, Z. 117) wird die Bewertung der Schauspielerin beziehungsweise ihres Kostüms als abgeschlossen gerahmt. Auch in diesem Ausschnitt findet im Rahmen der Suche nach Gründen für die Art und Weise der Inszenierung und der Nicht-Beteiligung einer bestimmten Altersgruppe ein Übertrag auf die gesellschaftliche Ebene beziehungsweise eine reflektierende Metaebene statt. Viktorias Strategie, dem Schweigen ihrer Gesprächspartnerin (Z. 101) mit einem Themenwechsel auf die ästhetischperformative Ebene des Theaterstücks zu begegnen, gibt einerseits einen Hinweis darauf, dass die Aushandlung der Bewertung von Teilaspekten des Theaterstücks (wie hier des Kostüms) für die Beteiligten weniger heikel und diesen besser zugänglich ist, als beispielsweise die Suche nach Gründen für die Art und Weise der Inszenierung auf der gesellschaftlichen beziehungsweise auf einer metareflexiven Ebene. Andererseits weist Viktorias Versuch, Donata (wieder) am 
Gespräch zu beteiligen, auf den kooperativen Charakter der Pausengespräche als geselliges Ereignis hin, und lässt darauf schließen, dass zumindest innerhalb der Gesprächsgruppe keine soziale Distinktion anvisiert wird.

\subsubsection{Ergebnisse}

Die Antwort auf die eingangs von Kapitel 8.2 aufgeworfene Frage, wie die an den Gesprächen Beteiligten Bewertungen in sequenzieller Hinsicht gemeinsam produzieren und aushandeln und inwiefern Bewertungen auf der Ebene des Theaterstücks dazu genutzt werden, um über gesellschaftliche Zusammenhänge zu moralisieren, wird auf Basis der in den Kapiteln 8.2.1 und 8.2.2 durchgeführten Analysen im Folgenden skizziert.

\section{Ko-Produktion von Bewertungen}

Mit Blick auf Erstbewertungen fällt auf, dass die Beteiligten vor allem Bewertungsgegenstände auf der ästhetisch-performativen Ebene des Theaterstücks in den Fokus nehmen oder eine Globalbewertung des Theaterstücks beziehungsweise der Inszenierung (,es“) äußern. Letztere Beobachtung spricht für die Feststellung Kindts (2007: 57), dass Gespräche über Kunst oftmals mit einer Pauschalbewertung begonnen werden. Eine Konkretisierung des Bezugsobjekts wird entweder gar nicht, nach dem Ausbleiben einer Reaktion des Gegenübers durch den Erstbewertenden selbst oder durch das Gegenüber in Form einer Zweitbewertung vorgenommen. In einigen Fällen wird eine Konkretisierung des Bezugsobjekts explizit vom Rezipienten durch eine Nachfrage eingefordert. Dies muss aber nicht notwendigerweise der Fall sein, denn in vielen Fällen folgt auf eine Erstbewertung als zweiter Schritt minimale Zustimmung, die durch „ja“, „das stimmt“ oder „genau“ kommuniziert wird. Eine Reaktion des Gegenübers kann des Weiteren gänzlich ausbleiben, was entweder zu einer Wiederholung der Erstbewertung durch den gleichen Sprecher führt oder zu einer erneuten Bewertung, mit der ein anderes Bezugsobjekt auf der gleichen Ebene (zumeist auf der ästhetisch-performativen Ebene) oder einer anderen (zumeist von der globalen Ebene hin zur ästhetisch-performativen) Ebene in den Fokus genommen wird. Eine weitere Möglichkeit ist, dass auf eine Erstbewertung zwar eine zweite Bewertung des Gegenübers folgt, allerdings hinsichtlich eines anderen Bezugsobjekts entweder auf der gleichen oder einer anderen Ebene. Somit sind die in der interaktionslinguistischen Forschung anhand privater Alltagsgespräche herausgestellten sequenziellen Verläufe von Bewertungen (vgl. unter anderem Auer/Uhmann 1982, Pomerantz 1984) in den in dieser 
Arbeit angeführten Beispielen selten zu beobachten und können um die oben genannten Verläufe mit spezifischem Bezug zum semi-institutionellen Kontext der Theaterpausengespräche ergänzt werden.

\section{Herstellen eines gemeinsamen Hintergrunds}

Hinsichtlich der in Kapitel 3 diskutierten Frage, inwiefern die Beteiligten während der Rezeption überhaupt in einen wie unter anderem von Krotz (1997) konstatierten inneren Dialog mit sich selbst treten, geben die in den vorangehend analysierten Ausschnitten von den Beteiligten verbalisierten Erlebensschilderungen einen Hinweis. Die Flüchtigkeit der im Theaterstück rezipierten Inhalte sowie die durch die Institutionsspezifik des Theaters bedingte Restriktion, dass eine Unterhaltung parallel zum Rezeptionsprozess nicht angemessen ist (siehe Kapitel 2.3.2), ziehen - ähnlich wie dies beim Sprechen über Fernsehinhalte der Fall ist (siehe Kapitel 3.1) - nach sich, dass im Vorfeld der Realisierung einer Bewertung oftmals in unterschiedlichem Maße kommunikativer Mehraufwand betrieben werden muss, um den Bewertungsgegenstand $\mathrm{zu}$ rekonstruieren und diesen so allen Gesprächsteilnehmern zugänglich zu machen. Im Falle von Erlebensschilderungen kann diese Rekonstruktion einerseits in Form einer Verständigung beider Gesprächspartner über ihr Erleben während der Rezeption des Theaterstücks stattfinden oder andererseits indem einer der beteiligten Sprecher sein inneres Erleben mit Bezug auf bestimmte im Stück präsentierte Inhalte schildert. Die Beteiligten lassen die jeweils anderen Gesprächsteilnehmer so an ihrer Wahrnehmung und ihren Denkprozessen, die sie auch explizit als solche rahmen, teilhaben, schaffen so einen gemeinsamen Hintergrund für weitere Bewertungen, Beschreibungen oder Deutungen und tragen auf diese Weise zu einem inkludierenden Charakter der Pausengespräche bei.

\section{Absichern gegen potenzielle Face-Bedrohungen}

Dass trotz des inkludierenden Charakters die von den Beteiligten selbstinitiiert oder durch Aufforderungen realisierten Erstbewertungen in großen Teilen von Vorsichtsmaßnahmen begleitet werden, zeugt von der Gefahr, in die sich die Beteiligten mit ihren Positionierungen begeben (müssen). Durch Subjektivierungen und Unschärfemarkierungen werden Bewertungen abgeschwächt und so als potenziell heikel und Face-bedrohend markiert oder die Beteiligten vermeiden eine Konkretisierung des Bewertungsgegenstands, indem sie nur vage durch eine Proform auf das Bezugsobjekt ihrer Bewertung verweisen. Die Vagheit der Bewertungen (siehe auch Kapitel 7.3) äußert sich allerdings nicht nur in der Art des Verweises auf das Bezugsobjekt selbst, sondern unter anderem auch durch eine nachträgliche Abschwächung der ganzen Bewertung durch 
Unschärfemarkierer wie glaub ich, weiß nich oder keine Ahnung. Dies gilt sowohl für negative als auch für positive Erstbewertungen. Durch die Wahl entsprechender evaluativer Ausdrücke wie „krass“ oder „interessant“, die nicht eindeutig positiv oder negativ konnotiert sind, umgehen sie schließlich den Zugzwang, sich eindeutig hinsichtlich eines bestimmten Bewertungsgegenstands positionieren zu müssen.

\section{Reflexion der eigenen Lebenswelt}

Im Falle eines Übertrags im Stück präsentierter Inhalte auf die gesellschaftliche Ebene außerhalb des Stücks schließt sich an eine Bewertung auf der Ebene des Theaterstücks zumeist erst ein Bezug zu eigenen Erfahrungen beziehungsweise zur eigenen Lebenswelt an, bevor ein Bezug zur gesellschaftlichen Realität vorgenommen wird. Die Beispiele zeigen auch, wie Bewertungen den Betiligten als Anstoß zur Selbsreflexion oder zur Projektion im Stück agierender Figuren und deren Positionen in die Realität dienen. Durch diesen Übertrag des Rezipierten auf die eigene Lebenswelt und die gesellschaftliche Wirklichkeit reflektieren sie ihre Rolle in der Gesellschaft und eignen sich die ihnen im Theaterstück präsentierten Inhalte explizit an (vgl. Faber 2001: 34; siehe auch Kapitel 3.1).

\subsection{Aushandlung von Nichtübereinstimmung in Bewertungsinteraktionen}

In konversationsanalytischen Studien zu Bewertungsinteraktionen in privaten Alltagsgesprächen wurde die Präferenz für Übereinstimmung als ein grundlegendes konversationelles Prinzip herausgestellt (siehe Kapitel 2.3.1). Die in Kapitel 8.1 und 8.2 analysierten Ausschnitte lassen aufgrund der Realisierung von Bewertungen darauf schließen, dass auch in den Bewertungsinteraktionen in Gesprächen in der Theaterpause eine Präferenz für Übereinstimmung beziehungsweise für übereinstimmende Zweitbewertungen vorliegt. Dafür sprechen nicht nur unmittelbar an eine Bewertung angeschlossene Nachfragen wie „findste nich auch“ oder Rückversicherungssignale (ne/gell), sondern auch - wie in der Ergebnisdarstellung in Kapitel 8.2.3 bereits zusammengefasst - der Einsatz von Hedging sowie eine nachträgliche Subjektivierung der Bewertung, Disfluenzmarker und Unschärfemarkierungen (keine Ahnung, weiß nicht), mit denen die Sprecher ihre Bewertung und die damit verbundene Positionierung als potenziell heikel markieren. Trotz der erkennbaren und von den Beteiligten indizierten Präferenz für Übereinstimmung wurde deutlich, dass Zweitbewertungen auch als nur partiell zustimmend oder nicht übereinstimmend realisiert werden können. 
Von Nichtübereinstimmung ist im Folgenden die Rede, wenn eine gegenläufige Zweitbewertung eines Gesprächspartners als Reaktion auf die Erstbewertung des anderen Gesprächspartners hinsichtlich eines Bewertungsobjekts vorliegt, wenn also im weiteren Sinne ,eine Äußerung sich gegen eine vorherige Äußerung eines anderen Diskussionspartners richtet“ (Gruber 1996: 168, Herv. im Original). Diese Nichtübereinstimmung kann von den Beteiligten ohne explizite lexikalische Widerspruchsmarker ${ }^{109}$ geäußert werden, und manifestiert sich auf der paraverbalen Ebene, wie dies im folgenden Ausschnitt in Marcos Zweitbewertung der Fall ist:

Beispiel 89: Siegen_14-11-14_Hamlet_Gr.2

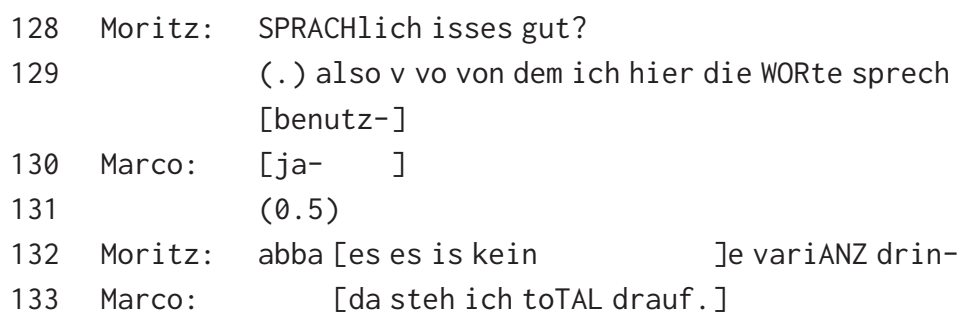

Eine weitere Möglichkeit ist das Realisieren von Nichtübereinstimmung unter der Verwendung äußerungsinitialer ${ }^{110}$ oder äußerungsinterner expliziter Widerspruchsmarker wie nein/nee/nö, (ja) aber/schon/doch oder stimmt nicht (vgl. auch Gruber 1993, 1996). Im folgenden Beispiel indiziert Nelli direkten Widerspruch mithilfe lexikalischer Widerspruchsmarker:

\section{Beispiel 90: Köln_14-06-15_Kaufmann_Gr.2}

273 Nelli: ich FINde die beiden:-

274 die d die (.) ZWILlinge-

275 oder die die ZWILlinge.

$276 \quad$ (.) ö:h-

$278 \quad(0.9)$

279 also WIE die [das-]

280 Wanja: [ja: ]

[aber (. ) das waren doch MEHR oder weniger-]

281 Nelli: [mh wie die das MAchen:?

282 Wanja: das-

109 Den Terminus ,Widerspruchsmarker übernehme ich von Gruber (1996).

110 Die Termini ,äußerungsinitial‘ und, äußerungsintern` übernehme ich ebenfalls von Gruber (1996). 
283

284

285

286

287

288

289

290

291

292

293

294

295

296

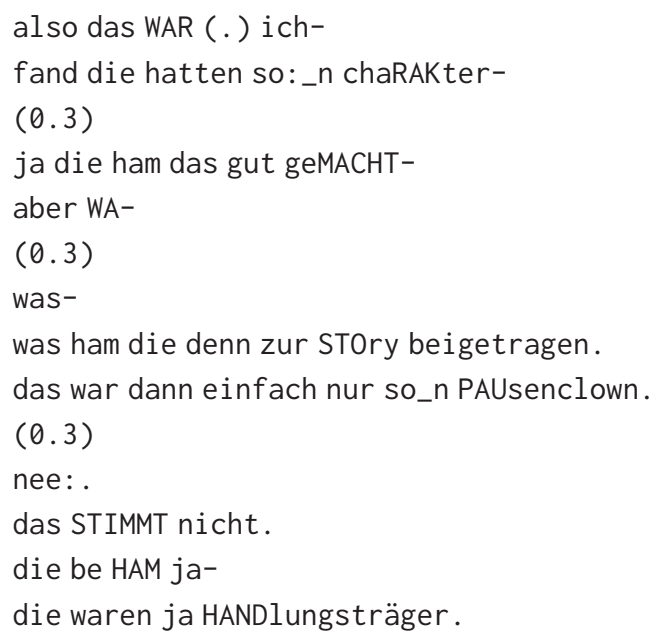

Wird Nichtübereinstimmung von den Beteiligten nicht ausgehandelt, sodass der eine den anderen Gesprächspartner nicht zur Konzession bringen und damit von seiner Meinung überzeugen kann, bezeichne ich diese Form der Nichtübereinstimmung als nicht aufgelöst. Schafft es einer der Gesprächspartner trotz ursprünglich gegenläufiger Bewertungen, im Rahmen einer Bewertungsaushandlung den anderen von seinem Urteil zu überzeugen, wird dies im Folgenden als aufgelöste Nichtübereinstimmung bezeichnet.

Nichtübereintimmung manifestiert sich in den vorliegenden Gesprächen in drei Formen, nämlich als

1. nicht aufgelöste lokale ${ }^{111}$ Nichtübereinstimmung mit Episoden lokaler Übereinstimmung bei globaler ${ }^{112}$ Nichtübereinstimmung,

2. nicht aufgelöste lokale Nichtübereinstimmung bei globaler Übereinstimmung sowie

3. aufgelöste lokale Nichtübereinstimmung bei globaler Übereinstimmung.

\footnotetext{
111 Bei lokaler Übereinstimmung liegen gleichlaufende Bewertungen hinsichtlich eines Teilaspekts oder mehrerer Teilaspekte des Theaterstücks vor. Lokale Nichtübereinstimmung bezieht sich auf gegenläufige Bewertungen der Gesprächspartner im Hinblick auf einen Teilaspekt oder mehrere Teilaspekt des Theaterstücks.

112 Globale Nichtübereinstimmung bezieht sich auf gegenläufige Bewertungen der Gesprächspartner im Hinblick auf das Theaterstück insgesamt.
} 
Diese drei Formen ${ }^{113}$ werden im Nachfolgenden differenzierter dargestellt. Dabei leiten die folgenden Fragen die Analyse: Wie kündigen die Sprecher Nichtübereinstimmung an, und in welchen Formen wird Nichtübereinstimmung sprachlich realisiert? Wie wird Nichtübereinstimmung im weiteren Gesprächsverlauf von den Beteiligten bearbeitet beziehungsweise sequenziell ausgehandelt?

\subsubsection{Nicht aufgelöste lokale Nichtübereinstimmung bei globaler Nichtübereinstimmung}

Das im Folgenden betrachtete Gespräch unterscheidet sich insofern von allen anderen Gesprächen im Datenkorpus, als dass die beiden beteiligten Theaterbesucher die Divergenz ihrer Bewertungen hinsichtlich der Qualität der gesamten Inszenierung sowie auch einzelner Aspekte der Inszenierung bis zum Gesprächsende hin aufrecht erhalten. ${ }^{114}$ Trotz einiger Episoden der Übereinstimmung werden die lokale und globale Nichtübereinstimmung von den beiden Beteiligten nicht aufgelöst und in Übereinstimmung überführt.

Bereits zu Beginn des Gesprächs projiziert Moritz mit „darf ich mich AUFregen?“ und „nomalerweise würd ich jetzt nach HAUse gehn nach d ersten $<<$ lachend>teil>“, was im weiteren Gesprächsverlauf folgt, nämlich die explizite Verbalisierung seines negativen Eindrucks im Hinblick auf das im Stück Dargebotene. Marco hingegen bewertet das Stück zu Beginn des Gesprächs positiv (,bin tatsächlich gespannt worüber du dich AUFregen möchtest. ich fand_s nämlich (.) EIgentlich äh durchaus GUT“). Auch im weiteren Gesprächsverlauf evaluiert Moritz das Theaterstück und seine Einzelaspekte durchweg negativ. Dass die Divergenz der Bewertungen zwischen Marco und Moritz bis zum Ende des Gesprächs aufrechterhalten wird, mag unter anderem mit dem stark divergierenden Wissenshintergrund beziehungsweise Wissenszugang zusammenhängen: Denn während Moritz in Bezug auf Theaterinszenierungen allgemein sowie speziell in Bezug auf die rezipierte Inszenierung von „Hamlet“ über fundierteres Wissen ${ }^{115}$ verfügt, verweist Marco im Gespräch immer wieder explizit auf sein Nicht-Wissen und positioniert sich selbst als relativen Laien sowie Moritz als

113 Als eine vierte Form wäre zudem eine durchweg nicht aufgelöste lokale Übereinstimmung bei globaler Nichtübereinstimmung denkbar, diese Form tritt allerdings in den in dieser Arbeit untersuchten Gesprächen nicht in Erscheinung.

114 Vergleiche zu einem ähnlichen Fall bei einer öffentlichen TV-Diskussion auch Gruber (1993). 115 Moritz ist unter anderem als Regisseur tätig, worauf er im Rahmen des Gesprächs zumindest implizit verweist (siehe Beispiel 74, Z. 115). Auch Marco setzt diese Tatsache im Gespräch an mehreren Stellen relevant (siehe Beispiel 69, Z. 159-161). 
Experten. Im Folgenden soll anhand dieses Gesprächs aufgezeigt werden, wie es den beiden Beteiligten gelingt, die im Hinblick auf die Bewertung des Theaterstücks vorherrschende Nichtübereinstimmung zu bearbeiten ohne die Beziehungsebene zu tangieren oder das Gespräch eskalieren zu lassen.

Die in Beispiel 91 wiedergegebene Situation ereignet sich, nachdem Marco und Moritz ihre Plätze im Theatersaal verlassen und sich auf den Weg ins Foyer des Theaters begeben haben. Marco nimmt in Zeile 067 Bezug auf Moritz' Ankündigung zu Beginn der Aufnahme (vgl. Z. 028), und bewertet anschließend das bisher Rezipierte positiv. Durch eine Subjektivierung, Unschärfemarkierung sowie Disfluenzmarker schwächt Marco seine Globalbewertung ab und markiert diese als potenziell heikel und Face-bedrohend. Anhand der Art und Weise der sprachlichen Realisierung der Bewertung wird zum einen eine Präferenz für Übereinstimmung deutlich, andererseits indiziert Marco, dass er Moritz’ Äußerung zu Beginn des Gesprächs (Z. 028) bereits als negative Bewertung des Theaterstücks deutet. Im folgenden Beispiel wird die im weiteren Gesprächsverlauf vorherrschende Nichtübereinstimmung etabliert:

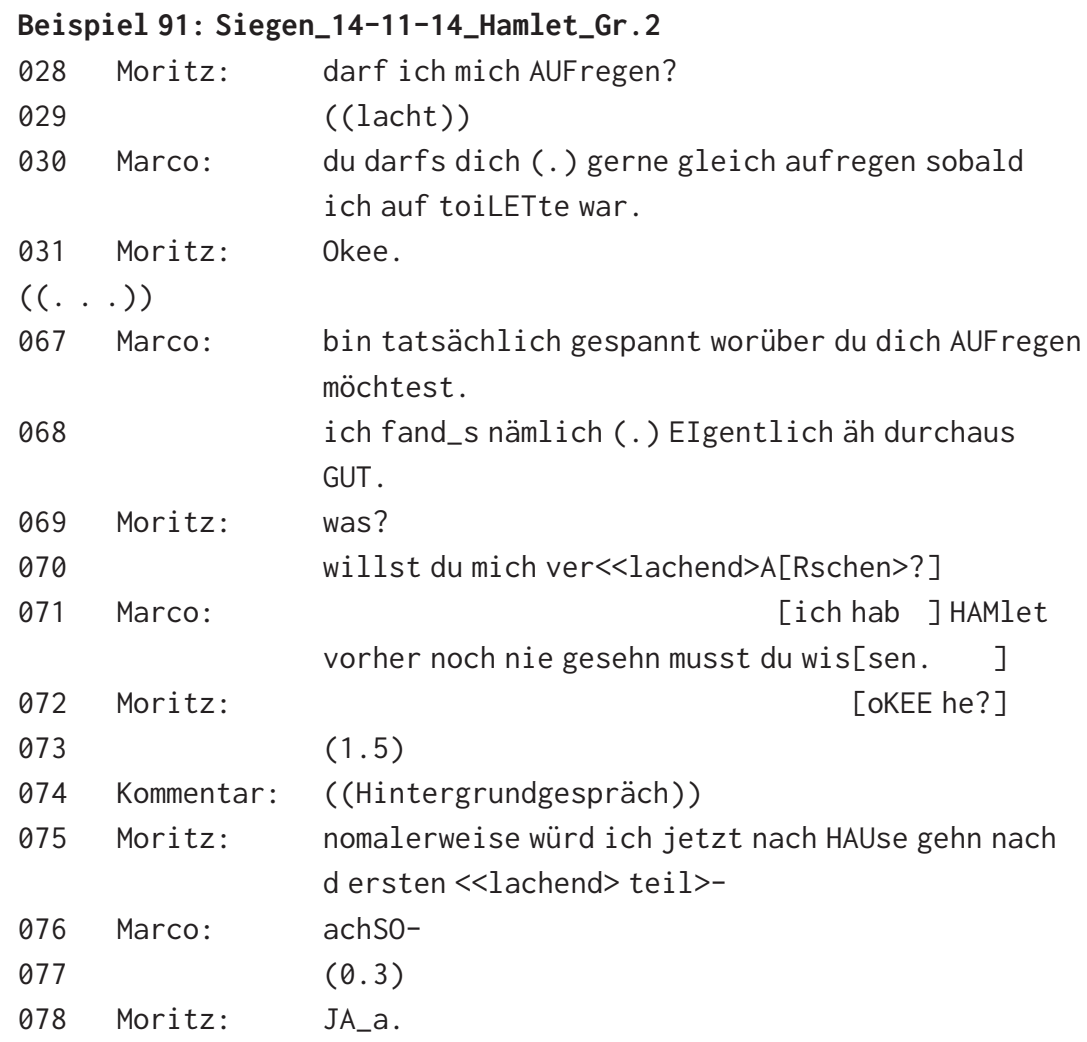




$\begin{array}{ll}079 \text { Marco: } & \text { ja dann erKLÄR gleich mal. } \\ 080 & (2.8) \\ 081 & \text { vor allem waRUM. }\end{array}$

Moritz' Reaktion auf Marcos positive Erstbewertung in Form der von Lachen begleiteten affektiv aufgeladenen und extrem formulierten rhetorischen Frage ,willst du mich ver<<lachend $>$ ARschen $>$ ?“ (Z. 070) projiziert direkt hervorgebrachte Nichtübereinstimmung und löst bei Marco die mit Nachdruck geäußerte Rechtfertigung „ich hab HAMlet vorher noch nie gesehn musst du wissen.“ (Z. 071) aus, mit der er gleichzeitig auf eine vorliegende Wissensasymmetrie verweist. Moritz nimmt dies in Zeile 072 zur Kenntnis und deutet wiederholt eine negative Stellungnahme an (,nomalerweise würd ich jetzt nach HAUse gehn nach d ersten $<<$ lachend> teil〉“, Z. 075). Am Ende des angeführten Ausschnitts fordert Marco Moritz in Zeile 079 zu einer Erläuterung seiner bisher negativen Stellungnahmen im Hinblick auf die Inszenierung auf und fordert mit „vor allem waRUM.“ (Z. 081) explizit eine Begründung ein.

Als sich Marco und Moritz im Theaterfoyer befinden, liefert Moritz die zuvor von Marco eingeforderten Erläuterungen. Im Verlauf des Ausschnitts ${ }^{116}$ zeichnet sich eine Intensivierung der Nichtübereinstimmung ab:

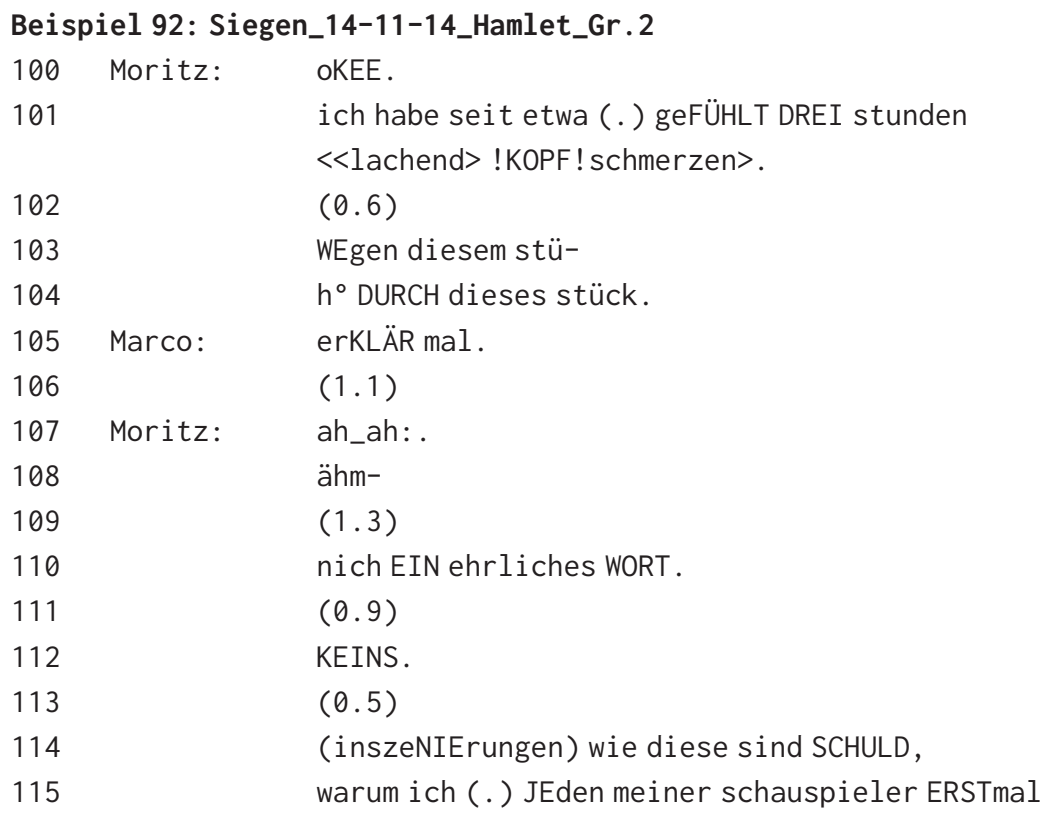

116 Siehe zu einer früheren Analyse dieses Beispiels Hrncal (2018: 279-285). 


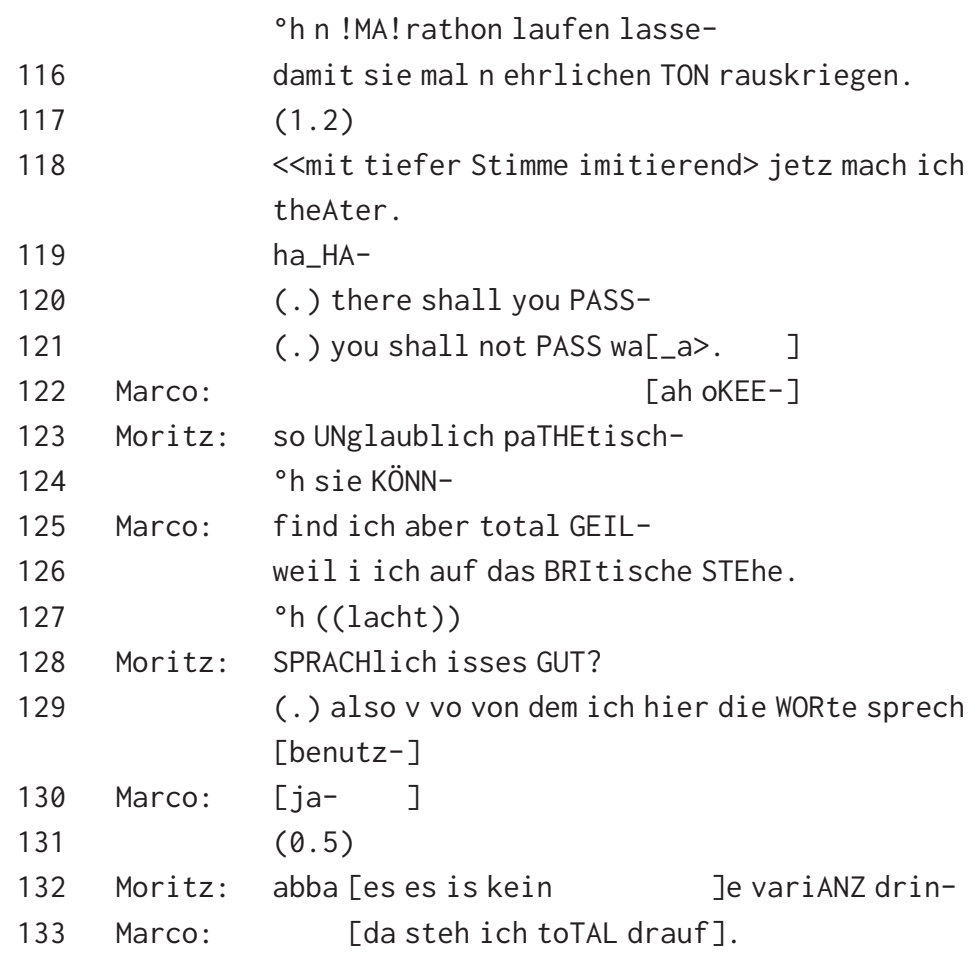

In Zeile 110 moniert Moritz, indem er sich auf die gesamte Inszenierung des Theaterstücks bezieht (,inszeNIErungen wie diese“, Z. 114), dass „nich EIN ehrliches WORT“ (Z. 110) geäußert wurde, und betont dies nachdrücklich mit der akzentuierten Ein-Wort-Äußerung „KEINS“ (Z. 112). Anschließend nimmt Moritz einen Wechsel von der globalen Ebene des Stücks auf die Ebene seiner persönlichen Alltagswelt außerhalb des Stücks vor (,(inszeNIErungen) wie diese sind SCHULD, warum ich (.) JEden meiner schauspieler ERSTmal ${ }^{\circ} \mathrm{h} n$ !MA!rathon laufen lasse- damit sie mal n ehrlichen TON rauskriegen.“, Z. 114-116). An dieser Stelle im Gespräch verweist Moritz erstmals auf seinen Hintergrund als Regisseur einer Theatergruppe („JEden meiner schauspieler“, Z. 115) und den damit verbundenen Wissensstatus als Rechtfertigung seiner Bewertung. Diese begründet er zudem, indem er sich durch die Übernahme der Rollen dargestellter Figuren in die Welt des Stücks projiziert und diese mit seiner Reinszenierung „<<mit tiefer Stimme imitierend $>$ jetz mach ich theAter. ha_HA- (.) there shall you PASS- (.) you shall not PASS wa_a“ (Z. 118-121) parodiert (siehe auch Beispiel 45, Kapitel 7.4). Auf diese Weise indiziert er auch „eine verhältnismäßig große Distanz zum ,Bewertungsobjekt““ (Baldauf 2001: 81). Marco quittiert Moritz’ Parodie mit dem Erkenntnisprozessmarker „ah“ und einem daran 
angeschlossenen „oKEE“ (Z. 122). In Zeile 123 wechselt Moritz wieder auf die Ebene des Theaterstücks, realisiert eine weitere Bewertung („o UNglaublich paTHEtisch“) und beginnt dann mit „'h sie KÖNN-“ (Z. 124) eine weitere Äußerung. Eingeleitet mit einer Subjektivierung in Verbspitzenstellung (vgl. Imo 2012) realisiert Marco ohne Abschwächung eine extrem formulierte Gegenbewertung (,find ich aber total GEIL-“, Z. 125), die er mit persönlichen Geschmacksvorlieben begründet (,weil i ich auf das BRItische STEhe“, Z. 126) und durch die Einschränkung des Gültigkeitsanspruchs weniger angreifbar macht. Die Begründung seiner Bewertung schließt Marco mit einem Lachen ab (Z. 127). Mit seiner positiven Bewertung der sprachlichen Performance der Darsteller (,SPRACHlich isses GUT? (.) also v vo von dem ich hier die WORte sprech benutz“, Z. 128-129) lenkt Moritz erstmals im Gespräch ein. An diese positive Bewertung der sprachlichen Performance schließt Moritz mit seiner Widerspruch indizierenden Äußerung ,abba es es is keine variANZ drin“ (Z. 132) eine weitere negative Bewertung an und nimmt damit einen Wechsel des Bewertungsgegenstands von der sprachlichen Qualität hin zur Varianz vor, während Marco simultan durch die teilweise veränderte, seine Begründung eskalierende Wiederholung „da steh ich toTAL drauf“ (Z. 133) seine vorangehende Bewertung nochmals betont. Zudem fällt auf, dass Marco mit seiner Äußerung direkte Nichtübereinstimmung verbalisiert, ohne diese abzuschwächen. ${ }^{117}$ Realisiert Marco seine globale Positivbewertung zu Beginn des Gesprächs noch in einer abgeschwächten Form (,ich fand_s nämlich (.) EIgentlich äh durchaus GUT") und markiert diese so als potenziell heikel und Face-bedrohend, so greift er bei der Realisierung seiner mit Moritz' Argumenten nicht übereinstimmenden Bewertungen in den Zeilen 125 und 133 nicht mehr auf dieses Verfahren zurück. Diese bereits angesprochene Intensivierung der Nichtübereinstimmung weist darauf hin, dass eine Verschiebung einer anfänglichen Präferenz für Übereinstimmung hin zu einer Präferenz für Nichtübereinstimmung stattgefunden haben könnte (vgl. Kotthoff 1993). ${ }^{118}$

Auch im weiteren Gesprächsverlauf reagiert Moritz nicht auf Marcos wiederholt positive Bewertung der Inszenierung, sondern fährt mit seiner Kritik

117 Vergleiche auch Kotthoff (1993: 204): „It seems that the more positive an initial assessment within a debate context is, the more negative its contextual meaning becomes, due to the downgrading of relevance that is attributed in that special context. This further indicates that preference order has already changed." Kotthoff stützt sich bei ihren Ausführungen auf Streitgespräche aus dem Deutschen sowie dem amerikanischen Englisch.

118 Vergleiche Kotthoff (1993: 203): „When the context of argumentation is established, it is no longer preferred to agree. On the contrary, it seems to be very important to contradict quickly and in a coherent manner“. 
fort, indem er nun - immer noch auf der Ebene des Theaterstücks - die dort präsentierten Rollen zum Bezugsobjekt seiner Bewertungen macht ${ }^{119}$ :

\section{Beispiel 93: Siegen_14-11-14_Hamlet_Gr.2}

134 Moritz: es is (.) so GUT wie keine ROLlenarbeit g geleistet worden-

(. ) das das is SCHUL theaterniveau.

138 Marco ja aber so stell ich mir $<<$ lachend $>$ theAter vor $>$.

140 Moritz: d das das d d das sinkeine ROLlen-= $=$ das $\sin A B$ [ziehbilder. ]

$$
\text { [OKEE- ] }
$$

[ja es GEHT- also ich] find HAMlet schon äh:-

Moritz: [AUßer (also);

Marco: er SELBST ist schon (.) ganz WITZi: ch. (0.7)

Moritz: NEIN nees mh-

(. ) ich MEIne-

Marco: GEHT schon (wieder).

Moritz:

GUCK do mal wie die leute reaGIEren. s_is so <<imitierend> ! OH! mein STICHwort kam. ich KOMM jetz mal auf die BÜHne. !OH! mein STICHwort kam. ich muss jetz die HAND geben. !OH! mein STICHwort kam. ich muss auf den Boden gucken>. 'hh okee sowas fällt mir zum beispiel gar nich AUF . = =aber du bist JA: na DU:als regisSEUR?

163 Moritz: ((Moritz ist 1. Vorstandsvorsitzender und Regisseur eines studentischen Theatervereins))

164 Marco: halt DANN is auchMERKST es wahrscheinlich au einfach mehr-

119 Siehe zu einer früheren Analyse dieses Beispiels Hrncal (2018: 279-285). 
165

166

167

168

169

170

171

172

173

174

175

176 Marco:

177 Moritz:

178 Marco:
$(0.4)$

Moritz: die gesamte inszeNIErung passt $i$ ist nich richtig.

was was was was SOLL das-

$(0.8)$

soll (. ) ALso-

$\mathrm{hh}^{\circ}$

kann i AU nich beurteilen.

hast du HAMlet schonma-

du hasts wahrscheinlich hamlet

[TAUsendma gesehen. ]

[ich hab HAMlet schon_]n etwa f fünf

verschiedenen verSIOnen gesehen-

un[d das is ] mit abstand die SCHLECHteste-

[ja:- ]

${ }^{\circ} \mathrm{hh}[\mathrm{ähm}-$ ]

[ohJEE-]

Im Anschluss an Moritz' negative Bewertung „das is SCHULtheaterniveau“ (Z. 137) widerspricht Marco Moritz' Kritik mit dem Verweis auf persönliche Geschmacksvorlieben (,so stell ich mir <<lachend> theAter vor>“, Z. 138) und verweist so sehr vage und vorsichtig auf Maßstäbe, die seiner positiven Bewertung des Theaterstücks beziehungsweise den von Moritz kritisierten Aspekten zugrunde liegen, macht diese Moritz allerdings nicht zugänglich. Ohne auf Marcos Äußerungen einzugehen fährt Moritz mit der Bewertung der darstellerischen Performance einiger Schauspieler fort (, $d$ das das $d \mathrm{~d}$ das sin keine rollen- $==$ das sin ABziehbilder“, Z. 141). Marco quittiert dies mit einem zur Kenntnis nehmenden „oKEE“ (Z. 142). Nach einer kurzen Pause schließt Marco an Moritz’ zum Teil nicht $\mathrm{zu}$ verstehende Äußerung in Zeile 146 eine weitere subjektivierte Bewertung einer Figur im Stück an, mit der er sich von Moritz' Äußerungen distanziert (,also ich find HAMlet schon ${ }^{120}$ ä:h- er SELBST ist schon (.) ganz WITZi:ch.“), und die er durch die unbetonte Gradpartikel „ganz“ abschwächt. Gleichzeitig

120 Vergleiche den Eintrag zur Funktion von „schon“ als Satzäquivalent (Métrich/Faucher 2009: 763) im Wörterbuch deutscher Partikeln: „drückt eine eher ungern gegebene Zustimmung aus, auf die oft eine Entgegnung folgt. Dem Sprecher geht es zumeist darum, etwas einzuräumen, um eine geplante Erwiderung vorzubereiten [. . . . “ Dieser zitierte Eintrag erfasst allerdings nicht die Funktion von „schon“ in Beispiel 93 als Ressource zur Indizierung von Nichtübereinstimmung als Reaktion auf die Äußerung des Vorgängers und zur Verstärkung der eigenen Position. 
schreibt er Moritz’ affektiv aufgeladener Äußerung „d das das d d das sin keine rollen- = = das sin ABziehbilder“ (Z. 141) den Status einer Negativbewertung zu. Moritz setzt vermutlich zu nicht weiter spezifiziertem Widerspruch an („NEIN nees mh- (.) ich MEIne-“, Z. 148-149). Worauf sich Marcos nachfolgende Äußerung „GEHT schon (wieder)“ (Z. 150) bezieht, ist anhand des Ausschnitts nicht rekonstruierbar. Mit der Matrixsatzkonstruktion „GUCK do mal wie die leute reaGIEren“121 (Z. 151) lenkt Moritz Marcos Aufmerksamkeit vermutlich auf die schauspielerische Leistung der Darsteller und seine folgende Argumentation, die zugleich einer Konkretisierung und Rekonstruktion des Bezugsobjekts als auch der Bewertung dieses Bezugsobjekts („wie die leute reaGIEren“) dient. Dabei übernimmt Moritz die Perspektive der im Stück agierenden Schauspieler (siehe auch Beispiel 46, Kapitel 7.4), parodiert ihre Performance durch seine Imitation „S_is so <<imitierend> !OH! mein STICHwort kam. ich komm jetz mal auf die BÜHne. !OH! mein STICHwort kam. ich muss jetz die HAND geben. !OH! mein STICHwort kam. ich muss auf den BOden gucken ““ (Z. 152-157) und lässt Marco so an seinem individuellen Erleben während der Rezeption teilhaben. Marco steigt in dieses Mokieren allerdings nicht ein, sondern legt erstmals im Gespräch fehlendes Wissen und eine damit verbundene fehlende Kompetenz offen (, ${ }^{\circ} \mathrm{hh}$ okee sowas fällt mir zum beispiel gar nich AUF“, Z. 158). Da Marco der Bewertungsgegenstand hier also gar nicht zugänglich ist, kann er diesen auch nicht (adäquat) bewerten (vgl. Auer 1993: 209). Marco löst das Problem, indem er zuerst sich als (relativen) Laien positioniert und sich so weniger angreifbar macht. Moritz hingegen schreibt er in unmittelbarem Anschluss und im Kontrast zu sich selbst die Rolle des Experten zu (,aber du bist JA:- na DU:- als regisSEUR?“, Z. 159-161). Moritz scheint - ungeachtet Marcos Äußerungen - mit seiner Negativkritik fortzufahren („halt DANN is auch-“, Z. 163). Bevor Moritz seinen nächsten Kritikpunkt äußert, finalisiert Marco mit „MERKST es wahrscheinlich au einfach mehr“ (Z. 164) seine in Zeile 159 begonnene Zuschreibung. Moritz wechselt den Fokus von der zuvor bewerteten Performance der Darsteller zur Inszenierung des gesamten Stücks und damit zurück auf die Globalebene (Z. 166-167). Moritz' aufgeworfene, metareflexive Frage („was was was was SOLL das-“, Z. 167) beantwortet Marco mit dem Verweis auf seine fehlende Beurteilungskompetenz („kann i AU nich beurteilen.“, Z. 171). Mit „hast du HAMlet schonmadu hasts wahrscheinlich hamlet TAUsendma gesehen“ (Z. 172-173) spielt Marco nicht nur auf das ihm fehlende Wissen an, um das Theaterstück wie Moritz beurteilen zu können, sondern auch auf den unterschiedlichen Wissenszugang

121 Vergleiche ausführlicher Günthner (2017: 109) zu Formen und Funktionen univerbierter guck mal- und weißt du-Konstruktionen. 
(vgl. Stivers/Mondada/Steensig 2011: 9), der dem Gespräch zugrunde liegt, und auf dem sich die zwischen Marco und Moritz vorliegende Wissensasymmetrie gründet. Moritz verweist daraufhin auf sein Erfahrungswissen (,ich hab hamlet schon_n etwa f fünf verschiedenen verSIOnen gesehen“, Z. 174) und realisiert eine weitere stark negative Globalbewertung des Theaterstücks (,und das is mit abstand die SCHLECHteste“, Z. 175), die Marco mit der Interjektion „ohJEE“ (Z. 178) quittiert. Während Marco in diesem Ausschnitt die divergierenden Bewertungen mit der zugrunde liegenden Wissensasymmetrie begründet und sich als relativen Laien sowie Moritz als Experten positioniert, nimmt Moritz keine expliziten Selbstzuschreibungen vor. Seine Positionierungen werden vielmehr implizit anhand der von ihm gewählten Formulierungen im „Jargon der Kunstkritik“ (vgl. Hausendorf 2012) (,variANZ“, „ROLlenarbeit“, „SCHULtheaterniveau“ und „ABziehbilder“) deutlich.

Im Anschluss an die Bewertung der Aspekte „schauspielerische Leistung“ und „Bühnenbild“ thematisiert Moritz im folgenden Ausschnitt die Beleuchtung der Bühne. Die Bewertung der Bühnenbeleuchtung als Teilaspekt des Stücks führt Moritz schließlich zu einem Wechsel auf eine metareflexive Ebene:

Beispiel 94: Siegen_14-11-14_Hamlet_Gr.2

216 Moritz: was AUCH stört is das !LICHT! .

217

218

219

220

221

222

223

224

225

226

227

228

229

230

231

232

233
$(0.6)$

sowas was soll das solln das SZEnen sein-=

=also SOLL das-

${ }^{\circ} \mathrm{h}$ weißt du auf einmal sp spielen sie eine realistische SZEneso als ob sie jetz grade an nem ${ }^{\circ} \mathrm{h}$ an nem MEER sindoder an nem an in nem KÖ[nigs]saal sitzen?

Marco: [ja. ]

Moritz: $\quad{ }^{\circ}$ h un dann auf einmal gibt es diesen LICHTswitchund <<imitierend $>$ ! HO ! jetzt bin ich auf der MEtaebene>.

(0.6)

so un DAS aber

Marco: [ja_JA:-]

Moritz: s[o:-a ]lso weder irgendwie beGRÜNdet noch irgendwie-

(0.2)

also es_is ECHT so so ! HM! .=

=jetz machen wir malsch $\mathrm{n}$ bisschen theAter-=

=machen ändern jetzt das LICHT- 
234

235

236

237

238

239

240

241

242

243

244

245

246

247

248

249

250

251

252

253

254

255

256

257

258

259

260

261

262

263

264

265

266

267

268

269

270

271 oh das licht reagiert übrigens auch zu SPÄT$(0.8)$

ALso?

$(0.5)$

es-

(0.7)

ich WEISS nich-

wahrscheinlich ham sie nen andern TECHniker?

(0.3)

Marco: [(war_s) so SCHLECHT- ]

Moritz: [aber das LICHT- ]

(0.2)

[!IST! zu spät- ]

Marco: [! SO! schlecht fand ich_s aber WIR]Klich nich.

Moritz: das LICHT ist zu spät-

UND (. ) sie haben etwa $v$ drei oder vier verschiedne

LICHTstimmungen-

$(0.3)$

sie nutzen (. ) NUR-

(0.6)

ACHT scheinwerfer?

${ }^{\circ} \mathrm{h}$ plus irgendwie nochmal zwei von der BüHne irgendwo-

(1.4)

halt die ham irgendwie so_n oRANGEton da?

$(0.8)$

öh (. ) d dis is die KRÖnung.

der !STUHL! .

$(0.7)$

dieser EIne stuhl. =

=der is beLEUCHtet is in der !PAU! se.

(0.3)

WArum is das ein moderner ! SCHUL! stuhl-

(1.0)

was die haste $n$ RIEsen dieses dieses-

${ }^{\circ} \mathrm{h}$ an!TI!ke alles irgendwie MÖCHtegern-

a ALTmodisch?

$(0.7)$

öh-

un dann has_u_da diesn !SCHUL! stuhl MITtendrin 
stehen.

272

273

274

275
(1.4)

what the !FUCK!?

(0.9)

Mit „was AUCH stört is das !LICHT!.“ (Z. 216) führt Moritz seine listenartig angebrachte Negativkritik fort. Mit seiner In-Frage-Stellung in den Zeilen 218 und 219 leitet er einen Wechsel auf eine metareflexive Ebene ein, setzt zu einem Deutungsversuch an (,also SOLL das“), den er allerdings abbricht und stattdessen Marcos Aufmerksamkeit mit „weißt du“ auf eine weitere Rezeptionserfahrung lenkt (,auf einmal sp spielen sie eine realistische SZEne- so als ob sie jetz grade an nem ${ }^{\circ} \mathrm{h}$ an nem MEER sind- oder an nem an in nem KÖnigssaal sitzen? 'h un dann auf einmal gibt es diesen LICHTswitch-“, Z. 220-222). Anschließend wechselt er in die Rolle des Regisseurs und spricht mit dessen Stimme (,und <<imitierend> !HO! jetzt bin ich auf der MEtaebene>.“, Z. 225, ,jetz machen wir malsch $\mathrm{n}$ bisschen theAter-= = machen ändern jetzt das LICHT-“, Z. 232-233, siehe auch Beispiel 47, Kapitel 7.4). Dabei bringt Moritz „nicht nur die Perspektive der zitierten Figur“, sondern gleichzeitig auch seine eigene Perspektive zum Ausdruck, sodass es zu einer Überlagerung der „Stimme der zitierten Figur“ und der „Evaluation des/der Zitierenden“ kommt (Günthner 2002: 62-63). Im Anschluss daran nimmt Moritz wieder seine Rolle als kritischer Theaterzuschauer ein und moniert die mangelnde Nachvollziehbarkeit (,,also weder irgendwie beGRÜNdet noch irgendwie-“, Z. 229) sowie das seiner Meinung nach unpassende Timing des Lichtwechsels (" $\mathrm{h}$ das licht reagiert übrigens auch zu SPÄT-“, Z. 234). Weitere, durch Disfluenzmarker verzögerte Formulierungsansätze bricht Moritz ab und schließt diese vorerst mit der Formel „ich WEISS nich“ ab, die vermuten lässt, dass ihm weitere Erklärungen in diesem Moment nicht zugänglich sind. Dass auch Marco Moritz' Erläuterungen vermutlich als vorerst abgeschlossen und Moritz' Imitationen damit als Bewertung deutet, wird an seiner Nachfrage „(war_s) so SCHLECHT-“ (Z. 243) deutlich. In Überlappung mit dieser Nachfrage von Marco weist Moritz nochmals darauf hin, dass das Licht zu spät ist (Z. 244-246). Der Moritz' Bewertung widersprechende Einwand Marcos (,!!SO! schlecht fand ich_s aber WIRKlich nich.“, Z. 247) wird von Moritz ignoriert, der stattdessen weitere minutiöse Erläuterungen der Bühnenbeleuchtung liefert (Z. 248-256). In Zeile 258 und 259 greift Moritz mit „öh (.) d dis is die KRÖnung. der !STUHL!.“ ein bereits zu Beginn des Gesprächs thematisiertes Element des Bühnenbilds auf und führt kritisierend an, dass dieser moderne „!SCHUL!stuhl“ (Z. 264) nicht zum „an!TI!ke alles irgendwie MÖCHtegern- a ALTmodisch“ (Z. 267-268) gehaltenen Bühnenbild passt. In diesem Ausschnitt nimmt Moritz, 
angestoßen durch eine Bewertung auf der ästhetisch-performativen Ebene des Stücks, zweimal einen Wechsel auf eine metareflexive Ebene vor, um bestimmte Aspekte der Inszenierung zu deuten beziehungsweise mögliche Gründe für die Art der Inszenierung zu finden. Marco geht darauf allerdings nicht ein, sondern quittiert Moritz' Äußerungen mit Hörerrückmeldungen und dem Hinweis, dass ihm dies ,alles nich <<lachend> AUFgefallen>“ (Z. 275) sei. Somit kommt es aufgrund der - an dieser Stelle von Marco verbalisierten - asymmetrischen Rezeptionserfahrungen zu keiner gemeinsamen Deutungsaushandlung.

Wie auch im Gespräch zwischen Viktoria und Donata (siehe Beispiel 88) initiiert Moritz nach der ausbleibenden Beteiligung seines Gesprächspartners an einer metareflexiven Deutung einen Themen- und Ebenenwechsel, indem er Marco durch das Aufgreifen seiner Deklaration ,is mir alles nich <<lachend> AUFgefallen >“ (Z. 275) als Interrogativsatz (Z. 276) direkt adressiert, seine Aufmerksamkeit auf das Kostüm einer im Stück agierenden Figur auf der ästhetischperformativen Ebene lenkt und ihm somit eine neue Möglichkeit der Beteiligung am Gespräch einräumt:

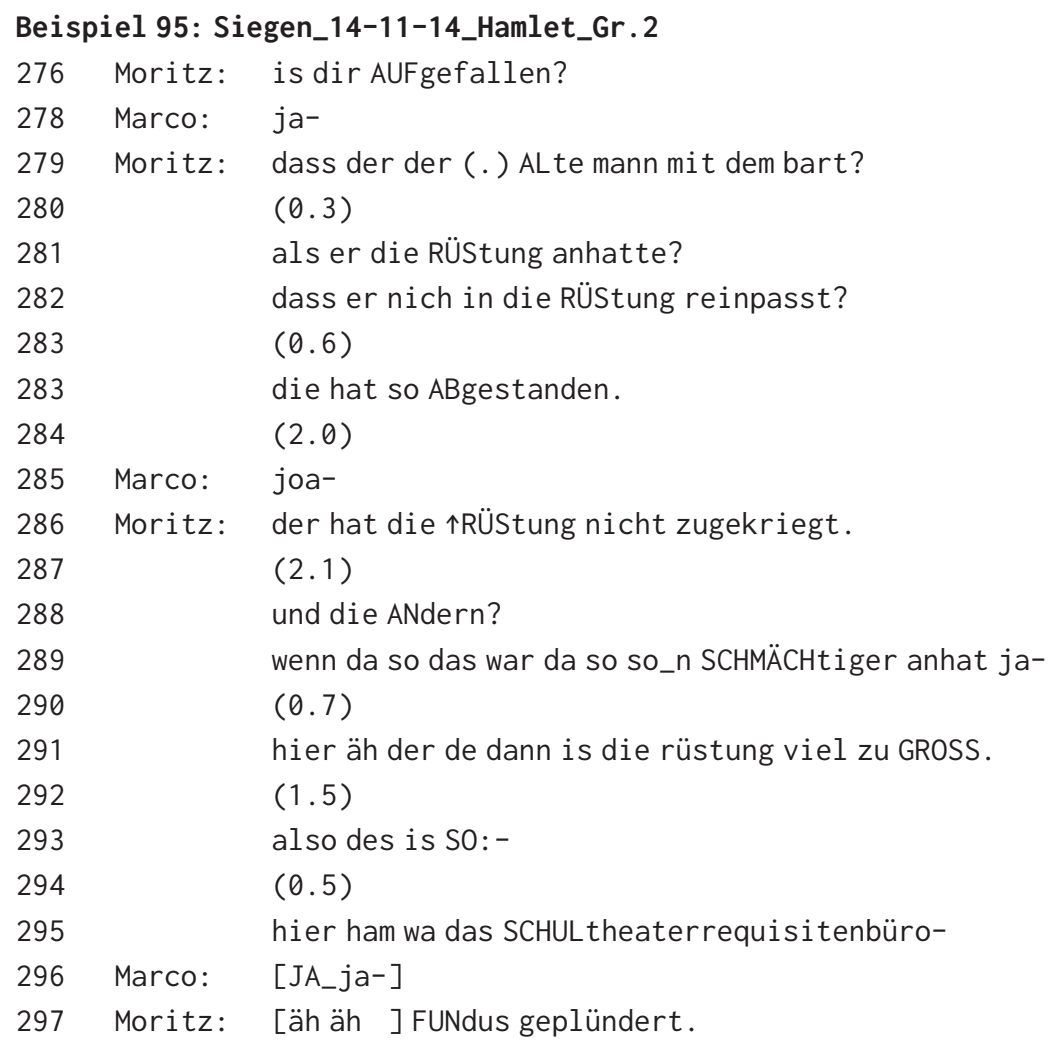


298

299

300

301

302

303

304

305

306

307

308

309

310

311

312

313

314
(1.5)

un dann_s (. ) es is halt überHAUPT nich konsistent.

$(0.5)$

es IS-

auf der einen seite möchte_s irgendwie halt

! ALT ! modisch sein und irgendwie auf TRAditionell machen-

$(0.7)$

dann kommt aber einer [in SO]cken daher-

Marco:

$[\mathrm{ja}-]$

Moritz: also AUCH-

(0.5)

Marco: [oKEE ja:- ]

Moritz: [de gute GEIST- ]

(0.2)

also ! ICH! persönlich seh den geist halt nicht so LEIdend.

KANN man machen.

MUSS man aber nich.

Von Zeile 276 bis 291 schildert Moritz seine Rezeptionserfahrungen mit Bezug auf die Kostüme einiger Schauspieler und kommentiert dies durch die ironische Diskreditierung „hier ham wa das SCHULtheaterrequisitenbüro- äh äh FUNdus geplündert.“ (Z. 295/297). Trotz einiger längerer Gesprächspausen zwischen Moritz’ Äußerungen (Z. 284, Z. 287, Z. 292, Z. 298) knüpft Marco nicht an Moritz' Äußerungen an, sondern reagiert nur minimal (Z. 285, Z. 296, Z. 305). In Zeile 299 rekurriert Moritz nach einer Pause auf den für ihn im Stück vorherrschenden Widerspruch „modern“ versus „traditionell“ beziehungsweise abwertend „altmodisch“, den er bereits in Beispiel 94 mit Bezug auf den in der Pause auf der Bühne stehenden, angeleuchteten Stuhl angeführt hat und versucht nochmals, die Inszenierung zu deuten (Z. 301-306). Marcos daraufhin geäußertes „oKEE ja:“ (Z. 308) indiziert scheinbar Nachvollziehbarkeit, durch die prosodische Realisierung allerdings auch Gleichgültigkeit. In Überlappung mit Marcos Äußerung verweist Moritz auf eine weitere im Stück agierende Figur (Z. 309), distanziert sich von der Art und Weise ihrer Inszenierung, und schränkt den Gültigkeitsanspruch seiner Äußerung sowohl durch den Disclaimer ,also !ICH! persönlich“ als auch durch die angeschlossene Floskel „KANN man machen. MUSS man aber nich“ (Z. 313-314) ein. 
Während sich Marco in den vorangehenden Ausschnitten an einer Aushandlung von Bewertungen, Rekonstruktionen und Deutungen kaum beteilgt, zeigt er sich im nachfolgenden Ausschnitt, in dem es um die Bewertung der von Moritz angesprochenen Figur des im Stück inszenierten Geistes geht, involvierter:

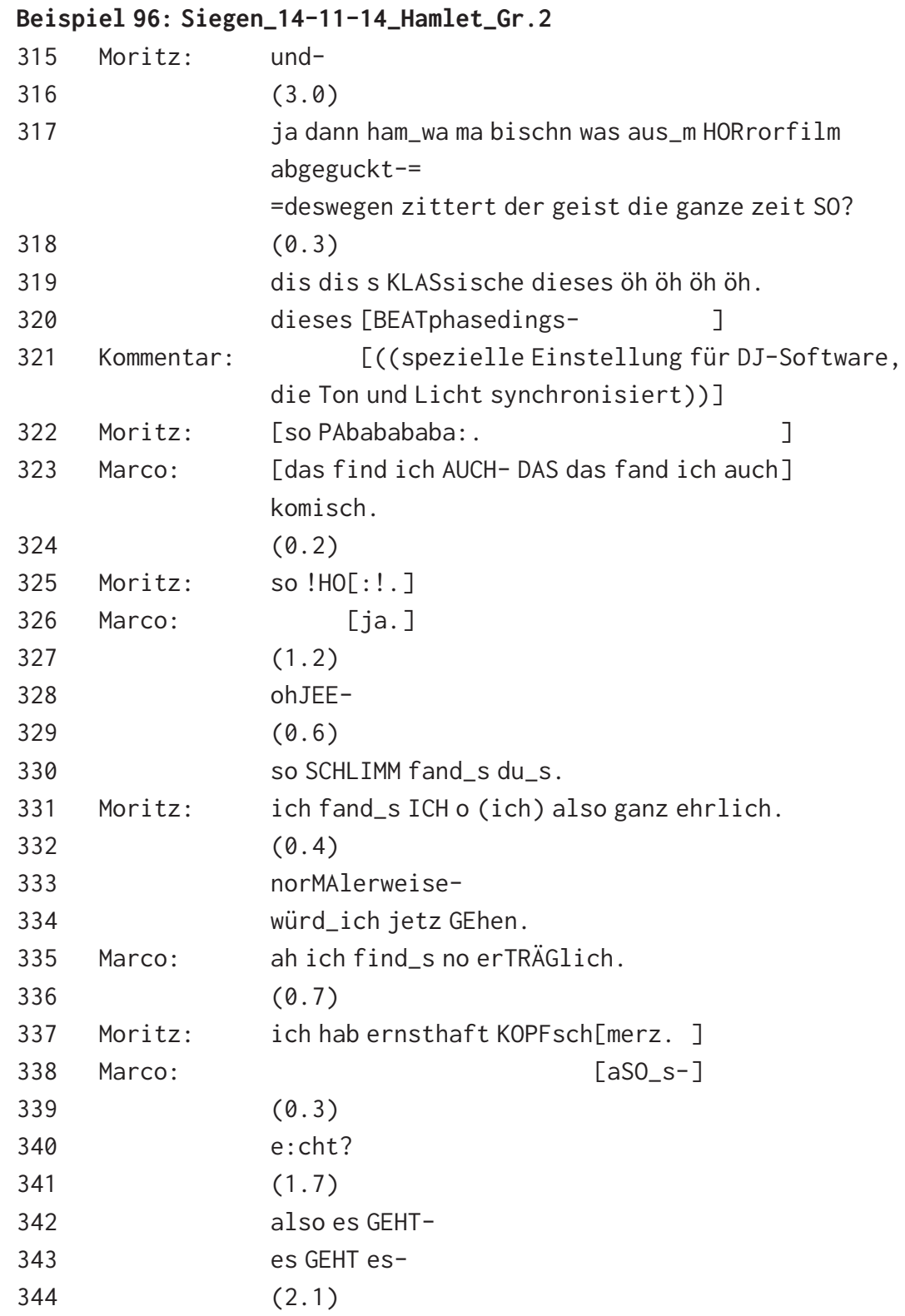


tz (. ) so für PROfessionelles theatervielLEICHT ab[a: - ]

Moritz:

[also f] ür

!PRO!fessionelle[s theAter?]

Marco: [ja: . ]

$(0.2)$

Moritz: ich WEISS_es [nich. ]

Marco: [aber so jetz] ma ABgesehen daalso jetz ma wenn du wenn du mal irgendwie (.) das beiSEIte lässt isses jetz aufes is KEIN wä also.

(0.6)

Moritz: is WAS?

Marco: für dich klingt_s ja wie_n WELTuntergang.

Moritz: ja.

$(0.5)$

wie gSAGT.

ich würde ! GE! hen.

Zu Beginn des Gesprächsausschnitts reinszeniert Moritz weitere Rezeptionserfahrungen, die er mit einem Wechsel in die und einem Sprechen aus der Perspektive der hinter dem Theaterstück stehenden Akteure einleitet (,ja dann ham_wa ma bischn was aus_m HORrorfilm abgeguckt-== deswegen zittert der geist die ganze zeit SO? (0.3) dis dis s KLASsische dieses öh öh öh öh. dieses BEATphasedings- so PAbabababa“, Z. 319-322). Marco realisiert im Anschluss an Moritz' Reinszenierungen eine übereinstimmende Zweitbewertung (,das find ich AUCH- DAS das fand ich auch komisch.“, Z. 323), mit der er Moritz’ Äußerungen den Status einer (Erst-)Bewertung zuschreibt. Dass Marco in seiner Zweitbewertung das Bezugsobjekt („DAS“) und nicht etwa das evaluierende Adjektiv „komisch“ durch den Hauptakzent hervorhebt, spricht möglicherweise dafür, dass Marco diese Episode der Übereinstimmung im Gespräch, das von einer ansonsten durchgängigen Divergenz der beiden Sprecher hinsichtlich ihrer Globalbewertung des Theaterstücks geprägt ist, herausstellt. Moritz' daran anschließend geäußerte, die Inszenierung parodierende Interjektion „so !HO:!“ (Z. 325) quittiert Marco erneut (siehe Beispiel 93) mit der Interjektion „ohJEE“ (Z. 328) und der Bewertungsunterstellung „so SCHLIMM fand_s du_s.“ (Z. 330), die Moritz sogleich mit der Bemerkung ,ich fand_s ICH o (ich) also ganz ehrlich. (0.4) norMAlerweise- würd_ich jetz GEhen.“ (Z. 331-334) bestätigt. 
Marcos Bewertung „ah ich find_s no erTRÄGlich.“(Z. 335) läuft Moritz’ impliziter Bewertung („ich hab ernsthaft KOPFschmerz.“, Z. 337) zwar entgegen, ist aber im Gegensatz zu seiner eingangs des Gesprächs vorgenommenen Globalbewertung hinsichtlich der Qualität des Theaterstücks in abgemilderter Form („no erTRÄGlich“) realisiert. Zudem bleibt vage, ob Marco auf den gerade von Moritz kritisierten Aspekt der technischen Umsetzung („dieses BEATphasedings“) oder auf das gesamte Stück beziehungsweise die gesamte Inszenierung referiert. Mit seinem Verweis „ich WEISS_es nich.“ in Zeile 350 markiert Moritz seine vorherigen Äußerungen als unscharf und indiziert möglicherweise auch, dass ihm (weitere) Begründungen seiner Bewertung(en) nicht zugänglich sind. Ab Zeile 351 versucht Marco stockend mit „aber so jetz ma ABgesehen da- also jetz ma wenn du wenn du mal irgendwie (.) das beiSEIte lässt isses jetz auf- es is KEIN wä also.“ Moritz doch noch von seiner positiven Bewertung des Stücks zu überzeugen und möglicherweise zu einer Konzession zu bewegen. Vermutlich aufgrund von Marcos Formulierungsschwierigkeiten hakt Moritz mit „is WAS?“ (Z. 355) nach, woraufhin Marco feststellt: „für dich klingt_s ja wie_n WELTuntergang.“ (Z. 356). Diese Feststellung Marcos bestätigt Moritz wiederum durch ein zustimmendes ,ja.“ (Z. 358) sowie durch das wiederholte Aufgreifen seiner bereits geäußerten Anmerkung „wie gSAGT. ich würde !GE!hen.“ (Z. 360-361), was seine negative Globalbewertung nochmals bestärkt.

Im folgenden Ausschnitt findet erstmals eine gemeinsame Bewertungsaushandlung im Hinblick auf einen Teilaspekt der Inszenierung statt. Moritz nimmt in Zeile 454 zwei im Stück agierende Figuren in den Fokus und schildert darauffolgend seine Rezeptionserfahrung:

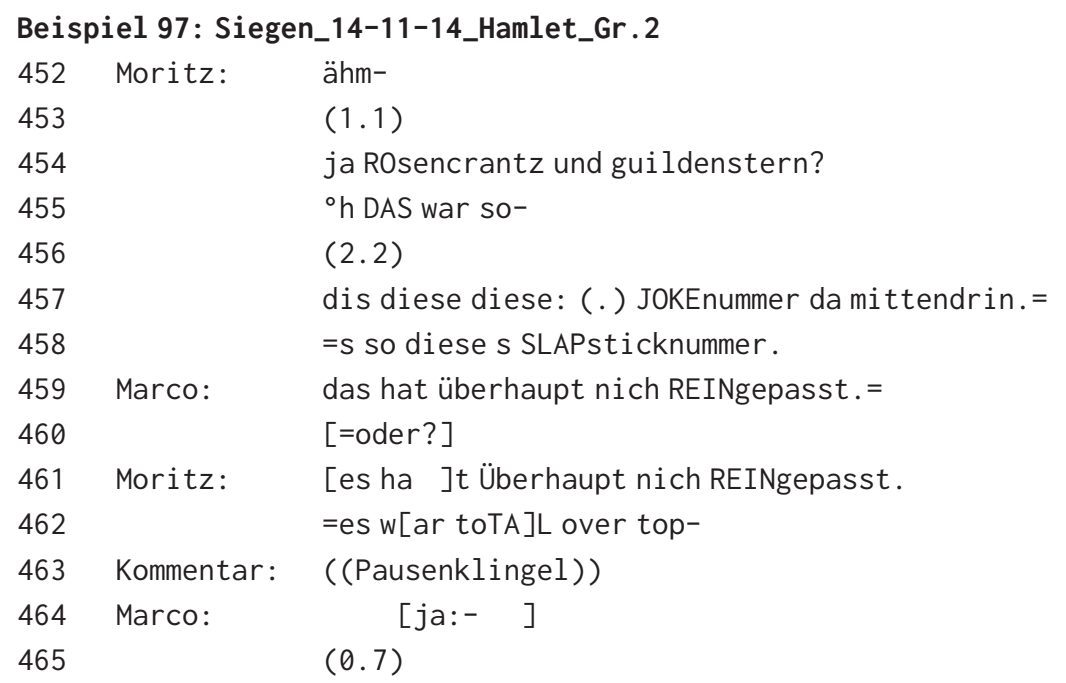


46
Moritz: un es war einfach nur so (. ) oh BITte kommt jetz nich hierher.

aso (.) PEINlich.

so:-

Marco: $\quad j[a ;]$

Moritz: $\quad$ [so] ! END!lich müss mir jetz ma kein TEXT aufsagen-

${ }^{\circ} \mathrm{h}$ so wie_s irgendwie DRINsteht . =also die SPIELen auch irgend

[wie so- geFÜHLT ziemlich den gesamten text? ${ }^{\circ} \mathrm{hh}$ ]

Marco: [so so ZWANGhaft- ja. das hat ich auch den

EINdruckja so zwanghaft AUFgelockert-

${ }^{\circ} \mathrm{h}$ während vorher alles toTAL [äh-]

Moritz:

[ja?]

ENGstirnig äh:-

${ }_{1}$ [geNAU. ]

${ }_{1}[$ wir sin jetz] ma großes theAter gespi

${ }_{2}$ [wör ja-

Moritz: $\quad{ }_{2}$ [und WIE lockern wir_s] auf-

${ }^{\circ} \mathrm{h}<<$ mit verstellter Stimme $>$ indem wir bisschen dumm RUM $>-$

$(0.7)$

<<mit verstellter Stimme> HIhihi.

${ }^{\circ} \mathrm{h}$ ui [UIo>. ]

Marco: [mh. ]

Moritz: $\quad$ öhm um rum äh äh öh LAUtö: generieren.

$(0.5)$

Marco: a: BISSchen lustig war_n sie.

((lacht))

Moritz: ja: .

Marco: ((lacht))

Moritz: $\quad{ }^{\circ} \mathrm{h} \mathrm{ABba-}$

Marco: $\quad{ }^{\circ} \mathrm{h}$

Moritz: ja also DA haste gemerkt so okee. sie KÖNNten_s ja: .

[mh- ]

[also die SCHAU]spieler sind in der LAge dazu auch mal (. ) irgendwie was zu SPIElen.= =irgendw was zu MAchen . = 


\begin{tabular}{|c|c|c|}
\hline 500 & & =IRgendwie ma- \\
\hline 501 & & ${ }^{\circ} \mathrm{h}$ ma das ganze leBENdig zu machen. \\
\hline 502 & & $(0.9)$ \\
\hline 503 & Marco: & [ah_ja GUT aber i- ja: . ] \\
\hline 504 & Moritz: & ] die ganze zeit nur RUM. \\
\hline 505 & Moritz: & ${ }^{\circ} \mathrm{h}$ sie stehen DA und sagen TEXT auf. \\
\hline 506 & & ${ }^{\circ} \mathrm{h}$ okee es is_n BISschen MEHR als text aufsagen. \\
\hline 507 & & $\begin{array}{l}\text { s si (.) also es is (.) nich GANZ schul thema } \\
\text { (the) } \operatorname{ater(.)mäßig.~}\end{array}$ \\
\hline 508 & Marco: & ja_Aba- \\
\hline 509 & & $(1.3)$ \\
\hline 510 & Moritz: & paTHEtisch- \\
\hline 511 & & $(1.4)$ \\
\hline 512 & & KEIN ehrlicher TO:N- \\
\hline 513 & & $(0.4)$ \\
\hline 514 & & KEIne ROLlenarbeit- \\
\hline 515 & & GANZ FURCHTbar. \\
\hline 516 & & $(1.0)$ \\
\hline
\end{tabular}

Moritz’ Rekonstruktion der Performance der beiden Figuren „ROsencrantz und guildenstern“ (Z. 454-458) wird von Marco mit dessen Feststellung „das hat überhaupt nich REINgepasst. =oder?“ (Z. 459) vervollständigt. Der unmittelbare Anschluss dieser Äußerung an Moritz' Rekonstruktion lässt darauf schließen, dass Marco Moritz' eigentlich beschreibenden Ausführungen eine Wertung zuschreibt beziehungsweise sie als Projektion einer Bewertung interpretiert und dieser Bewertung nun zuvorkommt. Mit dem an seine Äußerung angeschlossenen Rückversicherungssignal „oder“ fordert Marco einerseits eine Stellungnahme von Moritz ein, andererseits schreibt er Moritz damit die epistemische Autorität und so auch die Kompetenz zur Bewertung zu. Moritz bestätigt mit dem Wiederaufgreifen von Marcos Worten (,es hat überHAUPT nich reingepasst.“, Z. 461) dessen Bewertung und eskaliert sie mit seiner Zweitbewertung „es war toTAL over top-“ (Z. 462). Dieser Zweitbewertung schließt Moritz eine bereits mit Wertung aufgeladene Erlebensdeklaration (,un es war einfach nur so“) mit einer nachfolgenden pseudokommunikativen Äußerung an („oh BITte kommt jetz nich hierher.“, Z. 466) (vgl. Baldauf 2001: 70 sowie Kapitel 3.1) und verbalisiert seine negative Bewertung nachfolgend explizit mit „aso (.) PEINlich.“ (Z. 467). In direktem Anschluss spricht Moritz auf ironisierende Art und Weise aus der Perspektive der Darsteller beziehungsweise der Figuren (,so !END! lich müss mir jetz ma kein TEXT aufsagen- ${ }^{\circ} \mathrm{h}$ so wie_s irgendwie DRINsteht.“, Z. 470-471), und kommentiert dann aus der Metaperspektive „also die SPIELen 
auch irgendwie so- geFÜHLT ziemlich den gesamten text?“ (Z. 472-473). Die Vervollständigung von Moritz’ Kommentar wird von Marco in Überlappung durch die Schilderung „,so so ZWANGhaft- ja. das hat ich auch den EINdruck- ja so zwanghaft AUFgelockert- ${ }^{\circ} \mathrm{h}$ während vorher alles toTAL äh- ENGstirnig äh:-“ (Z. 474-475) antizipiert, was Moritz durch ein zustimmendes „geNAU“ in Zeile 481 quittiert. Während Marco seine Bewertungen im bisherigen Gesprächsverlauf größtenteils durch ich find_s/fand_s $X$ oder in Verbspitzenstellung mit find ich aber $X$ in Form von nicht näher begründeten, persönlichen Geschmacksurteilen realisiert hat, übernimmt er in Zeile 480 durch das Sprechen aus der Perspektive der hinter dem Theaterstück stehenden Akteure mit „wir sin jetz ma großes theAter gespi wör ja-“ erstmals den reinszenierenden und parodierenden Stil von Moritz. Dieser schließt prompt eine weitere parodierende Reinszenierung an, indem er in die Perspektive der Schauspieler wechselt und mit deren Stimme spricht (,und WIE lockern wir_s auf- ${ }^{\circ} \mathrm{h}<<$ mit verstellter Stimme $>$ indem wir bisschen dumm RUM >- (0.7) <<mit verstellter Stimme > HIhihi. ${ }^{\circ} \mathrm{h}$ uiUIo $>$. öhm um rum äh äh öh LAUtö: generieren.“, Z. 481-487). Der Abschnitt von Zeile 452 bis 487 ist erstmals im Gespräch durch Übereinstimmung hinsichtlich der Bewertung der Inszenierung der im Theaterstück agierenden Figuren gekennzeichnet, wird aber, eingeleitet durch Marcos Äußerung „a: BISSchen lustig war_n sie.“ (Z. 489) und Moritz’ Reaktion auf diese Äußerung wieder in Nichtübereinstimmung überführt. Moritz stimmt Marco zwar mit einem „ja:“ (Z. 491) zu und erläuert dann, dass die Schauspieler „in der lage dazu“ seien, „auch mal (.) irgendwie was zu SPIElen“ (Z. 498), schränkt diese Zustimmung jedoch durch seinen Einwand ,abba (.) sie stehn die ganze zeit nur RUM. ${ }^{\circ} \mathrm{h}$ sie stehen DA und sagen text auf.“ wieder ein. Mit der abgeschwächten, seine vorherige Bewertung (siehe Beispiel 93) relativierenden Äußerung „okee es is_n BIschen mehr als text aufsagen. s si (.) also es is (.) nich GANZ schulthema (the)ater(.) mäßig.“ (Z. 506-507) indiziert er zwar eine Konzession, bewertet dann aber abschließend entweder die Inszenierung in Gänze oder die im unmittelbar vorangehenden Gesprächsverlauf thematisierte Inszenierung der Figuren beziehungsweise ihre darstellerische Leistung mit „paTHEtisch- (1.4) KEIN ehrlicher to:n- (0.4) KEIne rollenarbeit- ganz FURCHTbar.“ (Z. 510-515).

Eingangs dieses Kapitels wurde die Frage aufgeworfen, wie es den Beteiligten gelingt, die durchweg im Hinblick auf die Bewertung des Theaterstücks vorherrschende Nichtübereinstimmung beizubehalten, ohne die Beziehungsebene zu tangieren oder das Gespräch eskalieren zu lassen: Erstens ist festzuhalten, dass bereits zu Beginn des Gesprächs von Marco eine Rollenzuweisung vorgenommen wird, indem er sich selbst als relativen Laien und Moritz als Experten positioniert. Mit dieser Rollenzuweisung indiziert Marco auch, dass die beiden Sprecher nicht über den gleichen Wissenszugang verfügen und somit eine 
Wissensasymmetrie und damit auch eine divergierende Bewertungsgrundlage vorliegen. Denn während Moritz als Regisseur einer Theatergruppe tätig ist und zudem bereits einige Inszenierungen des von Marco und Moritz besuchten Theaterstücks Hamlet rezipiert hat, verfügt Marco weder über das Expertenwissen eines Theaterregisseurs, noch über das Erfahrungswissen der vorherigen Rezeption einer Hamlet-Inszenierung. Gerade dieser fehlende Wissenshintergrund als Basis für eine argumentative Untermauerung und Aushandlung der Bewertungen führt vermutlich dazu, dass Marco sich zumindest zu Beginn des Gesprächs kaum an der Aushandlung der von Moritz initiierten Bewertungen und Deutungen beteiligt. Ein von Moritz initiierter Wechsel vom Deuten auf einer metareflexiven Ebene hin zu einer Fokussierung eines Bezugsobjekts auf der ästhetisch-performativen Ebene, das Marco durch seine Rezeptionserfahrung zugänglich ist, räumt letzterem eine größere Beteiligungsmöglichkeit am Gespräch ein. Beispiel 96 und 97 zeigen, dass es zu einer gemeinsamen Realisierung und Aushandlung von Bewertungen kommt, wenn ein Bezugsobjekt auf der ästhetisch-performativen Ebene des Theaterstücks in den Fokus rückt und beide Gesprächspartner in der Bewertung dieses Bezugsobjekts zumindest vorerst übereinstimmen. An Moritz' Versuch, Marco (wieder) am Gespräch zu beteiligen, wird auch in diesem Gespräch der kooperative Charakter der von den Beteiligten im Pausengespräch betriebenen Kunstkommunikation deutlich. Des Weiteren spricht Moritz' Vorgehen dafür, dass es ihm nicht darum geht, sich als Kenner sozial von Marco zu distinguieren, sondern dass er an einem gemeinsamen Abgleich der Rezeptionserfahrungen interessiert ist, den er dennoch dazu nutzt, sich als Experten zu positionieren.

Zweitens werden der gesellige Kontext und das Sich-Vergnügen im Gespräch sowohl durch Lachen als auch durch Moritz' teilweise theatrale Reinszenierungen im Stück agierender Figuren relevant gesetzt. Dass Moritz das Pausengespräch auch als Bühne nutzt, um sich darzustellen, weist darauf hin, dass der gemeinsame Theaterbesuch ein geselliges Ereignis darstellt, bei dem es nicht zwangsläufig darum geht, ernsthafte Kunstkritik zu üben und über Meinungen hinsichtlich der Qualität des rezipierten Stücks zu streiten, sondern auch darum, mit dem Kommunikationspartner das eigene emotionale Erleben zu teilen (vgl. Kindt 2007, siehe auch Kapitel 3.1). Moritz' parodierende Inszenierung im Stück agierender Figuren lassen einerseits „die Präsentation lebendiger“ wirken, andererseits trägt dieses „Verfahren zur Kontextualisierung der affektiven Einstellung“ von Moritz „zur fremden Rede beziehungsweise zur Perspektivierung gewisser Personen und deren Handlungen“ bei (Günthner 2002: 66) und dient gleichermaßen „zur Evaluation (in-)adäquaten Verhaltens“, in Moritz' Fall einer Evaluation einer aus seiner Sicht inadäquaten Inszenierung der Figuren. „Da diese indexikalischen Mittel keine inhärente, kontextlosgelöste 
Bedeutung haben“, so Günthner (2002: 66, unter Verweis auf Günthner 2000), „und folglich weniger einklagbar sind als referentielle Zeichen, bilden sie geeignete Mittel zur impliziten Kontextualisierung von Bewertungen“. Des Weiteren trägt der wiederholte Einsatz von Expressiva auch zur psychischen Entlastung der Sprecher bei (vgl. Klemm 2001: 86; siehe auch Kapitel 3.1), die während der Rezeption zum Schweigen angehalten und an ihren Sitz „gefesselt“ sind. Dass die Rezeption eines Theaterstücks also auch zur Qual werden kann, macht Moritz in seinen Äußerungen allzu deutlich. In diesem Zusammenhang gibt das Gespräch auch Aufschluss darüber, wie sich Marco im Gesprächsverlauf Praktiken des Bewertens aneignet: Während er zu Gesprächsbeginn Bewertungen in Form von Geschmacksurteilen realisiert und diese, wenn überhaupt, durch persönliche Vorlieben begründet, übernimmt er in Zügen bei (partiell) übereinstimmenden Bewertungen in Ausschnitt 96 und 97 Moritz' Bewertungsstil, indem er Bewertungen durch Perspektivenwechsel und das Sprechen aus verschiedenen Perspektiven oder durch Beschreibungen des Rezipierten zu begründen versucht. Diese Veränderung in der Realisierung der Bewertungen könnte zudem darauf hinweisen, dass die Bewertungsinteraktion in diesem Gespräch auch dazu dient, Marco Bewertungspraktiken in der Kommunikation im Anschluss an die Rezeption einer künstlerischen Performance zu vermitteln. Die Pausengespräche schaffen somit Ermöglichungsbedingungen, Gesprächspartner an die Praxis der Kunstkommunikation heranzuführen (vgl. auch Habscheid 2018), voneinander zu lernen und Wissensasymmetrien zu beheben.

Drittens lässt sich anhand dieses Gesprächs die mit dem Bewerten verbundene Präferenz für Übereinstimmung oder Nichtübereinstimmung und deren dynamische Anpassung im Gesprächsverlauf beobachten. Während Marco zu Beginn des Gesprächs eine noch vorsichtige und zögerliche, durch Unschärfemarkierungen abgemilderte Globalbewertung der Qualität des Theaterstücks vornimmt und seine Positionierung damit als heikel und Face-bedrohend markiert, äußert Moritz direkt und ohne jegliche „Vorsichtsmaßnahmen“ Nichtübereinstimmung hinsichtlich Marcos Bewertung(en). Dass auch Marco im weiteren Gesprächsverlauf weniger bis gar nicht mehr auf Abschwächungen seiner Bewertungen zurückgreift, könnte ein Indiz für den Wechsel der Präferenz zugunsten von Nichtübereinstimmung sein. Obwohl die im Gespräch vorherrschende Nichtübereinstimmung hinsichtlich der Bewertung des Theaterstücks im vorgestellten Ausschnitt nicht aufgelöst wird, stimmen die beiden Gesprächspartner immerhin darin überein, dass „ihre unterschiedlichen Positionen, zumindest gegenwärtig, nicht aufgelöste [sic!] werden können“ (Gruber 1993: 9). Diese unaufgelöste Nichtübereinstimmung wird jedoch von Moritz und Marco „thematisiert und diskutiert, ohne die Personen, die diese kontroversen Meinungen haben, in den Mittelpunkt des Disputs zu stellen“ (Keppler 1994: 126). Das 
bedeutet, dass im Rahmen der dissenten Sequenzen im Gespräch zwischen Marco und Moritz Nichtübereinstimmung im Hinblick auf den Bewertungsgegenstand kommuniziert wird, ohne sich gegenseitig persönlich verbal anzugreifen beziehungsweise auf die Person bezogene Bewertungen vorzunehmen.

\subsubsection{Nicht aufgelöste lokale Nichtübereinstimmung bei globaler Übereinstimmung}

In Beispiel 98 unterhalten sich Wanja und Nelli in der Pause des Theaterstücks Der Kaufmann von Venedig. Das Gespräch umfasst zwar auch Bewertungssequenzen, die Nichtübereinstimmung bezüglich einzelner Aspekte des Stücks aufweisen, im Gegensatz zum Gespräch zwischen Moritz und Marco stimmen Wanja und Nelli jedoch in der (negativen) Globalbewertung der Inszenierung überein. Die beiden sind gerade auf dem Weg zur Getränketheke im Theaterfoyer, als Nelli ab Zeile 111 noch einmal Bezug auf ihre Bewertungsunterstellung „du FINdest es ja !GANZ! schlimm. =ne?“ nimmt, mit der sie das Gespräch unmittelbar nach Beginn der Aufnahme einleitet (siehe auch Beispiel 71, Kapitel 8.1).

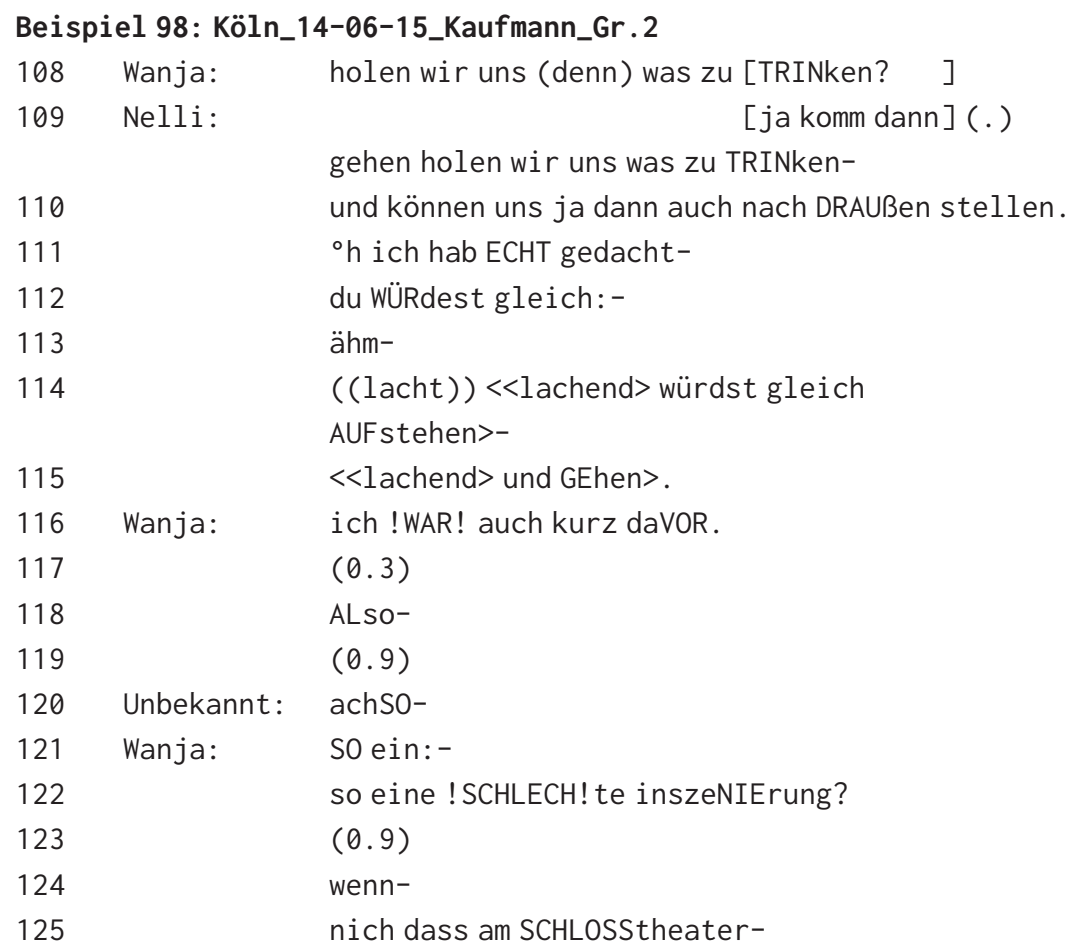


129 Nelli:

130 Kellnerin:

131 Wanja:

132 Nelli:

133

134 Kellnerin:

135

136

137 Wanja:

138 Kellnerin:

152 Kellnerin:

153 Nelli:

154

155

156

157 Kellnerin:

$\begin{array}{lll} & & \text { [euro ZWANzig bit]te. } \\ 158 & \text { Nelli: } & \text { [muss ich SAgen. ] } \\ 159 & \text { Wanja: } & (\text {. }) \text { ja- } \\ 160 & \text { Nelli: } & \text { [DAS äh- ] } \\ 161 & \text { Wanja: } & \text { [moMENT-] } \\ 162 & & \text { hast du[_n BISSchen-] } \\ 163 & \text { Nelli: } & \text { [(ich)- }\end{array}$

alles SUper is?

Aber-

also DAS [is ja ] [echt-]

[HALlo.]

[HAL ]lo-

GRAUenhaft .

ich HÄTte gerne ein ä: hcappucCIno bitte.

JA_a-

(0.4)

den [HERR- ]

[ich HÄTT- ]

ein cappucCIno.

(0.2)

ich hätte gern n SCHNAPS.

(.) ((Lachansatz))

(0.9)

WEIter?

ja (. ) bitte ZWEI cappu-

$(0.4)$

z[wei:.]

[hm: .]

(0.2)

ah-

((Hintergrundgespräche))

(0.7)

noch ein cappucCI[no bit $]\left[\mathrm{te}^{-}\right]$

$\left[{ }^{\circ} \mathrm{h} \quad\right][$ ich FAND $]$ den e-

ich fand den ANfang-=

=den fand ich echt GANZ: ä: h-

fand ich ganz VIELverprech[end- ]

[!FÜNF! ]

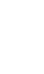




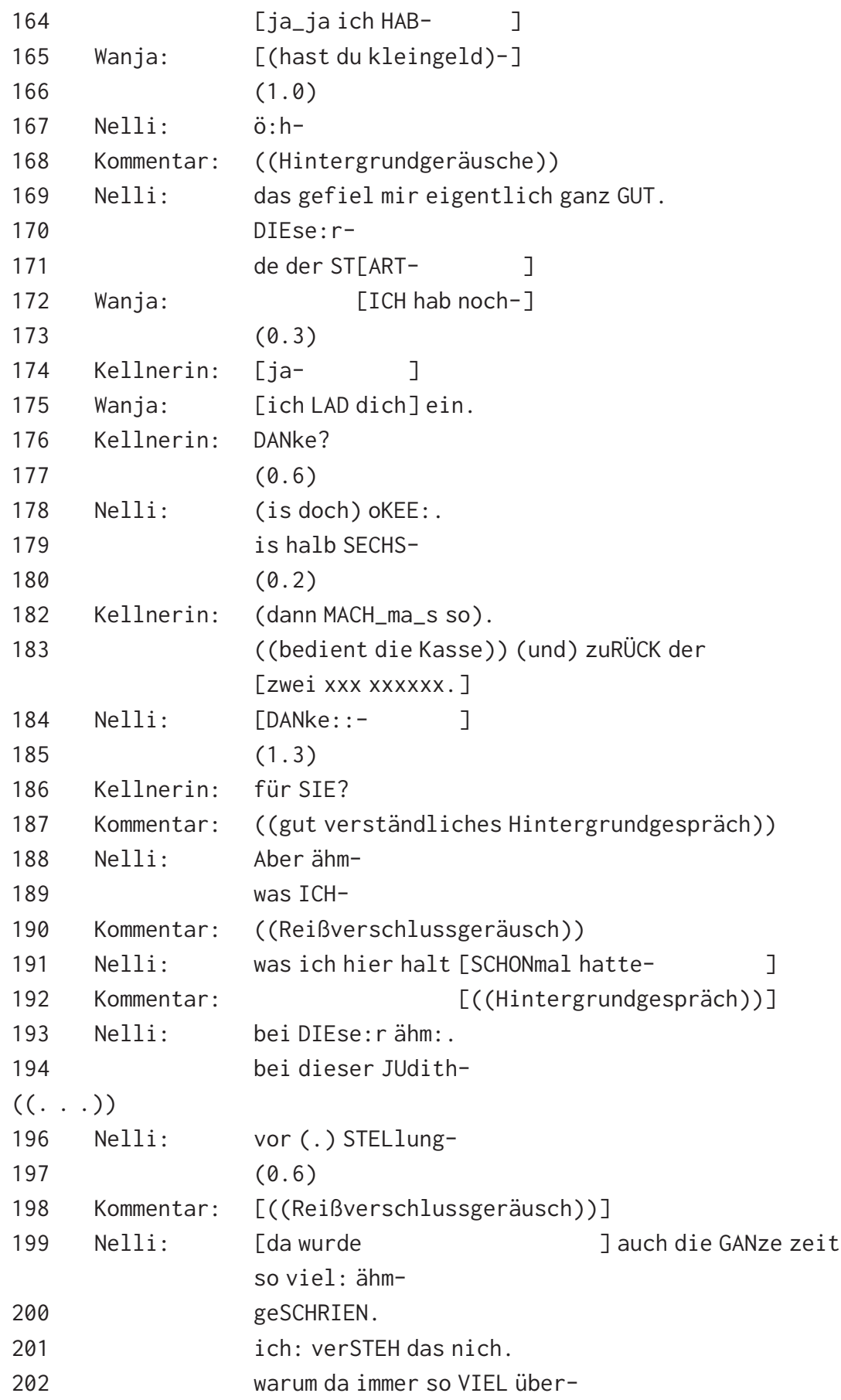


Kommentar: ((laute Hintergrundgeräusche))

Nelli: ähm-

LAUTstärke laufen muss-

das-

[ja. ]

[is mir] toTALauf die NERven gefallen.

(1.8)

also ich DENK-

ich (. ) ich denke wie geSAGT-

ALso-

(0.3)

das stück auf !DREI! stunden AUSzudehnenauch wenn da zwanzig minu[ten PAU]se is-

Nelli:

[ja. ]

das trägt_s halt !ü!berHAUPT nichdiese SINgerei dazwischen?

$(0.6)$

!KOM! plett UNmotiviertja:?

$(0.5)$

und JETZT komma nomma dran (ü)ben(die/hier) MÜSST ich ne: ä:hne LEIter falschrum aufstellen.

ja:?

Nelli:

JA da [(. ) geht- $]$

Wanja:

[MUSS au ]ch dieses-

((stellt Kaffeetasse ab))

dieses geLÄCHter da zwischendurch-

ich fand das kein STÜCK-

in: irgend(ei)ner form iROnisch-

oder LUStig-

oder SONST wie.

(ja_A)?

Kommentar: ((laute Hintergrundgeräusche))

Nelli: $\quad$ ich FIND das g-

ALso.

ich finde man kann das schon durchaus MAchensich dem STÜCK malmoDERner nähern. 
243

244

245

246

247

248

249

250

251

252

253

254

255

256

257

258

259

260

261

262

263

264

265

266

267

268

269

270

271

272

273

274

275

276

278

279 Nelli:

280 Wanja:

281 Nelli:

Nelli: und DAS ähm.

also ich MEIN-

bei shakespeare is ja! VIE! les total WITzig.

äh de (.) das IS ja:-

das find ich das PASS[T ja. ]

Wanja:

[JA ] aber-

[das PASST ja-

[xxx aber ! NICH! wenn] das so mit dem HOLZhammer daherkommt.

ja:?

(1.9)

Kommentar: ((Hintergrundgeräusche))

Nelli: also MIR war das dann-

mir war_s halt dann IRgendwann auch übertrieben-

mit den BEIden.

wobei ich die echt (.) TOLL: fand.

wie synchron die ABgestimmt sind-

DIE ähm: .

Kellnerin: zwei cappucCI[no?]

[h ]ier DIE:-

(0.8)

EIner war !HIER! noch.

bitteSCHÖN?

((stellt Tasse ab))

Kommentar: ((laute Hintergrundgeräusche))

Nelli: gehen ma nach DRAUßen?

Wanja: ja geNAU.

DRAUßen-

(1.9)

oh entSCHULdigung.

(1.0)

ich FINde die beiden:-

die d d die (.) ZWILlinge-

oder die die ZWILlinge.

(.) ö:h-

(0.9)

also WIE die [das-]

[ja: ]

[aber (.) das waren doch MEHR oder weniger-]

[mh wie die das MAchen:? 


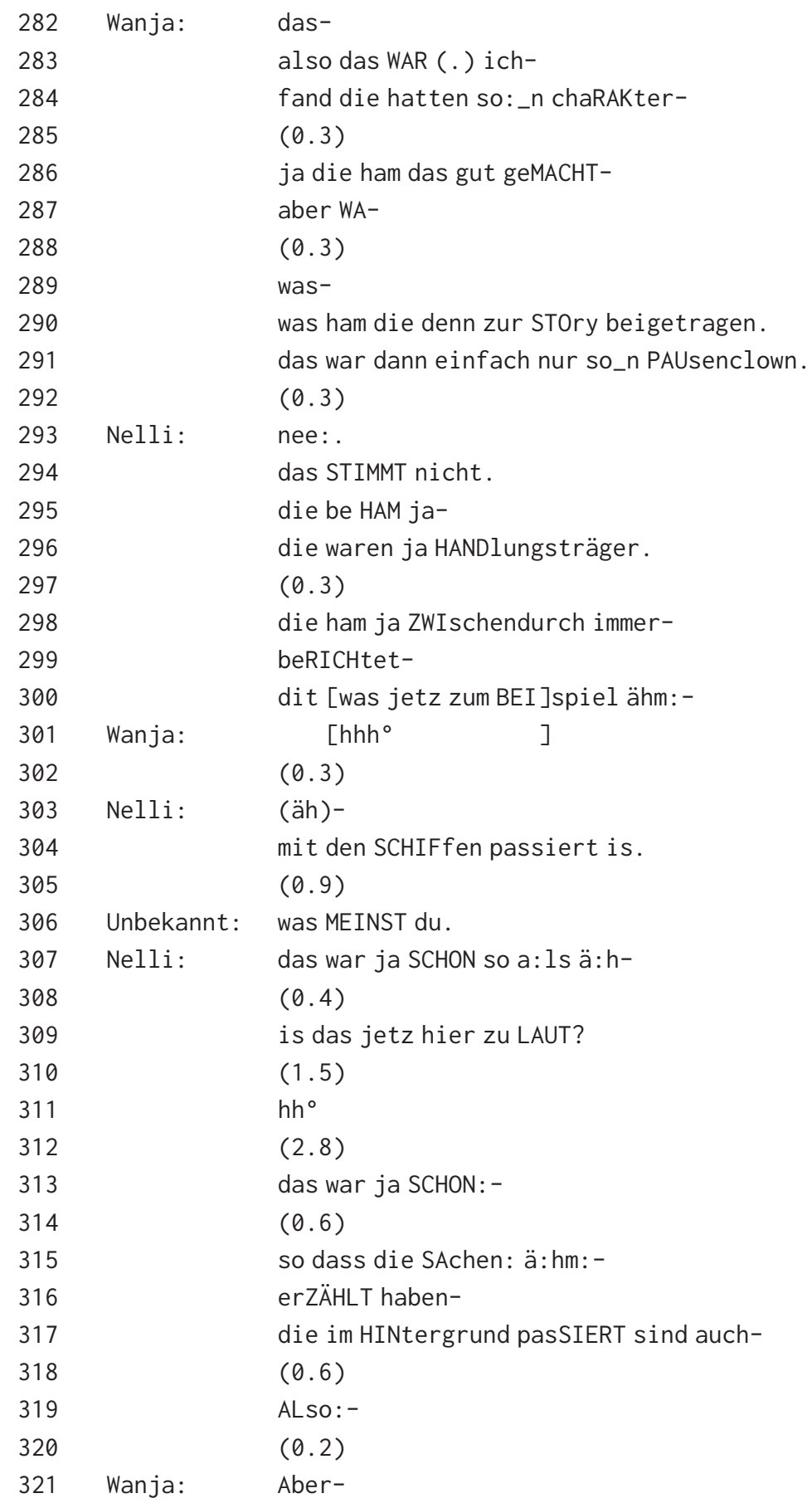


Nelli schildert ab Zeile 111 ihren Eindruck, Wanja wolle den Theatersaal während der laufenden Inszenierung verlassen (,oh ich hab ECHT gedacht- du WÜRdest gleich:- ähm- ((lacht)) <<lachend>du würd(e)st gleich AUFstehen>$<<$ lachend $>$ und GEhen $>$."), und setzt damit eine Reaktion Wanjas relevant, die er in Form einer Bestätigung liefert (,ich !WAR! auch kurz daVOR.“, Z. 116). Im Anschluss an diese Bestätigung von Nellis Wahrnehmung begründet er mit seiner Bewertung „ALso- (0.9) SO ein:- so eine !SCHLECH!te inszeNIErung?“ (Z. 118-122) den Impuls, die Theateraufführung vorzeitig verlassen zu wollen und unterstreicht die erste Bewertung nochmals durch die darauf folgende, ebenfalls sehr negative und extrem formulierte Globalbewertung „also DAS is ja echt GRAUenhaft.“ (Z. 128/131) sowie durch die nicht ernst gemeinte Getränkebestellung „ich hätte gern n SCHNAPS.“ (Z. 140), die vermutlich aber nur an Nelli gerichtet ist. Nach einer kurzen Unterbrechung durch die Getränkebestellung folgt in den Zeilen 153 bis 156 Nellis (Zweit-)Bewertung „”h ich FAND den e- ich fand den ANfang-==den fand ich echt GANZ: ä:h- fand ich ganz VIELverprechend-“. Im Gegensatz zu Wanja, der das bisher Rezipierte einer negativen Globalbewertung unterzieht, äußert Nelli im Hinblick auf den Anfang des Theaterstücks ein milderes Urteil (Z. 153-156). Die Bewertungssequenz wird abermals durch die parallel bearbeitete Getränkebestellung unterbrochen, bevor Nelli mit „das gefiel mir eigentlich ganz GUT. DIEse:r- de der START-“ (Z. 169-171) ihre Bewertung wieder aufgreift. Die explizit positive Bewertung des Beginns des Theaterstücks schwächt Nelli durch den dem Adjektiv „GUT“ vorangestellten Heckenausdruck „eigentlich“ sowie durch die unbetonte Gradpartikel „ganz“ ab und markiert ihre mit der Bewertung vollzogene Positionierung als heikel. Die abgeschwächte sprachliche Realisierung ihrer Bewertung ist auch ein Indiz dafür, dass eine Präferenz für Übereinstimmung vorliegt. Nach einer erneuten side sequence (vgl. Jefferson 1972), in der die Bezahlung der Getränke thematisiert wird, führt Nelli in Zeile 188 bis 209 die Bewertungssequenz fort, indem sie mit dem Verweis auf das Schreien und die Lautstärke einen Aspekt auf der ästhetisch-performativen Ebene des Theaterstücks aufgreift und diesen, wie bereits durch ihr einleitendes „Aber“ (Z. 188) projiziert, negativ bewertet („Aber ähm- was ICH- was ich hier halt SCHONmal hatte- bei DIEse:r ähm:. bei dieser JUdith- vor (.) STELlung- (0.6) da wurde auch die GANze zeit so viel: ähmgeSCHRIEN. ich: verSTEH das nich. warum da immer so VIEL über- ähm- LAUTstärke laufen muss- das- is mir toTAL- auf die NERven gefallen.“). Wanja äußert auf Nellis negative Bewertung der Lautstärke und des Schreiens im Stück vorerst 
keine weitere Bewertung desselben Aspekts, sondern nimmt nachfolgend verschiedene Aspekte auf der Ebene des Stücks beziehungsweise der Inszenierung in den Blick. Er beginnt in Zeile 211 mit der Länge des Stücks (,,also ich DENKich (.) ich denke wie geSAGT- ALso- (0.3) das stück auf !DREI! stunden AUSzudehnen- auch wenn da zwanzig minuten PAUse is- das trägt_s halt !ü!berHAUPT nich-“) und kommt im Anschluss mit „diese SINgerei dazwischen?“ (Z. 219) auf die Gesänge im Stück zu sprechen, die er als „!KOM!plett UNmotiviert-“ (Z. 221) bezeichnet. In direktem Anschluss nimmt Wanja dann eine im Stück vorkommende Leiter in den Fokus, nimmt einen Wechsel in die Rolle der mit der Leiter befassten Figur(en) im Stück vor, spricht mit „und JETZT komma nomma dran (ï)ben- (die/hier) MÜSST ich ne: ä:h- ne LEIter falschrum aufstellen.“ (Z. 224-226) aus deren Perspektive (siehe auch Beispiel 49, Kapitel 7.4) und zieht deren Inszenierung ins Lächerliche. Mit dem „geLÄCHter da zwischendurch-“ thematisiert Wanja einen weiteren Aspekt der Inszenierung und bewertet diesen negativ durch die paraverbal aufgeladene Äußerung ,ich fand das kein STÜCK- in: irgend(ei)ner form iROnisch- oder LUStig- oder SONST wie.“ (Z. 229-235). Nelli distanziert sich in Zeile 240 bis 244 von Wanjas Bewertung (,man kann das schon durchaus MAchen-“) und begründet ihre Position mithilfe der Belehrung „also ich MEIN- bei shakespeare is ja !VIE!les total WITzig. äh de (.) das IS ja:- das find ich das PASST ja.“ (Z. 245-247). Zudem unterstellt sie durch die in ihrer Belehrung enthaltene Partikel ,ja“ gemeinsames Hintergrundwissen. Wanja reagiert auf Nellis Widerspruch mit der in Überlappung sowie als lokale Nichtübereinstimmung realisierten Äußerung „JA aber- xxx aber !NICH! wenn das so mit dem HOLZhammer daherkommt. ja:?“ (Z. 248-250). Der in Wanjas Äußerung auftretende Widerspruchsmarker „aber“ indiziert neben der Nichtübereinstimmung auf inhaltlicher Ebene auch die gleichzeitige „Beanspruchung des Rederechts“ auf der interaktionalen Ebene (Gruber 1996: 173). In Zeile 254 bis 256 lenkt Nelli mit „also MIR war das dann- mir war_s halt dann IRgendwann auch übertrieben- mit den BEIden.“ ein, bewertet in direktem Anschluss durch ,wobei ich die echt (.) TOLL: fand. wie synchron die ABgestimmt sind-“ (Z. 257-258) die Inszenierung zweier im Stück agierender Figuren positiv, wobei das Bezugsobjekt („mit den BEIden“, Z. 256) ihrer Bewertung vorerst vage bleibt und erst nach dem Entgegennehmen der Getränke und dem Entschluss, „nach DRAUßen“ (Z. 267) zu gehen mit „ich FINde die beiden:- die d d die (.) ZWILlinge- oder die die ZWILlinge.“ (Z. 273-275) von Nelli spezifiziert wird. Die beiden im Stück agierenden Figuren scheint Nelli einer erneuten Bewertung unterziehen zu wollen (,also WIE die das- mh wie die das MAchen:?“, Z. 279/281), wird aber von Wanja durch seine überlappend geäußerte und Nelli partiell zustimmende Bewertung ,ja: aber (.) das waren doch MEHR oder weniger- dasalso das WAR (.) ich- fand die hatten so:_n chaRAKter- (0.3) ja die ham das gut geMACHT-“ (Z. 280-286) unterbrochen. Seine positive Bewertung „ja die 
ham das gut geMACHT-“ (Z. 286) expandiert Wanja durch die rhetorische Frage „aber WA- (0.3) was- was ham die denn zur STOry beigetragen.“ (Z. 287-290) sowie durch die nachfolgende Bewertung „das war dann einfach nur so_n PAUsenclown.“ (Z. 291), der Nelli in Zeile 293-294 explizit und ohne jegliche Abschwächung widerspricht („nee:. das STIMMT nicht.“). Ihre Nichtübereinstimmung mit Wanjas Bewertung begründet Nelli durch ihre Beispielschilderung „die waren ja HANDlungsträger. (0.3) die ham ja ZWIschendurch immer- beRICHtetdit was jetz zum BEIspiel ähm:- (0.3) (äh)- mit den SCHIFfen passiert is. das war ja SCHON so a:ls ä:h- das war ja SCHON:- (0.6) so dass die SAchen: ä:hm:erZ̈̈HLT haben- die im HINtergrund pasSIERT sind auch- (0.6) ALso:-“ (Z. 296-319, siehe auch Beispiel 55, Kapitel 7.5), mit der sie sich gleichzeitig von Wanjas Äußerung distanziert („das war SCHON so“). Wanja reagiert daraufhin mit einem Nichtübereinstimmung ankündigenden „Aber-“ (Z. 321), bricht seine Äußerung jedoch ab und behandelt diese Bewertungssequenz der beiden Figuren im Stück nach einer längeren Pause mit dem (ironischen) Kommentar „wenigstens is der KAFfee gut.“ in Zeile 323 als abgeschlossen. Dass Wanja seine Widerspruch indizierende Äußerung abbricht und einen Themenwechsel einleitet, könnte darauf hinweisen, dass ihm weitere Einwände hinsichtlich Nellis Belehrung beziehungsweise Begründungen der von ihm eingenommenen Position nicht zugänglich sind.

Ähnlich wie im Gespräch zwischen Marco und Moritz ist auch bei Nelli und Wanja eine variierende Intensität der Nichtübereinstimmung zu beobachten: Während Wanja von Anfang an seine Bewertungen ohne jegliche Vorsichtsmaßnahme realisiert, markiert Nelli zu Beginn des Ausschnitts ihre mit Wanja partiell nicht übereinstimmende Bewertung eines Teils der Inszenierung durch Abschwächungsmarker als heikel und potenziell Face-bedrohend. Dies ändert sich allerdings, als es um die Bewertung von zwei im Stück agierenden Figuren geht, deren Performance Wanja negativ bewertet: Nelli widerspricht Wanjas Position ohne dies als Face-bedrohend zu markieren und begründet ihren Widerspruch, indem sie vage einen Teil der Inszenierung als Beispiel rekonstruiert („dass die SAchen: ä:hm:- erZÄHLT haben- die im HINtergrund pasSIERT sind auch“, Z. 315).

In Beispiel 99 nimmt Nelli mit ihrer impliziten Bewertung Bezug auf die von Wanja geäußerte Stellungnahme „also ich DENK- ich (.) ich denke wie geSAGT- ALso- (0.3) das stück auf !DREI! stunden AUSzudehnen- auch wenn da zwanzig minuten PAUse is- das trägt_s halt !ü!berHAUPT nich-“ (siehe Beispiel 98) und schließt sich dieser globalen Kritik des Theaterstücks an. 
Beispiel 99: Köln_14-06-15_Kaufmann_Gr.2

$\begin{array}{ll}328 \text { Nelli: } & \text { aber was ich in (.) in ERster linie auch- } \\ 329 & \text { tatsächlich FINde? } \\ 330 & \text { ist- } \\ 331 & (0.6) \\ 332 & \text { dass ich DAS f- } \\ 333 & \text { auch !NICH! FINde- } \\ 334 & \text { dass der INhalt das:- } \\ 335 & \text { das TRÄGT. }\end{array}$

Obwohl das Gespräch zwischen Wanja und Nelli von Episoden der Nichtübereinstimmung bezüglich einzelner Aspekte der Inszenierung durchzogen ist, so stimmen letztendlich - anders als im Gespräch zwischen Moritz und Marco doch beide Beteiligten in einer negativen Globalbewertung und dem Entschluss überein, den Theaterbesuch vorzeitig beenden zu wollen.

Auch im folgenden Ausschnitt aus dem Gespräch zwischen Susanne und Gudrun, die in ihrer positiven Globalbewertung des Theaterstücks Der Kaufmann von Venedig übereinstimmen, kommt es zu einer Episode der Nichtübereinstimmung hinsichtlich der Art der Inszenierung von Theaterstücken im Allgemeinen (klassisch vs. modern), die nicht aufgelöst wird, sondern nach einer kurzen Pause durch einen Themenwechsel von Grudrun als abgeschlossen behandelt und auch im weitern Gesprächsverlauf nicht noch einmal aufgegriffen wird.

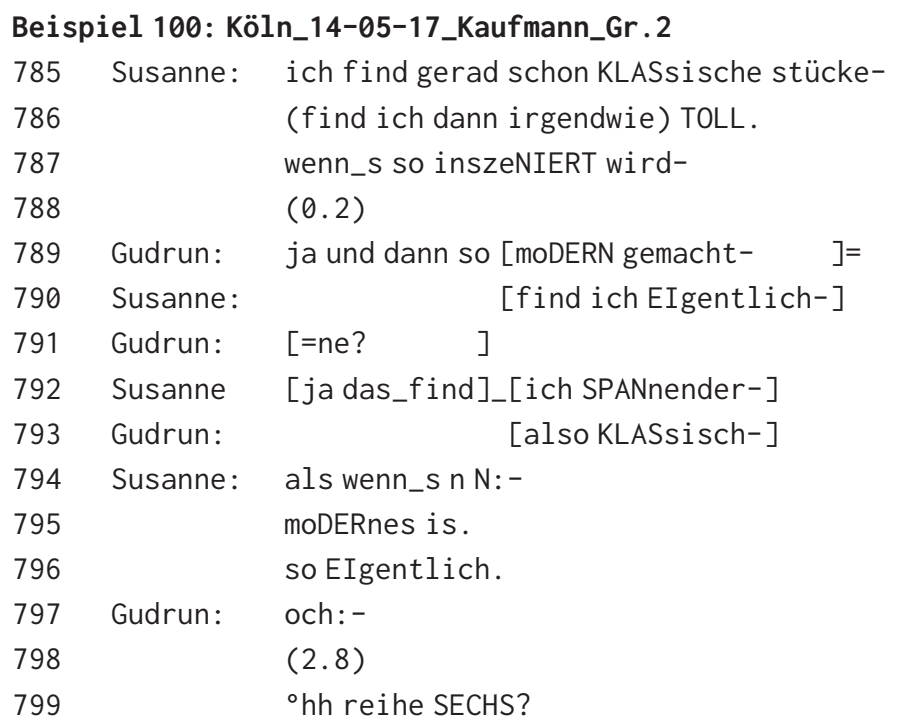


In Zeile 785 bis 787 äußert Susanne eine abgemilderte, positive Bewertung, mit der sie zum Ausdruck bringt, dass sie „gerad schon KLASsische stücke“, also „wenn_s so inszeNIERT wird“, toll findet. Gudrun quittiert diese Bewertung mit einem „ja“122 und ergänzt ihre Zustimmung um den Zusatz „und dann so moDERN gemacht“ (Z. 789), bevor sie mit dem Rückversicherungssignal „ne“ in Zeile 791 eine Bestätigung Susannes einfordert. Susanne fährt in Zeile 790 bis 792 - in Überlappung mit Gudrun - mit der erneut abgeschwächten Erläuterung ihrer ersten Bewertung klassisch inszenierter Stücke fort („find ich EIgentlich- ja das_find_ich SPANnender-"), während Gudrun (noch) davon ausgeht, dass Susanne ihr hinsichtlich der positiven Bewertung „moDERN gemachter“ Stücke zustimmt. In Zeile 793 beginnt Gudrun mit „also KLASsisch“ mit einer intonatorisch steigenden Äußerung, die sie allerdings, vermutlich aufgrund der in Überlappung von Susanne produzierten Äußerung ,ja das_find_ich SPANnender“ (Z. 792) abbricht. Gudrun quittiert Susannes Äußerung in Zeile 797 mit der Nichtübereinstimmung projizierenden Interjektion „och“ und initiiert einen aktuell relevanten Themenwechsel, indem sie sich nach einer Pause nach der Sitzreihe erkundigt (Z. 799). Es kommt zu keiner weiteren Aushandlung der Bewertungen.

Auch Gabriele und Cecilia kommen hinsichtlich der Bewertung der Gesänge im Theaterstück Der gute Mensch von Sezuan nicht überein. ${ }^{123}$ Während Gabriele die musikalische Darbietung „ganz toll“ findet, bezeichnet Cecilia diese als „komisch“ und „merkwürdig“. Entsprechend dem Vorgehen der beiden Beteiligten im vorangehenden Ausschnitt bleibt die Nichtübereinstimmung hinsichtlich der Bewertung eines Aspekts der Inszenierung bestehen und wird durch einen (abrupten) Themenwechsel von einer der beteiligten Gesprächspartnerinnen als abgeschlossen behandelt. Der nachfolgende Ausschnitt illustriert den gemeinsamen Austausch der Bewertungen von Gabriele und Cecilia mit Blick auf die Musik im Stück:

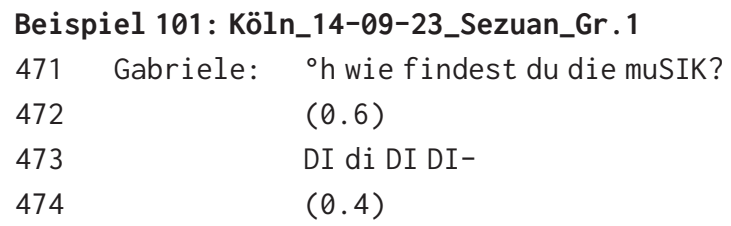

122 Auf Basis der Audioaufnahme wird paraverbal nicht deutlich, ob es sich bei dem von Gudrun geäußerten ,ja“ um eine Zustimmung in Bezug auf Susannes Bewertung handelt oder ob „ja“ in diesem Fall der Status eines Diskursmarkes zugeschrieben werden kann.

123 Ob Cecilia und Gabriele in der Globalbewertung des Thaterstücks übereinstimmen, lässt sich auf Basis des Gesprächs nicht eindeutig nachvollziehen, da im gesamten Gespräch keine Globalbewertung initiiert oder vorgenommen wird. 
475 Cecilia: EHRlich ge[sagt_n bisschen MERKwür]dig.

476 Gabriele: [DI di DI DI- ]

$(0.4)$

478

ich find es GANZ TOLL.

479 Cecilia: find_s die GUT?

480 Gabriele: also RICHtig TOLL find ich das. und äh mich würd ma intresSIERN wer diese musik (. ) ge $<<$ lachend $>$ MACHT hat $>$.

495 Gabriele: des fand ich GANZ TOLL.

496 Cecilia: FANDS_du?

497 Gabriele: GANZ TOLL fand ich das ja.

499 Cecilia: oKE:?

500 Gabriele: (.) WITzig.

501

502 TOLL finde.

508 Cecilia: hm.

510 Gabriele: du NICH?

$511 \quad(0.5)$

512 Cecilia: bin nich so RICHtig überzeugt. 


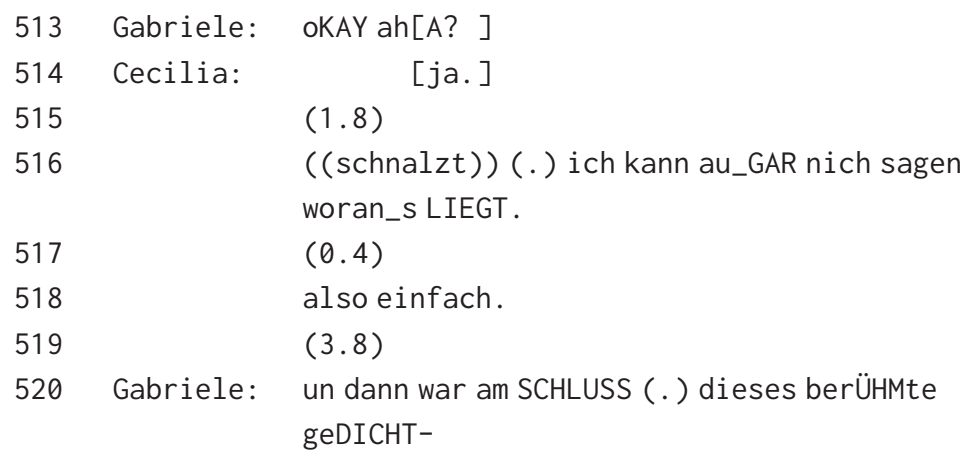

Wie bereits in Kapitel 8.1 (Beispiel 67) angeführt, initiiert Gabriele in Zeile 471 die Bewertungsinteraktion, indem sie ihre Gesprächspartnerin Cecilia zu einer bewertenden Stellungnahme der Musik im Theaterstück auffordert. Cecilia gibt an, die Musik „EHRlich gesagt_n bisschen MERKwürdig“ (Z. 475) zu finden. Der von Cecilia ihrer Stellungnahme vorangestellte Disclaimer „EHRlich gesagt“ könnte hier zum einen dazu dienen, ihre Position als potenziell heikel zu markieren sowie sich selbst von dieser zu distanzieren (siehe auch Beispiel 35, Kapitel 7.3), zum anderen projiziert Cecilia bereits eine mögliche Nichtübereinstimmung Gabrieles, die sich im weiteren Verlauf der Sequenz auch manifestiert. Gabriele rezitiert überlappend mit Cecilias Äußerung die Melodie der Musik im Stück (Z. 476) und bewertet dann die Musik mit ,ich find es GANZ TOLL.“ in Zeile 478 sehr positiv. Cecilia quittiert diese mit ihrem Eindruck nicht übereinstimmende Bewertung nicht mit einer weiteren Bewertung, sondern mit der (rhetorischen) Nachfrage „find_s die GUT?“ (Z. 479), woraufhin Gabriele ihrer Erstbewertung mit „also RICHtig TOLL find ich das.“ (Z. 480) nochmals Nachdruck verleiht. Auf diese zweite Bewertung Gabrieles folgt keine weitere Reaktion Cecilias. Stattdessen verkündet Gabriele, dass sie „ma intresSIERN“ würde, „wer diese musik (.) ge<<lachend>MACHT hat > (Z. 482). Nach mehreren kurzen Pausen und Zögern liefert Cecilia ab Zeile 488 einen ersten Erklärungsversuch für die Einschätzung der Musik im Stück und rekonstruiert die Reihenfolge des Einsetzens der verschiedenen Musikinstrumente. Das, was Cecilia als „KOmisch“ (Z. 488) empfunden hat, bewertet Gabriele in Zeile 495 nochmals als „GANZ TOLL“. Im Anschluss an Gabrieles Bewertung zeigt sich eine ähnliche Ausprägung wie bereits eingangs der Bewertungsinteraktion (Zeile 478 bis 480): Cecilia quittiert Gabrieles Bewertung mit der Nachfrage „FANDS_du?“ (Z. 496) und fordert diese damit zu einer erneuten Stellungnahme beziehungsweise Bestätigung ihrer Bewertung auf, die Gabriele dann mit „GANZ TOLL fand ich das ja.“ (Z. 497) auch liefert. Cecilia nimmt die wiederholte und betont positive Bewertung Gabrieles mit einem fragend intonierten „oKEE?“ (Z. 499) zur 
Kenntnis, schließt aber keine weiteren Ausführungen daran an. Gabriele hingegen steigt ab Zeile 500 mit „WITzig“ in eine Begründung ihrer positiven Bewertung ein (,weil ich die ä:hm- (0.8) die (.) TÖne in ihrer ABfolge w äh (.) GANZ GANZ TOLL finde.“, Z. 504-506). Auf Cecilias wenig Überzeugung anzeigendes „hm“ (Z. 508) hin fordert Gabriele mit ihrer Nachfrage „du NICH?“ in Zeile 510 einerseits eine Stellungnahme Cecilias ein, andererseits ist die Nachfrage ein Hinweis darauf, dass Gabriele Cecilias Reaktion als Indiz für Nichtübereinstimmung deutet. Die von Gabriele eingeforderte Stellungnahme beziehungsweise die Bestätigung der mit ihrer Nachfrage implizierten Deutung liefert Cecilia mit ihrer Äußerung „bin nich so RICHtig überzeugt.“(Z. 512). Gabriele nimmt dies mit „oKAY ahA?“ (Z. 513) zur Kenntnis, was Cecilia wiederum mit „ja“ (Z. 514) in Überlappung mit dem von Gabriele geäußerten Erkenntnisprozessmarker „ahA“ bestätigt. Nach einer kurzen Pause wechselt Cecilia auf eine metareflexive Ebene, führt aus, dass ihr (vorerst) keine Begründung ihrer Bewertung zugänglich ist (,((schnalzt)) (.) ich kann au_GAR nich sagen woran_s LIEGT. (0.4) also einfach.“, Z. 516-518) und markiert ihre Bewertung damit gleichzeitig als begründungsrelevant. Im Anschluss bricht sie ihre Äußerung ab. Gabriele leitet daraufhin mit „un dann war am SCHLUSS (.) dieses berÜHMte geDICHT-“ in Zeile 520 einen Themenwechsel ein und nimmt einen anderen im Theaterstück präsentierten Aspekt in den Blick. Dieser Themenwechsel dient möglicherweise - ähnlich wie in Beispiel 88 und Beispiel 95 - dazu, Cecilia durch die Fokussierung eines ihr durch ihre Rezeptionserfahrung zugänglichen Objekts (wieder) am Gespräch zu beteiligen. Die Bewertung der Musik im Stück wird im weiteren Gesprächsverlauf nicht nochmal aufgegriffen und somit von den Beteiligten als abgeschlossen behandelt.

\subsubsection{Aufgelöste lokale Nichtübereinstimmung bei globaler Übereinstimmung}

Neben den beiden vorangehend dargestellten Möglichkeiten, Nichtübereinstimmung im gesamten Gesprächsverlauf nicht aufzulösen und auch in der Globalbewertung des Theaterstücks nicht übereinzustimmen sowie - trotz übereinstimmender Globalbewertung des Theaterstücks - in einigen Bewertungsaspekten nicht übereinzustimmen, soll nachfolgend noch eine weitere Möglichkeit aufzeigt werden, die in den analysierten Gesprächen auftritt: Obwohl die Beteiligten in der Globalbewertung des Theaterstücks übereinstimmen, kann es zu dissenten Sequenzen kommen, in denen Nichtübereinstimmung aber durch Aushandlung und die Konzession eines der Beteiligten aufgelöst und in Übereinstimmung überführt wird. 
Dies geschieht beispielsweise im Gespräch ${ }^{124}$ zwischen Marina, Christine und Sven in der Pause des Theaterstücks Alle sieben Wellen. Sven initiiert in Zeile 099, indem er das Theaterstück global bewertet, eine neue Bewertungssequenz. Dem folgenden Ausschnitt geht eine Gesprächspassage voraus, in der Marina mit einer selbstinitiativen Erstbewertung des Stücks das Gespräch eröffnet (siehe Beispiel 72) und in der die Gruppe über die Buchvorlage zum Theaterstück spricht.

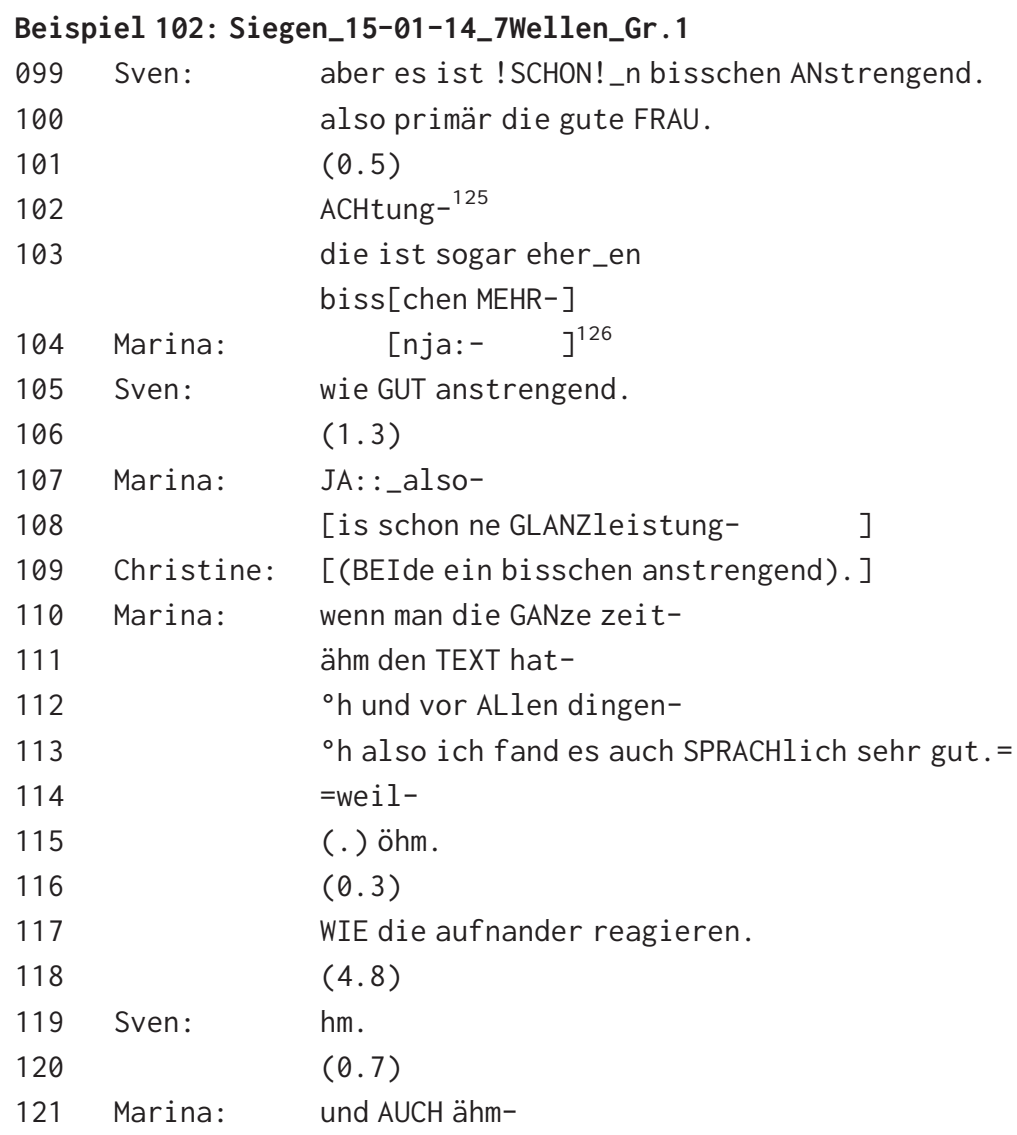

124 Siehe zu einer früheren Analyse dieses Beispiels Hrncal (2018: 265-268).

125 Aus der Audioaufnahme geht nicht deutlich hervor, ob diese Äußerung von Sven stammt. Da die Gruppe sich auf dem Weg vom Theatersaal ins Foyer befindet, ist davon auszugehen, dass sich die Äußerung auf die aktuelle Situation und nicht auf das aktuelle Gesprächsthema bezieht.

126 Dieses Rückmelde- beziehungsweise (Ver-)Zögerungssignal Marinas könnte vor dem Hintergrund ihrer Erläuterungen ab Zeile 105 bereits als Vorlaufelement auf ihre mit Svens Erstbewertung nicht übereinstimmende Zweitbewertung hinweisen. 


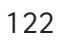

123

124

125

126

127

128

129

130

131

132

133 Marina:

134 Sven:

135 Marina:

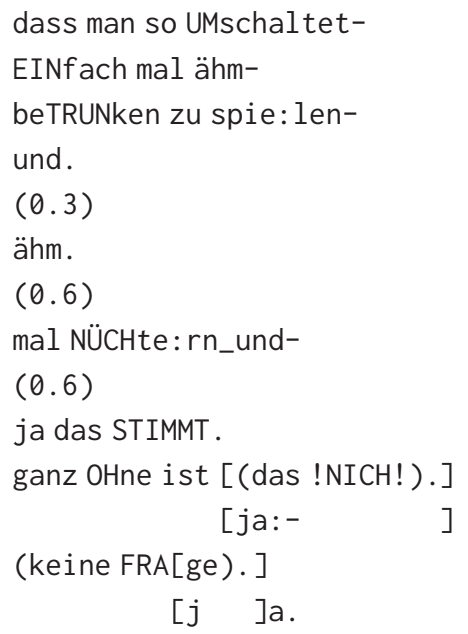

Sven realisiert mit seiner Äußerung „,aber es ist !SCHON!_n bisschen ANstrengend“ (Z. 099) eine abgeschwächte negative Globalbewertung des Theaterstücks. In Zeile 100 spezifiziert er das Bezugsobjekt seiner Bewertung durch den Verweis auf eine im Stück dargestellte Figur (,also primär die gute FRAU“, Z. 100), womit gleichzeitig ein Wechsel des Bezugsobjekts von der globalen zur ästhetisch-performativen Ebene einhergeht. Mit einer weiteren Bewertung, die Abschwächungsmarker und das positiv evaluierende Adjektiv „GUT“ als Intensivierer umfasst, stellt Sven fest, dass die im Stück dargestellte Frau ,sogar eher_en bisschen MEHR- wie GUT anstrengend“ (Z. 103-105) ist. Seine Bewertung begründet Sven allerdings nicht. Bereits in Zeile 104 indiziert Marina in Überlappung mit Sven durch ihre Äußerung Nichtübereinstimmung. In den Zeilen 107 bis 111 realisiert Marina mit „JA::_also- is schon ne GLANZleistung- wenn man die GANze zeit- ähm den TEXT hat“ eine positive Zweitbewertung, die an dieser Stelle bereits Widerspruch zur Äußerung von Sven projiziert. Christine stellt in Zeile 109 in Überlappung mit Marinas Zweitbewertung fest, dass „BEIde“ ${ }^{\text {“127 }}$ im Stück agierenden Figuren „ein bisschen anstrengend“ waren. Mit der weitere Erläuterungen projizierenden Floskel „und vor ALlen dingen-“ eingeleitet realisiert Marina eine weitere positive, subjektivierte und somit in ihrem Geltungsanspruch eingeschränkte Bewertung (, ${ }^{\circ} \mathrm{h}$ also ich fand es auch SPRACHlich sehr gut.“, Z. 112-113). Diese begründet sie nachfolgend durch den Verweis auf die Performance der Schauspieler (,weil- (.) öhm. (0.3) WIE die aufnander reagieren. (4.8) und AUCH ähm- dass man so UMschaltet- EINfach mal ähm- beTRUNken zu spie:len- und. (0.3) ähm. (0.6) mal NÜCHte:rn_und“,

127 Im gesamten Stück agieren lediglich zwei Figuren. 
Z. 114-129). Indem er Marinas Perspektive übernimmt, scheint Sven nach seiner Zustimmung in Zeile 131 (,ja das STIMMT“) mit seiner Feststellung ,ganz OHne ist (das !NICH!.) (keine FRAge.)“ (Z. 132-134) seine Erstbewertung in Zeile 099 bis 105 zu relativieren. Nach Marinas bestätigendem ,ja“ in Zeile 135 behandeln die am Gespräch Beteiligten die Bewertungssequenz als abgeschlossen.

Im nachfolgenden Ausschnitt ${ }^{128}$ nähern sich Holger und Adelheid trotz vorerst widersprüchlicher Bewertungen des Bühnenbilds des von ihnen besuchten Theaterstücks Der Kaufmann von Venedig am Ende der Bewertungssequenz in ihrer persönlichen Einschätzung doch noch an, da Holgers Argumentation Adelheid zu einer Konzession bewegt (Z. 139).

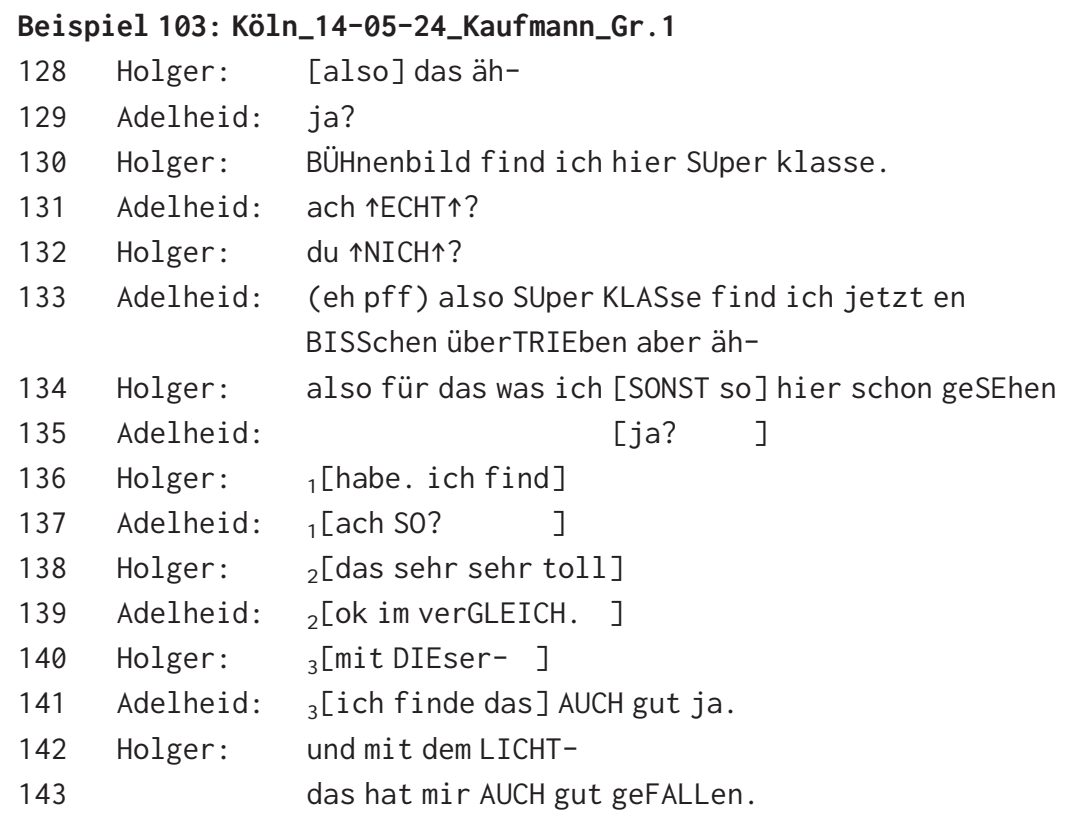

Mit dem Verweis „also das äh (.) BÜHnenbild find ich hier SUper klasse“ (Z. 128/ 130) nimmt Holger das Bühnenbild als Bezugsobjekt seiner Bewertung auf der ästhetisch-performativen Ebene in den Blick, hinsichtlich dessen er sich mittels der Gradpartikel „SUper“ und des evaluierenden Adjektivs „klasse“ explizit positiv positioniert. Adelheid reagiert mit der extrem steigend intonierten Nachfrage „ach 个ECHT个?““ (Z. 131) auf Holgers subjektivierte Erstbewertung („find ich“, Z. 130)

128 Siehe zu einer früheren Analyse dieses Beispiels Hrncal (2018: 260-263). 
und initiiert so eine Sequenzexpansion. Mittels der Interjektion ,ach“ und des Adjektivs „ECHT“ äußert Adelheid ihre Verwunderung und setzt eine Reaktion Holgers relevant. Holgers ebenfalls extrem steigend intonierte, geschlossene Nachfrage „du 个NICH个?““ (Z. 132) indiziert, dass er Adelheids Äußerung in Zeile 131 den Status eines Nichtübereinstimmung projizierenden Vorlaufelements zuschreibt. Außerdem setzt er durch seine Nachfrage die Beantwortung respektive Bestätigung seiner Frage durch Adelheid relevant. Nach einem Zögern („(eh pff)“) liefert Adelheid in Zeile 133 die Antwort, in der sie Holgers Bewertung (,SUper KLASse“) aufgreift und als „überTRIEben“ einstuft. Indem sie ihre Antwort in ihrem Geltungsbereich einschränkt (,find ich“) und abschwächt (,en BISschen“), markiert sie diese als potenziell heikel. Weitere, mit „aber (äh)“ projizierte Ausführungen schließt Adelheid nicht ab. Es kommt daraufhin zu einer Rechtfertigung Holgers, in der er seine Bewertung durch den Vergleich mit anderen Theaterstücken begründet (siehe auch Beispiel 58, Kapitel 7.5), die er „SONST so“ am aktuellen Spielort „schon geSEhen“ hat (Z. 134-136). Mit seiner Begründung verweist Holger implizit auf Wertmaßstäbe, die seiner positiven Bewertung vermutlich zugrunde liegen, und verändert so den Geltungsbereich (vgl. Auer/Uhmann 1982: 22) der von Adelheid monierten Bewertung. Seine in Zeile 136 begonnene und subjektivierte explizit positive Bewertung („toll“), mit der er scheinbar ein weiteres Bezugsobjekt („mit DIEser“) auf der ästhetischperformativen Ebene fokussiert, bricht er in Zeile 140 ab. Nach Adelheids Äußerung des Erkenntnisprozessmarkers (vgl. Imo 2009) „ach SO“ in Zeile 137 verbalisiert sie mit der Reziprozitätsdemonstration (vgl. Kallmeyer 1979: 93) „ok im verGLEICH“ den Wertmaßstab, den Holger seiner Erstbewertung implizit zugrunde gelegt hat. Im Gegensatz zu der von ihr als übertrieben eingestuften Erstbewertung Holgers lenkt Adelheid im Anschluss an Holgers Argumentation beziehungsweise Begründung seiner Bewertung ein, und äußert, dass sie das Bühnenbild „AUCH gut“ (Z. 141) findet. Im Anschluss an Adelheids Konzession fährt Holger mit einer neuen Erstbewertung fort, mit der er die Bühnenausleuchtung explizit positiv evaluiert (,und mit dem LICHT- das hat mir AUCH gut geFALlen“, Z. 142-143). Diese weitere Bewertung Holgers wird von den anderen Gesprächsteilnehmerinnen jedoch (vorerst) ${ }^{129}$ nicht aufgegriffen. In diesem Ausschnitt wird deutlich, wie bei einer anfänglichen Nichtübereinstimmung durch das Argumentieren für beziehungsweise das Begründen einer ersten

129 Der von Holger angesprochene Einzelaspekt (,das mit dem LICHT“) wird im weiteren Gesprächsverlauf von Adelheid nach einer minimalen Pause aufgegriffen, aber keiner Bewertung unterzogen. Stattdessen nimmt sie mit der einen Kontrast zu vorangehend Gesagtem indizierenden positiven Bewertung ,aber wer doch SUper ist sind diese beiden VÖgel oder“ im Stück agierende Figuren in den Fokus. 
Bewertung diese doch noch zustimmungsfähig gemacht und Nichtübereinstimmung von den Beteiligten interaktiv ausgehandelt werden kann.

\subsubsection{Ergebnisse}

Die in Kapitel 8.3 angeführten Gesprächsausschnitte illustrieren exemplarisch mögliche Formen nichtübereinstimmender Bewertungen und geben Aufschluss darüber, wie die Beteiligten Nichtübereinstimmung aushandeln. Insgesamt zeichnen sich in den in Kapitel 8.3 angeführten Beispielen drei Formen der Nichtübereinstimmung ab: Im ersten Fall stimmen die Beteiligten sowohl in ihrer Globalbewertung des Theaterstücks als auch bezüglich verschiedener Aspekte auf der ästhetisch-performativen Ebene des Theaterstücks nicht überein. Die Nichtübereinstimmung der Bewertungen wird weder hinsichtlich der Teilaspekte noch hinsichtlich des gesamten Stücks aufgelöst. Im zweiten Fall stimmen die Beteiligten zwar in der Gesamtbewertung des Theaterstücks überein, aber nicht in der Bewertung von Einzelaspekten auf der ästhetisch-performativen Ebene des Stücks. Liegt im dritten Fall eine Nichtübereinstimmung hinsichtlich der Bewertung einzelner Aspekte des Stücks bei gleichzeitiger Übereinstimmung in der Globalbewertung des Stücks vor, so gehen die Beteiligten auf zweierlei Weise damit um: Entweder versuchen die Erstbewertenden die Zweitbewertenden durch weitere Argumente doch noch von ihrer Bewertung zu überzeugen, was zu einer Aushandlung im Rahmen von Nichtübereinstimmung führen kann, in deren Zuge (lokale) Nichtübereinstimmung aufgelöst und in Übereinstimmung überführt wird. Andererseits können die Beteiligten auf ihrer jeweiligen Bewertung beharren, ohne sich in ihren Standpunkten anzunähern, was wiederum bedeutet, dass die vorliegende (lokale) Nichtübereinstimmung nicht aufgelöst wird und Episoden der Nichtübereinstimmung „durch Sprecherund Themenwechsel [enden]“ (Gruber 1993: 14) können. Auch hier bietet der gesellige Kontext neues Themenmaterial und kann so eine Entlastungsfunktion übernehmen.

\section{Dissens- und Präferenzmanagement}

Nichtübereinstimmung in den angeführten Beispielen kann in einer Perspektivendivergenz der Beteiligten hinsichtlich der ästhetischen Qualität von Einzelaspekten oder der gesamten Inszenierung des Theaterstücks begründet sein. Ob Übereinstimmung oder Nichtübereinstimmung präferiert wird (vgl. dazu auch Kotthoff 1993 und Lerner 1996), und wie die Sprecher mit einer durch Nichtübereinstimmung hervorgerufenen Face-Bedrohung umgehen (indem sie beispiels- 
weise ihre Bewertung beziehungsweise Positionierung als potenziell heikel markieren), kann anhand der Art und Weise nachgezeichnet werden, wie Nichtübereinstimmung von den Beteiligten im Gespräch eingeleitet wird. Nichtübereinstimmung kann dynamisch und in unterschiedlichen Intensitätsgraden im Laufe eines Gesprächs oder im Laufe einer dissenten Sequenz zwischen einer abgeschwächten und einer starken Ausprägung ohne jegliche Abschwächung variieren: Partieller Widerspruch (,ja aber“), Disfluenzmarker wie Vokaldehnungen oder Pausen sowie Unschärfemarkierungen („ein bisschen“, „irgendwie“ usw.) weisen auf moderaten Dissens hin, der innerhalb einer Sequenz auftritt und bearbeitet wird sowie „Aktivitäten der Renormalisierung“, das heißt „Signale des Einlenkens und Verständnisses“ (Kallmeyer 1979: 90) enthält, die zu Übereinstimmung zurückführen. Direkt hervorgebrachte Nichtübereinstimmung, die keine Vorlaufelemente sowie Mittel zur Abschwächung beinhaltet, sich über mehrere Sequenzen bis hin zur gesamten Gesprächslänge erstreckt und keine „Aktivitäten der Renormalisierung“ beinhaltet, deutet hingegen auf verschärften ${ }^{130}$ und unauflösbaren Dissens hin.

\section{Wissensmanagement}

Mit der Bearbeitung und dem Management von Nichtübereinstimmung ist auch die Aushandlung der Wissensstatus der Interagierenden (vgl. Deppermann 2015) verbunden. Dabei wird in den oben angeführten Beispielen vor allem die Dimension des Wissenszugangs (epistemic access) und die Dimension des Wissensvorrangs (epistemic primacy) (vgl. Stivers/Mondada/Steensig 2011; siehe auch Kapitel 2.3.3) von den Sprechern relevant gesetzt. Hinweise auf eine Relevantsetzung des Wissenszugangs geben Verweise auf im Stück präsentierte Inhalte, wie beispielsweise Nellis Rekonstruktion „dit was jetz zum BEIspiel ähm:- (äh)- mit den SCHIFfen passiert is. das war ja SCHON so a:ls ä:h- (. . .) das war ja SCHON:so dass die SAchen: ä:hm:- erZÄHLT haben- die im HINtergrund pasSIERT sind auch-“ (siehe Beispiel 98) oder Marinas Rekonstruktion „WIE die aufnander reagieren. dass man so UMschaltet- EINfach mal ähm- beTRUNken zu spie:len- und. ähm. mal NÜCHte:rn_und-“, mit der jeweils eine nicht übereinstimmende Bewertung begründet wird (siehe Beispiel 102). Eine Relevantsetzung des Wissensvorrangs wird beispielsweise durch eine Selbstpositionierung als relativer Laie und eine Fremdpositionierung als Experte (, ${ }^{\circ}$ hh okee sowas fällt mir zum beispiel gar nich AUF.= =aber du bist JA:- na DU:- als regisSEUR? MERKST es wahrscheinlich au einfach mehr-“, siehe Beispiel 93) oder durch den Verweis auf persönliche Erfahrungen indiziert (,also für das was ich SONST so hier schon geSEhen habe.“,

130 Vergleiche dazu auch Kotthoff (1993: 200-201). 
siehe Beispiel 103). Auch die teilweise als Begründungen von den Beteiligten angebrachten Belehrungen sprechen dafür, dass es unter anderem darum geht, Wissen auszutauschen, Wissensgefälle $\mathrm{zu}$ beheben und voneinander $\mathrm{zu}$ lernen.

\section{Deeskalationsressourcen}

Trotz der oben geschilderten Phasen der Nichtübereinstimmung oder sogar das gesamte Gespräch durchziehende Nichtübereinstimmung kommt es in keinem dieser Gespräche zu Streit, etwa in „einer Abfolge emotionaler Reaktionen und Gegenreaktionen“ oder gar der Erweiterung der Nichtübereinstimmung „vom inhaltlichen auf den persönlichen Bereich“ (Gruber 1993: 8, Herv. im Original). Dies ist als Zeichen für den kooperativen Charakter der Gespräche zu interpretieren. Obwohl die Sozialform der Kunstkommunikation nach außen exklusiv und sozial distinguierend ist (vgl. Müller/Kluwe 2012), geht es innerhalb der Gespräche, das haben die bisher analysierten Beispiele gezeigt, auch darum, Wissensasymmetrien zu beheben und einen in der Praxis der Kunstkommunikation möglicherweise relativ unerfahrenen Gesprächspartner an eben diese Praxis heranzuführen und dessen Beteiligung am Gespräch trotz eventuell unzureichender Kompetenz zu ermöglichen. Aufgrund dieser Beobachtung ist davon auszugehen, dass die Beteiligten neben defensivem Face-Work (zum Beispiel durch die Abschwächung ihrer Bewertungen) durch Maßnahmen zur Beteiligung ihrer Gesprächspartner auch kooperatives Face-Work betreiben (vgl. Goffmann 1967: 27), um nicht nur ihr eigenes Face, sondern auch das ihres Gegenübers zu schützen.

Neben dem kooperativen Charakter könnten auch die situativen Umstände der Pausengespräche dafür ausschlaggebend sein, dass es zu keiner Eskalation kommt. Denn im Gegensatz zu privaten Alltagsgesprächen, in denen es durchaus zu Streit und zur Eskalation kommen kann (vgl. Keppler 1994), finden die Foyergespräche zwar innerhalb einer privaten Gruppe statt, aber nicht in der vertrauten und gegenüber der Öffentlichkeit abgeschirmten Umgebung des eigenen Zuhause, sondern in einer öffentlich zugänglichen Spielstätte. Ein Streit würde also nicht in der „Privatsphäre“ der Gesprächsgruppe bleiben (vgl. Keppler 1994), sondern könnte von den ebenfalls im Foyer anwesenden Personen beobachtet werden und unmittelbare soziale Konsequenzen haben (vgl. auch Habscheid/Linz 2018: 3). Auch der zeitliche Rahmen der Gespräche spielt möglicherweise eine Rolle, da den Gesprächspartnern nur ein begrenzter Zeitrahmen nämlich die Dauer der Pause zwischen den beiden Teilen des Theaterstücks oder zwischen dem Theaterstück und dem sich daran anschließenden Publikumsgespräch - zur Verfügung steht. Und drittens ist der gesellige Charakter der Pausengespräche zu nennen: Sowohl die Tatsache, dass Bewertungen beispielsweise auch in Form theatraler Reinszenierungen realisiert werden als auch der in eini- 
gen Gesprächen zu beobachtende humorvolle Umgang mit der Situation des Pausengesprächs sprechen dafür, dass es in den Gesprächen auch darum geht, sich zu vergnügen und am emotionalen Erleben der anderen Teil zu haben.

\subsection{Ausstiege aus Bewertungsinteraktionen}

Sowohl die Analyse möglicher Verläufe von Bewertungsinteraktionen als auch die Analyse der sequenziellen Aushandlung nicht übereinstimmender Bewertungen haben bereits gezeigt, dass die Beteiligten jederzeit selbst den Ausstieg aus einer Bewertungsinteraktion, beispielsweise durch einen Themenwechsel, initiieren können. Da die Pausengespräche aufgrund ihres semi-institutionellen Charakters (siehe Kapitel 2.3.2) in einem zeitlich begrenzten Rahmen stattfinden, kann das nicht immer exakt vorhersehbare Ende der Pause, eingeleitet durch ein akustisches Signal, ${ }^{131}$ für die Beteiligten überraschend sein (vgl. Habscheid/Linz 2018: 4-5). Anhand der im Folgenden analysierten Gesprächsausschnitte erfolgt eine Auseinandersetzung mit der Frage, wie die Beteiligten selbst Ausstiege aus Bewertungsinteraktionen vorbereiten und vollziehen und inwiefern sie das akustische Signal und das damit markierte Pausenende in ihren Gesprächen thematisieren beziehungsweise inwiefern das Signal zu einem Abbruch der Bewertungsinteraktion führen kann.

\subsubsection{Relevantsetzung des Ausstiegs durch ein Signal von außen}

Im folgenden Beispiel 104 führt das akustische Signal zur Markierung des Pausenendes zu einem Abbruch einer Begründungsaushandlung zwischen Susanne und Dina im Anschluss an eine Bewertung, indem Susanne das Pausenende durch ihren Verweis „glaub ich muss_ma REIN.“ (Z. 357) unmittelbar relevant setzt:

\section{Beispiel 104: Köln_14-09-25_DieLücke_Gr.3}

311 Susanne: aber des mit dem BüHnenbild des find ich schön dass dir_s gefällt.= =ich find_s nämlich AUCH klasse.

314 Dina: ich mag das GERne.

131 Im Falle des Theaterstücks Der gute Mensch von Sezuan läutet kein akustisches Signal das Ende der Pause zwischen den Teilen des Theaterstücks ein, sondern der zweite Teil des Stücks beginnt im Theaterfoyer (siehe Kapitel 6.6). 
315

316

317

318

319

320

321

322

323

324

325

326

327

328

329

330 Susanne:

331 Dina:

332

333

334

335

336

337

338

339

340

341

342

344

345

346

347

348

349

Dina:

Susanne:

Dina:

Susanne: ich mag das SEHR gerne.

wenn das (. ) BÜHnenbild irgendwie auch so_n

BISSchen-

(0.4)

moderniTÄT zeigt.

weißte wenn des NICH so_n.

(1.4)

KONservatives bühnenbild is das man irgendwie erwArten kÖnnte.

(1.0)

zum beispiel MOCHT ich diese EIne-

(0.9)

aufführung die wir in der Oper gesehn ham die so auf GANZ modDERN gemacht war-

(0.6)

Susanne: [ja. ]

Dina: [die m]ocht ich toTAL.

wo plötzlich ASterix un Obelix durch die

$\mathrm{b}$ [ühne. es war klasse.

]

[jaja (.) un des WILDschwein] und so (. ) ja.

es war KLASse.

(0.6)

ja.

(2.0)

ja wo einfach was überRAschendes is und-

(0.9)

ja wo man SICH auch-

$(0.4)$

weißte wenn ALles so:-

$(0.4)$

Susanne: also da [war_s halt GUT weil des so_n spiel]

[wie in_nem FILM oder so.

mit so kliSCHEES war bei diesem bühnenbild da (.)

mit dem ASterix [(und)] ((unverständlich))

$[\mathrm{mi}-\quad]$

ach SO.

i ich mei[n DER- ]

[ja. ]

$(0.3)$

un DA war_s ja jetz- 
351 Dina:

352 Susanne:

353

354

355

356

357

358

359

360 des warn [ja lauter offene S

[wegen des ((unverständlich))]

eigentlich.

also bis auf das ((unverständlich)) das jetz sehr

(. ) (PLAStisch/KLASsisch) waranSONSten hast du ja-

(0.9)

ganz WEnig eigentlich geHABT.

Kommentar: ((Eine Glocke wird geläutet, 5.0 Sek. ))

Dina: un die STRAße.

Susanne: glaub ich muss_ma REIN.

Dina: ja.

(2.5)

$\mathrm{Zu}$ Beginn des Ausschnitts (Z. 311) nimmt Susanne auf eine vorangegangene Bewertung Dinas Bezug, indem sie die Tatsache positiv bewertet, dass Dina das Bühnenbild des von beiden besuchten Theaterstücks Die Lücke gefällt. Susanne schließt an diese Bezugnahme in Form einer Bewertung eine weitere positive Bewertung, diesmal hinsichtlich des Bühnenbilds, an (Z. 312), mit der sie sich Dinas Bewertung anschließt. Nach einer kurzen Pause äußert Dina eine Geschmacksbekundung (Z. 314, siehe auch Beispiel 59, Kapitel 7.5), mit der sie ihre positive Bewertung des Bühnenbilds begründet und in deren Anschluss sie ihrer Bewertung zugrunde gelegte Wertmaßstäbe offenlegt (316-321). Ab Zeile 323 zieht sie das Bühnenbild einer von ihr und Susanne in der Vergangenheit rezipierten Theateraufführung als Beispiel und Vergleich heran. Darauf folgt eine ko-produzierte Rekonstruktion des Bühnenbilds beziehungsweise der Inszenierung (Z. 329-339), die in einer Bewertung Susannes mit Bezug auf das Bühnenbild des in der Vergangenheit von ihr und Dina besuchten Theaterstücks mündet (Z. 341). Im Anschluss an diese Bewertung nimmt Susanne mit „un DA war_s ja jetz-des warn ja lauter offene STELlen eigentlich. also bis auf das ((unverständlich)) das jetz sehr (.) (PLAStisch/KLASsisch) war- anSONSten hast du ja- (0.9) ganz WEnig eigentlich geHABT.“ (Z. 349-355) vermutlich Bezug auf den unmittelbar zuvor von ihnen rezipierten ersten Teil des Theaterstücks Die Lücke und kontrastiert dieses mit dem vorangehend rekonstruierten Bühnenbild aus einer vergangenen Theateraufführung. In direktem Anschluss an Susannes Kontrastierung werden die beiden in Zeile 356 durch ein akustisches Signal, das das Pausenende markiert, unterbrochen. Während Dina in Zeile 357 mit der aktuellen Themenbearbeitung fortfährt und einen neuen Aspekt, vermutlich des Bühnenbilds, anspricht, reagiert Susanne mit den Worten „glaub ich muss_ma REIN.“ (Z. 358) unmittelbar und explizit auf 
das akustische Signal. Dieser Verweis führt dazu, dass Dina ihren in Zeile 357 begonnenen Turn abbricht. Die beiden Gesprächspartnerinnen kehren in den Theatersaal zurück und das durch die Pause unterbrochene Thema wird von den beiden im weiteren Gesprächsverlauf nicht nochmal aufgegriffen. Der Abschluss der Bewertungsinteraktion bleibt in diesem Beispiel offen und wird nicht interaktiv von den beiden Beteiligten zu Ende geführt.

In Ausschnitt 105 verweist Cornelia im Anschluss an eine side sequence (vgl. Jefferson 1972), in der sie Irmgard ein Foto auf ihrem Handy zeigt, durch ihre lokal sensitive Frage „(is) schon WEIter- oder is das schon WEItergegangen?“ (Z. 340-341) auf den zeitlich begrenzten Rahmen des Pausengesprächs und den Beginn des sich an die Pause anschließenden Publikumsgesprächs:

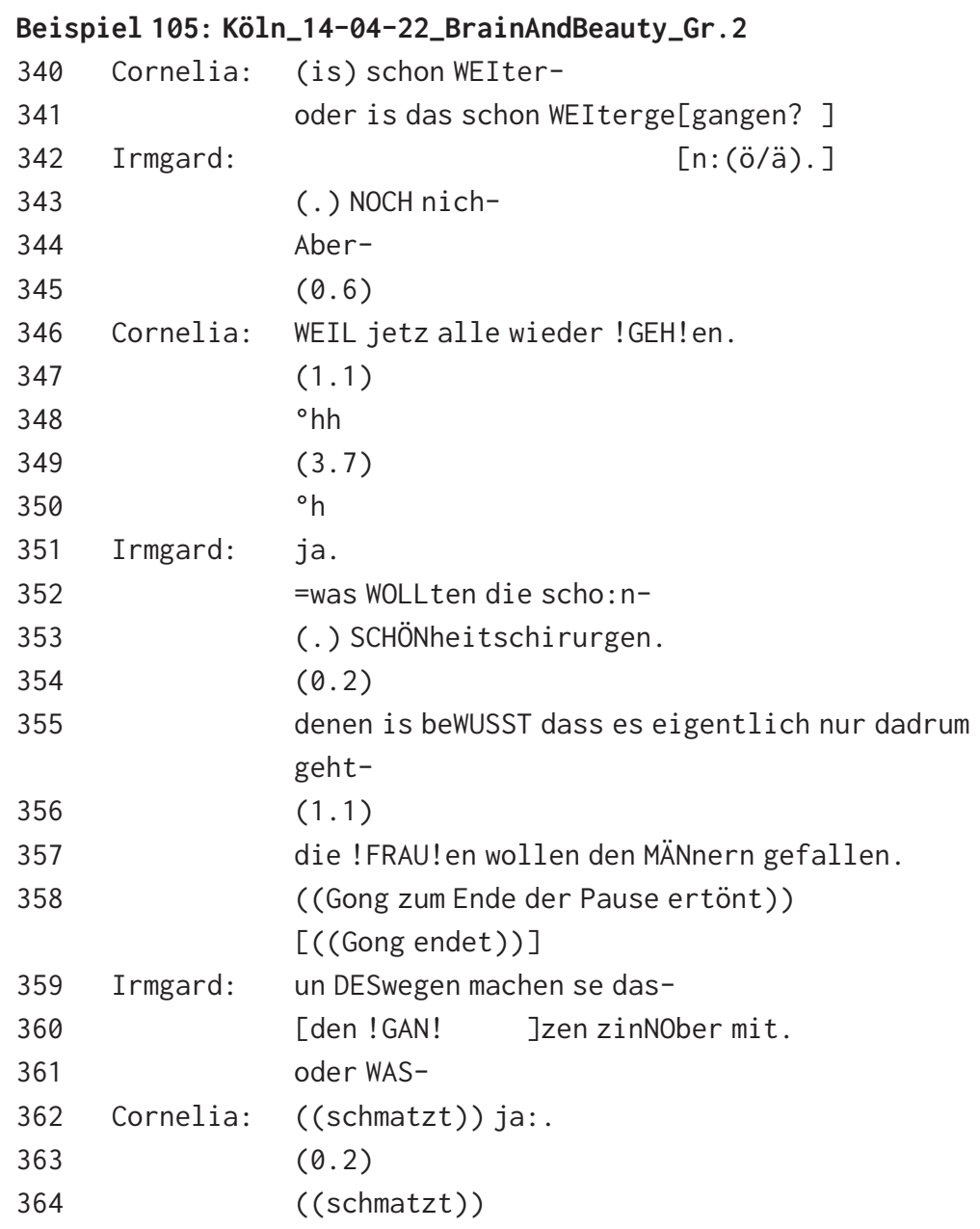


365

366

367

368

369

370

371

372

373

374

375

376

377

378

379

380

381

382

383
(3.1)

((schmatzt)) jetz hat_s aber geKLINGelt. jetz geht_s aber SCHON weiter.

$(0.7)$

Irmgard: ja: .

aber jetz ! TRINK! ich meinen KAFfee au noch aus.

Cornelia: ((lacht))

Irmgard: oder kann_man den mit RÜbernehmen .

((unverständlich))

Kellner: ((erlaubt das Mitnehmen der Getränke))

Irmgard: [oKAY- ]

Cornelia: $[(($ lacht $))]$

Irmgard: DANkeschön.

$(0.8)$

wir können auch RECHTS rum gehen.

$=$ ne?

(1.1)

Cornelia: ((schmatzt)) ja-

(8.5)

Nachdem Irmgard Cornelias Frage verneint hat, nennt Cornelia in Zeile 346 den Grund ihrer Nachfrage beziehungsweise Vermutung. Irmgard reagiert nicht unmittelbar auf Cornelias Äußerung, sondern greift nach einer längeren Pause die vor der side sequence im Anschluss an eine Bewertung initiierte Deutungsaushandlung wieder auf (Z. 351-352). Das akustische Signal zum Pausenende ertönt, während Irmgard die Funktion der im Stück inszenierten Schönheitschirurgen und deren Absicht zu deuten versucht. Im Gegensatz zu Susanne in Beispiel 104 setzt Irmgard nicht unmittelbar im Anschluss an das Signal den Ausstieg aus der Deutungssequenz relevant, sondern schließt ihre Deutung ab, indem sie in Zeile 361 eine Reaktion Cornelias relevant setzt. Cornelia bestätigt Irmgards Deutung (Z. 632), initiiert keine weitere Expansion der Deutungssequenz und behandelt diese somit als abgeschlossen. Nach einer Pause verweist sie mit ,jetz hat_s aber geKLINGelt. jetz geht_s aber SCHON weiter“ (Z. 366-367) auf das Ende der Pause. Irmgards Reaktion „ja:. aber“ (Z. 369-370) weist darauf hin, dass sie Cornelias Verweis auf das Pausenende als Aufforderung zur Rückkehr in den Theatersaal deutet. Nachdem Irmgard sich versichert hat, dass sie ihr Getränk mitnehmen darf, kehren die beiden Gesprächspartnerinnen in den Theatersaal zurück. Irmgards kommuniziertes Bedürfnis, zuerst ihren Kaffee austrinken zu wollen, gibt in diesem Beispiel einen Hinweis darauf, dass die Theaterpause auch tatsächlich als Pause von den Beteiligten wahrgenommen und zur Entspannung genutzt 
wird. Auch in diesem Ausschnitt wird der von einer Gesprächsteilnehmerin initiierte Deutungsversuch möglicherweise aufgrund der Äußerungen Cornelias, die eine Rückkehr in den Theatersaal relevant setzen, nicht weiter expandiert.

Im nächsten Ausschnitt wird das Pausenende nicht durch eine Pausenklingel markiert, sondern der zweite Teil des Theaterstücks wird noch im Foyer begonnen, was bei Gudrun zu Verunsicherung führt:

\section{Beispiel 106: Köln_14-05-17_Kaufmann_Gr.2}

1027 Susanne: in diesem NEUen theater mitten-

1028

1029

1030

1031

1032

1033

1034

1035

1036

1037

1038

1039

1040

1041

1042

1043

$((\ldots))$

in HAMM-

da war_ich AUCH neulich-

und da ham_sie die ganze zeit geGESsen?

(0.4)

und die HAM dann wirklich-

äh WIRKlich gegessen.

und ich hatte [!SO_N! HuNger-]

Gudrun:

[WIE die-

wie.

die SCHAUspieler-

Susanne: ja.

${ }^{\circ} \mathrm{h}$ die SAßen.

Gudrun: ((lacht))

Susanne: da gab_s LANge szenen-

wo die zusammen am TISCH saßen?

$(0.4)$

1061 Gudrun: hast du MAL gescha-

1062

1063

1064

1065

1066

1067

1068

geSCHAUspielt irgendwie?

AUßer in_der-

in_der SCHU[le?]

Susanne:

[j ]a.

$(0.3)$

Gudrun: $\quad<$ erstaunt $>$ JA_a>?

1069

viel-

1070

SEHR viel.

1071

1072

ich $\mathrm{HAB}$ jetzt grad-

1073

guck_mal da kommt dein LIEBling-

1074

$(0.3)$ 


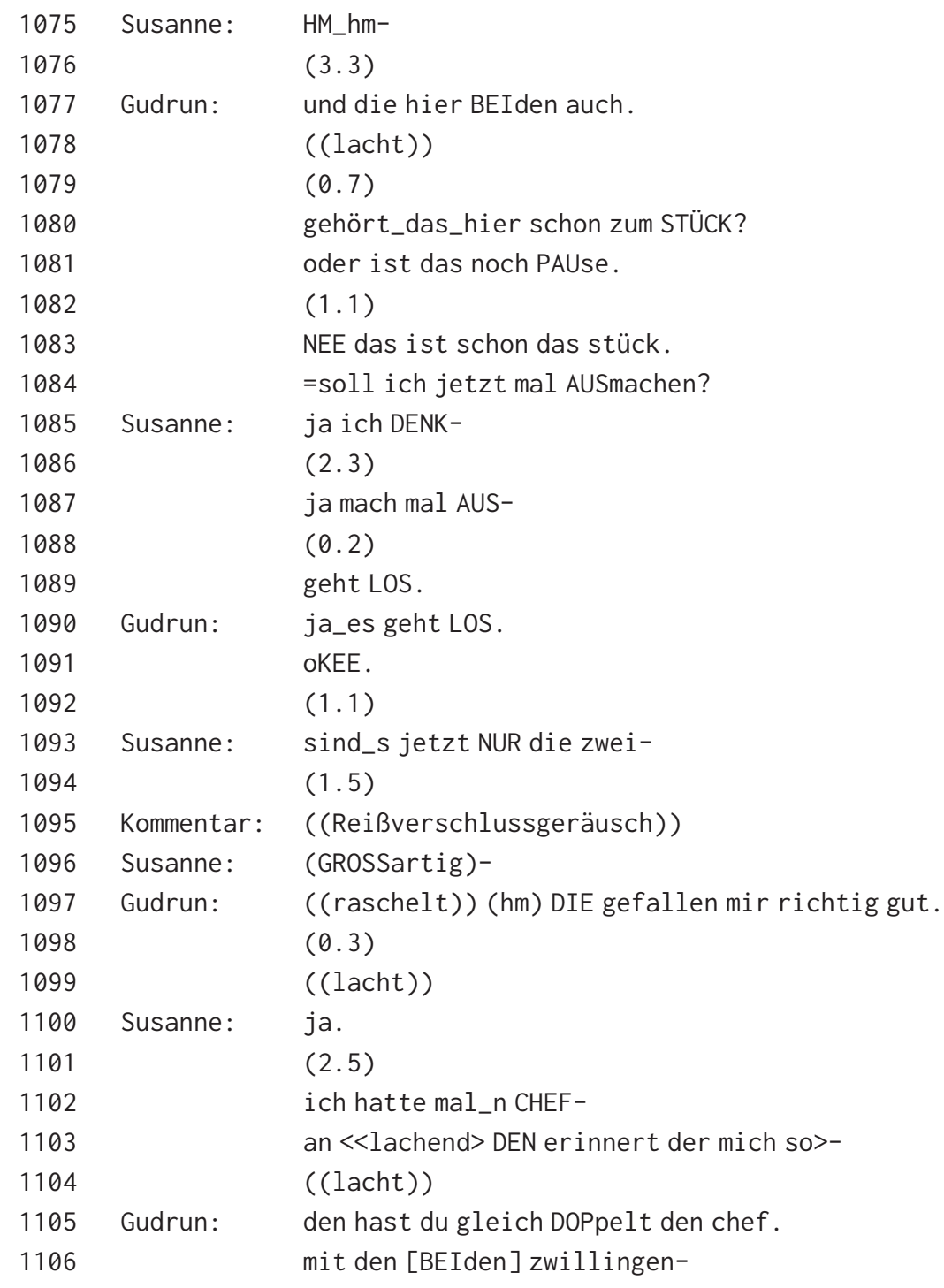

Nachdem Susanne zu Beginn des Ausschnitts Rezeptionserfahrungen aus einem anderen Theaterstück geschildert hat, initiiert Gudrun in Zeile 1061 einen Austausch über bisherige Schauspielerfahrungen. Ihre Ausführungen in Zeile 1072 bricht sie ab und lenkt Susannes Aufmerksamkeit mit „guck_mal da kommt dein LIEBling“ (Z. 1073) auf einen in den gemeinsamen Wahrnehmungsraum im Theaterfoyer tretenden Schauspieler aus dem Theaterstück. Als zwei weitere Schauspieler hinzukommen (,und die hier BEIden auch.“, Z. 1077), vergewissert 
sich Gudrun, ob mit dem Auftreten der Schauspieler im Foyer das Theaterstück bereits weitergeführt wird („gehört_das_hier schon zum STÜCK?“, Z. 1080) oder es noch ein Teil der Theaterpause ist („oder ist das noch PAUse.“, Z. 1081). Nach einer Pause beantwortet sie dann mit „NEE das ist schon das stück“ (Z. 1083) selbst ihre Nachfrage und vergewissert sich bei Susanne, ob das Aufnahmegerät nun ausgeschaltet werden soll (Z. 1084). Susanne bejaht dies und beide Gesprächspartnerinnen versichern sich, dass das Stück nun weitergeht (Z. 1089-1090). Im Gegensatz zu den Beispielen 104 und 105, in denen durch das Markieren des Pausenendes die aktuell bearbeiteten Themen abgebrochen werden, initiiert Susanne lokal sensitiv durch den Verweis auf die beiden Schauspieler (Z. 1093) und ihre daran angeschlossene Bewertung „(GROSSartig)“ (Z. 1096) eine neue Bewertungssequenz, an die Gudrun mit einer subjektivierten und im Vergleich zu Susanne deskalierten Zweitbewertung anschließt, die Susanne in einem dritten Schritt wiederum bestätigt (Z. 1100). Nach einer Pause stellt Susanne mit ihrem Verweis auf beziehungsweise den Vergleich ihres ehemaligen Chefs (Z. 1102) mit den beiden im Stück dargestellten Figuren einen Bezug zu ihrer eigenen Alltagswirklichkeit her, den Gudrun mit ihrem ironischen Kommentar (Z. 1105-1106) aufgreift. In diesem Beispiel führt das für die Beteiligten überraschende Weiterführen des Theaterstücks im Foyer während der Pause zuerst zu Verunsicherung, die eine gegenseitige Verständigung über das sich im Foyer Ereignende bedingt. Entgegen eines $\mathrm{zu}$ erwartenden Abbruchs des Gesprächs kommt es allerdings zu einer lokal sensitiven Initiierung einer Bewertungssequenz.

Während in den Beispielen 104 und 105 („muss_ma REIN“ beziehungsweise „geht_s aber SCHON weiter“) das Pausenende für die Beteiligten überraschend eingeläutet wurde, wird das Ende der Pause im Gespräch zwischen Wanja und Nelli über das gerade von ihnen besuchte Theaterstücks Der Kaufmann von Venedig (siehe auch Beispiel 98) als Erleichterung von Wanja dargestellt:

$\begin{array}{ll}\text { Beispiel 107: Köln_14-06-15_Kaufmann_Gr.2 } \\ 577 \text { Nelli: } & \text { ich FAND zum beispiel die- } \\ 578 & \text { ich FAND dieses- } \\ 579 & \text { geSCHICHte mit der- } \\ 580 & (0.3) \\ 581 & \text { mit der LEIter? } \\ 582 & \text { also ich FAND das schon f- } \\ 583 & (0.2) \\ 584 & \text { UMgesetzt. } \\ 585 & \text { fand ich das schon echt GU:T. } \\ 586 & (0.6)\end{array}$


587

588

589

590

591

592

593 Wanja:

594 Nelli:

595

596 Wanja:

597

598

599

600

601

602

603

604

605

606

607

608

609

610

611

612 Nelli:

613

614 Wanja:

615 Nelli:

616

617

618

619

620

621

622

623

624

625 muss ich SAgen-

(0.8)

die:-

(6.8)

als iDEE: .

(0.2)

[ ${ }^{\circ} \mathrm{hhh}$

]

[fand icht FAND ich das scho]n:-

(2.2)

JA: aber:-

(0.6)

außer dass das irgendwie so_n SLOPstick einlage

[war- ]

Kommentar: ((Pausenklingel ertönt, ca. 8s))

Nelli: [(oh NEIN).]

Wanja: was WAR das.

ja:?

also WAS: (trickst/kriegst)-

GOTT sei dank.

es geht WEIter.

OHne uns.

((stellt Kaffeetasse ab))

Kommentar: ((Pausenklingel ertönt, ca. 12s))

Wanja: also NOCHmal.

ich WILL ja dass:-

du kannst ja [GERne noch bleiben.]

[nein nein NEIN. ]

$\operatorname{mir}$ [GEHT das-]

$[\mathrm{xxxxxx-]}$

mir GEHT es ganz ge! NAU! so.

ich MUSS da ja-

ich muss das au_nich WEItersehen.

ich weiß NICH was mich da: noch-

interessantes erWARten soll.

gut.

(0.7)

((Geschirr klappert))

dann:?

(2.3)

SORry- 
$\mathrm{Zu}$ Beginn des Gesprächsausschnitts greift Nelli einen Aspekt auf der ästhetischperformativen Ebene des Theaterstücks wieder auf, der bereits im vorherigen Gesprächsverlauf von den beiden Beteiligten bewertet wurde (siehe Beispiel 98). Während Wanja diesen Aspekt bereits negativ bewertet hat, wiederholt Nelli in Zeile 582 bis 594 ihre positive Bewertung, die sie allerdings durch Abbrüche und Disfluenzmarker sehr zögerlich realisiert. Wanja schließt an Nellis Bewertung mit partiellem Widerspruch an (Z. 596). Seine weiteren Ausführungen überlappen sich teilweise mit der Klingel, die das Ende der Pause markiert, sowie mit Nellis Interjektion „(oh NEIN)“ (Z. 600), die das Überraschungsmoment des Pausenendes für Nelli indiziert. Während Nelli unmittelbar auf das akustische Signal reagiert, fährt Wanja zunächst mit der In-Frage-Stellung der Inszenierung der „geSCHICHte mit der- mit der LEIter“ fort, bricht diese aber ab und stellt mit „GOTT sei dank. es geht WEIter. OHne uns.“ (Z. 604-606) das Ende der Pause als Entlastung dar, und impliziert somit, dass neben der Rezeption des Theaterstücks auch das Pausengespräch, in dem das Rezipierte von den beiden Beteiligten kommunikativ ver- und aufgearbeitet wird, (möglicherweise in Bezug auf die Aufnahme und das nicht mögliche vorzeitige Verlassen des Theaters) eine Belastung für ihn war. Nelli reagiert darauf nicht, sondern Wanja initiiert ab Zeile 609 eine Verständigung darüber, ob die beiden Beteiligten den Theaterbesuch vorzeitig beenden.

Auch im folgenden Beispiel ${ }^{132}$ wird das Gespräch zwischen Emma und Monika im Anschluss an das Theaterstück Brain and Beauty „von außen“, allerdings nicht durch eine Pausenklingel, sondern durch eine Ankündigung einer Theatermitarbeiterin unterbrochen, was dazu führt, dass die sich an eine Bewertung der (musikalischen) Performance einer im Stück agierenden Figur anschließende Verständigung über das Verschwinden einer anderen Figur von beiden Gesprächspartnerinnen abgebrochen wird:

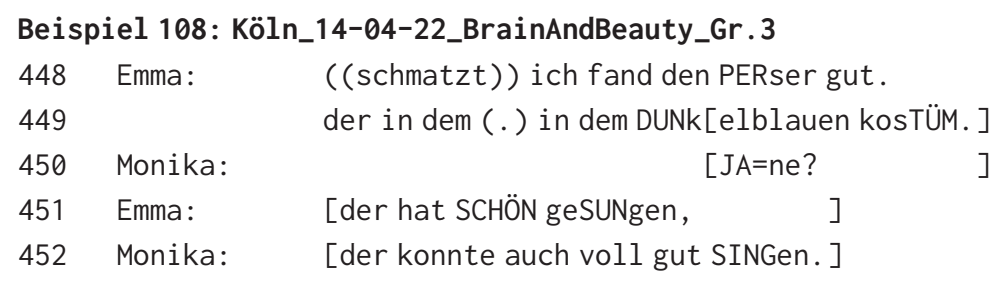

132 Siehe zu einer früheren Analyse dieses Beispiels Hrncal (2018: 288-291). 


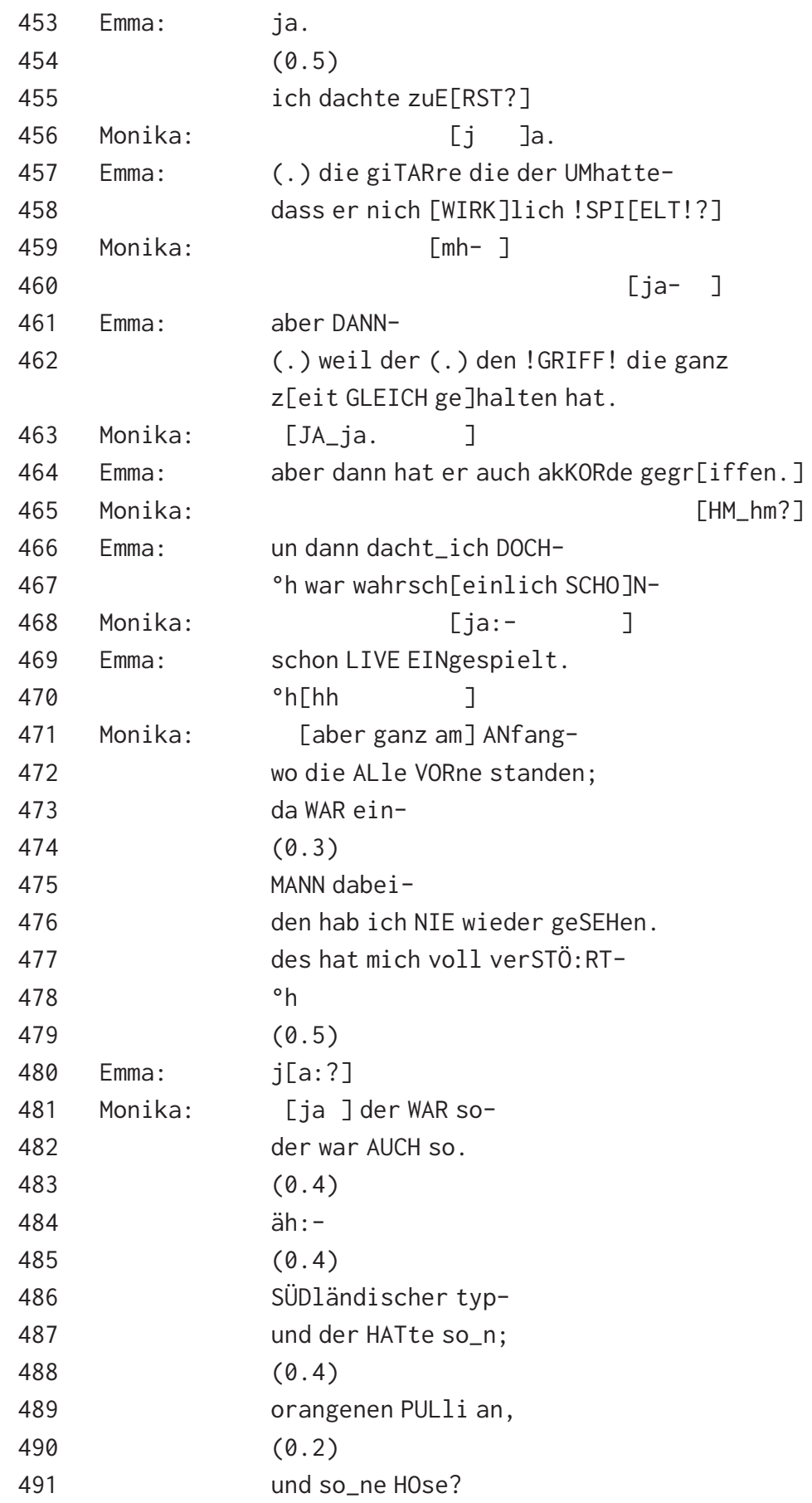




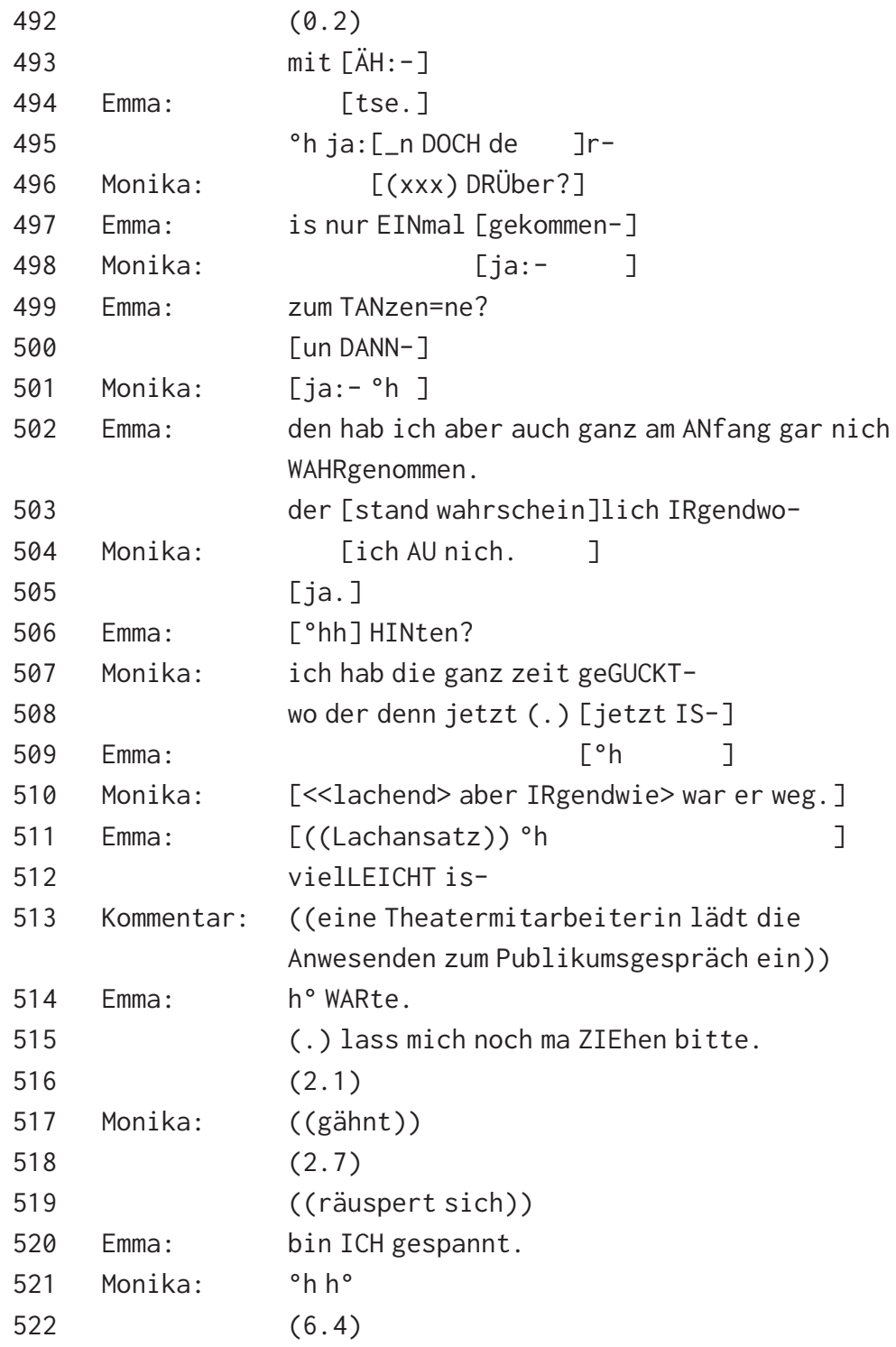

Nachdem sich die beiden Gesprächspartnerinnen ihrer Übereinstimmung hinsichtlich der Bewertung der musikalischen Performance einer Figur im Stück versichert haben (Z. 451-452), verweist Monika in Zeile 471 bis 475 auf eine Figur auf der Bühne zu Beginn des Theaterstücks (,aber ganz am ANfang- wo die ALle VORne standen; da WAR ein- (0.3) MANN dabei-“) und schildert ihre Rezeptionserfahrung (,den hab ich NIE wieder geSEHen. des hat mich voll 
verSTÖ:RT-“, Z. 476-477). Emmas steigend intoniertes „ja:?“ (Z. 480) dient Monika als Anlass zur näheren Beschreibung der Figur (Z. 481-491). Das von Emma in Zeile 495 bis 499 mit ,ja:_n DOCH der- is nur EINmal gekommen- zum TANzen =ne?“ geäußerte Identifikationsangebot bestätigt Monika in Zeile 498 und 501. Ihre in Zeile 512 eingeleitete Vermutung hinsichtlich des von ihr thematisierten Bezugsobjekts („ein MANN“) bricht Emma aufgrund der Ankündigungen einer Theatermitarbeiterin ab, die auf das Publikumsgespräch und damit vermutlich auch auf das Ende der Pause verweist (Z. 513). Im Gegensatz zu den vorangehend angeführten Beispielen wird das Pausenende von den beiden Gesprächspartnerinnen nicht kommentiert. Im weiteren Gesprächsverlauf nehmen Emma und Monika ihre Bewertungsinteraktion beziehungsweise die Verständigung darüber, was mit der von Monika angesprochenen Figur passiert ist, nicht wieder auf, sondern kehren zum Publikumsgespräch in den Theatersaal zurück.

\subsubsection{Selbstinitiierte Ausstiege aus der Bewertungsinteraktion}

In den folgenden Beispielen initiieren die Beteiligten selbst den Ausstieg aus der Bewertungsinteraktion. In den Beispielen ist $\mathrm{zu}$ beobachten, dass der Ausstieg, beispielsweise durch einen selbstinitiierten Themenwechsel, nicht abrupt erfolgen muss, sondern durch Disfluenzmarker bereits vorbereitet wird.

Beispiel $109^{133}$ entstammt einem Gespräch zwischen Anna, Annalena, Pascal und Jasmin, das im Anschluss an das Theaterstück Verrücktes Blut stattfindet (siehe auch Beispiel 64). Die vier Gesprächspartner versuchen, eingebettet in eine Bewertungsinteraktion, das Ende der von ihnen rezipierten Inszenierung zu deuten (Z. 499-514):

Beispiel 109: Siegen_14-10-26_Blut_Gr.2

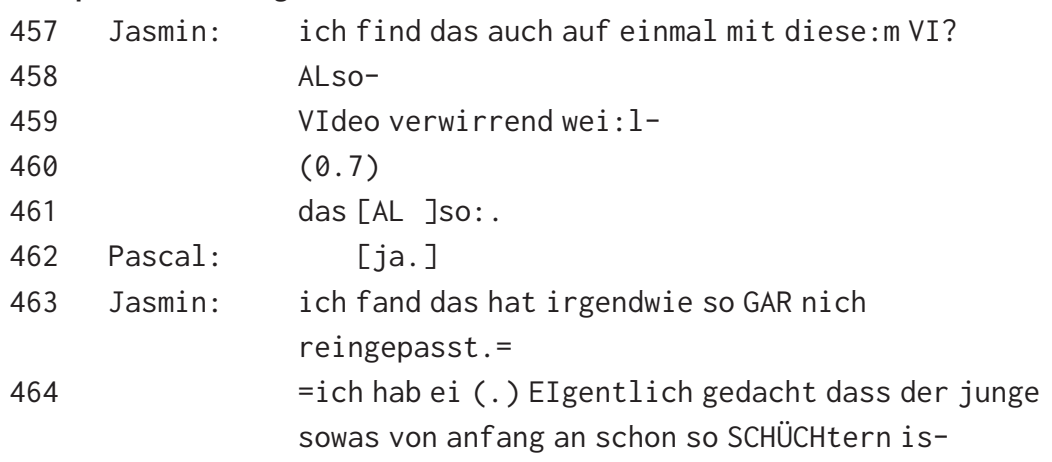

133 Siehe zu einer früheren Analyse dieses Beispiels Hrncal (2018: 293-296). 
465

466

467

468 Anna:

469 Jasmin:

470

471 Pascal:

472 Jasmin:

473

474

475

476

477

478

479

480

481

482

483

484

485

486

487

488

487

488

489

490

491

492

493

494

495

496

497

498

499

Anna: und (ah) und HALT so.

$(0.5)$

wenig SELBSTbewusstsein und sowa[s- ]

$\left[h^{\circ}\right]$

dann das VIdeo-

das fand ich irgendwie dann nochmal

verSTÖren[der.]

$$
[j a:-]=
$$

=um EHRlich zu sein-

$(0.7)$

aber das: HAben die wahrscheinlich gebrauchtum (den) EInen da zu ver!UR! teilen.

Pascal: $\quad[\mathrm{ja}-]$

Jasmin: [EIN]f[ach-]

[ja- ]

$(0.8)$

also das hätten die vielleicht anders RAUSbringen können.=

=ich fand_s jetz $\mathrm{n}$ bisschen (. ) KOmisch-

dass sie ei[nfach] nichts andres zu TUN hat als-

Jasmin: $\quad[(j a)-]$

Pascal: $\quad{ }^{\circ} \mathrm{h}$ hey. $=$

=da liegt sein HANdy.

SCHAU mal was er für !VI! [deos hat.]

Annalena:

$\left[\mathrm{hh}^{\circ}\right]$

$[j a$.

]

$[<<$ lachend $>$ ja: $>-]$

(0.2)

er HÄTte ja-

ja: .

((lacht))

(fast) dann vielleicht einfach SELBST die-

WAFfe !KRIE! gen !KÖN!nen.

und das dann SELBST ! AN! sprechen können als-

Anna: ja-

$(0.7)$

[ja. ]

$\left[{ }^{\circ} \mathrm{h}\right] \mathrm{ja}$ und (.) geNAU.

am ende HATte er ja dann irgendwie ne ALso-

${ }^{\circ} \mathrm{h}$ [am ENde hab $\mathrm{i}$ ]ch das !ECHT! nich verstan[den.] 


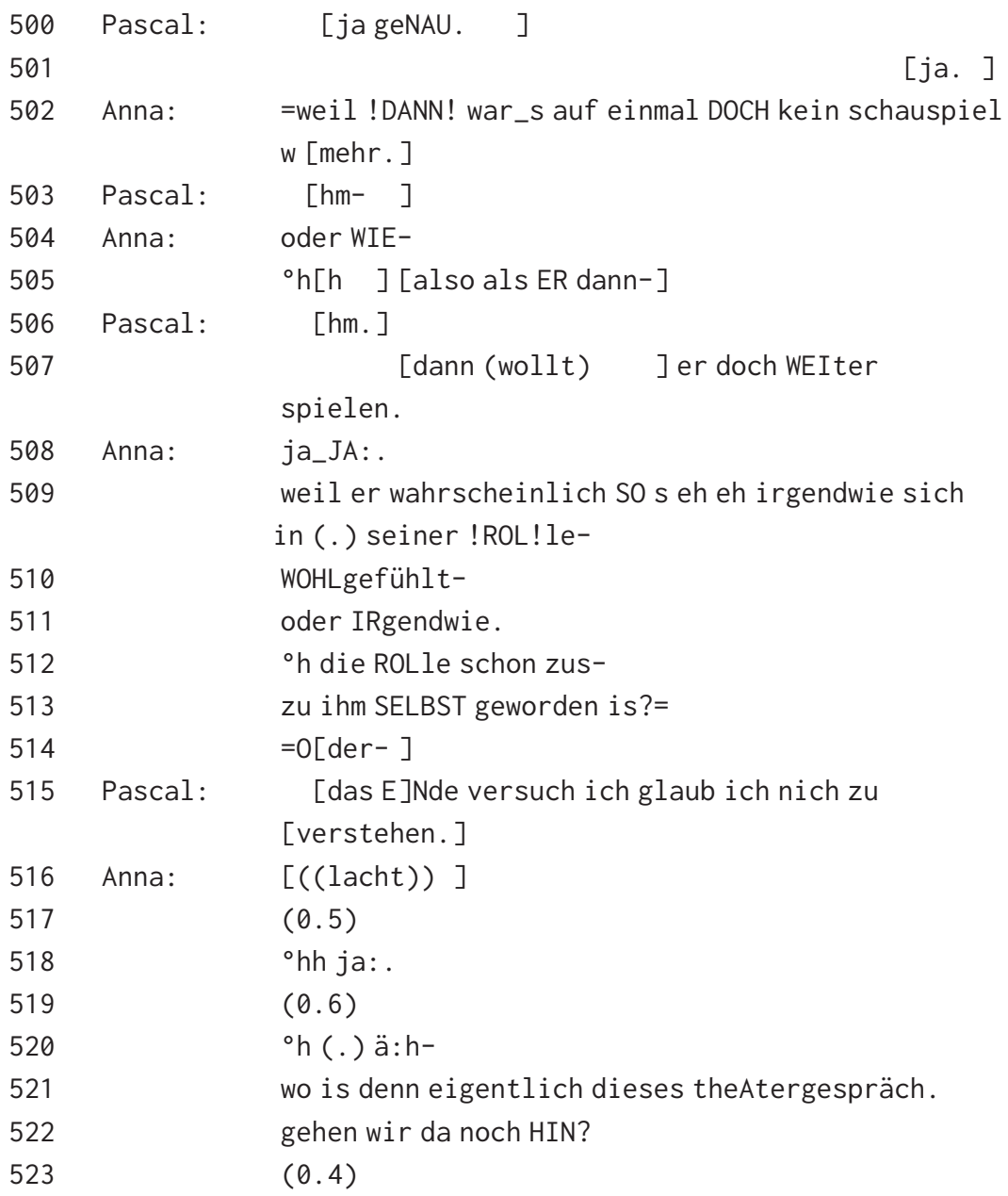

$\mathrm{Zu}$ Beginn des Ausschnitts greift Jasmin eine Stelle im Theaterstück heraus (Z. 457) und schildert ihr inneres Erleben (Z. 459). Im Anschluss begründet sie ihre Wahrnehmung vage damit, dass die Stelle, auf die sie verweist, ,irgendwie so GAR nich reingepasst.“ hat (Z. 463). Ab Zeile 474 versuchen die Beteiligten, die Gründe für die Präsentation eines Videos im Stück nachzuvollziehen. Die Suche nach Gründen führt Pascal zu einem alternativen Inszenierungsvorschlag (Z. 480 und 488-493). Anna verweist in Zeile 499 auf das für sie nicht nachvollziehbare Ende des Theaterstücks, woraufhin sich Pascal weiteren Deutungsversuchen des am Ende des Theaterstücks Präsentierten verweigert (Z. 515). Anna quittiert diese Positionierung von Pascal mit einem Lachen (Z. 516) und initiiert keine weitere Expansion der Deutungssequenz. Eine endgültige 
Deutung bleibt offen. Nach einer kurzen Pause, einem gedehnten „ja:“ in Zeile 518 und weiteren Disfluenzmarkern initiiert Anna mit den Fragen „wo is denn eigentlich dieses theAtergespräch. gehen wir da noch HIN?“ eine Verständigung über das weitere Vorgehen im Rahmen des gemeinsamen Theaterbesuchs. Nach diesem Themenwechsel greift keiner der Beteiligten das zuvor bearbeitete Thema nochmals auf.

Durch einen Themenwechsel auf die situativen Umstände des Pausengesprächs erfolgt auch im folgenden Gespräch zwischen Susanne und Gudrun in der Pause des Theaterstücks Der Kaufmann von Venedig der Ausstieg aus der Bewertungsinteraktion:

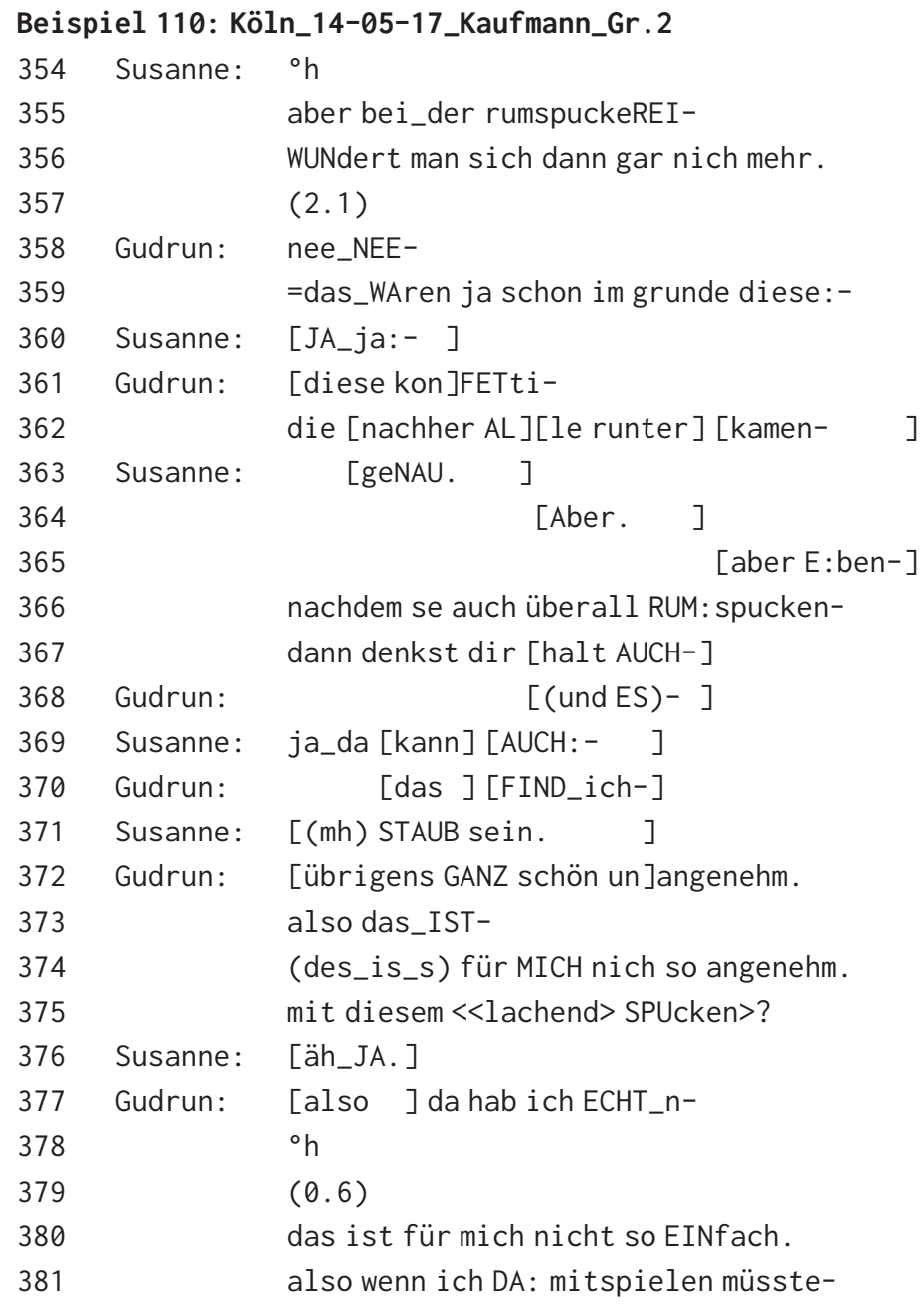


382

383

384

385

386

387

388

389

390

391

392

393

394

395

396

397

398

399

400

401

402

403

404

405

406

407

408

409

410

411

412

413

414

415

416

417 hätt_ich da ein echtes proBLEM mit-

Susanne: aber_der hat m[ir toTAL gu ]t gefallen-

Gudrun:

[KRIEgen wir-]

Susanne:

der_sp (.) der FIND_ich.

der JUde-

der spielt WAHNsinnig toll-

Gudrun: der am MEISten spuckt.

$(($ lacht $))(($ lacht $))$

Susanne: ja:-

aber ich FIND der:-

Gudrun: JA xxx-

Susanne: (der_is) KLASse.

(2.2)

Gudrun: ja das [kannst_(mir) auch am MEISten en]twickeln-

Susanne: [ganz GROSSartig-

Gudrun: diese RoLle.

$=$ ne?

die (. ) hat im GRUNde-

auch die_mei (.) das meiste potenziALvon der ROLle her.

(. ) wobei das ja nicht die HAUPTfigur ist.

von SHAKEspeare aus-

ist [es eigent]lich der KAUFmann eher.

Susanne: [NEE_e? ]

(1.6)

stimmt.

(1.2)

Gudrun: HM_hm,

wie lang ist jetzt die PAUse?

(0.3)

Susanne: keine AHnung-

(0.7)

LASS uns_doch nochmal [da (hinten)-

]

[(ich) würd nämlich sch]on

nochmal auf die toiLETte-

$(0.4)$

In Zeile 355 nimmt Susanne mit der „rumspuckeREI“ Bezug auf einen Aspekt auf der ästhetisch-performativen Ebene des Theaterstücks, den Gudrun in Zeile 372 
implizit negativ bewertet. Mit ihrer positiven Bewertung ,aber_der hat mir toTAL gut gefallen“ (Z. 383) fokussiert Susanne die im Theaterstück agierende beziehungsweise spuckende Figur, distanziert sich von Gudruns Position (,aber“) und schreibt ihrer Äußerung damit den Status einer negativen Bewertung zu. In den folgenden Zeilen bewertet Susanne die schauspielerische Leistung (Z. 387) und den Schauspieler oder die von ihm verkörperte Figur (Z. 393 und 396) nochmals explizit positiv. Gudrun verweist daraufhin auf das Entwicklungspotenzial der Rolle und klärt Susanne darüber auf, dass „von SHAKEspeare aus“ (Z. 403) der Kaufmann die Hauptfigur des Stücks darstellt. Obwohl Susanne mit ihrer Nachfrage „NEE_e?“ einen Status des Nichtwissens indiziert, stimmt sie nach einer kurzen Pause Gudrun zu (Z. 407). Gudrun quittiert dies nach einer Pause durch ein leicht steigend intoniertes „HM_hm“ (Z. 409) und wechselt nach einer weiteren Pause mit der Frage nach der Länge der Theaterpause das Thema.

Ein ähnlicher Ausstieg aus der Bewertungsinteraktion ist im Gespräch ${ }^{134}$ zwischen Kristina, Hildegard und Irmgard im Anschluss an das Theaterstück Habe die Ehre zu beobachten. Nachdem die Gesprächspartnerinnen das Bühnenbild bewertet und damit verbundene Klischees ironisierend zum Thema gemacht haben, fragt Kristina in Zeile 368 nach dem Namen eines Schauspielers:

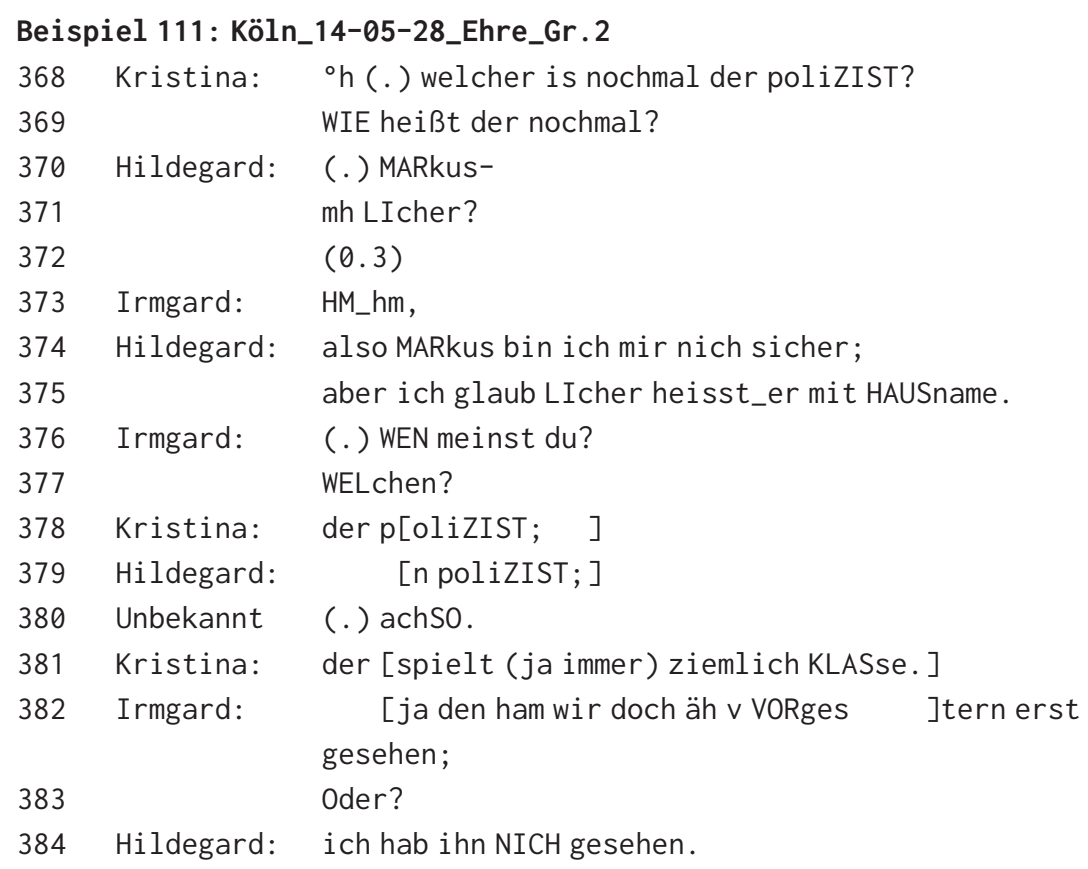

134 Siehe zu einer früheren Analyse dieses Beispiels Hrncal (2018: 291-293). 
385

386

387

388

389

390

391

392

393

394

395

396

397

398

399

400

401

402

403

404

405

406

407

408

(2.5)

Kristina: [HM_hm, ]

Irmgard: [war der nich] in dem STÜCK-

(der) KAUFmann von veNEdig dabei?

Hildegard: ich glaube NICH;

Kristina: ne:-

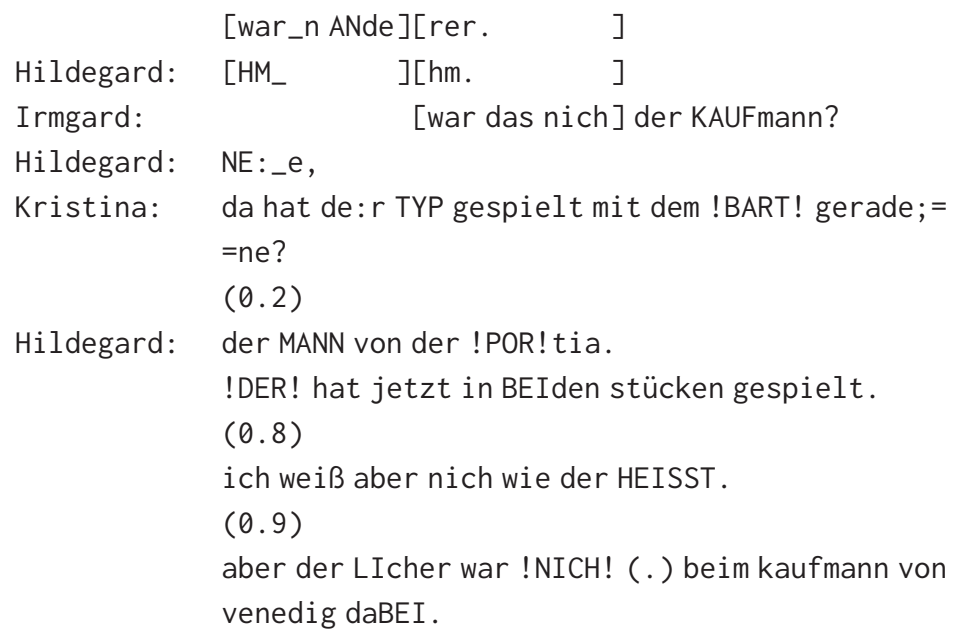

Nachdem Hildegard hinsichtlich des Namens des Schauspielers ein als Vermutung gerahmtes Identifizierungsangebot verbalisiert hat (,ich glaub LIcher heisst_er“, Z. 375), folgt eine explizit positive Bewertung seiner schauspielerischen Leistung auf der ästhetisch-performativen Ebene des Stücks durch Kristina (,der spielt (ja immer) ziemlich KLASse.“, Z. 381). Dem von Irmgard in Überlappung mit Kristinas Bewertung geäußerten Verweis ,ja den ham wir doch äh v VORgestern erst gesehen; Oder?“ (Z. 382-383) widersprechen Hildegard in abgeschwächter und Kristina in direkter Art und Weise (Z. 384-391), was eine interaktive Aushandlung der Sprecherinnen nach sich zieht, welcher Schauspieler in welchem Stück gespielt hat. Hildegards Erläuterungen in Zeile 398 bis 403 werden von Irmgard nach einer kurzen Pause in Zeile 405 mit einem leicht steigend intonierten „HM_hm“ quittiert. Die sich daran anschließende Pause (Z. 406) sowie das von Irmgard geäußerte gedehnte ,ja:;“ (Z. 407) bereiten den Ausstieg aus der aktuell bearbeiteten Sequenz vor, der mit dem von Irm- 
gard mittels ihrer Frage „was für_n restauRANT suchen wa denn (aus),“ (Z. 408) initiierten Themenwechsel auf ein Small Talk-Thema (siehe Kapitel 4.1) im weiteren Sinne vollzogen und als abgeschlossen behandelt wird. Weder die Bewertung Kristinas noch die Verständigung über den Schauspieler werden nach Irmgards Themenwechsel nochmals aufgegriffen. Stattdessen verweist Hildegard auf eine im Theaterfoyer anwesende Person, die dann für eine kurze Zeit in den Fokus des Gesprächs rückt, bis Irmgard wieder den geplanten Restaurantbesuch thematisiert. ${ }^{135}$

\subsubsection{Ergebnisse}

Die in Kapitel 8.4 analysierten Beispiele haben gezeigt, dass die Beteiligten das akustische Signal, dass in der Regel das Pausenende markiert, zum Teil in ihren Gesprächen aufgreifen und einen Abbruch der Bewertungs- beziehungsweise sich daran anschließenden Deutungssequenz relevant setzen und vollziehen sowie ihr Gegenüber zu einem Abbruch seiner Äußerungen verpflichten können.

\section{Indizieren semi-institutioneller Restriktionen}

Mit der Art und Weise des Verweisens auf das Pausenende unmittelbar nach dem Ertönen des akustischen Signals indizieren die Beteiligten gleichzeitig ihre Überraschtheit (,is das schon WEItergegangen?“ und ,jetz geht_s aber SCHON weiter.“, Beispiel 87, Kapitel 8.4.1) und verweisen damit implizit auf die Tatsache, dass das Pausenende oftmals nicht exakt vorhersehbar ist (vgl. auch Habscheid/Linz 2018). Dass die aktuelle Themenbearbeitung durch eine Störung von außen (in diesem Fall die Ankündigung einer Mitarbeiterin des Theaters) auch abgebrochen werden kann, ohne dies explizit zu thematisieren, zeigt Beispiel 108 aus dem Gespräch zwischen Emma und Monika. Die bereits in Kapitel 2.3.2 angesprochenen semi-institutionellen Restriktionen manifestieren sich in den vorliegenden Gesprächen besonders hinsichtlich des in Kapitel 8.4 in den Blick genommenen Zeitpunkts der Gesprächsbeendigung, der zum Beispiel in Ausschnitt 104 (Kapitel 8.4.1) durch das in der Äußerung von Susanne „glaub ich muss_ma REIN.“ enthaltene Modalverb müssen von den Beteiligten selbst als Restriktion relevant gesetzt wird.

135 Mit dem Ausstieg aus der vorliegenden Sequenz endet in diesem Gespräch auch der kommunikative Austausch über das Theaterstück, in dessen Anschluss nur noch private Themen (Auswahl eines Restaurants, Urlaubspläne usw.) bearbeitet werden. 


\section{Rückzug aus erhöhter gemeinsamer Beteiligung}

Selbstinitiierte Ausstiege aus der Bewertungsinteraktion werden in den in Kapitel 8.4.2 angeführten Beispielen dann vorgenommen, wenn die Beteiligten in ihren Bewertungen und Deutungen übereinstimmen und die Bewertungssequenz von ihnen als abgeschlossen behandelt sowie ein Themenwechsel (unter anderem mit Bezug auf private Aspekte oder auf das aktuelle Setting) vollzogen wird. Dieser Form des selbstinitiierten Ausstiegs gehen in den angeführten Beispielen Disfluenzmarker wie beispielsweise Pausen oder Wortdehnungen voran. Die in Kapitel 8.4.2 angeführten selbstinitiierten Ausstiege aus der Bewertungsinteraktion umfassen somit die bereits in Kapitel 2.3.1 mit Verweis auf Goodwin und Goodwin (1992) sowie auch bei Goodwin (2018) angesprochenen Verfahren der Interagierenden, um sich von einer erhöhten gemeinsamen Beteiligung zurückzuziehen. Dies ist bei den Beispielen, die im Rahmen der Betrachtung von außen initiierter Ausstiege in Kapitel 8.4.1 angeführt wurden, nicht der Fall. Da der Rückzug von allen Beteiligten gleichzeitig vollzogen wird, werden - anders als bei den Fällen des Rückzugs einzelner Sprecher aus Bewertungsinteraktionen aufgrund von Wissensasymmetrien (siehe Kapitel 8.3) - keine Maßnahmen getroffen, um eine Reintegration dieser Sprecher in die Interaktion zu begünstigen.

Ausstiege aus der Bewertungsinteraktion fallen nicht zwangsweise auch mit dem Ende des gesamten Gesprächs zusammen, sondern können zu jeglichem Zeitpunkt vorgenommen werden. Das bedeutet nicht zwangsläufig, dass nach dem Ausstieg aus der Bewertungsinteraktion diese im weiteren Gesprächsverlauf nicht noch einmal aufgegriffen wird, sondern dass diese zu einem späteren Zeitpunkt im Gespräch, beispielsweise nach der Getränkebestellung oder einer side sequence (vgl. Jefferson 1972), in der private Themen bearbeitet werden, von einem der Beteiligten wieder relevant gesetzt werden kann.

Die in diesem Kapitel angeführten Ausstiege aus Bewertungsinteraktionen zeigen zudem, dass Ausstiege - unabhängig davon, ob sie durch ein Signal von außen relevant gesetzt oder selbstinitiativ initiiert werden, von den Beteiligten kooperativ vollzogen werden. 
